\title{
Cardiovascular magnetic resonance : a key to imaging cardiac function
}

Citation for published version (APA):

Schalla, S. M. (2015). Cardiovascular magnetic resonance : a key to imaging cardiac function. [Doctoral Thesis, Maastricht University]. Datawyse / Universitaire Pers Maastricht. https://doi.org/10.26481/dis.20150706ss

Document status and date:

Published: 01/01/2015

DOI:

10.26481/dis.20150706ss

Document Version:

Publisher's PDF, also known as Version of record

\section{Please check the document version of this publication:}

- A submitted manuscript is the version of the article upon submission and before peer-review. There can be important differences between the submitted version and the official published version of record.

People interested in the research are advised to contact the author for the final version of the publication, or visit the DOI to the publisher's website.

- The final author version and the galley proof are versions of the publication after peer review.

- The final published version features the final layout of the paper including the volume, issue and page numbers.

Link to publication

\footnotetext{
General rights rights.

- You may freely distribute the URL identifying the publication in the public portal. please follow below link for the End User Agreement:

www.umlib.nl/taverne-license

Take down policy

If you believe that this document breaches copyright please contact us at:

repository@maastrichtuniversity.nl

providing details and we will investigate your claim.
}

Copyright and moral rights for the publications made accessible in the public portal are retained by the authors and/or other copyright owners and it is a condition of accessing publications that users recognise and abide by the legal requirements associated with these

- Users may download and print one copy of any publication from the public portal for the purpose of private study or research.

- You may not further distribute the material or use it for any profit-making activity or commercial gain

If the publication is distributed under the terms of Article $25 \mathrm{fa}$ of the Dutch Copyright Act, indicated by the "Taverne" license above, 


\section{GARDIOVASCULAR \\ MAGNETC RESONANCE A key to imaging cardiac function}

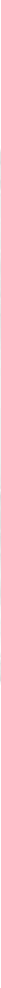


(C) S. Schalla, Maastricht 2015

ISBN 9789461594334

Cover illustration by S. Schalla

Printed by Datawyse / Universitaire Pers Maastricht 


\title{
Cardiovascular magnetic resonance A key to imaging cardiac function
}

\author{
PROEFSCHRIFT \\ ter verkrijging van de graad van doctor \\ aan de Universiteit Maastricht, \\ op gezag van de Rector Magnificus, Prof. dr. L.L.G. Soete, \\ volgens het besluit van het College van Decanen, \\ in het openbaar te verdedigen \\ op maandag 6 juli 2015 om 10:00 uur
}

door

Simon Michael Schalla

geboren op 24 mei 1967 te Kiel

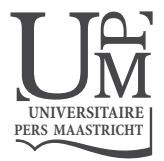




\section{Promotores}

Prof. dr. H.J.G.M. Crijns

Prof. dr. J.E. Wildberger

Beoordelingscommissie

Prof. dr. F.W. Prinzen (Chair)

Prof. dr. A. Bücker

Prof. dr. T. Delhaas

Dr. E. Kooi

Prof. dr. F. Rademakers

Prof. dr. A.C. van Rossum 


\section{Contents}

Chapter 1 Introduction 7

Part I - Real-time imaging- interventional MR 13

Chapter 2 Magnetic resonance-guided cardiac catheterization in a swine model 15 of atrial septal defect

Chapter 3 Balloon sizing and transcatheter closure of acute atrial septal defects 29 guided by magnetic resonance fluoroscopy: assessment and validation in a large animal model

Chapter 4 Magnetic resonance flow measurements in real-time: comparison with a standard gradient-echo technique

Chapter 5 Real-time MR image acquisition during high-dose dobutamine hydrochloride stress for detecting left ventricular wall-motion abnormalities in patients with coronary arterial disease

Part II - Ischemic heart disease

Chapter 6 Accentuation of high susceptibility of hypertrophied myocardium to ischemia: complementary assessment of Gadophrin-enhancement and left ventricular function with MRI

Chapter 7 Effect of potassium-channel opener therapy on reperfused infarction in hypertrophied hearts: demonstration of preconditioning by using functional and contrast-enhanced magnetic resonance imaging

Chapter 8 Long-term oral treatment with nicorandil prevents the progression of left ventricular hypertrophy and preserves viability

Chapter 9 Incremental value of cardiovascular magnetic resonance over echocardiography in the detection of acute and chronic myocardial infarction

Chapter 10 Non-invasive assessment of microvascular dysfunction in patients with cardiac syndrome $X$

Part III - Nonischemic heart disease

Chapter 11 Replacement and reactive myocardial fibrosis in idiopathic dilated cardiomyopathy: comparison of magnetic resonance imaging with right ventricular biopsy 
Chapter 12 Right ventricular function in dilated cardiomyopathy and ischemic heart disease: assessment with non-invasive imaging

Chapter 13 Structural and functional cardiac changes in myotonic dystrophy type 191 1: a cardiovascular magnetic resonance study

Part IV 205

Chapter 14 Summary, discussion and future perspectives

Nederlandse samenvatting

Valorization

Dankwoord 231

Curriculum vitae 235

List of publications 239 
1

Introduction 



\section{Introduction}

"Sooner or later all interesting cardiac patients will get an MR scan." This often quoted statement is no longer true because of two reasons. First, all patients are interesting. Even most common diseases are associated with a huge variety of different complaints, signs and symptoms. This is one of the main reasons why cardiac imaging needs to be performed so frequently in addition to taking the medical history and carrying out the physical examination. Second, cardiovascular magnetic resonance imaging (CMR) is currently used on a routine basis for many patients with heart disease and not only for niche indications. Since the first publication of a cross sectional magnetic resonance image of a human chest in 1977, ${ }^{1}$ CMR has emerged as a reliable imaging technique and serves as a reference standard for regional wall motion, global and regional systolic function, myocardial mass and hypertrophy, fibrosis and infarct imaging, viability as well as anatomic imaging of e.g. the pericardium, aorta and congenital heart disease. ${ }^{2-6}$ In addition, the non-invasive detection of ischemia with CMR has a high diagnostic accura$\mathrm{cy}^{7-14} \mathrm{CMR}$ is less well established for imaging of the coronary arteries, diastolic function and valvular disease except for aortic and pulmonary valve regurgitation. Several unique techniques such as 3D flow measurement, T1 and T2 mapping, feature tracking and perfusion imaging without the use of contrast media have recently been added to scan protocols or will be introduced into clinical routine in the future. ${ }^{15-18}$

Current CMR scan techniques include ECG-gated cine balanced steady state free precession for imaging function, black blood and also balanced steady state free precession sequences for anatomy, late gadolinium enhancement inversion recovery sequences for infarct or fibrosis, and saturation recovery sequences for contrast agent first-pass myocardial perfusion. There are many more clinical and experimental sequences and imaging techniques available for imaging of edema, T1 and T2-mapping, T2* analysis, angiography with and without the use of contrast material, tagging and spectroscopy. Cardiac magnetic resonance imaging started in the $1970^{\text {ties }}$ with spectroscopy for assessing myocardial phosphate metabolism in isolated rat hearts. ${ }^{19}$ The first publication of a cross section of the human chest came from Damadian et al. ${ }^{1}$ It is not known when the first MR scans to specifically image the heart were performed since the heart could be recognized on those early cross section chest images. ${ }^{20}$ Hawkes et al. probably published the first study specifically directed at cardiac MR imaging in $1981 .^{21}$ At least the authors were excited about the quality of the images, but recognized cardiac and respiratory motion as limiting factors and proposed the use of ECG-triggering to improve image quality. In 1984, Higgins et al. were the first to publish cardiac images with good anatomical details using ECG-gating. ${ }^{22}$ ECG-synchronization resulted in superior image quality compared to the other cardiac gating signal methods from peripheral pulse and Doppler flow signals. Since then, many more improvements have been achieved. Sometimes it was the reinvention of the already known. Steady state free 
precession sequences were known for many years, but only after advances in gradient hardware had been made they could be introduced into daily practice. ${ }^{20,23}$

Data from the United States show that more than 21 million Americans suffered in 2010 from cardiac disease. There were more than 700000 deaths due to cardiovascular disease including stroke and hypertension. ${ }^{24}$ The burden of cardiac disease in the Netherlands in 2012 was 20555 cardiac deaths and 202945 hospital admissions. ${ }^{25}$ The hospital admissions consisted of 83941 patients with myocardial ischemia, 3009 congenital heart disease, 9612 valve disease, 2948 cardiac infections, and 103435 other cardiac causes including heart failure. In total more than 1 million patients suffer from cardiovascular disease in the Netherlands. In a conservative estimation approximately at least half of the patients admitted will undergo cardiac imaging during or shortly after admission during the following outpatient visit. Thus, at least more than 100000 cardiac imaging studies might annually be performed for hospital admissions. The number of cardiac imaging studies for outpatient clinic visits is not known.

This thesis describes the different aspects of MR imaging of ventricular dysfunction. CMR is currently the only comprehensive cardiac imaging method to assess cardiac morphology, global and regional LV and RV-function, mass, perfusion and viability, fibrosis and scar characteristics, coronary artery anatomy and the thoracic vasculature. It is considered reliable and highly reproducible $e^{4,26}$ and it does not use ionizing radiation. Intravenous MR contrast material is generally safe if not used in patients with end stage kidney disease. Thus, the aim of this thesis was to demonstrate the value of CMR in assessing cardiac dysfunction. Therefore, the thesis is divided into three parts.

The first part of the thesis focusses on fast, real-time MR imaging which is important for scan planning, rapid interpretation already during scanning, and catheter guidance for interventional MR. In chapters 2 and 3, left and right heart catheterization with real-time MR catheter guidance (MR fluoroscopy) in atrial septal defect (ASD) is presented, small shunt volumes detected, balloon sizing and closure of ASDs performed. A real-time flow measurement technique is presented in Chapter 4 . Chapter 5 is a transition to the second part of the thesis. In this chapter a real-time imaging technique is described and evaluated during dobutamine stress in patients with coronary artery disease.

In the second part of the thesis MR imaging of ischemic heart disease is described. Chapters 6 to 8 are focussed on measuring infarct size in acute myocardial infarction of the hypertrophied heart with pre- and postconditioning. Chapter 9 compares CMR with echocardiography to detect acute and chronic myocardial infarct. In chapter 10 research on myocardial ischemia due to small vessel disease is presented.

In the third part an overview of different aspects of MR imaging of non-ischemic heart disease is given. Chapter 11 assesses focal and interstitial myocardial fibrosis in dilated cardiomyopathy and Chapter 12 the right ventricular function in dilated cardiomyopathy and ischemic heart disease. Chapter 13 is about cardiac findings in patients with myotonic dystrophy. 
The general discussion provides a critical appraisal of the current status and future developments of cardiac MR imaging.

\section{References}

1. Damadian R, Goldsmith M, Minkoff L. NMR in cancer: XVI. FONAR image of the live human body. Physiol Chem Phys 1977;1977;9:97-100.

2. Bottini PB, Carr AA, Prisant LM, et al. Magnetic resonance imaging compared to echocardiography to assess left ventricular mass in the hypertensive patient. Am J Hypertens. 1995;8:221-228.

3. Kim RJ, Wu E, Rafael A, et al. The use of contrast-enhanced magnetic resonance imaging to identify reversible myocardial dysfunction. N Engl J Med. 2000;343:1445-1453.

4. Bellenger NG, Davies LC, Francis JM, et al. Reduction in sample size for studies of remodeling in heart failure by the use of cardiovascular magnetic resonance. J Cardiovasc Magn Reson 2000;2:271-278.

5. Assomull RG, Prasad SK, Lyne J, et al. Cardiovascular magnetic resonance, fibrosis, and prognosis in dilated cardiomyopathy. J Am Coll Cardiol. 2006;48:1977-1985.

6. Heathfield E, Hussain T, Qureshi S, et al. Cardiovascular magnetic resonance imaging in congenital heart disease as an alternative to diagnostic invasive cardiac catheterization: a single center experience. Congenit Heart Dis. 2013;8:322-327.

7. Paetsch I, Jahnke C, Wahl A, et al. Comparison of dobutamine stress magnetic resonance, adenosine stress magnetic resonance, and adenosine stress magnetic resonance perfusion. Circulation. 2004;110:835-842.

8. Nagel E, Lehmkuhl HB, Bocksch W, et al. Noninvasive diagnosis of ischemia-induced wall motion abnormalities with the use of high-dose dobutamine stress MRI: comparison with dobutamine stress echocardiography. Circulation. 1999;99:763-770.

9. Kamiya K, Sakakibara M, Asakawa N, et al. Cardiac Magnetic Resonance Performs Better in the Detection of Functionally Significant Coronary Artery Stenosis Compared to Single-Photon Emission Computed Tomography and Dobutamine Stress Echocardiography. Circ J 2014;11:11.

10. Heitner JF, Klem I, Rasheed D, et al. Stress cardiac MR imaging compared with stress echocardiography in the early evaluation of patients who present to the emergency department with intermediate-risk chest pain. Radiology. 2014;271:56-64.

11. Desai RR, Jha S. Diagnostic performance of cardiac stress perfusion MRI in the detection of coronary artery disease using fractional flow reserve as the reference standard: a meta-analysis. AJR Am J Roentgenol. 2013;201:W245-252.

12. Jaarsma C, Leiner T, Bekkers SC, et al. Diagnostic performance of noninvasive myocardial perfusion imaging using single-photon emission computed tomography, cardiac magnetic resonance, and positron emission tomography imaging for the detection of obstructive coronary artery disease: a meta-analysis. J Am Coll Cardiol. 2012;59:1719-1728.

13. Hendel RC, Patel MR, Kramer CM, et al. ACCF/ACR/SCCT/SCMR/ASNC/NASCI/SCAI/SIR 2006 appropriateness criteria for cardiac computed tomography and cardiac magnetic resonance imaging: a report of the American College of Cardiology Foundation Quality Strategic Directions Committee Appropriateness Criteria Working Group, American College of Radiology, Society of Cardiovascular Computed Tomography, Society for Cardiovascular Magnetic Resonance, American Society of Nuclear Cardiology, North American Society for Cardiac Imaging, Society for Cardiovascular Angiography and Interventions, and Society of Interventional Radiology. J Am Coll Cardiol. 2006;48:1475-1497.

14. Hundley WG, Bluemke DA, Finn JP, et al. ACCF/ACR/AHA/NASCI/SCMR 2010 expert consensus document on cardiovascular magnetic resonance: a report of the American College of Cardiology Foundation Task Force on Expert Consensus Documents. J Am Coll Cardiol. 2010;55:2614-2662. 
15. Taylor RJ, Umar F, Moody WE, et al. Feature-tracking cardiovascular magnetic resonance as a novel technique for the assessment of mechanical dyssynchrony. Int J Cardiol. 2014;175:120-125.

16. Moon JC, Messroghli DR, Kellman P, et al. Myocardial T1 mapping and extracellular volume quantification: a Society for Cardiovascular Magnetic Resonance (SCMR) and CMR Working Group of the European Society of Cardiology consensus statement. J Cardiovasc Magn Reson. 2013;15:92.

17. von Knobelsdorff-Brenkenhoff F, Trauzeddel RF, Barker AJ, et al. Blood flow characteristics in the ascending aorta after aortic valve replacement--a pilot study using 4D-flow MRI. Int J Cardiol. 2014;170:426-433.

18. Walcher T, Manzke R, Hombach V, et al. Myocardial perfusion reserve assessed by T2-prepared steadystate free precession blood oxygen level-dependent magnetic resonance imaging in comparison to fractional flow reserve. Circ Cardiovasc Imaging. 2012;5:580-586.

19. Gadian DG, Hoult DI, Radda GK, et al. Phosphorus nuclear magnetic resonance studies on normoxic and ischemic cardiac tissue. Proc Natl Acad Sci U S A. 1976;73:4446-4448.

20. Geva T. Magnetic resonance imaging: Historical perspective. J Cardiovas Magn Reson. 2006;8:573-580.

21. Hawkes RC, Holland GN, Moore WS, et al. Nuclear magnetic resonance (NMR) tomography of the normal heart. J Comput Assist Tomogr. 1981;5:605-612.

22. Lanzer P, Botvinick EH, Schiller NB, et al. Cardiac imaging using gated magnetic resonance. Radiology. 1984;150:121-127.

23. Jolesz FA, Patz S, Hawkes RC, et al. Mapping of normal and abnormal cerebrospinal fluid flow/motion patterns using steady state free precession imaging. Acta Radiol Suppl 1986;369:302-304.

24. Go AS, Mozaffarian D, Roger VL, et al. Heart disease and stroke statistics--2014 update: a report from the American Heart Association. Circulation. 2014;129:e28-e292.

25. Vaartjes I KC, van Dis I, Visseren FLJ, Bots ML. . Hart- en vaatziekten in Nederland 2013, cijfers over leefstijl, risicofactoren, ziekte en sterfte. Den Haag: Hartstichting, 20132013.

26. Grothues F, Smith GC, Moon JC, et al. Comparison of interstudy reproducibility of cardiovascular magnetic resonance with two-dimensional echocardiography in normal subjects and in patients with heart failure or left ventricular hypertrophy. Am J Cardiol. 2002;90:29-34. 

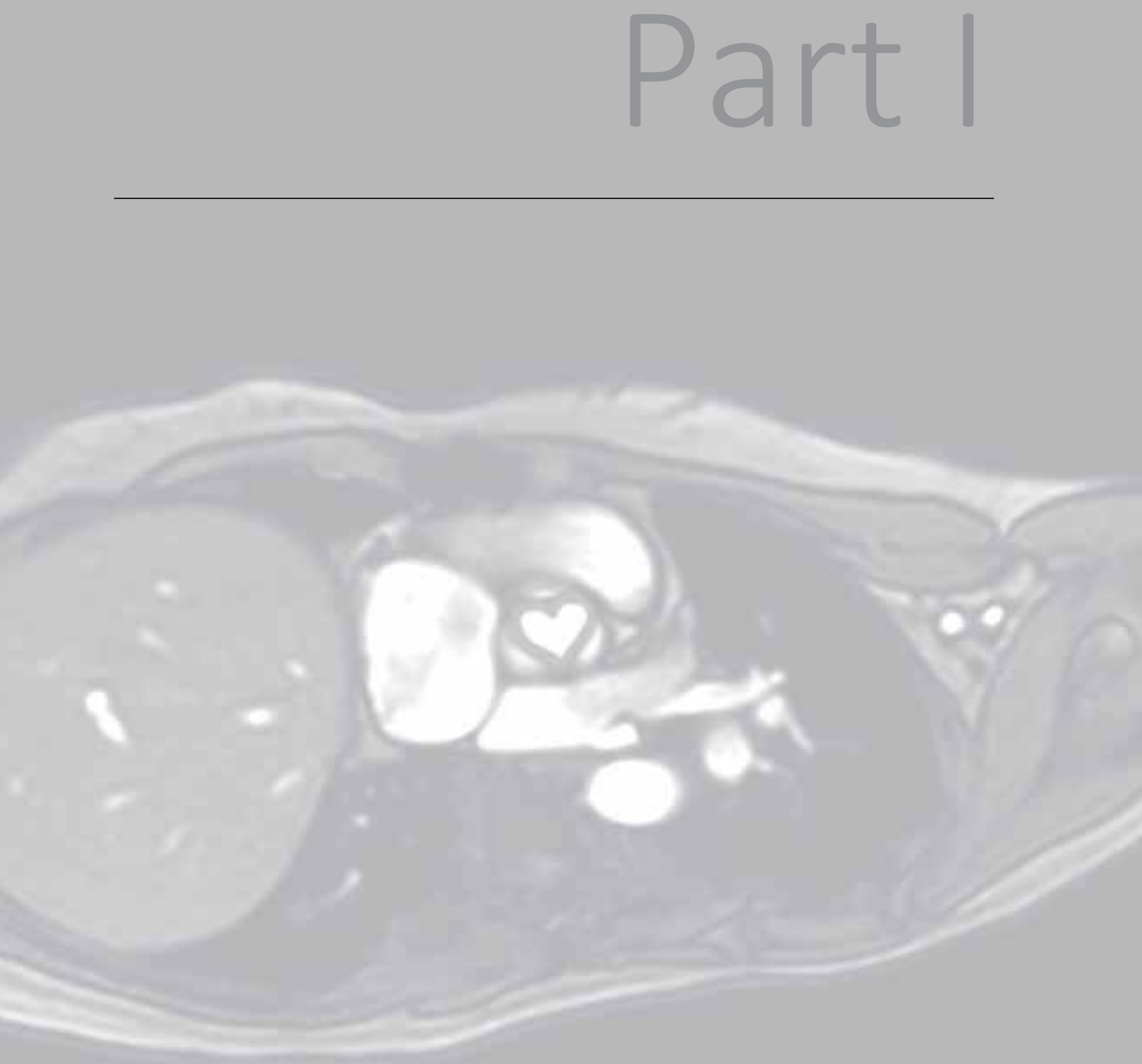



\section{Magnetic resonance-guided cardiac catheterization in a swine model of atrial septal defect}

S. Schalla, M. Saeed, C.B. Higgins, A. Martin, O, Weber, P. Moore.

Circulation. 2003 Oct 14;108(15):1865-70. 


\section{Abstract}

Background: Radiation exposure during cardiac catheterization, limited image planes, and poor soft tissue definition are disadvantages of x-ray fluoroscopy that could be overcome with the use of MRI. This study evaluates the feasibility of real-time MRI (MR fluoroscopy) to guide left and right heart catheterization.

Methods and results: Anesthetized pigs $(n=7)$ with defects of the atrial septum were catheterized using venous and arterial access. A prototype active tracking catheter was used to obtain blood pressures and samples from cardiac chambers and great vessels using antegrade, transseptal, and retrograde approaches. MR fluoroscopy was used for catheter steering. Velocity-encoded cine MRI was used to measure pulmonary and aortic blood flow to calculate vascular resistances. Image planes used during catheter manipulation used rapid sequencing to planes directed by the operator to include the tip of the catheter and the chamber to be entered. All areas of interest were effectively entered, and samples were obtained. In the presence of an acute atrial septal defect, a Qp/Qs ratio of $1.3 \pm 0.2$ was measured, and no significant differences in pressure between inferior vena cava, right atrium, and left atrium were found. Pulmonary and aortic flow were $4.9 \pm 0.6$ and $3.7 \pm 0.4 \mathrm{~L} / \mathrm{min}$, and pulmonary and systemic vascular resistance were $312 \pm 134$ and $2006 \pm 336$ dyne $\cdot \mathrm{s} \cdot \mathrm{cm}^{-5}$.

Conclusions: Left and right heart catheterization using MR guidance is feasible. The combination of hemodynamic catheterization data with anatomic and functional MRI may significantly improve the evaluation of patients with congenital heart disease while avoiding radiation exposure. 


\section{Introduction}

Cardiac catheterization procedures are used to precisely define anatomic and physiological abnormalities caused by various diseases. Cardiac catheterization comprises procedures that require introduction of catheters into the central arterial and/or venous system that are steered to specific cardiac chambers or vessels to obtain regional information or for delivering local therapies. In pediatric and adult patients, these procedures are routinely guided by x-ray fluoroscopy. Patients with congenital heart disease often undergo multiple cardiac catheterizations that can lead to accumulation of high radiation doses. ${ }^{1-3}$ Diagnostic ionizing radiation is a definite risk factors for cancer development, especially in children. ${ }^{4}$

Despite their routine use, $x$-ray fluoroscopy and angiography do not provide 3D anatomic information, which can be crucial in patients with complex congenital heart disease. In contrast, MRI is characterized by 3D anatomic information in various imaging planes without the exposure to ionizing radiation for either the patient or the operator. In addition, functional information such as quantitative blood flow and ejection fraction is provided. The introduction of fast $\mathrm{MRI}^{5}$ has made visualization of moving structures possible, including anatomic details in the rapidly beating heart. More recently, realtime imaging has been used for deployment of atrial septal defect closure devices, ${ }^{6}$ coronary and pulmonary stenting, ${ }^{7,8}$ and balloon angioplasty of peripheral ${ }^{9}$ or renal arteries. ${ }^{10}$ MR catheter guidance, if effective, would eliminate ionizing radiation exposure associated with catheterization. Thus, the purposes of this study were to (1) guide left and right heart catheterization with real-time MRI using venous, venoustransseptal, and arteriovascular access in a swine model of acute atrial septal defect; (2) simultaneously measure intracardiac and great artery pressures and oxygen saturations; and (3) determine pulmonary blood flow, cardiac output, and pulmonary and systemic resistance.

\section{Methods}

\section{XMR System}

Experiments were performed with a hybrid XMR system (Philips Medical Systems) incorporating a digital x-ray Integris V5000 angiography-catheterization laboratory with an adjoining 1.5-T Intera MR scanner. The suites were connected by a moving tabletop for rapid transport of the animals from $\mathrm{x}$-ray to MR system. X-ray fluoroscopy was needed for the preparation of the animal model before initiation of cardiac catheterization. 


\section{Animal Model}

Female domestic farm pigs ( $n=7,37$ to $43 \mathrm{~kg}$; age, 3 months) were studied in accordance with the National Institutes of Health Guide for the Care and Use of Laboratory Animals and with approval of the Committee of Animal Research of the University. After medication with intramuscular injection of $0.04 \mathrm{mg} / \mathrm{kg}$ atropine, $2 \mathrm{mg} / \mathrm{kg}$ xylazine, and $20 \mathrm{mg} / \mathrm{kg}$ ketamine, general anesthesia was initiated with $2 \%$ inhalation isoflurane. Animals were intubated and mechanically ventilated. Local anesthesia with $2 \%$ lidocaine was injected subcutaneously before placement of $9 \mathrm{~F}$ femoral arterial and $12 \mathrm{~F}$ femoral venous introducer sheaths. After vascular access had been established, a needle followed by a balloon catheter (18-mm diameter) was used to puncture the atrial septum and subsequently dilate the defect under $x$-ray fluoroscopic guidance to allow later transseptal catheter steering. Once the septostomy had been performed, $\mathrm{x}$-ray fluoroscopy was no longer used in the study.

\section{MR Guidance of Cardiac Catheterization}

After transfer to the MR system, an active tracking catheter (Philips Medical Systems/Cordis Corp; Figure 1) was introduced via femoral vein. A copper microcoil mounted on the catheter tip was projected continuously as a small cross on MR images (Figures 2 to 6). The microcoil was connected to an external preamplifier box by copper leads. An external plastic sheath was added over the catheter to isolate the components. Because the catheter shaft was seen only occasionally on MR images, steering of the catheter would not be possible without active imaging of the tip. The catheter was guided to the inferior vena cava (IVC), right atrium (RA), right ventricle (RV), and main pulmonary artery (PA). After withdrawal to the RA, the catheter was steered across the septal defect from RA to left atrium (LA) and to the left ventricle (LV). In addition, the catheter introduced into the femoral artery was also advanced retrogradely into the aorta and LV. A pulmonary vein and superior vena cava were also catheterized in 2 animals and the brachiocephalic artery in 1 . In all areas of interest, pressure curves and blood samples to determine oxygen saturation and hemoglobin levels were obtained.

\section{MRI Techniques}

Real-time image acquisition was performed to image simultaneously catheter tip position and background anatomy by use of a real-time steady-state free precession sequence (SSFP with radial k-space filling: TR, $3 \mathrm{~ms}$; TE, $1.5 \mathrm{~ms}$; flip angle, $60^{\circ}$; slice thickness, $10 \mathrm{~mm}$; field of view, $200 \mathrm{~mm}$; scan matrix, 128x128; $300 \mathrm{~ms}$ per image, reconstructed at 10 frames/s using sliding-window reconstruction [view sharing] ${ }^{11,12}$ ). A 2element phased array of surface coils (2 circular 20-cm-diameter coils) was used. The number of trajectories for an image set was 102. However, image reconstruction was 
performed every 34 radial profiles by use of the sliding-window technique. Latency associated with reconstruction and image display was $<50 \mathrm{~ms}$. The interactive scan plane adjustment was performed on the fly, with the time to realize a new plane being just more than the full data acquisition time (300 ms). Planes could be adjusted manually or tracked automatically to include the tip of the active tracking catheter. Catheter tip movements within an imaging plane (in-plane motion) were tracked continuously in real-time, whereas through-plane tip tracking was updated on an "on-demand" basis. Neither respiratory navigator techniques nor cardiac triggering was necessary.

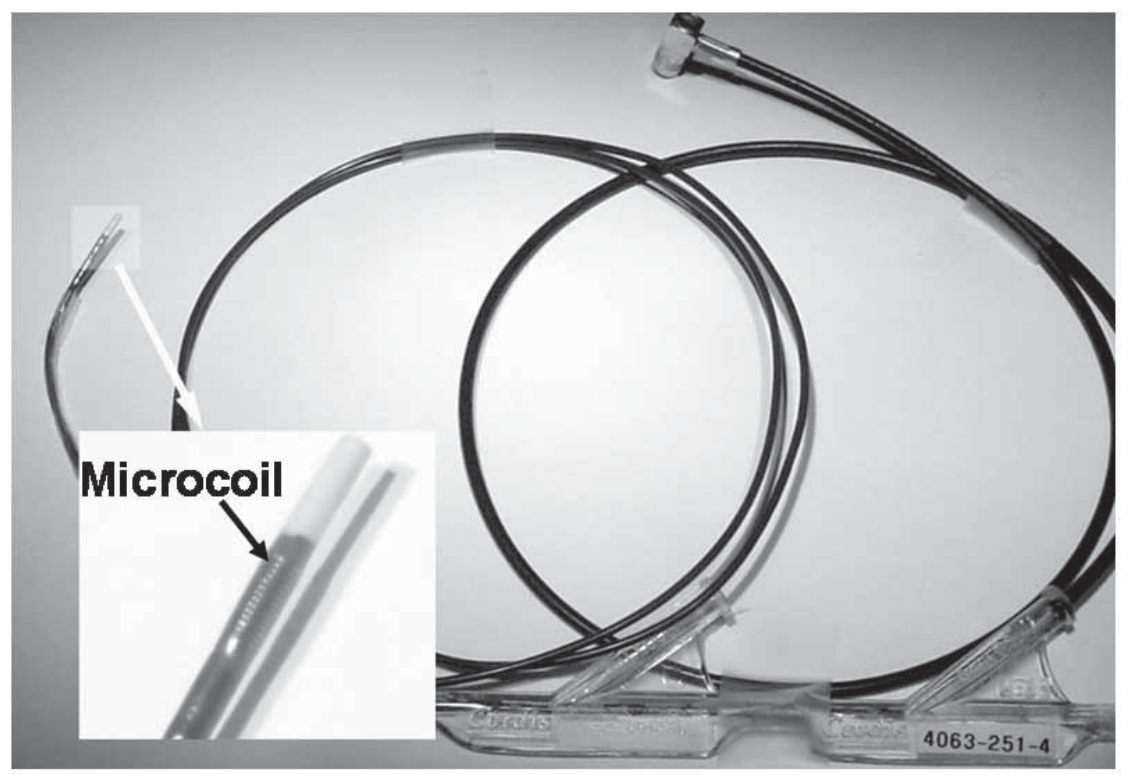

Figure 1: Active tracking catheter prototype. $6 \mathrm{~F}$ tracking catheter was $90 \mathrm{~cm}$ long and contained a tracking microcoil near its tip (enlargement). A transmission line exits proximal end of catheter and was used to connect microcoil to MR scanner so that its position could be tracked automatically.

To calculate pulmonary and systemic vascular resistance, blood flows in the main PA and aorta were measured by use of a retrospectively gated velocity-encoding phasecontrast gradientecho sequence (TR, $15 \mathrm{~ms}$; TE, $4 \mathrm{~ms}$; flip angle, 20; scan matrix, 192x192; slice thickness, $8 \mathrm{~mm}$; field of view, $200 \mathrm{~mm}$ ). Velocity-encoded range was $\pm 150 \mathrm{~cm} / \mathrm{s}$ for PA and $\pm 200 \mathrm{~cm} / \mathrm{s}$ for aorta. Pulmonary and systemic vascular resistance in dyne $\cdot \mathrm{s} \cdot \mathrm{cm}^{-5}$ were calculated from flow and pressure measurements by use of the formula PVR=80(PA mean-LA mean)/Qp and SVR=80(Aorta mean-RA mean)/Qs, where Qp is pulmonary flow per minute and Qs is aortic flow per minute. Left-to-right shunt calculations are given as the Qp/Qs ratio calculated from MR flow measurements. In addition, the $\mathrm{Qp} / \mathrm{Q}$ s ratio was also calculated from oxygen saturation measurements by use of the following equation: 
$\frac{Q p}{Q s}=\frac{\text { saturation }_{\mathrm{Aorta}}-\text { saturation }_{\mathrm{IVC}}}{\text { saturation }_{\mathrm{LA}}-\text { saturation }_{\mathrm{PA}}}$

Postmortem Analysis

At the conclusion of the study, animals were euthanized with $150 \mathrm{mg} / \mathrm{kg}$ pentobarbital IV. After removal of the heart and adjacent great vessels, visual inspection was performed to assess for pericardial effusion, perforation, and mural hematoma.

Statistical Analysis

All data are expressed as mean_SD. The paired Student's $t$ test was used to compare between pressures or oxygen saturations obtained at different regions of interest. Linear regression was calculated to determine the correlation between Qp/Qs ratios obtained from flow and oxygen saturation measurements.

\section{Results}

The catheter tip was placed in the areas of interest to obtain pressure curves and blood samples in all animals. During the procedure, there were no significant arrhythmias, perforations, hemorrhages, or other complications. Correct catheter placement was proved by MR images and the corresponding pressure curves. Time for introducing the catheter and steering to IVC, RA, superior vena cava, RV, PA, LA, pulmonary vein, and LV ranged from 10 to 30 minutes, with a decrease with increasing experience.

The image planes used to guide the catheter tip into each of the cardiac chambers and great vessels are shown in Figures 2 to 6 . With MR, an infinite array of imaging planes can be acquired. For passage of the catheter from one site to the next chamber or vessel, 1 or more planes were selected that showed the catheter tip and the chamber or vessel into which the tip was to be passed. The resulting multiple imaging planes were named by the 2 structures of interest shown in each plane (IVC-RA, RV-PA, RA-LA) unless standard planes such as cardiac 4-chamber or short-axis views were used. After the catheter had been advanced along the IVC, an IVC-RA plane (Figure 2a) was used for steering the catheter from the IVC into the RA. The image plane was then switched to a 4-chamber view of the heart (Figure $2 b$ ) to steer the catheter across the tricuspid valve into the RV. To advance the catheter into the PA (Figure 3), both a cardiac short-axis view (Figure 2c) and a 4-chamber view were used for positioning of the catheter near the RV outflow tract. After switching to the RV-PA planes (Figure 2, $d$ and e), the catheter was advanced across the pulmonary valve into the main PA. 

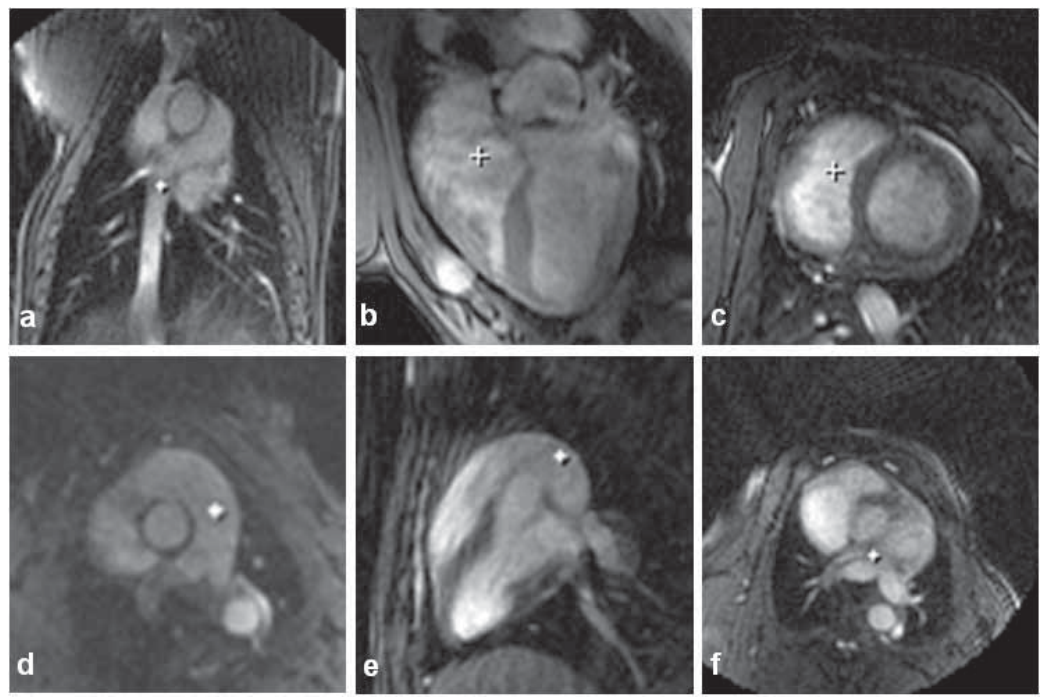

Figure 2: Menu of different image planes obtained with real-time SSFP imaging. Images a-f are image planes used for catheter steering to regions of interest. IVC-RA image plane (a) shows IVC, RA, and LA and was used for advancing catheter along IVC into RA junction and RA. Image plane was then switched to a cardiac 4-chamber (long-axis) view (b) to steer catheter across tricuspid valve into RV. To advance catheter into PA, cardiac short-axis (c) and 4-chamber (b) views were acquired sequentially for positioning of catheter near RV outflow tract. Subsequently, catheter was advanced across pulmonary valve into main PA using RV-PA image planes ( $d$ and e). For antegrade left heart catheterization, a, RA-LA image plane (f) was deployed in addition to a cardiac 4-chamber view (b).

For antegrade catheterization of the left heart, the catheter was advanced from the IVC-RA junction to the RA and transseptal to the LA by use of an RA-LA view plane (Figure 2f). A 4-chamber imaging plane (Figures $2 \mathrm{~b}$ and 4 ) was used to steer the catheter across the mitral valve into the LV. For retrograde left heart catheterization, an image plane showing the aortic arch and LV (Figure 5) was used to advance the catheter along the aortic arch across the aortic valve into the LV. Imaging planes for catheter guidance into the pulmonary veins are shown in Figure 6.

At postmortem, visual inspection of animal hearts and great vessels revealed no evidence of pericardial effusion, perforation, hematoma, or injuries. The presence of atrial septal defect was confirmed in all animals. 

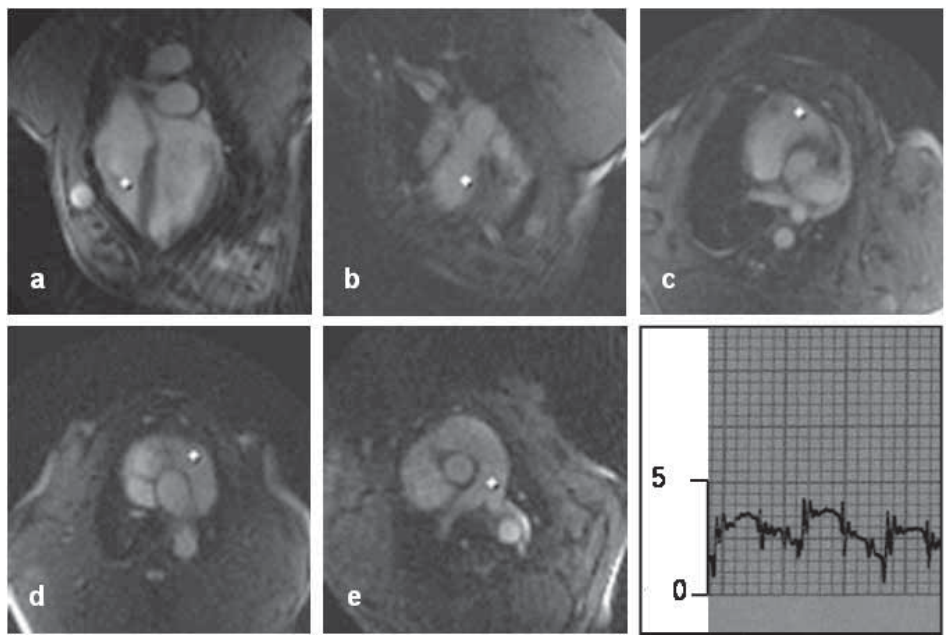

Figure 3: Catheterization of main PA with real-time SSFP MRI guidance. Image planes (4chamber [a], right ventricular outflow tract [b], and 3 outflow-tract-PA planes [c-e]) used for catheter steering and a PA pressure curve in $\mathrm{mm} \mathrm{Hg}$ are shown.
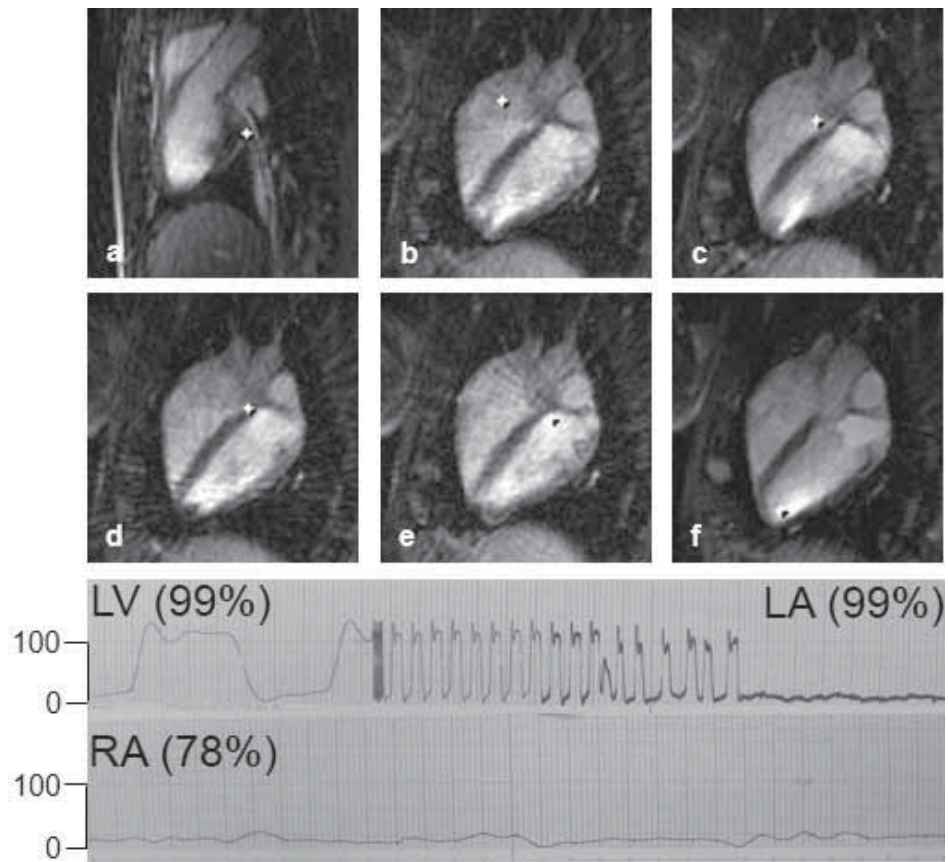

Figure 4: Antegrade catheterization of LV (femoral venous/transseptal access) with realtime SSFP MRI guidance. MR images in top row show advancement of tracking catheter from IVC into RA (a-c), transseptal into LA (d, e), and LV (f). Catheter tip is detected as an overlaid cross on real-time SSFP images. During catheter pullback, pressure curves in $\mathrm{mm} \mathrm{Hg}$ were recorded from LV, LA, and RA. Oxygen saturation in percent for LV, LA, and RA is given in parentheses. 

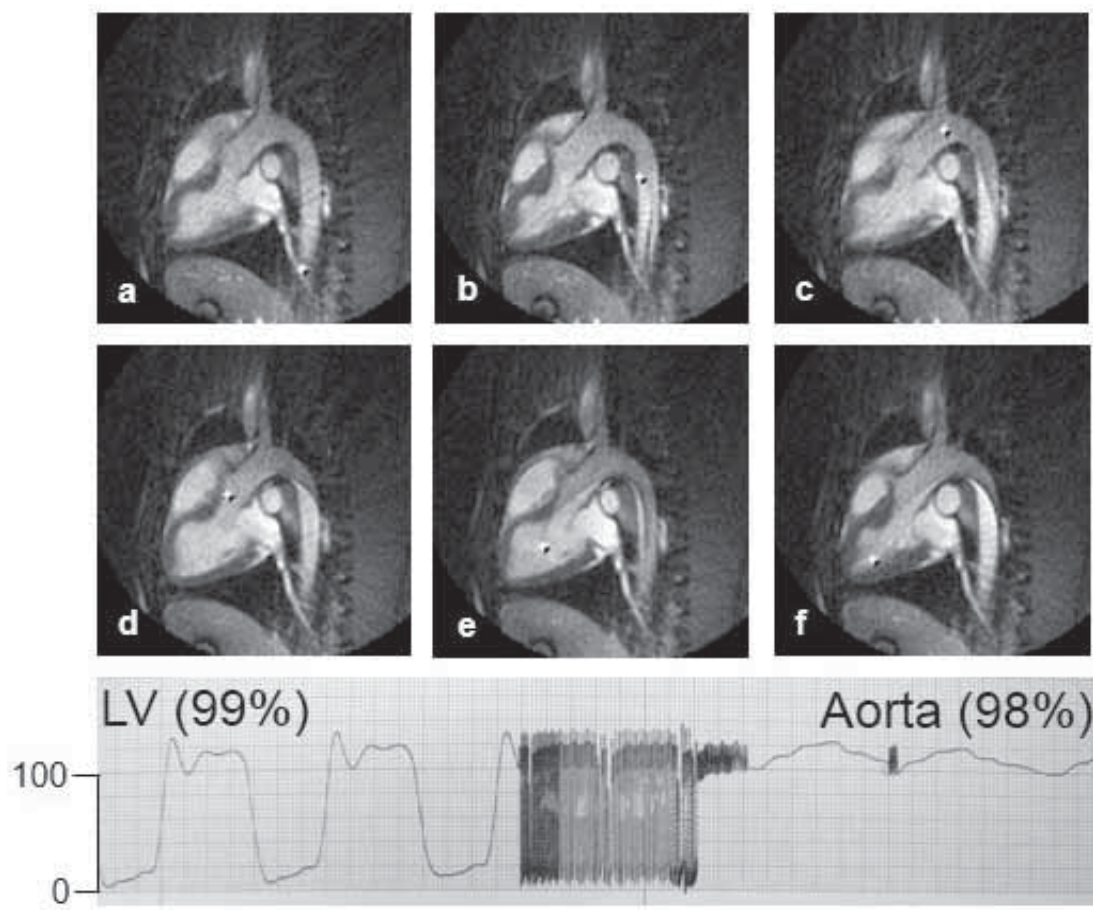

Figure 5: Retrograde catheterization of LV (femoral arterial access) with real-time SSFP imaging guidance. Top row $(\mathrm{a}-\mathrm{c}), \mathrm{MR}$ images show tracking catheter advanced from descending aorta to transverse aortic arch. Bottom row $(\mathrm{d}-\mathrm{f})$, catheter moving across aortic valve to apex of LV. Catheter tip is visualized as a cross superimposed on real-time MR images, whereas catheter shaft is seen only in parts. During catheter pullback, pressure curves in $\mathrm{mm} \mathrm{Hg}$ were recorded from LV and ascending aorta (Aorta). Oxygen saturation in percent for LV and Aorta is given in parentheses.
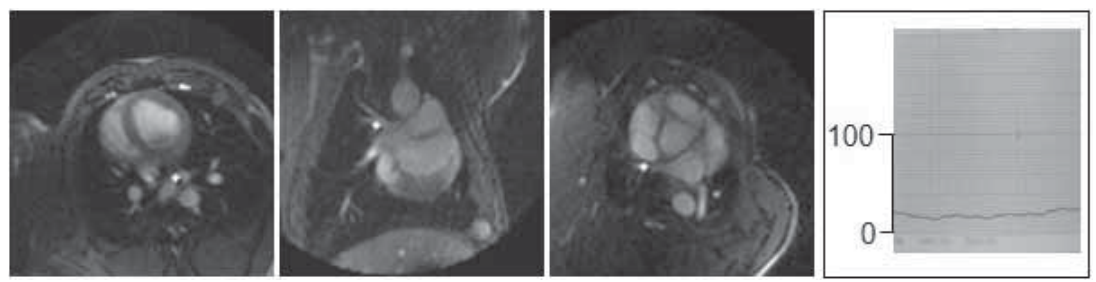

Figure 6: Catheterization of a pulmonary vein with real-time SSFP MRI guidance. Three different image planes were used for guidance of catheter into right upper pulmonary vein. Catheter tip is superimposed as a blinking cross on real-time images. A pressure recording is shown on right.

\section{Catheterization Data}

Pressures, oxygen saturation, and hemoglobin levels are shown in the Table. Mean heart rate was $87 \pm 11 \mathrm{bpm}$. There were no significant differences in pressure between 
RA and IVC ( $P=0.23)$ or LA ( $P=0.22)$. No significant differences in oxygen saturation were found between IVC and RA ( $P=0.34)$ or RV $(P=0.21)$.

$\mathrm{PA}$ and aortic flows determined by MR phase-contrast technique were $4.9 \pm 0.6$ and $3.7 \pm 0.4 \mathrm{~L} / \mathrm{min}$, indicating a left-to-right shunt of $1.2 \pm 0.6 \mathrm{~L} / \mathrm{min}$ and a $\mathrm{Qp} / \mathrm{Qs}$ ratio of 1.3 \pm 0.2 . Calculation of $Q p / Q$ s from the oxygen saturation measurements was $1.4 \pm 0.4$, similar to MR flow measurements $(r=0.68, \mathrm{SEM}=0.32$, regression line equation $y=1.39 \mathrm{x}$ -0.45). Pulmonary and systemic vascular resistance values derived from MR flow data and pressure measurements were $312 \pm 134$ and $2006 \pm 336 \mathrm{dyne} \cdot \mathrm{s} \cdot \mathrm{cm}^{-5}$.

Table 1: M easurement data from different regions of interest

\begin{tabular}{lccccccc}
\hline & \multicolumn{7}{c}{ Region of interest } \\
\cline { 2 - 8 } Parameter & IVC & RA & RV & PA & LA & LV & Aorta \\
\hline $02, \%$ & $61 \pm 11$ & $67 \pm 12$ & $69 \pm 13$ & $68 \pm 10$ & $94 \pm 11$ & $94 \pm 11$ & $96 \pm 3$ \\
$\mathrm{Hb}, \mathrm{mg} / \mathrm{dl}$ & $10.5 \pm 1.1$ & $10.8 \pm 0.6$ & $11.2 \pm 2.0$ & $10.6 \pm 1.1$ & $10.5 \pm 1.0$ & $10.8 \pm 1.1$ & $10.8 \pm 1.2$ \\
$\mathrm{P}, \mathrm{mmHg}$ & $9 \pm 1$ & $11 \pm 1$ & $43 \pm 7 / 1 \pm 1 / 8 \pm 2$ & $43 \pm 8 / 24 \pm 6$ & $12 \pm 2$ & $131 \pm 16 / 3 \pm 3 / 11 \pm 3$ & $132 \pm 17 / 104 \pm 22$ \\
\hline
\end{tabular}

Values are mean \pm standard deviation. $\mathrm{O}_{2}$ indicates oxygen saturation; $\mathrm{Hb}$, hemoglobin level. $\mathrm{P}$ indicates pressure: mean pressure for inferior vena cava (IVC), right (RA) and left atrium (LA); systolic and diastolic pressure for pulmonary artery (PA) and ascending aorta (Aorta); systolic, early and end-diastolic pressure for right (RV) and left ventricle (LV).

\section{Discussion}

The major finding of the present study was that left and right heart catheterization is feasible under MR real-time guidance. This study represents an early step toward MRguided diagnostic cardiac catheterization. Active catheter tip tracking was highly useful in steering and in selecting regions of interest by use of venous, venoustransseptal, and arterial approaches. To the best of our knowledge, this is the first study to show the feasibility of MR fluoroscopy for left and right heart catheterization with assessment of hemodynamic parameters. MR flow data in combination with blood pressure and oxygen saturation measurements can provide additional insight into systemic and pulmonary flows and intracardiac shunts and can be applied to calculation of pulmonary and systemic vascular resistances. The study results were similar to those of previously reported results based on the standard method of thermodilution and pressure measurements. $^{13}$

The flow volume through the shunt in our atrial septal defect model was small, as was expected for an acutely created defect. Kong et al $^{14}$ also observed an increase of the Qp/Qs ratio from $1.03 \pm 0.12$ to only $1.28 \pm 0.23$ after creating an acute atrial septal defect in pigs. Calculations of Qp/Qs on the basis of oxygen saturation measurements were consistent with the MR flow data in the present study. 
Indications for diagnostic cardiac catheterization in pediatric cardiology are to evaluate congenital heart disease with complex anatomy that cannot be assessed well with echocardiography, such as abnormalities of the pulmonary arteries and the aortic arch. Both regions can be assessed with MRI or MR angiography. ${ }^{15-18}$ Further indications for cardiac catheterization in children are pressure, oxygen saturation, and flow measurements to define the severity of valvular or vascular stenosis and to define ventricular diastolic and systolic function and pulmonary vascular resistance. In the present study, these values were obtained from MR flow measurements and blood pressure and oxygen saturation data. Cine MRI can be used to obtain additional functional indices during MR-guided cardiac catheterizations, including wall motion analysis ${ }^{19}$ and LV and RV volumes. $^{20,21}$

The success of cardiac catheterization depends in part on the ability to visualize catheter and central cardiovascular anatomy simultaneously and in real-time. A hypothesis explored in this study was whether real-time MRI could provide this ability without radiation exposure. Indeed, MR fluoroscopy in a variety of different imaging planes displayed both cardiovascular anatomy and the catheter tip position in real-time. Rapid switching among various imaging planes during the procedure was successful, allowing precise catheter manipulation to the target region of interest.

MR catheter guidance totally eliminates radiation exposure. With x-ray fluoroscopy, cardiac catheterization and long interventional procedures could result in relatively high radiation exposure for patient and staff. It has been estimated that per hour of fluoroscopy, there is a $0.07 \%$ to $0.1 \%$ lifetime risk of developing a fatal malignancy and 1 to 20 genetic defects per 1 million births,2,3 risks especially important for pediatric patients. $^{1,22}$

High temporal $(300 \mathrm{~ms})$ and spatial $(1.6 \mathrm{~mm})$ resolution steady-state free precession images were acquired in real-time to monitor catheter steering and visualize background anatomy. The imaging speed of 3 frames/s (reconstructed at 10 frames/s) was sufficient for catheter guidance. Blood appeared bright, resulting in a good contrast between vessel lumen and surrounding tissue. Catheter artifacts from the shaft were small and did not superimpose on vascular or chamber walls. However, despite the visualization of the catheter tip during the entire procedure, adjacent parts of the catheter were not reliably visualized. The poor visualization of the catheter shaft can be attributed to out-of-plane movements resulting from cardiac contraction and respiratory motion and the fact that optimal contrast between blood and catheter was not always achieved. Because the slice thickness was $10 \mathrm{~mm}$, distal bending or angulation of the catheter shaft also resulted in a loss of visualization. Imaging large parts of a catheter would be preferable to merely tracking the tip. To keep the catheter in plane, a larger slice thickness may be needed in some locations. In addition, for steering purposes, not only the accurate position of the catheter tip but also visualization of the direction of angulation of the catheter tip are important. As suggested by Zhang et al, ${ }^{23}$ mul- 
tiple-element coils at different positions near the catheter tip should provide this visualization.

The introduction of MR-guided catheterization into clinical practice depends critically on the development and availability of MR-safe catheters. Radiofrequency-induced heating might occur even in nonferromagnetic metals when used in catheters or guidewires. No guidewires were used in this study, but the catheter prototype contained electrical conductors. No sparking was observed, and no perceptible heating or other adverse effects on the animals were recognized. However, further studies are needed to systematically assess MR safety and optimize catheter prototypes, because heating of guidewires was observed in vitro. ${ }^{24,25}$ A preliminary animal study about MR safety of guidewires showed no thermal damage or blood coagulation, ${ }^{26}$ but the safety of active tracking catheters with long electrical conductor cables is not known. Using fiberoptic instead of electrical conductors in active tracking catheters might be a strategy to avoid heating. ${ }^{27,28}$ The concept of passive tracking with susceptibility markers on a catheter, ${ }^{29,30}$ contrast media-filled catheters, or contrast media-filled balloons at the catheter tip ${ }^{31}$ is considered a safer approach, but imaging at fast speed with good anatomic information and optimal visualization of the marker's susceptibility artifacts is at present difficult. $^{32}$

In conclusion, left and right heart catheterization using real-time MR guidance is feasible. It has the potential to change the current $x$-ray- based diagnostic approach for children with congenital heart disease to avoid radiation exposure. Hemodynamic catheterization data can be combined with anatomic and functional MRI. This may significantly improve the evaluation of patients with complex congenital heart disease. Introduction of MR-guided catheterization into clinical practice depends on the development and availability of MR-compatible catheters.

\section{References}

1. Wu JR, Huang TY, Wu DK, et al. Radiation exposure of pediatric patients and physicians during cardiac catheterization and balloon pulmonary valvuloplasty. Am J Cardiol. 1991;68:221-225.

2. Calkins $\mathrm{H}$, Niklason L, Sousa J, et al. Radiation exposure during radiofrequency catheter ablation of accessory atrioventricular connections. Circulation. 1991;84:2376-2382.

3. Perisinakis K, Damilakis J, Theocharopoulos N, et al. Accurate assessment of patient effective radiation dose and associated detriment risk from radiofrequency catheter ablation procedures. Circulation. 2001;104:58-62.

4. Modan B, Keinan L, Blumstein T, et al. Cancer following cardiac catheterization in childhood. Int J Epidemiol. 2000;29:424-428.

5. Yang PC, Kerr AB, Liu AC, et al. New real-time interactive cardiac magnetic resonance imaging system complements echocardiography. J Am Coll Cardiol. 1998;32:2049-2056.

6. Buecker A, Spuentrup E, Grabitz R, et al. Magnetic resonance-guided placement of atrial septal closure device in animal model of patent foramen ovale. Circulation. 2002;106:511-515.

7. Spuentrup E, Ruebben A, Schaeffter T, et al. Magnetic resonance- guided coronary artery stent placement in a swine model. Circulation. 2002;105:874-879. 
8. Kuehne T, Saeed M, Higgins CB, et al. Endovascular stents in pulmonary valve and artery in swine: feasibility study of MR imaging-guided deployment and postinterventional assessment. Radiology. 2003;226:475-481.

9. Wildermuth S, Debatin JF, Leung DA, et al. MR imaging-guided intravascular procedures: initial demonstration in a pig model. Radiology. 1997;202:578 -583.

10. Omary RA, Frayne R, Unal O, et al. MR-guided angioplasty of renal artery stenosis in a pig model: a feasibility study. J Vasc Interv Radiol. 2000;11:373-381.

11. Riederer SJ, Tasciyan T, Farzaneh F, et al. MR fluoroscopy: technical feasibility. Magn Reson Med. 1988;8:1-15.

12. Foo TK, Bernstein MA, Aisen AM, et al. Improved ejection fraction and flow velocity estimates with use of view sharing and uniform repetition time excitation with fast cardiac techniques. Radiology. 1995;195:471-478.

13. Martini L, Fini M, Giavaresi G, et al. Haemodynamic and volumetric monitoring during haemorrhagic shock in swine. Resuscitation. 2001;51:69-76.

14. Kong $\mathrm{H}, \mathrm{Gu} X$, Titus JL, et al. Creation of an intra-atrial communication with a new Amplatzer shunt prosthesis: preliminary results in a swine model. Cathet Cardiovasc Interv. 2002;56:267-271.

15. Masui T, Katayama M, Kobayashi S, et al. Gadolinium-enhanced MR angiography in the evaluation of congenital cardiovascular disease pre and postoperative states in infants and children. J Magn Reson Imaging. 2000;12:1034-1042.

16. Holmqvist C, Hochbergs P, Bjorkhem G, et al. Pre-operative evaluation with MR in tetralogy of Fallot and pulmonary atresia with ventricular septal defect. Acta Radiol. 2001;42:63-69.

17. Godart F, Labrot G, Devos P, et al. Coarctation of the aorta: comparison of aortic dimensions between conventional MR imaging, 3D MR angiography, and conventional angiography. Eur Radiol. 2002;12:20342039.

18. Ohno Y, Hatabu H, Takenaka D, et al. Contrast-enhanced MR perfusion imaging and MR angiography: utility for management of pulmonary arteriovenous malformations for embolotherapy. Eur J Radiol. 2002;41:136-146.

19. Laham RJ, Simons M, Pearlman JD, et al. Magnetic resonance imaging demonstrates improved regional systolic wall motion and thickening and myocardial perfusion of myocardial territories treated by laser myocardial revascularization. J Am Coll Cardiol. 2002;39:1-8.

20. Lorenz $\mathrm{CH}$, Walker ES, Morgan VL, et al. Normal human right and left ventricular mass, systolic function, and gender differences by cine magnetic resonance imaging. J Cardiovasc Magn Reson. 1999;1:7-21.

21. Thiele H, Paetsch I, Schnackenburg B, et al. Improved accuracy of quantitative assessment of left ventricular volume and ejection fraction by geometric models with steady-state free precession. J Cardiovasc Magn Reson. 2002;4:327-339.

22. Moore JD, Shim D, Sweet J, et al. Radiation exposure to children during coil occlusion of the patent ductus arteriosus. Catheter Cardiovasc Interv. 1999;47:449-454.

23. Zhang $Q$, Wendt $M$, Aschoff AJ, et al. A multielement RF coil for MRI guidance of interventional devices. J Magn Reson Imaging. 2001;14:56-62.

24. Liu CY, Farahani K, Lu DS, et al. Safety of MRI-guided endovascular guidewire applications. J Magn Reson Imaging. 2000;12:75-78.

25. Konings MK, Bartels LW, Smits HF, et al. Heating around intravascular guidewires by resonating RF waves. J Magn Reson Imaging. 2000;12:79-85.

26. Yang $\mathrm{X}$, Yeung $\mathrm{C}$, Ji $\mathrm{H}$, et al. Thermal effect of intravascular MR imaging using an MR imaging-guidewire: an in vivo laboratory and histopathological evaluation. Med Sci Monit. 2002;8:MT113-MT117.

27. Wong EY, Zhang $Q$, Duerk JL, et al. An optical system for wireless detuning of parallel resonant circuits. J Magn Reson Imaging. 2000;12:632-638.

28. Konings MK, Bartels LW, van Swol CF, et al. Development of an MR-safe tracking catheter with a laserdriven tip coil. J Magn Reson Imaging. 2001;13:131-135.

29. Bakker CJ, Hoogeveen RM, Weber J, et al. Visualization of dedicated catheters using fast scanning techniques with potential for MR-guided vascular interventions. Magn Reson Med. 1996;36:816-820. 
30. Van der Weide R, Bakker CJ, Viergever MA. Localization of intravascular devices with paramagnetic markers in MR images. IEEE Trans Med Imaging. 2001;20:1061-1071.

31. Godart F, Beregi JP, Nicol L, et al. MR-guided balloon angioplasty of stenosed aorta: in vivo evaluation using near-standard instruments and a passive tracking technique. J Magn Reson Imaging. 2000;12:639644.

32. Bakker CJ, Bos C, Weinmann HJ. Passive tracking of catheters and guidewires by contrast-enhanced MR fluoroscopy. Magn Reson Med. 2001;45:17-23. 


\section{Balloon sizing and transcatheter closure of}

acute atrial septal defects guided by magnetic resonance fluoroscopy:

Assessment and validation in a large animal model 


\section{Abstract}

Purpose: To quantitatively assess atrial septal defects (ASDs) with small shunts using MR imaging followed by transcatheter closure monitored by MR fluoroscopy.

Materials and methods: Acute ASDs were created in 14 pigs under x-ray fluoroscopy. Six animals were studied in order to select MR-compatible delivery systems and imaging strategies. ASDs in 8 animals were examined with balloon sizing under MR fluoroscopy, flow measurements and contrast media injections, after which transcatheter closure was performed under MR fluoroscopy. The delivery system was assembled from commercially available materials.

Results: The ratio of pulmonary to systemic flow (Qp/Qs) was reduced from $1.23 \pm 0.15$ before to $1.07 \pm 0.11$ after ASD closure $(p<0.001)$. In 2 out of 8 animals Qp/Qs was close to 1.0 before closure despite the presence of defects $>15 \mathrm{~mm}$. The ASDs were measurable with MR balloon sizing in all of the animals. Balloon sizing was identical with MR $(16.9 \pm 2.3 \mathrm{~mm})$ and $x$-ray fluoroscopy $(17.1 \pm 1.3 \mathrm{~mm})$. The in-house-assembled delivery system allowed successful placement of closure devices under MR guidance.

Conclusions: Assessment and closure of small shunts with MR fluoroscopy is feasible. A barrier to the rapid implementation of transcatheter closure in patients is uncertainty about the MR safety of guidewires and device delivery systems. 


\section{Introduction}

Atrial septum defects (ASDs) are common congenital heart defects, occurring in $>3$ out of 10000 births. ${ }^{1}$ While these defects may be asymptomatic for years, resulting heart failure and pulmonary hypertension sometimes occur beyond the age of $30 .^{2}$ Defect closure is therefore routinely performed to improve cardiac function and prevent heart failure. X-ray fluoroscopy-guided transcatheter closure of ASDs has been successfully used in children and adults to avoid open-heart surgery ${ }^{3,4}$ and is rapidly becoming the treatment of choice. However, ionizing radiation is a risk factor for cancer development, especially in children. ${ }^{5}$ Some patients with congenital heart disease accumulate large doses of radiation during evaluation, interventional treatment, and follow-up studies. ${ }^{6,7}$ Magnetic resonance imaging (MRI) for the assessment of cardiac function and catheter guidance offers several advantages, including the elimination of radiation exposure for both the patient and the physician. It provides 3-dimensional anatomic information in variable imaging planes, which can be crucial for patients with complex congenital heart disease. Furthermore, functional information including quantitative measurements of blood flow and ventricular function can be obtained in a single imaging session.

The need for ASD closure in asymptomatic patients with small pulmonary to systemic flow (Qp/Qs) ratios is controversial. ${ }^{8}$ However, it remains difficult to detect and visualize ASDs with Qp/Qs ratios < 1.5 using MRI. ${ }^{9-11}$ Two recent papers demonstrated the potential of MRI for the deployment of ASD closure devices using specially designed prototype systems to prevent large susceptibility artifacts. ${ }^{12,13}$ The aims of the present study were to explore the potential of MRI to 1) depict acute ASDs with small shunts, 2) guide endovascular closure systems using active and passive MR catheter tracking, and 3) use commercially available materials that are not susceptible to MR artifacts for device delivery.

\section{Material and methods}

\section{Experimental model}

Fourteen domestic farm pigs were studied in accordance with the Guide for the Care and Use of Laboratory Animals (National Institutes of Health) and with the approval of the committee of animal research at our institution. We used 6 animals $(20-41 \mathrm{~kg})$ in the first phase in order to select MR compatible delivery systems, and improve catheter handling and imaging strategies. Subsequently, we examined 8 animals $(38-44 \mathrm{~kg})$ in the second phase to assess flow and balloon sizes, and guidance of transcatheter ASD closure.

A premedication of $0.04 \mathrm{mg} / \mathrm{kg}$ atropine, $2 \mathrm{mg} / \mathrm{kg}$ xylazine, and $20 \mathrm{mg} / \mathrm{kg}$ ketamine was injected intramuscularly. Inhalation isoflurane was used for general anaesthesia. 
The animals were intubated and mechanically ventilated with room air. A $12 \mathrm{~F}$ short sheath was placed in the right femoral vein, a $6 \mathrm{~F}$ short sheath was placed in the right or left jugular vein and in the left femoral artery. Blood pressure was measured in the left femoral artery. Oxygen saturations were obtained in the inferior vena cava (IVC), aorta, left atrium (LA) and main pulmonary artery (PA) after the ASD was created, and the ratio of pulmonary flow (Qp in liters/minute) versus systemic flow (Qs in liters/minute) was calculated (Saturation Aorta - Saturation ${ }_{I V C}$ ) / $\left(\right.$ Saturation $_{L A}-$ Saturation $_{P A}$ ) to compare with the ratio of pulmonary systemic flow (Qp/Qs) obtained from MR flow measurements.

\section{$X$-ray and MR suite}

Experiments were performed with a hybrid XMR system (Philips Medical Systems, Best, The Netherlands). The system consisted of a $1.5 \mathrm{~T}$ Intera MR scanner connected by a tabletop to an adjoining digital x-ray Integris V5000 angiography-catheterization laboratory. A sliding door between the 2 systems shielded against $x$-ray radiation and radiofrequency leakage, and thus permitted combined or independent use of the suites. The sliding tabletop allowed rapid transport of the animals between $\mathrm{x}$-ray and MR systems.

\section{X-ray fluoroscopy}

X-ray fluoroscopy was only employed to create acute ASDs in the swine model and to compare initial balloon sizing and oxygen saturation with MR data. A needle catheter was used to establish an acute ASD. The defect was subsequently dilated with a balloon catheter (18mm diameter) filled with iodine contrast media (20\% stock solution, Omnipaque, Amersham Inc., Princeton, NJ) to allow visualization on x-ray fluoroscopy. We then assessed the defect using a sizing balloon catheter. We measured the size of the balloon on a screen using a standard analyzing software program (Easy Vision, Philips Medical Systems), after we calibrated for catheter diameter.

\section{MR flow measurements}

After the animals were transferred to the MR suite, a 2-element phased array of surface coils ( 2 circular $20 \mathrm{~cm}$-diameter coils) was placed around the thorax of the animals, which lay in the decubitus position. ECG was used to monitor heart rate and to perform retrospective gating of blood flow measurements. Velocity encoded cine MR flow measurements (retrospectively gated, velocity encoded, phase contrast, gradient echo sequence with TR/TE $=15 / 4 \mathrm{~ms}$, flip angle $20^{\circ}$, scan matrix $192 \times 192$, slice thickness $8 \mathrm{~mm}$, FOV $200 \mathrm{~mm}$ ) of the main PA and ascending aorta were performed before and after the ASD was closed. The imaging planes for flow measurements were perpendicular to the long axis of PA and aorta, respectively. Velocity-encoding range was set at $\pm 200 \mathrm{~cm} / \mathrm{s}$ 
for the aorta and $\pm 150 \mathrm{~cm} / \mathrm{s}$ for the PA. The pulmonary and aortic flow measurements were used to calculate the intracardiac shunt Qp/Qs ratio.

MR imaging of centrally injected Gd-DTPA-BMA

In animals without a detectable shunt by flow measurements, Gd-DTPA-BMA (10 ml of $20 \%$ of stock solution, Omniscan, Amersham Inc., Princeton, NJ) was injected in the main PA to confirm the absence of left-to-right shunting through the ASD. A T1weighted gradient echo sequence $\left(T R / T E=3.8 / 1.4 \mathrm{~ms}\right.$, flip angle $30^{\circ}$, scan matrix 192x192, slice thickness $8 \mathrm{~mm}$, FOV 200mm) was used to monitor shunting of the contrast agent. We chose to use central contrast media injection in order to achieve a compact bolus of contrast media. Therefore, these animals were transferred to the $x$ ray part so that a catheter could be placed in the right PA under x-ray fluoroscopy.

\section{MR Balloon Sizing}

The ASDs were assessed with balloon sizing under real-time MRI. The sizing balloon was advanced across the defect under MR fluoroscopy and inflated with Gd-DTPA-BMA (10\% of stock solution). Maximal distal and waist diameters were measured and compared with values obtained from $\mathrm{x}$-ray images and defect size postmortem. The same real-time MRI sequence was used for balloon sizing as described below.

Device delivery system

The device delivery system was assembled from commercially available materials consisting of an Amplatzer delivery sheath with a dilator (tip cut off) (AGA Medical Corp., Golden Valley, MN), size 18 Amplatzer septal occluder (Nitinol wires woven into 2 flat discs with a connecting waist and a polyester filling), a nitinol snare wire and a snare catheter (Microvena, White Bear Lake, MN) (Figure 1). The snare wire holding the device button was retracted against the snare catheter and dilator. The snare wire was secured in place by a plastic clamp in front of the dilator hub. Thus, the folded device could be advanced or withdrawn inside and out of the sheath by pushing or pulling the dilator-snare wire system. 


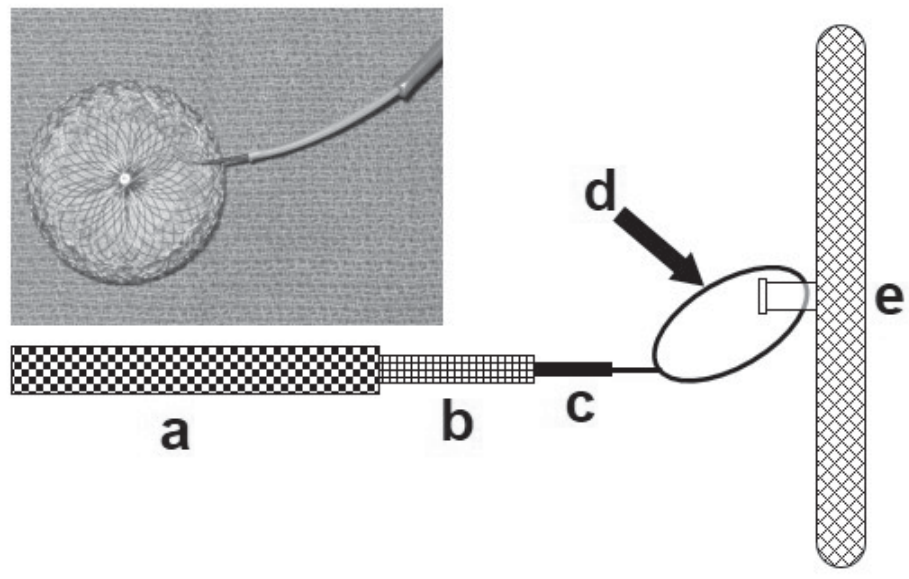

Figure 1: ASD closure device delivery system consisting of an Amplatzer delivery sheath (a) with dilator (tip cut off) (b), Amplatzer septal occluder (e), a nitinol snare wire (d) and a snare catheter (c). The snare wire holding the device button was retracted against the snare catheter and dilator. The snare wire was secured in place by a plastic clamp in front of the dilator hub. Thus, the folded device could be advanced or withdrawn inside and out of the sheath by pushing or pulling the dilator-snare wire system.

\section{Active and passive catheter tracking with MR imaging}

Two different methods were tested to position the delivery sheath across the ASD: active and passive catheter tracking. In 4 animals, a prototype active tracking catheter (Philips Medical Systems/Cordis Corp., Miami, FI) was used. The catheter tip was seen as a white cross superimposed on real-time MR images. Catheter tip movements within an imaging plane (in plane motion) were continuously tracked in real-time, while throughplane tip tracking was updated "on-demand". The catheter was steered from the IVC to the right atrium (RA), and then transseptally across the defect into the LA for the placement of a guidewire $(0.6 \mathrm{~mm}$ diameter, Terumo Corp., Tokyo, Japan) over which the delivery sheath was subsequently advanced into the LA. The sheath was advanced into the vessel the same distance as the tracking catheter. A real-time, steady state free precession imaging sequence was used for image acquisition (radial k-space filling; $\mathrm{TR} / \mathrm{TE}=3 / 1.5 \mathrm{~ms}$; flip angle $60^{\circ}$; slice thickness $10 \mathrm{~mm}$; FOV $200 \mathrm{~mm}$; scan matrix $128 \times 128$; $300 \mathrm{~ms}$ per image, reconstructed at 10 frames/s using the sliding window reconstruction (view sharing).

In 4 animals, passive tracking was employed. The delivery sheath with a dilator was steered directly transseptally across the defect under real-time MR guidance. The sheath was visualized as a low intensity structure within the high intensity of the blood pool. All components of the delivery device also produced low intensity signal (suscep- 
tibility artifact). After the dilator was withdrawn, the folded closure device was introduced into the sheath and advanced to the tip. The first disk was then unfolded into a balloon shape by pushing the snare catheter-dilator further in. The sheath was then pulled backwards until the disk reached the septum. The second disk was unfolded by further retracting the sheath over the snare wire / device. The device was then released by loosening the clamp and subsequently the snare wire. Finally. The snare wire, snare catheter, and dilator were removed from the delivery sheath.

\section{Postmortem examination}

After the closure device was placed, and the final MR flow measurements were taken, the animals were euthanized with $200 \mathrm{mg} / \mathrm{kg}$ pentobarbital administered intravenously. The heart and adjacent great vessels were excised. Visual inspection was performed to assess for correct position of the device, as well as to detect procedure related vascular or cardiac injuries, mural hematoma, perforation or pericardial effusion. In addition, balloon sizing was performed postmortem.

\section{Statistical analysis}

Data were expressed as the mean value \pm standard deviation. Qp/Qs results before and after ASD closure were compared with the use of a paired Student's $t$-test. Linear regression was calculated to determine the correlation between balloon sizing with MRI and $\mathrm{x}$-ray fluoroscopy, and postmortem, as well as the Qp/Qs ratios from the MR flow and oxygen saturation data. Absolute differences between different techniques were calculated according to Bland-Altman. ${ }^{14}$

\section{Results}

Assessment of ASD

The aortic flow before and after ASD closure was 3.75 $\pm 0.76 \mathrm{l} / \mathrm{min}$ and $3.13 \pm 1.01 \mathrm{l} / \mathrm{min}$ while pulmonary flow was $4.57 \pm 0.89 \mathrm{l} / \mathrm{min}$ and $3.29 \pm 0.98 \mathrm{l} / \mathrm{min}$. An example of phasic flow in the PA and aorta before and after defect closure is given in figure 2. 

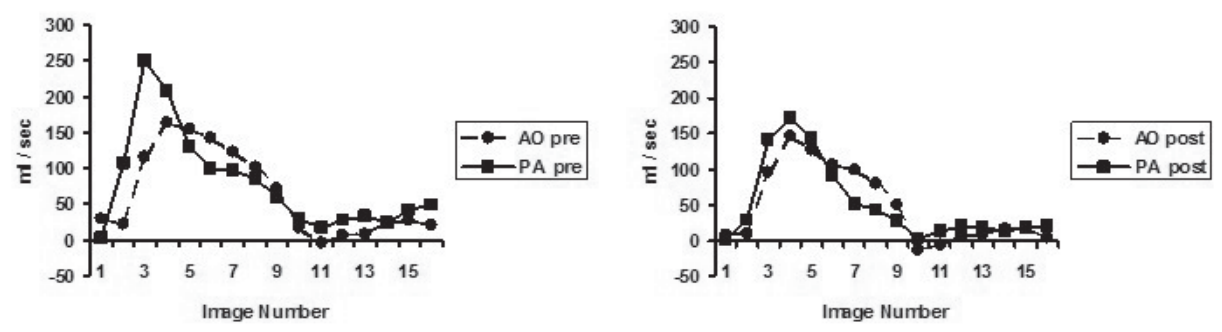

Figure 2: Example of phasic flow in the aorta and PA determined with velocity-encoded cine MRI before and after ASD closure. The pulmonary flow (PA) is greater than the aortic flow (AO) before (pre) defect closure (left), and the PA and AO become similar after (post) defect closure (right).

The Qp/Qs ratio was significantly reduced from $1.22 \pm 0.15$ before ASD closure to $1.07 \pm 0.11$ after ASD closure $(p<0.001)$. The range of the Qp/Qs ratio was 1.05 to 1.4 (median 1.24) before ASD closure and 0.92 to 1.27 (median 1.06) after closure. Oxygen saturation measurements revealed a Qp/Qs ratio before ASD closure of $1.29 \pm 0.31(r=$ 0.76 ; standard error of the estimate $=0.22$, regression line equation: $y=1.59 x-0.66$ for oxygen saturation versus MR flow measurements; absolute differences: $0.06 \pm 0.22$ ) (table 1).

Table 1: Measurements in 8 animals with acute ASDs.

\begin{tabular}{|c|c|c|c|c|c|c|c|c|c|c|}
\hline Animal & $\begin{array}{c}\mathrm{HR} \\
{[/ \mathrm{min}]}\end{array}$ & $\begin{array}{l}\text { BP Syst. } \\
\text { /Diast. } \\
\text { [mmHg] }\end{array}$ & $\begin{array}{c}\text { Aortic Flow } \\
{[\mathrm{ml} / \mathrm{min}]}\end{array}$ & $\begin{array}{l}\text { Pulm. Flow } \\
\text { [ml/min] }\end{array}$ & $\begin{array}{c}\text { MR } \\
\text { Qp/Qs } \\
\text { Before } \\
\text { Closure }\end{array}$ & $\begin{array}{c}\text { Oximetry } \\
\text { Qp/Qs } \\
\text { Before } \\
\text { Closure }\end{array}$ & $\begin{array}{c}\text { MR } \\
\text { Qp/Qs } \\
\text { After } \\
\text { Closure }\end{array}$ & $\begin{array}{c}\text { Balloon } \\
\text { Stretch } \\
\text { Diameter } \\
\text { MR [mm] }\end{array}$ & $\begin{array}{c}\text { Balloon } \\
\text { Stretch } \\
\text { Diameter } \\
\text { X-Ray } \\
\text { [mm] }\end{array}$ & $\begin{array}{c}\text { Balloon } \\
\text { Stretch } \\
\text { Diameter } \\
\text { Postmorte } \\
\text { m [mm] }\end{array}$ \\
\hline 1 & 88 & $90 / 50$ & 2386 & 3110 & 1.3 & 1.32 & 1.27 & 19 & 19 & 20 \\
\hline 2 & 91 & 136 / 99 & 3171 & 3780 & 1.19 & 1.18 & 0.99 & 16.6 & 16.7 & 14.9 \\
\hline 3 & 82 & $168 / 100$ & 4922 & 5217 & 1.05 & 1.05 & 0.92 & 15.6 & 16.7 & 16.9 \\
\hline 4 & 99 & 125 / 82 & 4189 & 4389 & 1.05 & 0.99 & 1.14 & 15.3 & 15.7 & 14.9 \\
\hline 5 & 87 & 130 / 80 & 4176 & 5933 & 1.42 & 1.26 & 1.04 & 20.2 & 17.9 & 21.1 \\
\hline 6 & 74 & 155 / 115 & 3726 & 4140 & 1.11 & 1 & 1.03 & 17.2 & 17.4 & 17.1 \\
\hline 7 & 82 & $140 / 100$ & 3867 & 5010 & 1.3 & 1.7 & 1.11 & 18.3 & 18.1 & 17.3 \\
\hline 8 & 82 & $110 / 72$ & 3565 & 4949 & 1.39 & 1.79 & 1.07 & 12.9 & 15.1 & 15.5 \\
\hline Mean & 86 & 132 / 87 & 3750 & 4566 & 1.23 & 1.29 & 1.07 & 16.9 & 17.1 & 17.2 \\
\hline St Dev. & \pm 8 & $\pm 25 / \pm 21$ & \pm 755 & \pm 893 & \pm 0.15 & \pm 0.31 & \pm 0.11 & \pm 2.3 & \pm 1.3 & \pm 2.3 \\
\hline
\end{tabular}

$\mathrm{HR}=$ heart rate; $\mathrm{BP}$ Syst./Diast.=systolic and diastolic femoral artery pressure; Aortic (Pulm.) Flow=flow determined by magnetic resonance flow measurements in the ascending aorta (pulmonary artery); MR Qp/Qs=ratio of pulmonary versus systemic blood flow determined by magnetic resonance flow measurements before and after closure of the atrial septal defect; Oximetry Qp/Qs=ratio of pulmonary versus systemic blood flow determined by oxygen saturation measurements; Balloon Stretch Diameter MR (X-Ray) [Postmortem]=diameter of waist of sizing balloon determined on magnetic resonance images (on $x$-ray images) [at postmortem]; Mean=mean values; St. Dev.= standard deviation 
In 2 animals, the baseline MR flow measurements of Qp/Qs ratios were 1.05, and they were therefore considered to have non-detectable shunts. Both animals had sizable ASDs by MR balloon sizing (15.6 and $15.3 \mathrm{~mm}$ ). Central injection of contrast media showed a tiny shunt of contrast media from the LA to the RA in one animal (Figure 3) and no shunting in the other.
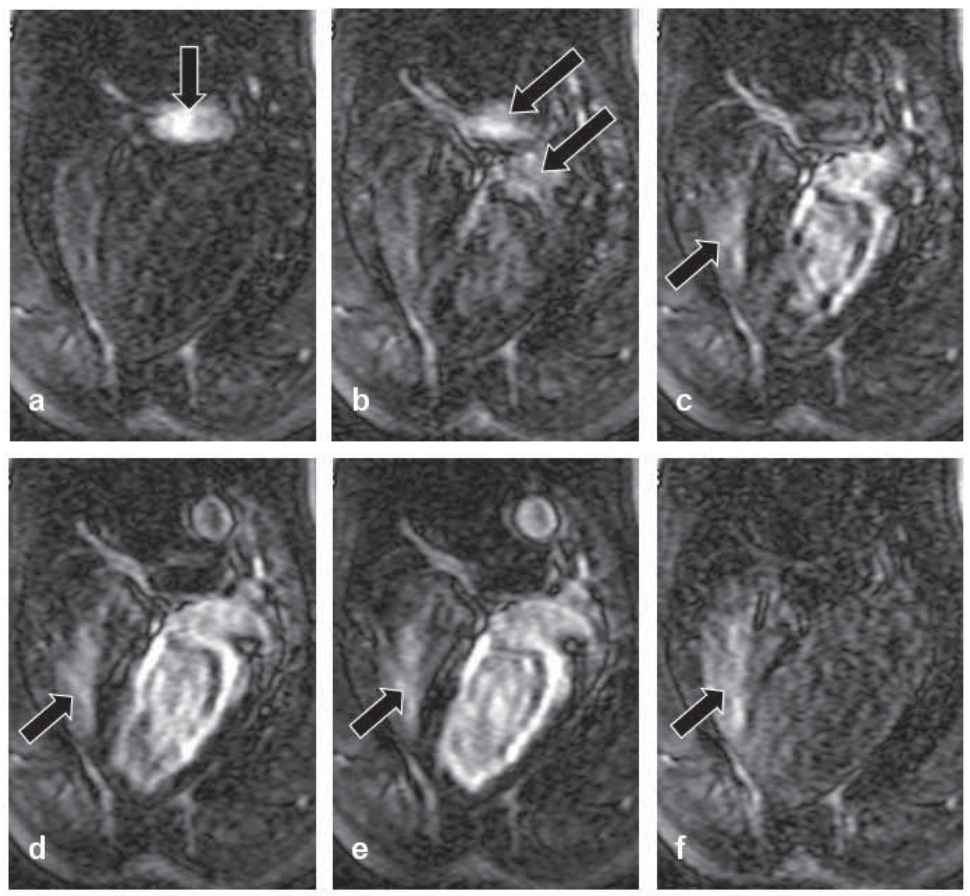

Figure 3: T1-weighted gradient echo images after injection of diluted Gd-DTPA-BMA into the main PA (cardiac 4-chamber view). Immediately after injection, contrast media enhancement of pulmonary veins is detected (a, arrow). Images b-e are consecutive images acquired 1 to $2.7 \mathrm{~s}$ after image a. Contrast media is seen in the pulmonary vein and LA ( $b$, arrows) and subsequently in the left ventricle (c-e). As a sign of an intracardiac left-to-right shunt, enhancement of the right ventricle is detected simultaneously with enhancement of left ventricle (c-e, arrows). Image f shows enhancement of the right ventricle during recirculation (12s after image a).

With MR balloon sizing, presence of ASD was demonstrated successfully in all animals (Figure 4). The stretched ASD diameter was similar on MR and $x$-ray measurements $(16.9 \pm 2.3 \mathrm{~mm}$ versus 17.1 $\pm 1.3 \mathrm{~mm} ; \mathrm{r}=0.91$; $\mathrm{SEE}=1.05, \mathrm{y}=1.64 \mathrm{x}-11.04)$. A Bland-Altman analysis revealed a value of $0.2 \pm 1.3 \mathrm{~mm}$ for absolute differences of waist diameter measurements. The postmortem balloon sizing was slightly larger at $17.2 \pm 2.3 \mathrm{~mm}$, but still had a good correlation with MR measurements $(r=0.82$; SEE = 1.42, $y=0.81 x+3.58$; absolute differences: $0.3 \pm 1.4 \mathrm{~mm}$ ). 


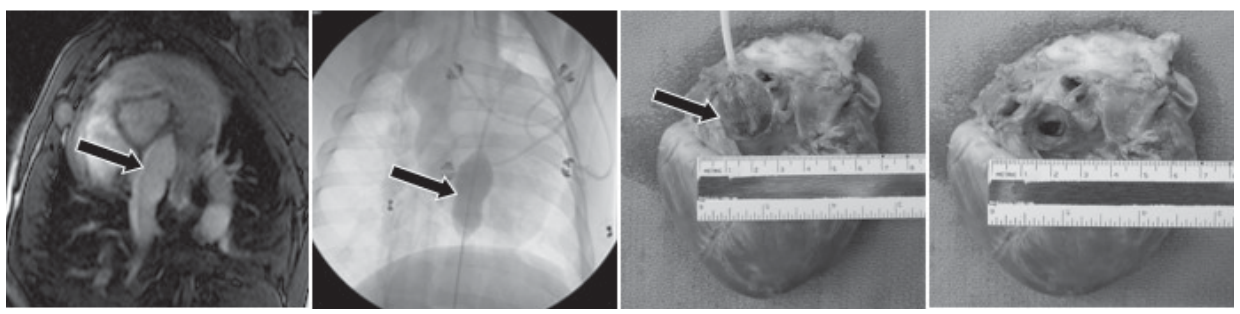

Figure 4: Balloon sizing of ASD with real-time MRI, x-ray fluoroscopy and postmortem: Note the waist diameter (arrows), representing the stretched ASD diameter, on images obtained with real-time MR fluoroscopy (left, inflated with Gd-DTPA-BMA), x-ray fluoroscopy (middle left, inflated with iodine contrast media) and postmortem (middle right, inflated with water).

\section{Transcatheter ASD closure with real-time MR guidance}

MR fluoroscopy in real-time was successfully used to guide transcatheter closure of ASDs in all of the animals. Both active and passive catheter tracking allowed correct sheath placement, although it was easier to monitor tip position with active tracking (Figure 5). Occasionally the delivery sheath and dilator were not seen well on MR images during passive tracking. Thus, contrast media was required in the lumen to enhance visibility. High resolution anatomical images and T1-weighted gradient echo images sensitive to Gd-DTPA-BMA enhancement were superimposed on real-time images to steer the catheter and sheath across the defect into LA. Time for transcatheter closure was 15-50 min, which decreased with increasing operator experience.

The use of commercially available to assemble a device delivery system enabled us to achieve device placement, recapture, and release similar to those obtained with factory-designed delivery systems. Due to the susceptibility effect, the loaded device inside the sheath was easily detected when it was advanced in the lumen towards the tip of the delivery sheath; however, because of the delivery system used, the effect was limited and did not obscure anatomical detail. Unfolding of the first disk in a balloon shape and subsequent umbrella shape outside the sheath was clearly visible (Figure 6), as was confirmation of its position against the septum. Release of the proximal disk was also clearly visible. Confirmation of septum in between the discs required the device to be advanced against the septum towards the LA until a slight resistance was felt, followed by retraction. This was necessary because the septum was not continuously visible, whereas it was always possible to watch the corresponding heart movement on real-time MRI during the "wiggle" procedure. Artifacts from the device were small enough to allow assessment of anatomy after placement, but large enough to allow imaging of the disks itself. An IVC-LA-RA image plane (Figure 6, lower right) and an RVRA-LA view (Figure 6, lower left) were considered the most important image planes for guiding the closure procedure, but rapid switching between different imaging planes was easy and allowed a comprehensive understanding of the current device position and its relationship to the surrounding anatomical structures. 

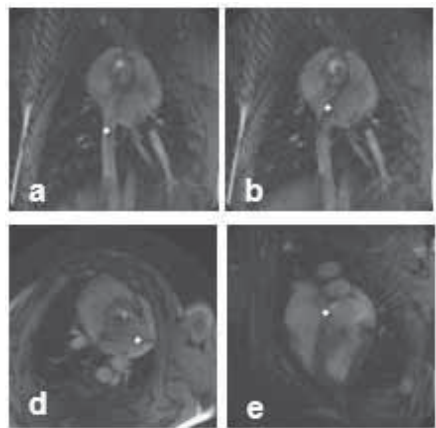
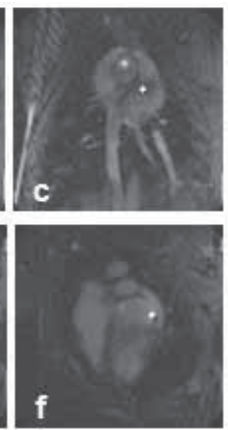
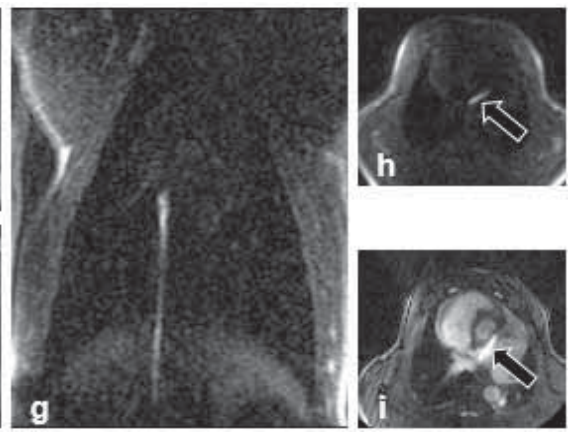

Figure 5: Transseptal sheath placement with active (a-f) and passive (g-i) catheter tracking using real-time steady state free precession MRI. Active tracking: The tip of a prototype active tracking catheter is detected as a blinking cross superimposed on real-time MR images. Employing an IVC-RA image plane, the catheter is steered from the IVC into the RA, and transseptally into the LA (a-c). Evaluation with additional image planes (RA-LA view, d; cardiac 4-chamber view, e, f) was important to confirm tip position. After the catheter tip had been steered into the LA, a guidewire was placed, over which the delivery sheath was subsequently advanced. Passive tracking: Using real-time MRI, the delivery sheath with dilator was steered directly across the septum without the help of an intravascular coil. Due to a small image void on MR images, the sheath lumen was filled with Gd-DTPA-BMA, and the tip position (arrows) was confirmed by both real-time (i) and T1-weighted gradient-echo imaging $(g, h)$. The contrast media filled sheath is seen in the IVC on an IVC-RA image plane $(\mathrm{g})$, and transseptally on an RA-LA image plane $(\mathrm{h}, \mathrm{i})$.
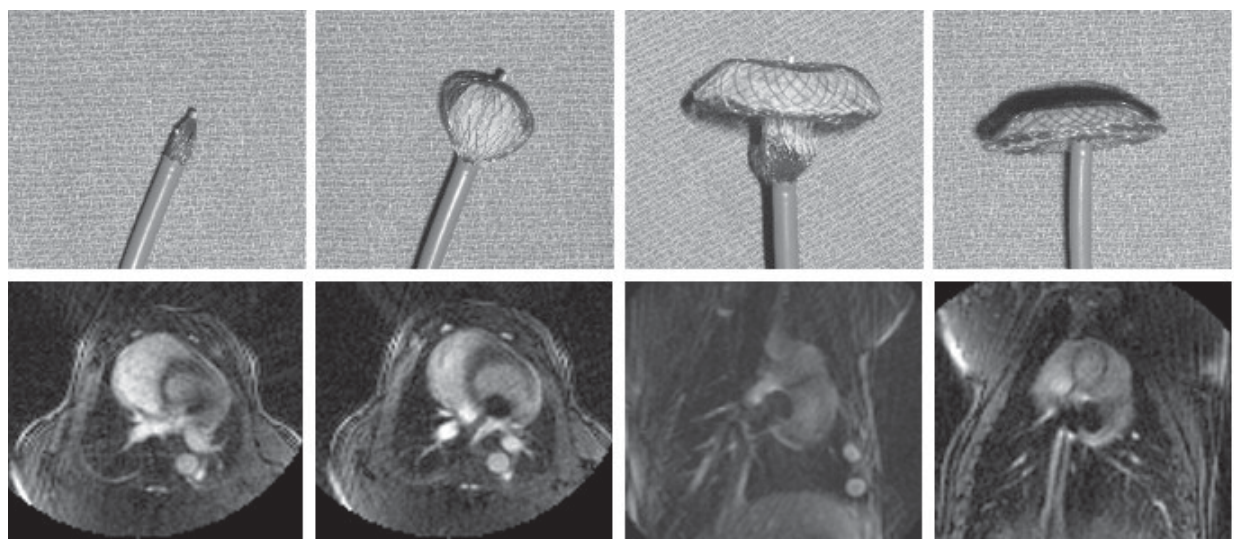

Figure 6: Simulation of the deployment of septal occluder device in vitro (upper row), and corresponding realtime MR images in vivo (bottom row): The device was easily detected as a signal void on MR fluoroscopic images. The closure device was advanced along the inside of the delivery sheath until the folded first disk appeared at the tip of the sheath in the left atrium (left image). The disk was then further advanced until it developed a balloon-like shape (second image from left). After it was further advanced, the first disk developed an umbrella-like shape in the LA while the second disk was about to be released at the level of the RA (second image from right). Finally, both disks were unfolded (right image). 


\section{Postmortem findings}

Visualization of the device in the septum at postmortem examination showed correct placement in all animals. No signs of hematoma, pericardial effusion, unintended perforation or other catheter-related injuries were observed.

\section{Discussion}

Assessment of the size and shunt across an ASD before and after transcatheter closure using commercially available materials was accomplished with MRI alone. Additionally, it was demonstrated that MR can accurately detect small intracardiac shunts (Qp/Qs $<1.5)$ under well defined physiologic conditions, with excellent correlation with saturation flow calculations. Since defect size correlated poorly with shunt size, the balloon stretch diameter was the most reliable measurement for documenting ASD size, and this was equally accurate for MR and $\mathrm{x}$-ray fluoroscopy.

Oximetry, indicator dilution, and radionuclide scintigraphy have been evaluated for the detection of left-to-right shunts. Oximetry is considered insensitive for detecting small shunts, and its estimate of shunt magnitude may be imprecise for large shunts. ${ }^{15,16}$ The sensitivity of the indocyanine green injection technique is only modestly better than oximetry for detecting shunts with smaller volumes, ${ }^{17}$ and is no longer used clinically. Radionuclide techniques allow the detection of shunts with a Qp/Qs ratio of as small as $1.2{ }^{16}$ but require the injection of a radioactive substance. Previous studies have employed MRI to quantify shunts in ASDs, with mixed success. Holmvang et al. measured the region of signal enhancement on phase contrast cine MR images to determine the ASD size in patients. ${ }^{10}$ Good correlation among x-ray balloon sizing, surgical templates, and MR imaging was found. However, that study involved defects with large shunts. Hundley et al. used MR flow measurements in the aorta and main PA to determine the Qp/Qs ratio for left to right shunting in patients with intracardiac shunts. ${ }^{9}$ However, patients with small shunt $(\mathrm{Qp} / \mathrm{Q} s<1.5)$ could not be discriminated from patients without ASDs. Beerbaum et al. found that direct shunt assessment using phase contrast MRI was not accurate in children with ASDs. ${ }^{11}$ Qp/Qs ratios obtained from flow measurements were more accurate. However, all of the patients in that study had a ratio of $>1.5$. Rickers et al measured right atrial and ventricular volumes and reported a significant volume reduction after defect closure. ${ }^{13}$ However, the reported Qp/Qs ratio of 1.7 before closure obtained from blood gas measurements was considerably higher than in our model. The Qp/Qs findings of a small shunt volume $(<1.5)$ prior to closure in the current study are in agreement with oximetric findings and previous results from swine subjected to acute ASDs. ${ }^{18}$ The significant reduction, but not absence, of the shunt after device closure due to a small residual shunt through the device fabric is in keeping with what has been reported in humans. ${ }^{19}$ Our study shows that in an acute 
model of ASD, accurate measurement of small left-to-right atrial shunts can be obtained with the use of velocity-encoded cine MR flow measurements. The difference between repeated MR flow measurements is approximately $5 \%$, which is equal to or better than that obtained with invasive oximetry. ${ }^{11,20}$

It may be difficult to measure the size of the ASD by MRI because of poor visualization of the defect edge if the atrial wall is thin $(<3 \mathrm{~mm})$. Similar to the current experience with angiographic ASD evaluation, this limitation was easily overcome with MR visualization of the defect during the Gd-DTPA-BMA filled balloon stretch diameter measurement. In the current study, ASDs were created with an $18 \mathrm{~mm}$ dilating balloon. This corresponded well with the measured stretched ASD diameter of approximately $17 \mathrm{~mm}$ with MR and x-ray fluoroscopy, as well as postmortem stretch diameter measurements. We found that Gd-DTPA-BMA balloon sizing was optimal for detecting and sizing the ASDs in all of the animals, and can be reliably used to determine the proper closure device size for each individual. MRI appears to be equal to $x$-ray fluoroscopy in its ability to detect ASD size and shape, and to have a significant advantage in its ability to show surrounding anatomical details.

Transesophageal echocardiography is an established method to assess ASD size. The correlation coefficient range for ASD dimensions determined by transesophageal echocardiography in comparison with surgical or balloon measurements was $r=0.73$ to $r=0.92 .^{21,22}$ Like MRI, transesophageal echocardiography can be used to guide transcatheter ASD closure. ${ }^{23}$ Even small or intermittent shunts can be detected by colorflow mapping or contrast media leakage. Until now, transesophageal echocardiography has been superior to MRI for defining the atrial septum. Thus, the value of MRI for ASD assessment and closure guidance in comparison with echocardiography remains to be assessed.

The time required for transcatheter closure of ASDs with MR guidance was 15-50 minutes. This represented a learning curve of the personnel involved, and became shorter with increasing operator experience. The time required for a closure procedure is related to the size and complexity of the defect. Due to technical improvements in closure systems and their handling optimized for $x$-ray guidance, the time required for ASD closures under $x$-ray fluoroscopy at our institution is less than $10 \mathrm{~min}$. However, closing large defects may require more time, in part because of the lack of good visualization of surrounding soft tissue and atrial walls, even with additional echocardiographic guidance.

Two recent studies have demonstrated the potential of real-time MRI for guiding transcatheter ASD closure; however, both studies required special experimental equipment to minimize artifacts during device deployment to allow adequate visualization. Instead of developing a custom-made prototype of a closure device or delivery system, ${ }^{12,13}$ we used a delivery system that consisted of commercially available materials and devices approved for cardiac catherization under x-ray fluoroscopy. In addition, a standard sizing balloon catheter was employed to size the ASDs. We were able to suc- 
cessfully perform ASD sizing and closure solely under MR guidance using commercially available materials, with no significant MR artifact. Catheter manipulation and sheath placement were effectively guided by both active and passive tracking under MR visualization. All aspects of device delivery, release, and post release assessment were accurately monitored with MR fluoroscopy, with the added benefit of excellent visualization of surrounding structures and their relationship to the implant. Under real-time MR guidance, a poorly positioned device could be easily retracted back into the delivery sheath as long as it was still connected to the snare wire. The design of the in-housemade delivery system would not allow us to recapture the device once it was released from the snare wire, due to small and inconsistent imaging artifacts with the snare out of the sheath. The employment of commercially available materials and closure devices indicates that small modifications of existing materials are sufficient to provide an MR compatible ASD closure system for animal studies. However, radiofrequency-induced heating and electrical conductors are important safety issues during MR-guided catheterization, and the safety of our system (which contains a snare wire) is not known. No perceptible heating was recognized, and no sparking was observed. However, no systematic assessment of safety was performed, and further studies are needed to address this issue. A major barrier to implementation of transcatheter closure in patients is currently the uncertainty about the safety of guidewires and device delivery systems in the MR scanner.

In conclusion, morphological and functional assessments and transcatheter closure of ASDs with small shunts using MRI as a single imaging tool were successfully performed. Gd-DTPA-BMA enhanced balloon sizing accurately detected and sized defects. MR flow measurement of small ASD shunt volumes was accurate and reliable. Both active and passive catheter tracking were employed to guide the catheter. Commercially available materials were used effectively in the MR scanner to close ASDs in animals. Additional safety studies are needed before MR-guided ASD closure can be introduced into clinical practice. Diagnostic evaluation and closure of ASDs with MRI could satisfy the imperative to avoid $\mathrm{x}$-ray exposure of children with congenital heart disease, with the added advantage that improved functional and anatomic information could be obtained.

\section{References}

1 Moss AJ, Adams FH. Heart disease in infants, children, and adolescents including the fetus and young. 5th ed. Baltimore, Md: Williams \& Wilkins. 1995

2 Campbell M. Natural history of atrial septal defect. Br Heart J 1970; 32: 820-826.

3 Oho S, Ishizawa A, Akagi T, Dodo H, Kato H. Transcatheter closure of atrial septal defects with the Amplatzer septal occluder--a Japanese clinical trial. Circ J 2002; 66: 791-794.

4 Dhillon $\mathrm{R}$, Josen $\mathrm{M}$, Henein $\mathrm{M}$, Redington A. Transcatheter closure of atrial septal defect preserves right ventricular function. Heart 2002; 87: 461-465. 
5 Modan B, Keinan L, Blumstein T, Sadetzki S. Cancer following cardiac catheterization in childhood. Int J Epidemiol 2000; 29: 424-428.

6 Wu JR, Huang TY, Wu DK, Hsu PC, Weng PS. Radiation exposure of pediatric patients and physicians during cardiac catheterization and balloon pulmonary valvuloplasty. Am J Cardiol 1991; 68: 221-225.

7 Perisinakis K, Damilakis J, Theocharopoulos N, Manios E, Vardas P, Gourtsoyiannis N. Accurate assessment of patient effective radiation dose and associated detriment risk from radiofrequency catheter ablation procedures. Circulation 2001; 104: 58-62.

8 Brochu MC, Baril JF, Dore A, Juneau M, De Guise P, Mercier LA. Improvement in exercise capacity in asymptomatic and mildly symptomatic adults after atrial septal defect percutaneous closure. Circulation 2002; 106: 1821-1826.

9 Hundley WG, Li HF, Lange RA, et al. Assessment of left-to-right intracardiac shunting by velocity-encoded, phase-difference magnetic resonance imaging. A comparison with oximetric and indicator dilution techniques. Circulation 1995; 91: 2955-2960.

10 Holmvang G, Palacios IF, Vlahakes GJ, et al. Imaging and sizing of atrial septal defects by magnetic resonance. Circulation 1995; 92: 3473-3480.

11 Beerbaum P, Korperich H, Barth P, Esdorn H, Gieseke J, Meyer H. Noninvasive quantification of left-toright shunt in pediatric patients: phase-contrast cine magnetic resonance imaging compared with invasive oximetry. Circulation 2001; 103: 2476-2482.

12 Buecker A, Spuentrup E, Grabitz R, et al. Magnetic resonance-guided placement of atrial septal closure device in animal model of patent foramen ovale. Circulation 2002; 106: 511-515.

13 Rickers $\mathrm{C}$, Jerosch-Herold M, Hu X, et al. Magnetic resonance image-guided transcatheter closure of atrial septal defects. Circulation 2003; 107: 132-138.

14 Bland JM, Altman DG. Statistical methods for assessing agreement between two methods of clinical measurement. Lancet 1986; 1: 307-310.

15 Cigarroa RG, Lange RA, Hillis LD. Oximetric quantitation of intracardiac left-to-right shunting: limitations of the Qp/Qs ratio. Am J Cardiol 1989; 64: 246-247.

16 Boehrer JD, Lange RA, Willard JE, Grayburn PA, Hillis LD. Advantages and limitations of methods to detect, localize, and quantitate intracardiac left-to-right shunting. Am Heart J 1992; 124: 448-455.

17 Carter SA, Bajec DF, Yannicelli E, Wood EH. Estimation of left-to-right shunt from arterial dilution curves. J Lab Clin Med 1960; 55: 77-88.

18 Kong H, Gu X, Titus JL, et al. Creation of an intra-atrial communication with a new Amplatzer shunt prosthesis: preliminary results in a swine model. Catheter Cardiovasc Interv 2002; 56: 267-271.

19 Tworetzky W, Moore P, Bekker JM, Bristow J, Black SM, Fineman JR. Pulmonary blood flow alters nitric oxide production in patients undergoing device closure of atrial septal defects. J Am Coll Cardiol 2000; 35: 463-467.

20 Powell AJ, Tsai-Goodman B, Prakash A, Greil GF, Geva T. Comparison between phase-velocity cine magnetic resonance imaging and invasive oximetry for quantification of atrial shunts. Am J Cardiol 2003; 91 : 1523-1525, A1529.

21 Morimoto K, Matsuzaki M, Tohma Y, et al. Diagnosis and quantitative evaluation of secundum-type atrial septal defect by transesophageal Doppler echocardiography. Am J Cardiol 1990; 66: 85-91.

22 Mehta RH, Helmcke F, Nanda NC, Hsiung M, Pacifico AD, Hsu TL. Transesophageal Doppler color flow mapping assessment of atrial septal defect. J Am Coll Cardiol 1990; 16: 1010-1016.

23 Ewert P, Berger F, Daehnert I, et al. Transcatheter closure of atrial septal defects without fluoroscopy: feasibility of a new method. Circulation 2000; 101: 847-849. 



\section{Magnetic resonance flow measurements in real-time: \\ Comparison with a standard gradient-echo technique}

C. Klein, S. Schalla, B. Schnackenburg, A. Bornstedt, E. Fleck, E. Nagel.

J Magn Reson Imaging. 2001 Sep;14(3):306-10. 


\section{Abstract}

Background: Ultrafast gradient systems and hybrid imaging sequences offer the opportunity to acquire phase contrast flow data in real-time.

Methods: In a 1.5-Tesla magnetic resonance (MR)-tomograph, peak velocity and volume flow were assessed in 36 large vessels (aorta) and 33 medium-sized vessels (carotid and iliac artery) using a real-time (segmented k-space turbo gradient-echo planar imaging sequence) in comparison with a gradient-echo technique.

Results: With the real-time technique, the matrix was reduced from 116 to 64, and temporal resolution changed from $30 \mathrm{msec}$ to $124 \mathrm{msec}$. Measurements of peak velocity correlated in large $(r=0.88)$ and medium-sized vessels $(r=0.81)$. Volume flow measurements correlated in large vessels $(r=0.87)$, however, a poor correlation $(r=0.64)$ was found in medium-sized vessels.

Conclusions: Scan time can be significantly reduced and images acquired without ECGtriggering. Flow volume can only be determined in large vessels with sufficient accuracy, mainly due to reduced spatial resolution in smaller vessels. 


\section{Introduction}

Magnetic resonance (MR) phase contrast velocity mapping offers a direct quantitative determination of flow dynamics. ${ }^{1}$ Several in vivo and in vitro studies have shown that MR flow measurements are highly accurate and reproducible for the evaluation of flow velocity and volume. ${ }^{2-8}$ The technique of phase contrast imaging has been described in detail in previous publications. ${ }^{1-3}$ In contrast to anatomical imaging, flow measurements require the acquisition of two data sets that are subtracted from each other. The remaining signal is proportional to flow velocity. With gradient-echo techniques, flow measurements covering the entire cardiac cycle (cine loop) can be acquired. However, these techniques are very time consuming and require several minutes for data acquisition. In addition, the results are averaged over several heart beats, which prohibits the assessment of short-term changes in flow patterns that occur, e.g., under pharmacological stress or during physiological maneuvers. ${ }^{9}$ Furthermore, long acquisition times make these sequences sensitive to motion, such as respiration, which may significantly reduce image quality and influence the results. ${ }^{10}$ To compensate for cardiac motion, standard gradient-echo flow measurements need to be electrocardiogram (ECG)triggered, causing a significant decay of image quality in patients with cardiac arrhythmias, such as atrial flutter or fibrillation, or multiple ventricular premature beats, because of large beat to beat variations. Faster gradients and new sequences allow for extremely fast data acquisition, for example, by the use of echo planar imaging, which

has been successfully used for phase-contrast flow measurements. ${ }^{9-15}$ The combination of turbo gradient-echo and echo planar imaging (16) allows one to acquire high quality images of the heart in real-time, which has been shown to allow an adequate assessment of end-diastolic and end-systolic volume. ${ }^{17}$ This sequence has now been modified for the determination of flow.

The aim of this study was to determine the accuracy of real-time flow measurements for the assessment of vessel size, flow velocity, and volume in large and mediumsized vessels in comparison to a standard gradient-echo technique.

\section{Materials and methods}

\section{Patients}

In 69 vessel segments (large vessels: ascending aorta $=10$ and descending aorta $=26$, medium-sized vessels: common carotid artery $=20$ and common iliac artery $=13$ ) from 47 patients (40 males, 7 females; age $58 \pm 11$ ), flow measurements were performed. Patients were included if they had an indication for MR angiography (e.g., aortic aneurysm, peripheral stenotic lesions), gave informed consent, had no contraindication for MR examination, and were in stable sinus rhythm. Patients with cardiac arrhythmias 
(atrial fibrillation, frequent premature beats) were excluded to ensure reliable flow measurements with the reference method (gradient-echo technique).

\section{Magnetic Resonance Imaging}

All patients were examined in the supine position with a 1.5-Tesla whole body MR scanner (ACS NT, Philips, the Netherlands, CPR 5) equipped with ultrafast gradients (23 $\mathrm{mT} / \mathrm{m}$ amplitude, $105 \mathrm{mT} / \mathrm{m} / \mathrm{msec}$ slew rate). For the common carotid artery, a commercially available neck coil was used; for all other vessels, a body coil was used. After a rapid survey with transverse, sagittal, and coronal views to localize the arteries, flow measurements perpendicular to the vessel were performed.

The field of view (FOV) of the flow sequences was adjusted for each patient individually, ranging from $200 \mathrm{~mm}$ to $350 \mathrm{~mm}$, to reduce foldover artifacts. The gradient-echo sequence was performed during free breathing using retrospective ECG gating. Twentyfour heart phases were acquired for each cardiac cycle, thus, temporal resolution varied depending on heart rate (e.g., $27.8 \mathrm{msec}$ with a heart rate of 90/minute and $35.8 \mathrm{msec}$ with a heart rate of 70/minute). The flow-compensated and flow-encoded measurements were performed at the same time after the R-wave in two consecutive heart beats. In contrast, in the real-time technique, the two measurements were performed consecutively during the same heart beat and 50 images were acquired during 6 seconds to ensure a data set of at least five or more cardiac cycles. FOV, slice thickness and encoded velocity were identical for both scans. To improve temporal resolution of the real-time sequence, the scan matrix was reduced, resulting in a lower spatial resolution. The details of the sequence are summarized in Table 1.

\section{Data Analysis}

Regions of interest (ROIs) were drawn manually on the anatomical image, then copied to the flow-encoded image. Care was taken to include the whole vessel lumen but to exclude the extravascular tissue. This process was repeated for each cardiac phase of the standard and for all images of the real-time technique. The vessel diameter was determined in the end-diastolic images. The first image after the R-wave in the standard technique, and the last image before flow acceleration in the real-time technique were defined as end-diastolic. Peak velocity ( $\mathrm{cm} / \mathrm{second}$ ) was determined from the fastest pixel in the ROI during systole. Volume flow per heart beat ( $\mathrm{mL} /$ heart beat) was calculated as the sum of the mean flow velocity multiplied by the area of the ROI of each phase. The mean value of four representative heart beats was calculated for the realtime scan. 
Table 1: Scan Parameters

\begin{tabular}{lll}
\hline & GRE & RT \\
\hline TR (msec) & 14 & 15.5 \\
TE (msec) & 3.5 & 6.8 \\
Flip angle & $15^{\circ}$ & $20^{\circ}$ \\
Field of view (mm) & $200-350$ & $200-350$ \\
Matrix ${ }^{a}$ & $115 \times 128$ & $64 \times 128$ \\
Slice thickness (mm) & 8 & 8 \\
NSA & 2 & 1 \\
Temporal resolution (msec) & Heart rate dependent & 124 \\
Encoded velocity (cm/second) & 200 & 200 \\
Echo planar imaging factor & - & 9 \\
\hline
\end{tabular}

GRE, gradient echo; RT, real-time.

a) All raw data were filtered and zero-filled to 256 .

\section{Statistics}

The mean and one standard deviation are given for all continuous data. Comparisons between different techniques were made using a linear regressions analysis. The paired t-test was used and a value of $P<0.05$ was considered significant. The mean relative difference of the two different methods was calculated by dividing the absolute difference by the mean of the two measurements. ${ }^{18}$

\section{Results}

In all vessels, adequate flow images could be obtained. The mean heart rate of the patients was $74 \pm 15 \mathrm{bpm}$, resulting in a mean scan duration of $138 \pm 29$ seconds for the gradient-echo sequence and $6.8 \pm 1.3$ phases per heart beat for the real-time technique (Fig. 1). An example of an anatomical and a flow-encoded image for the gradient-echo and the real-time technique of the aorta is shown in Figure 2. A typical flow curve achieved with the real-time technique can be seen on the left side of Figure 4 . On the ride side of Figure 4, a flow curve of the same patient performing a valsalva maneuver can be appreciated. 

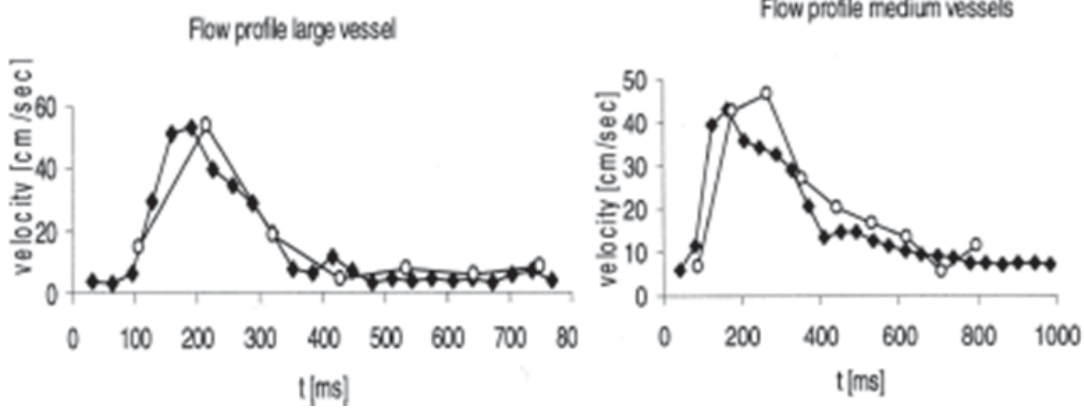

Figure 1: Example of a typical flow profile of large vessels (descending aorta) and medium-sized vessels (carotid artery) acquired with the real-time (open circles) and the standard gradientecho technique (closed rhombi). Note the similarity between the different acquisition techniques despite the better temporal resolution of the conventional technique.
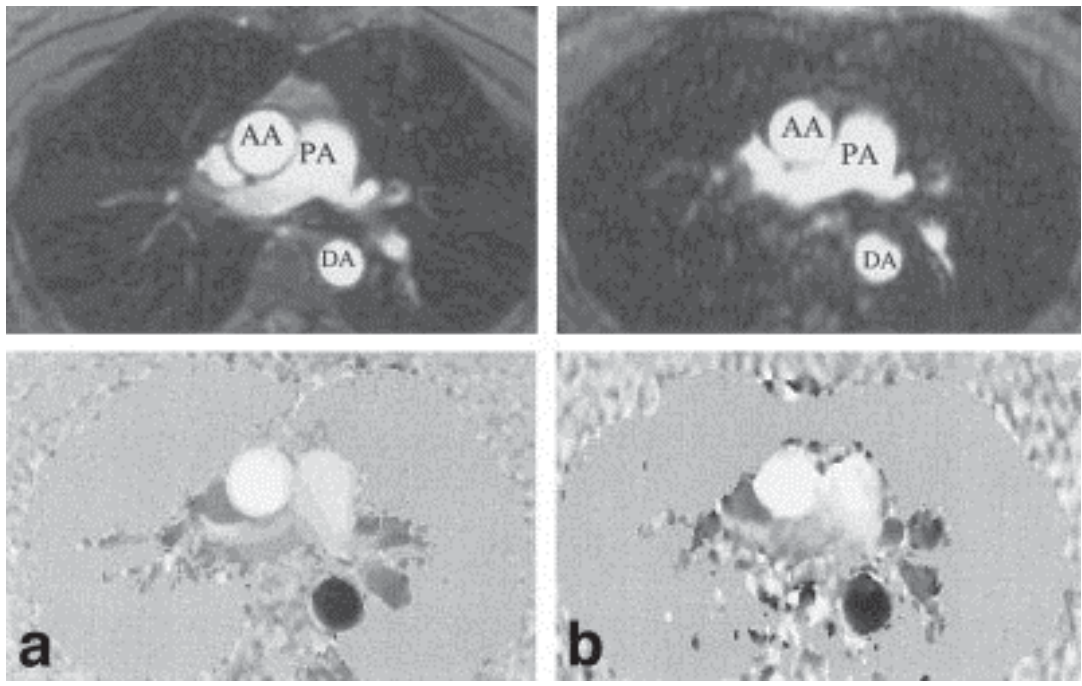

Figure 2a:. Midsystolic magnitude (top) and flow-encoded image (bottom) of a transverse slice through the thorax at the bifurcation of the pulmonary artery (PA) in conventional cine phasecontrast technique. The bright signal in the ascending aorta (AA) represents cranial flow and the dark signal in the descending aorta (DA) represents caudal flow. 2 b: Identical view as in Fig. a using the real-time technique. Even though spatial resolution is decreased and blurring is increased with the real-time technique, the flow signal can be clearly seen. 
a.)

Peak velocity of lange vervels

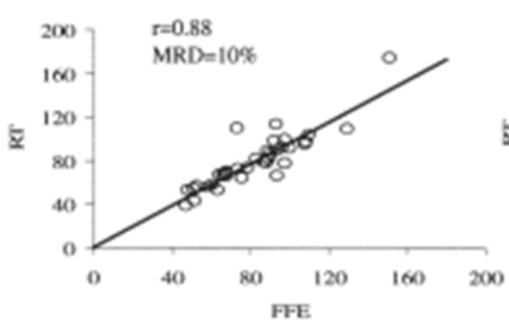

c.)

Peak velocity of medium sised vessels

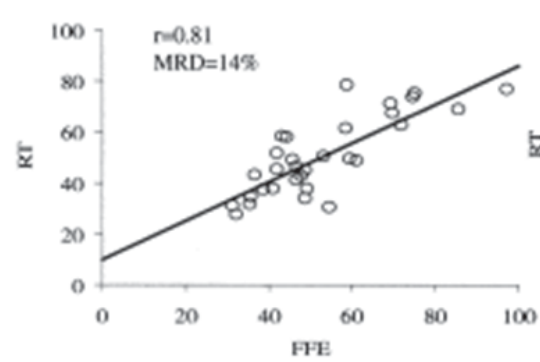

b.)

Volume flow of targe vessels

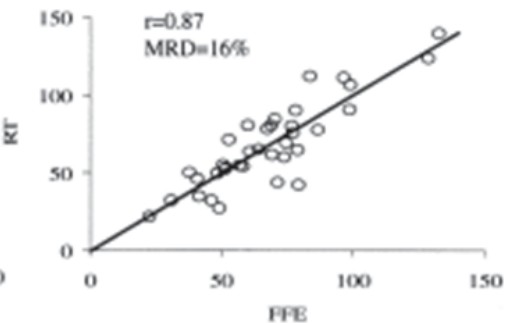

d.)

Volume flow of medium sized vessek

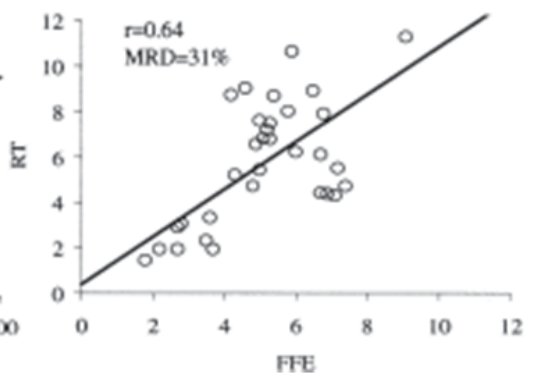

Figure 3: Linear regression analysis for peak velocity (a and c) and volume flow (b and d) measurements with the new real-time and a standard gradient-echo technique in large ( $a$ and $b$ ) and medium-sized vessels ( $c$ and d). FFE = gradient-echo technique; RT = real-time technique; $r$ = correlation coefficient; $\mathrm{MRD}=$ mean relative difference.
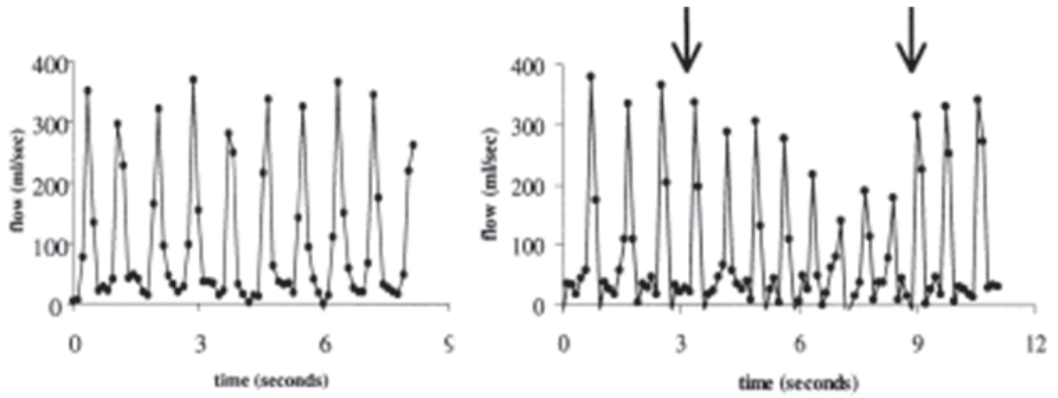

Figure 4: Typical real-time flow measurements in the ascending aorta. On the left of the figure is a patient holding his breath. On the right of the figure is the same patient performing a valsalva maneuver. Note the decrease in volume flow (arrows = begin and end of the valsalva maneuver). 
With the standard sequence, the average diameter of the large vessels was $30.8 \pm 6.41$ $\mathrm{mm}$ with a minimum of $22.0 \mathrm{~mm}$ and a maximum of $54.3 \mathrm{~mm}$; with the real-time sequence, the results were $30.9 \pm 6.4 \mathrm{~mm}$ with a minimum of $21.5 \mathrm{~mm}$ and maximum of $54.3 \mathrm{~mm}(P=0.32)$. There was a very good correlation $(r=0.97)$, with a slope of 0.97 and a point of interception at 1.1. The relative difference between the diameter measurements of the two techniques was $4 \%$. Thus, the number of cross sectional pixels was $130 \pm 60$ for the gradient-echo and $72 \pm 34$ for the real-time technique. In the mediumsized vessels, the diameter was $9.3 \pm 1.8 \mathrm{~mm}$ with a minimum of $6.5 \mathrm{~mm}$ and a maximum of $11.6 \mathrm{~mm}$ for the gradient-echo sequence and $9.4 \pm 1.4 \mathrm{~mm}$ with a minimum of $6.3 \mathrm{~mm}$ and a maximum of $12.8 \mathrm{~mm}$ for the real-time sequence $(P=0.67)$. There was a good correlation $(r=0.71)$, with a slope of 0.89 and a point of interception at 1.1 . The relative difference between the two measurements was $16 \%$. Therefore, the number of cross sectional pixels was $11 \pm 3$ for the gradient-echo and $6 \pm 2$ for the real-time technique.

Real-time peak flow velocity measurements correlated well with the conventional cine phase-contrast data in large and medium-sized vessels with a correlation coefficient of 0.88 and $0.81(P=0.13$ and 0.09 ), a slope of 0.96 and 0.86 , and a point of interception at 0.96 and 9.77, respectively. In large vessels, a mean relative difference of $10 \%$ was found; in medium-sized vessels, a mean relative difference of $14 \%$ was found. For volume flow, a correlation of $0.87(P=0.44)$ with a slope of 1.00 , a point of interception at -0.40 , and mean relative difference between the standard and the real-time technique of $16 \%$ was found in large vessels. In contrast, in medium-sized vessels, only a weak correlation of $0.64(P=0.03)$ with a slope of 1.08 , a point of interception at 0.39 , and a mean relative difference of $31 \%$, was found (Fig. 3). No systematic over- or underestimation could be observed. Mean values for flow volume and peak velocity with the standard deviation and range in large and medium-sized vessels are shown in Table 2.

Table 2: Results of the Flow Measurements

\begin{tabular}{llllll}
\hline & GRE & RT & $r$ & $P$ & Mean relative difference \\
\hline Large vessels & & & & & \\
VOL & $67.8 \pm 24.0$ & $67.5 \pm 27.5$ & 0.87 & 0.44 & $16 \%$ \\
(Range) & $(22.7-132.7)$ & $(21.5-139.5)$ & & & \\
VEL & $82.2 \pm 23.2$ & $80.0 \pm 25.2$ & 0.88 & 0.13 & $10 \%$ \\
(Range) & $(47.0-150.9)$ & $(39.2-174.6)$ & & & \\
Medium sized vessels & & & & \\
VOL & $5.2 \pm 1.7$ & $6.0 \pm 2.9$ & 0.64 & 0.02 & $31 \%$ \\
(Range) & $(1.8-9.1)$ & $(1.44-13.0)$ & & & \\
VEL & $52.2 \pm 16.0$ & $49.9 \pm 15.1$ & 0.81 & 0.09 & $14 \%$ \\
(Range) & $(31.3-97.1)$ & $(27.6-78.5)$ & & & \\
\hline
\end{tabular}

Mean, standard deviation, range correlation, significance and mean relative difference of flow volume (VOL) in $\mathrm{mL} /$ heartbeat and peak velocity (VEL) in $\mathrm{cm} /$ second for the gradient echo (GRE) and the real-time technique (RT) in large and medium sized vessels 


\section{Discussion}

With the new ultrafast real-time technique, it is possible to acquire high quality modulus and phase contrast images without ECG-triggering in the thoracic aorta and the common carotid and iliac arteries. Image quality of the modulus images is sufficient to evaluate size and shape throughout the cardiac cycle in normal and pathologically altered vessels. In large vessels, the determination of peak flow velocity and volume flow is possible in real-time. In medium-sized vessels, only peak flow velocity can be determined with sufficient accuracy, whereas a relative error of $31 \%$ for the determination of volume flow was found between the two techniques. Thus, real-time imaging is a valid alternative to conventional MR imaging and flow measurement techniques for the determination of flow velocity in large and medium-sized vessels and flow volume in large vessels. This new approach has several advantages in comparison to conventional MR flow measurements. Scan time can be reduced from more than 2 minutes to 6 seconds, and ECG-triggering is not required for these measurements, which improves patient comfort and reduces costs. In addition, patients can be rapidly screened for pathological flow patterns, which then allows several other examinations, such as perfusion or visualization of anatomy, to be performed at the same time. In contrast to conventional MR techniques, real-time imaging can be used in patients with atrial fibrillation or frequent premature ventricular beats, as image quality is independent of the underlying cardiac rhythm. Another potential advantage is the evaluation of flow during physiologic maneuvers, such as valsalva (Fig. 4) or during physical or pharmacological stress. In combination with interactive planning tools, this technique allows for an adaptation of the imaging plane during scanning. ${ }^{19}$

However, several limitations currently have to be accepted for the real-time technique. Temporal and spatial resolution are reduced in comparison to the standard techniques, which may introduce significant errors into phase-contrast flow measurements. ${ }^{10,20-22}$ Spatial resolution is especially important for the determination of flow volume because of the strong influence of partial volume effects from pixels that are partially placed within and partially placed outside the vessel or on the vessel wall. The smaller the vessel, the larger the relative number of pixels influenced by partial volume effects compared to the number of pixels fully in the vessel and, thus, the larger this influence on the result. ${ }^{20,22}$ To minimize partial volume effects, zero filling of the raw data by a factor of two was used in both techniques. ${ }^{23,24}$ This allows the utilization of the information contained in the raw data set to a greater extent. In large vessels with a mean diameter of $31 \mathrm{~mm}$, the number of cross sectional pixels is approximately 130 with the standard and 72 with the real-time technique. In medium-sized vessels with a mean diameter of $9.3 \mathrm{~mm}$, the number of cross sectional pixels is approximately 11 with the standard and 6 with the real-time technique, using the spatial resolution applied in the current study. This small number of pixels may explain the significant error of the volume flow measurements in medium-sized vessels. In contrast, in the aorta, the spa- 
tial resolution of the real-time sequence was sufficient and yielded results that differed only minimally from the reference standard. Interestingly, the correlation of the mean diameter between the standard and the real-time technique was lower for the mediumsized than for the large vessels (0.71 versus 0.97 ). Thus, errors in the determination of the vessel border in the real-time technique are an additional source of error for the measurement of flow volume. For the determination of the vessel diameter, the first image after the R-wave (standard technique) and the last before flow acceleration (realtime) was taken for the measurement. Both time points do not exactly represent the end-diastolic phase and may influence vessel diameter. For the determination of peak velocity, the fastest pixel within the vessel is used for the measurements and partial volume effects from stationary tissue play only a minor role. As spatial resolution was sufficient to ensure numerous pixels to be completely within the vessel, even in medium-sized vessels, only a small error was found for peak flow measurements in both large and medium-sized vessels. Possible improvements may be achieved by employing newly introduced techniques designed to reduce acquisition time by using the spatial sensitivity profiles of multiple coil elements (SMASH/SENSE) $)^{25,26}$ and can also be used to acquire a higher spatial resolution within the same measurement time. A different future approach may be the determination of flow by spiral scans, which has recently been shown to be possible without the subtraction of two data sets. ${ }^{27}$

To reduce measurement time, the combination of turbo gradient-echo with echo planar imaging, as used for the current study, has several advantages in comparison to pure echo planar imaging sequences. As data is collected over a free induction decay, and large echo planar imaging factors result in a long echo train, data lines collected beyond the tissue T2* decay will contain little signal. This makes the images prone to artifacts, especially around vessels, as T2* is short because of magnetic field inhomogenities of surrounding tissues. This limitation can be overcome by shortening the echo train and, thus, reducing the data lines acquired after each radiofrequency pulse. This is accomplished by the combination of a turbo gradient-echo technique and echo planar imaging. A shorter gradient-echo train reduces motion ${ }^{28,29}$ and field inhomogenityinduced spatial distortion, resulting in a more homogenous intravascular signal ${ }^{11}$ that enables easier delineation of the vascular border. A multishot ECG-triggered technique using a similar echo planar imaging factor and TE achieved excellent results compared to a standard gradient-echo technique in a flow phantom and the aorta. ${ }^{15}$ Nevertheless, with a temporal resolution of $124 \mathrm{msec}$ in the real-time compared with approximately $30 \mathrm{msec}$ in the standard technique, the point of maximal flow velocity during systole may not have been caught. Due to the averaging of velocities during data acquisition, one would suspect a systematic underestimation of peak velocity. This, however, was not the case in our experiment.

A limitation for the validation of the new real-time technique is the lack of an optimal reference standard. Quantitative MR flow measurements with a gradient-echo technique have been shown to be highly accurate ${ }^{30-32}$ and were, thus, used for compari- 
son. Other noninvasive techniques have their known limitations, e.g., the Doppler flow technique is limited by influences of angulation and acquisition window and was therefore not used for comparison in this study. Although the real-time sequence does not average the data over several heart cycles and provides real-time acquisition of the data, temporal resolution has to be improved for actual real-time determination of flow.

\section{Conclusions}

Real-time flow measurements allow a reliable determination of peak flow velocity in large and medium-sized vessels and the determination of flow volume in large vessels. With the new technique, scan time can be significantly reduced, which is a further step towards the integrative examination of patients with MR imaging. The accurate assessment of volume flow in large vessels allows one to determine cardiac output continuously during various hemodynamic aspects. Further studies must evaluate the value of this technique in patients with cardiac arrhythmias and accuracy of flow measurements through cardiac valves.

\section{References}

1. Pelc NJ, Herfkens RJ, Shimakawa A, Enzmann DR. Phase contrast cine magnetic resonance imaging. Magn Reson Q 1991; 7: 229-254.

2. Evans AJ, Iwai F, Grist TA, et al. Magnetic resonance imaging of blood flow with a phase subtraction technique: in vitro and in vivo validation. Invest Radiol 1993; 28: 109-115.

3. Pelc LR, Pelc NJ, Rayhill SC, et al. Arterial and venous blood flow: noninvasive quantitation with MR imaging. Radiology 1992; 185: 809-812.

4. Kondo C, Caputo GR, Semelca R, Foster E, Shimakawa A, Higgins CB. Right and left ventricular stroke volume measurements with velocity-encoded cine MR imaging: in vitro and in vivo validation. AJR Am J Roentgenol 1991; 157: 9-16.

5. Burkard DJ, Johnson CD, Morton MJ, Wolf RL, Ehman RL. Volumetric flow rates in the portal venous system: measurements with cine phase-contrast MR imaging. AJR Am J Roentgenol 1993; 160: 11131118.

6. Mohiaddin RH, Wann SL, Underwood R, Firmin DN, Rees S, Longmore DB. Vena cava flow: assessment with cine MR velocity mapping. Radiology 1990; 177: 537-541.

7. Debatin JF, Strong JA, Sostman HD, et al. MR characterization of blood flow in native and grafted internal mammary arteries. J Magn Reson Imaging 1993; 3: 443-450.

8. Bendel P, Buonocore E, Bockisch A, Besozzi MC. Blood flow in the carotid arteries: quantification by using phase-sensitive MR imaging. AJR Am J Roentgenol 1989; 152: 1307-1310.

9. Gatehouse PD, Firmin DN, Collins S, Longmore DB. Real-time imaging by spiral scan phase velocity mapping. Magn Reson Med 1994; 31: 504-512.

10. Debatin JF, Ting $\mathrm{RH}$, Wegmüller $\mathrm{H}$, et al. Renal artery blood flow: quantification with phase contrast MR imaging with and without breath holding. Radiology 1994; 190: 371-378. 
11. McKinnon GC, Debatin JF, Wetter DR, von Schulthess G. Interleaved echo planar flow quantification. Magn Reson Med 1994; 32: 1-5.

12. McKinnon GC. Interleaved echo planar phase contrast angiography. Magn Reson Med 1994; 31: 682-685.

13. Firmin DN, Klipstein RH, Hounsfield GL, Paley MP, Longmore DB. Echo-planar high resolution flow velocity mapping. Magn Reson Med 1989; 12: 316-327.

14. Guilfoyle DN, Gibbs P, Ordidge RJ, Mansfield P. Real-time flow measurements using echo planar imaging. Magn Reson Med 1991; 18: 1-8.

15. Debatin JF, Leung DA, Wildermuth S, Botnar R, Felblinger J, McKinnon GC. Flow quantification with echoplanar phase contrast velocity mapping: in vitro and in vivo evaluation. J Magn Reson Imaging 1995; 5: 656-662.

16. McKinnon GC. Ultrafast interleaved gradient-echo-planar imaging on a standard scanner. Magn Reson Med 1993; 30: 609-616.

17. Nagel E, Schneider U, Schalla S, et al. Magnetic resonance real-time imaging for the evaluation of left ventricular function. J Cardiovasc Magn Reson 2000; 2: 7-14.

18. Bland JM, Altman DG. Statistical methods for assessing agreement between two methods of clinical measurement. Lancet 1986; 8: 307-310.

19. Yang $P C$, Kerr AB, Liu AC, et al. New real-time interactive cardiac magnetic resonance imaging system complements echocardiography. J Am Coll Cardiol 1998; 32: 2049-2056.

20. Hoffmann MB, Visser FC, van Rossum AC, Vink GQ, Sprenger M, Westerhof N. In vivo validation of magnetic resonance blood volume flow measurements with limited spatial resolution in small vessels. Magn Reson Med 1995; 33: 778-784.

21. Wolf RL, Ehman RL, Riederer SJ, Rossmann PJ. Analysis of systematic and random error in MR volumetric flow measurements. Magn Reson Med 1993; 30: 82-91.

22. Tang C, Blatter DD, Parker DL. Accuracy of phase-contrast flow measurements in the presence of partial volume effects. J Magn Reson Imaging 1993; 3: 377-385.

23. Parker DL, Yiping DP, Davis DL. The voxel sensitivity function in fourier transform imaging: application to magnetic resonance angiography. Magn Reson Med 1995; 33: 156-162.

24. Parker DL, Parker DJ, Blatter DL, Yiping DP, Goodrich KC. The effect of image resolution on vessel signal in high-resolution magnetic resonance angiography. J Magn Reson Imaging 1996; 6: 632-641.

25. Weiger $M$, Scheidegger MB, Prüssmann KP, Boesiger P. Cardiac real-time acquisition using coil sensitivity encoding. In: Proceedings of the 6th Annual Meeting of ISMRM, Sydney, Australia, 1998.

26. Sodickson DK, Manning WJ. Simultaneous acquisition of spatial harmonics (SMASH): fast imaging with radiofrequency coil arrays. Magn Reson Med 1997; 38: 591-603.

27. Man LC, Pauly JM, Ishimura DG, Macovski A. Nonsubtractive spiral phase contrast velocity imaging. Magn Reson Med 1999; 42: 704-713.

28. Duerk JL, Simonetti OP. Theoretical aspects of motion sensitivity and compensation in echo-planar imaging. J Magn Reson Imaging 1991; 1: 643-650.

29. Butts K, Riederer SJ. Analysis of flow effects in echo-planar imaging. J Magn Reson Imaging 1992; 2: 285293.

30. Firmin DN, Nayle GL, Kilner PJ, Longmore DB. The application of phase shifts in NMR for flow measurements. Magn Reson Med 1990; 14: 230-241.

31. Boesiger P, Maier SE, Kecheng L, Scheidegger MB, Meier D. Visualization and quantification of human blood flow by magnetic resonance imaging. J Biomech 1992; 25: 55-67.

32. Meier D, Maier S, Boesiger P. Quantitative flow measurements on phantoms and on blood vessels with MR. Magn Reson Med 1988; 8: 25-34. 
Real-time MR image acquisition during high-dose dobutamine hydrochloride stress for detecting left ventricular wall-motion abnormalities in patients with coronary arterial disease

S. Schalla, C. Klein, I. Paetsch, H. Lehmkuhl, A. Bornstedt, B. Schnackenburg, E. Fleck, E. Nagel.

Radiology. 2002 Sep;224(3):845-51. 


\section{Abstract}

Purpose: To compare the accuracy of real-time magnetic resonance (MR) imaging with that of standard echo-planar MR imaging for detecting myocardial wall-motion abnormalities at rest and during dobutamine hydrochloride-induced stress in patients with coronary arterial disease.

Materials and methods: In 22 patients with coronary arterial disease, left ventricular wall motion was examined at rest and during dobutamine hydrochloride stress, by using echo-planar MR imaging and a new technique with real-time segmented k-space turbo gradient-echo echo-planar MR imaging (repetition time, $16.5 \mathrm{msec}$; echo time, 6.8 msec). Wall-motion abnormalities were determined visually for each perfusion territory, and Cohen $\mathrm{k}$ coefficients were calculated for real-time imaging in comparison with echo-planar imaging. Coronary angiography was performed in all patients. Sensitivity and specificity for real-time and echo-planar imaging were calculated for detecting significant coronary arterial stenosis.

Results: $\mathrm{k}$ values for detecting wall-motion abnormalities at real-time imaging, in comparison with echo-planar MR imaging, were 0.97 at rest and 0.94 at maximum dobutamine hydrochloride stress. At comparison with those of angiography, the sensitivity and specificity for detecting significant coronary arterial stenosis were 88\% (14 of 16 patients) and $83 \%$ (five of six patients), respectively, for echo-planar imaging and 81\% (13 of 16 patients) and $83 \%$ (five of six patients), respectively, for real-time imaging.

Conclusions: Real-time MR imaging is possible under stress conditions and allows accurate detection of wall-motion abnormalities. 


\section{Introduction}

Noninvasive detection of coronary arterial disease (CAD) is a major challenge. With exercise echocardiography (ECG), only limited information about the localization and extent of CAD can be obtained, and sensitivity and specificity are low. ${ }^{1}$ At the time this article was written, dobutamine hydrochloride-induced stress ECG was one of the most widely used methods for detecting myocardial ischemia on the basis of visualization of wall-motion abnormalities (WMAs). However, this technique is limited by moderate image quality in $10 \%-15 \%$ of all examinations, ${ }^{2,3}$ although recent improvements in ECG techniques, such as harmonic imaging, might improve image quality, ${ }^{4,5}$ and the need for acoustic windows, which are defined by the anatomy of the patient.

Magnetic resonance (MR) imaging of the heart is highly accurate and reproducible for determining left ventricular volumes, function, and muscle mass with use of spinecho, ${ }^{6-9}$ gradient-echo, or echo-planar breath-hold MR imaging, with low interstudy variability. ${ }^{10-16}$ It has been shown that dobutamine hydrochloride stress MR imaging is superior to dobutamine hydrochloride stress ECG for noninvasive detection of CAD, especially in patients with moderate ECG image quality (ie, myocardial motion detectable in $\geq 13$ segments, but no clear endocardial border). ${ }^{17-19}$ However, even with turbo techniques, several limitations of MR imaging when compared with ECG remain because of image acquisition during several heartbeats. Image acquisition requires approximately 10-16 seconds and an additional 3-4 seconds for image reconstruction, which prohibits display and analysis or adaption of image planes in real-time. This limitation has been regarded as a potential safety problem of MR imaging during stress testing. ${ }^{20}$ In addition, image quality is reduced by cardiac arrhythmias or breathing motion and is thus performed during breath holding.

The development of high-performance gradient systems and optimized hybrid sequences that combine turbo gradient-echo and echo-planar MR imaging makes acquisition of complete cardiac images in real-time possible. ${ }^{21,22}$ This technique has several advantages when compared with conventional acquisition techniques because the complete data set is acquired during a single measurement interval and not during several heartbeats. Breath holding is not necessary to preserve image quality and, although ECG monitoring is important for the patient's safety, it is no longer required to trigger imaging.

In combination with interactive planning tools, real-time planning and adaption of imaging planes can be performed. ${ }^{23}$ However, in contrast with conventional MR imaging techniques, spatial resolution is reduced and a high number of echo-planar imaging readouts are used to reach adequate temporal resolution, which may lead to image distortion and reduce accuracy.

It has been reported that real-time image quality is sufficient for assessment of left ventricular function and may be superior to that obtained at ECG. ${ }^{23}$ Close correlation of real-time and conventional MR imaging for determining left ventricular volumes has 
been found. $^{22}$ If real-time imaging were sufficiently accurate to depict new WMAs, it could be used to monitor or even to examine patients during stress examinations.

The aim of the current study was to determine the accuracy of real-time MR imaging when compared with conventional echo-planar MR imaging for detecting WMAs at rest and during dobutamine hydrochloride stress.

\section{Materials and methods}

Patients

The study was approved by the institutional review committee of Humboldt University in Berlin. Patients who were scheduled to undergo coronary angiography, were known to have $C A D$, and had previously undergone percutaneous transluminal coronary angioplasty (with or without stent implantation) or bypass surgery consecutively underwent screening for study inclusion. For safety reasons, patients were excluded from the study if one or more of the following criteria were met: myocardial infarction in the past 4 weeks, unstable angina pectoris, known left main coronary arterial stenosis, New York Heart Association class III heart failure, class II valvular disease, dilated or obstructive cardiomyopathy, ejection fraction of less than 20\%, blood pressure higher than 220/110 $\mathrm{mm} \mathrm{Hg}$, or claustrophobia. Twenty-five consecutive patients were included after written informed consent was obtained, depending on the availability of the MR imager. At the time of imaging, two patients were excluded from the study because of previously unknown claustrophobia. In an additional patient, technical problems prohibited MR imaging, which resulted in a study population of 22 patients. The characteristics of the study group are listed in table 1. 
Table 1: Patient characteristics

\begin{tabular}{|c|c|}
\hline Parameter & Finding \\
\hline Number of patients & 22 (19 men, 3 women) \\
\hline Mean age [years] & $60.4 \pm 5.5$ \\
\hline Mean height $[\mathrm{cm}]$ & $172 \pm 8.4$ \\
\hline Mean weight [kg] & $79.3 \pm 12.3$ \\
\hline \multicolumn{2}{|l|}{ Medical history } \\
\hline Myocardial infarction & 18 \\
\hline Angioplasty ( \pm stent) & 20 \\
\hline Bypass surgery & 8 \\
\hline \multicolumn{2}{|l|}{ Coronary artery disease } \\
\hline One vessel & 2 \\
\hline Two vessels & 9 \\
\hline Three vessels & 11 \\
\hline \multicolumn{2}{|l|}{ Mean heart rate [beats per minute] } \\
\hline Resting & $70 \pm 8$ \\
\hline Age predicted & $136 \pm 5$ \\
\hline Maximum & $145 \pm 8$ \\
\hline \multicolumn{2}{|l|}{ Mean blood pressure [mmHg] } \\
\hline Resting systolic & $132 \pm 18$ \\
\hline Resting diastolic & $81 \pm 12$ \\
\hline Maximum systolic & $153 \pm 28$ \\
\hline Maximum diastolic & $76 \pm 13$ \\
\hline Mean maximum dobutamine dose $[\mu \mathrm{g} / \mathrm{kg}$ per minute] & $39 \pm 4.3$ \\
\hline Mean atropine dose [mg] & $0.31 \pm 0.27$ \\
\hline
\end{tabular}

\section{Stress Testing Protocol}

Dobutamine hydrochloride (Dobutamine $280 \mathrm{mg} / 50 \mathrm{~mL}$; Fresenius Deutschland, Bad Homburg, Germany) was infused intravenously over 3 minutes per dose at doses of 10, 20,30 , and $40 \mu \mathrm{g}$ per kilogram of body weight per minute and was stopped at whatever dose was being administered when $85 \%$ of the age-predicted heart rate was reached. The highest dose, however, was continued and supplemented with 0.25-mg fractions of atropinsulfate (Atropine $0.5 \mathrm{mg} / \mathrm{mL}$; Braun Melsungen, Melsungen, Germany) (maximal dose, $1 \mathrm{mg}$ ), administered intravenously as a slow bolus, if less than $85 \%$ of the agepredicted heart rate was achieved with the maximum dose of dobutamine hydrochloride and if stress test findings were negative. Esmolol hydrochloride ([5 mg/kg intravenously as a slow bolus] Brevibloc 100 mg/10 mL; Baxter Deutschland, Unterschleissheim, Germany) and glyceroltrinitrat (0.4 mg Nitrolingual spray; G. Pohl-Boskamp, Hohenlockstedt, Germany) were administered after the test when clinically indicated. Stress testing was discontinued at patient request or when the following occurred: new WMAs (detected by means of echo-planar images displayed later at the next stress (evel), chest discomfort indicative of progressive or severe angina, dyspnea, systolic 
blood pressure decrease of more than $40 \mathrm{~mm} \mathrm{Hg}$, arterial hypertension (blood pressure $\geq 240 / 120 \mathrm{~mm} \mathrm{Hg}$ ), severe arrhythmia, or other serious adverse effects. ECG rhythm, blood pressure, and symptoms were monitored continuously.

\section{MR Imaging}

Patients were examined in the supine position by using a 1.5-T whole-body MR imager (Gyroscan ACS-NT; Philips Medical Systems, Best, the Netherlands) with research software (Cardiac Patch CPR6) and rapid-gradient systems $(21 \mathrm{mT} / \mathrm{m}$ amplitude, 100 $\mathrm{mT} / \mathrm{m} / \mathrm{sec}$ slew rate). A dedicated five-element phased-array cardiac coil placed around the thorax of the patient was used for image acquisition. A small field of view was used to decrease acquisition time. Only the two anterior segments of the coil were applied for data acquisition to avoid aliasing with the small fields of view.

After two rapid surveys to determine the axis of the left ventricle, three short-axis sections were obtained by using as a reference standard a segmented k-space echoplanar imaging sequence routinely used at our institution for stress MR imaging. Images were acquired during end-expiratory breath holds of approximately 12-16 heartbeats for each section. ${ }^{24}$ The details of the sequence are shown in table 2 . Image acquisition was then repeated with use of a real-time technique, with identical section positions.

Table 2: Magnetic resonance imaging scan parameters. All measurements were performed with flow compensation.

\begin{tabular}{lll}
\hline & MR imaging technique & \\
\cline { 2 - 3 } Imaging parameter & Echo-planar & Real-time \\
\hline Echo time [ms] & 5.6 & 6.8 \\
Repetition time & 1 heartbeat & $16.5 \mathrm{~ms}$ \\
Flip angle & 30 & 20 \\
K-lines per shot & - & 36 \\
Echo-planar imaging-factor & 7 & 9 \\
Matrix* & $102 \times 128$ & $64 \times 128$ \\
Temporal resolution [ms] & 30 & 62 \\
Spatial resolution [mm] & $1.3 \times 2.6$ & $2.2 \times 4.4$ \\
Slice thickness [mm] & 8 & 8 \\
\hline
\end{tabular}

*Raw data were filtered and zero-filled to 256 points.

The real-time sequence consists of a hybrid segmented k-space turbo gradient-echo echo-planar imaging sequence, ${ }^{21-23}$ with a repetition time msec/echo time msec of 16.5/6.8, a temporal resolution of $62 \mathrm{msec}$ (16 images per second) and a spatial resolution of $2.2 \times 4.4 \mathrm{~mm}$ (Table 2). Thirty consecutive images were acquired to cover at least two complete cardiac cycles. Zero filling was applied to better use the information contained in the raw data ( $\mathrm{k}$ space) and to reduce partial volume effects and edgedetection artifacts. To ensure similar conditions and geometry when compared with the 
reference technique, real-time imaging was also performed during end expiration and with R-wave ECG triggering of acquisition of the first image at end-diastole, even though this was not required to preserve image quality. The imaging time for real-time multisection acquisition of short-axis views was a maximum of 6 seconds. To keep the duration of dobutamine hydrochloride infusion as short as possible, real-time images of identical geometry were obtained with the patient at rest, at a dose of $20 \mu \mathrm{g}$, and at the maximum dobutamine hydrochloride and atropine sulfate doses. Image acquisition started approximately 1 minute after the beginning of administration of a stress level dose. During an examination, echo-planar images of the middle short-axis section were displayed at the next dobutamine hydrochloride stress level for rapid review of new onsets of WMAs. Because of hardware restrictions, real-time images were reconstructed later. Echo-planar images were acquired at every stress level. The total time for an examination with use of both MR imaging techniques was 30-40 minutes. No postprocessing was performed; images were, however, analyzed later.

\section{Qualitative MR Image Analysis}

All images were displayed as continuous cine loops and assessed visually. Image quality, endocardial movement, and systolic wall thickening, which were used to compare images obtained at rest and at medium and peak stress, were evaluated independently off line by two experienced cardiologists (S.S., E.N.) (with 3 years of experience each) who were blinded to results obtained with any other technique. In cases of discrepancy, consensus was reached by means of joint image review. Image quality was assessed as diagnostic or nondiagnostic. It was considered diagnostic if all 16 segments could be visualized and interpreted with regard to wall motion.

Similar to the procedure used at routine stress ECG, the left ventricular myocardium was divided into 16 segments. Each of the 16 segments was individually assessed for wall motion at rest, during increasing dobutamine hydrochloride stress levels (for realtime imaging only at $20 \mu \mathrm{g}$ ) and at maximum stress. Segmental wall motion was graded as normal or abnormal. Similar to the procedure for stress ECG, segmental wall motion was subjectively considered abnormal if hypokinesia (reduction of endocardial motion and systolic wall thickening), akinesia (absence of endocardial motion and systolic wall thickening), or dyskinesia (paradoxic wall motion) was observed. Every segment was assigned to the perfusion territory of a specific coronary artery, as suggested by the American Society of Echocardiography (Figure 1). ${ }^{25}$ Results were determined for perfusion territories. Results of ischemia detection were determined for each patient. Results for each patient were considered positive and indicative of myocardial ischemia in a perfusion territory if new or worsening WMAs in one or more segments developed during dobutamine hydrochloride stress or if WMAs observed at rest that improved during low-dose stress deteriorated during peak stress. 


\section{Apical}

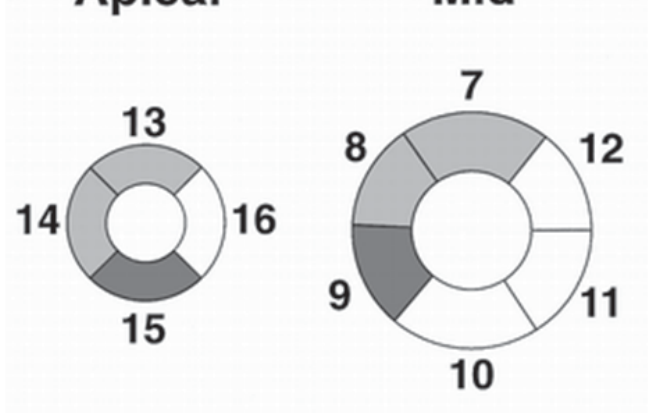

\section{$L A D \square R C A \quad \square L C X$}
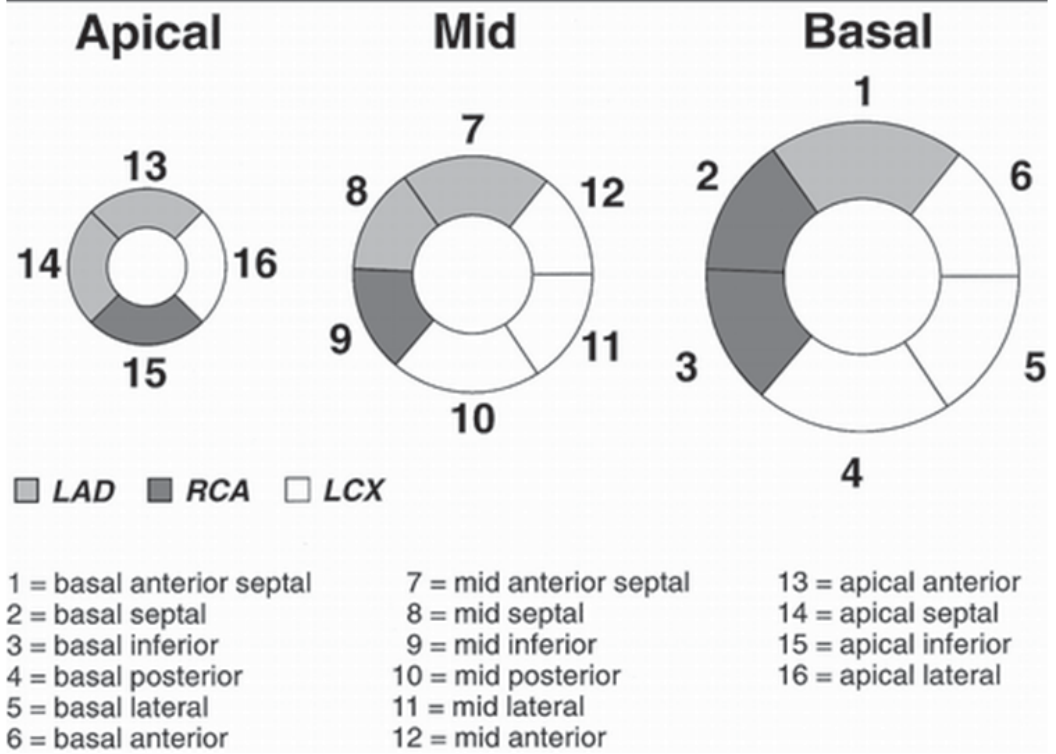

$$
\begin{aligned}
7 & =\text { mid anterior septal } \\
8 & =\text { mid septal } \\
9 & =\text { mid inferior } \\
10 & =\text { mid posterior } \\
11 & =\text { mid lateral } \\
12 & =\text { mid anterior }
\end{aligned}
$$

$$
\begin{aligned}
& 13=\text { apical anterior } \\
& 14=\text { apical septal } \\
& 15=\text { apical inferior } \\
& 16=\text { apical lateral }
\end{aligned}
$$

Figure 1: Diagram shows a segment model of the left ventricle: Short-axis view of the basal, middle, and apical left ventricular myocardium. The myocardium is divided into 16 segments that are assigned to perfusion territories of the coronary arteries. ${ }^{25,26} L A D=$ left anterior descending coronary artery, $L C X=$ left circumflex coronary artery, and $R C A=$ right coronary artery.

\section{Angiography}

Biplanar coronary angiography was performed after dobutamine hydrochloride stress MR imaging in all patients. Coronary arterial stenoses were imaged in multiple projections, minimizing overlapping of side branches and foreshortening of relevant coronary arterial stenoses.

Coronary angiograms were interpreted by the examiner and reviewed by the clinical conference chairman and, in cases of discrepancy, reviewed with a third reviewer (E.F.). Quantitative coronary angiography was performed in cases of doubt, when visual results could not clearly be assigned to a stenosis of $25 \%, 50 \%$, or $75 \%$ or greater (Quansad Quantitative Coronary Angiography postprocessing equipment; Arri, Munich, Germany). All three reviewers were experienced cardiologists (5-20 years experience) blinded to the results of noninvasive testing. Significant CAD was defined as an area reduction greater than $75 \%$ with respect to prestenotic segment areas in at least one major epicardial coronary artery or a major branch of one of these vessel distributions or coronary arterial bypass graft. 


\section{Data Analysis}

Sensitivity was calculated by using the following formula: Sensitivity = true-positive finding/(true-positive finding + false-negative finding); specificity = true-negative finding/(false-positive finding + true-negative finding).

As a measurement of agreement, Cohen k coefficients were calculated. The k coefficient equals 1 when there is complete agreement of two methods. When the observed agreement exceeds chance agreement, $\mathrm{k}$ is positive, with its magnitude reflecting strength of agreement. A value greater than 0.7 was considered satisfactory.

\section{Results}

In all patients, the age-predicted heart rate was reached during dobutamine hydrochloride stress, with a mean dose of $39.00 \pm 4.30 \mu \mathrm{g} / \mathrm{kg} / \mathrm{m}$ plus $0.31 \mathrm{mg} \pm 0.27$ atropine (Table 1). No major adverse effects of dobutamine hydrochloride were observed. Image quality was diagnostic for echo-planar and real-time imaging: Image quality was sufficient for qualitative analysis of wall motion with use of both techniques in all patients.

The numbers of perfusion territories with WMAs detected with echo-planar and real-time imaging at rest and maximum dobutamine hydrochloride stress are shown in table 3. With performance of the Cohen $\mathrm{k}$ test, values of 0.97 for the diagnosis of WMAs at rest and of 0.94 at maximum dobutamine hydrochloride stress were obtained for real-time imaging, when compared with echo-planar imaging.

Table 3: Perfusion territories with WMAs detected with echo-planar and real-time imaging at rest and maximum dobutamine stress

\begin{tabular}{lll}
\hline & EPI findings & \\
\cline { 2 - 3 } Variable and finding & Positive & Negative \\
\hline Rest & 25 & 1 \\
RTI findings positive & 0 & 40 \\
RTI findings negative & & \\
Stress & 29 & 1 \\
RTI findings positive & 1 & 35 \\
RTI findings neagtive & & \\
\hline
\end{tabular}

Note.-There were 66 perfusion territories. EPI = echo-planar imaging, RTI = real-time imaging.

With real-time imaging, new or worsening WMAs or a biphasic response to dobutamine hydrochloride, either of which was indicative of ischemia, was found in 96\% (22 of 23) of all positive results with use of the standard technique (Cohen $\mathrm{k}$ test, 0.97). No perfusion territory without WMAs imaged with use of the conventional technique was con- 
sidered abnormal at real-time imaging (Table 4). An example of new-onset hypokinesia during stress detected with use of both imaging techniques is shown in figure 2 .

Table 4: Real-time versus echo-planar imaging. Perfusion territories indicative of ischemia

\begin{tabular}{lll}
\hline \multirow{2}{*}{ RTI findings } & EPI findings & \\
\cline { 2 - 3 } \cline { 2 - 2 } Positive & Positive & Negative \\
\hline Negative & 22 & 0 \\
\hline
\end{tabular}

Note.-There were 66 perfusion territories. EPI = echo-planar imaging, RTI = real-time imaging.

In 16 patients, significant coronary arterial stenosis was detected with angiography. A sensitivity of $88 \%$ (14 of 16 patients) and a specificity of $83 \%$ (five of six patients) for diagnosing ischemia with echo-planar imaging and of 81\% (13 of 16 patients) and 83\% (five of six patients), respectively, with real-time MR imaging, when compared with coronary angiography, was found (Figure 3. Table 5).

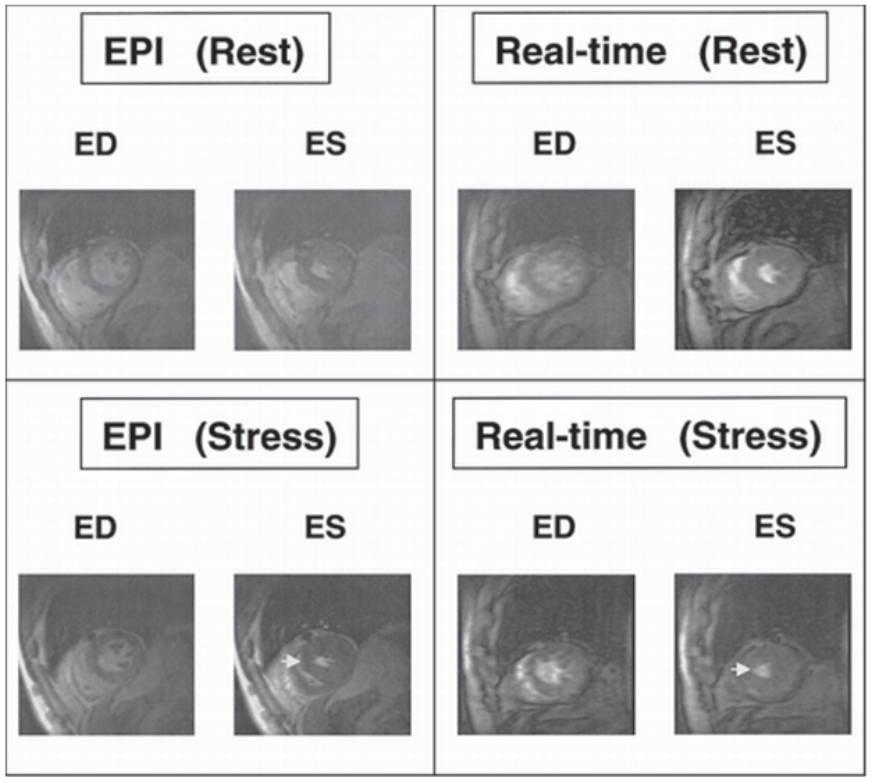

Figure 2: End-diastolic (ED) and end-systolic (ES) short-axis views obtained at the basal level by using echo-planar (EPI) and real-time MR imaging at rest and dobutamine hydrochloride stress ( $40 \mu \mathrm{g} / \mathrm{kg}$ with $0.5 \mathrm{mg}$ atropinsulfate): At rest, wall motion is normal, whereas during dobutamine hydrochloride stress, a new onset of hypokinesia (arrows) in the basal-septal segment is detected with both imaging techniques. 


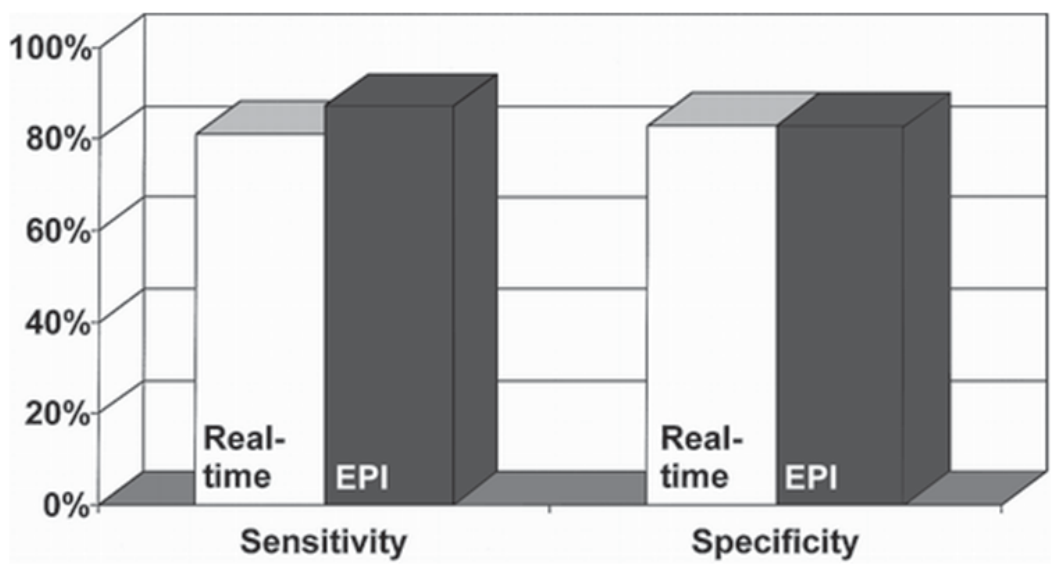

Figure 3: Graph shows the sensitivity and specificity of real-time and echo-planar imaging (EPI) for the diagnosis of ischemia, when compared with angiography. Detection of ischemia with real-time MR imaging is similar to that with standard echo-planar MR imaging.

Table 5: MR imaging versus angiography: Demonstration of coronary arterial stenosis with ischemia

\begin{tabular}{lcc}
\hline $\begin{array}{l}\text { MR imaging technique } \\
\text { and results }\end{array}$ & $\begin{array}{c}\text { Angiographic } \\
\text { stenosis } \geq 75 \%\end{array}$ & $\begin{array}{c}\text { Stenosis } \\
\text { not significant }\end{array}$ \\
\hline Echo-planar imaging & 14 & 1 \\
Ischemia detetced & 2 & 5 \\
Ischemia not detected & & 1 \\
Real-time imaging & 13 & 5 \\
Ischemia detected & 3 & \\
Ischemia not detected & & \\
\hline
\end{tabular}

Note.-Data are numbers of patients.

\section{Discussion}

Real-time MR image acquisition is similar to echo-planar imaging for detecting WMAs in patients known to have CAD. Real-time imaging is possible during high-dose dobutamine hydrochloride MR imaging. Image quality and contrast between blood and myocardium are sufficient to adequately assess wall motion and detect WMAs at rest or during pharmacologic stress.

In contrast with conventional MR imaging techniques, such as echo-planar or turbo gradient-echo imaging, ECG triggering is not necessary to preserve image quality, and high-quality images can be obtained even in patients with arrhythmia. Patients with atrial fibrillation, frequent premature heartbeats, or sinus arrhythmia ${ }^{27}$ may have moderately reduced MR image quality. With real-time imaging, image quality is independent 
of these pitfalls, as the complete data set is acquired in real-time (65 msec). In addition, breath holding is not necessary to preserve image quality.

A potential advantage is the ability to image the entire heart in 12-16 seconds, which may further improve diagnostic accuracy when compared with conventional MR imaging techniques that allow acquisition of only a limited number of views (eg, five) per stress level at current imaging speeds. In addition, real-time image acquisition may allow stress during ergometric exercise, which is regarded as the more physiologic stress test, with a better safety profile. ${ }^{28}$

A limitation of the study protocol was acquisition of real-time images at only two stress levels (20 $\mathrm{\mu g} / \mathrm{kg}$ and maximum dobutamine hydrochloride dose). This was due to patient safety considerations, since pharmacologic stress had to be kept to a minimum. These two stress levels were chosen to visualize wall motion at rest, at maximum inotropy without ischemia, and at maximum stress. Increasing contraction of segments during low-dose dobutamine hydrochloride stress or decreasing contraction during high-dose dobutamine hydrochloride stress may have been missed in some cases.

The patient population included in the current study was small and inhomogeneous; almost all patients had previously had a myocardial infarction, and most had undergone revascularization procedures, including coronary arterial bypass. Angiography was considered the reference standard for predicting ischemia, followed by impaired myocardial function in the current study, although coronary arterial patency or stenosis is not always accurately predictive of function. Patients with previous infarction who subsequently undergo angioplasty might have restoration of normal flow in the vessel but might still have impaired myocardial function in the perfusion territory as a result of scar tissue formation. In this specific patient population, results of real-time imaging were similar to those of echo-planar imaging, and a sensitivity of $81 \%$ and a specificity of $83 \%$ were found at comparison with angiography. Previous studies ${ }^{19}$ in which highdose dobutamine hydrochloride stress MR imaging was performed have been restricted to patients suspected of having CAD. Thus, the diagnostic accuracy of the current study cannot be compared with previous results and is expected to be lower. The value of real-time imaging as a screening test in patients suspected of having CAD remains to be determined in a future study.

Because of the high prevalence of patients who have multiple-vessel disease and have previously undergone coronary angioplasty or bypass surgery, defined assignment of segments to stenosed arteries was considered not useful for comparison of real-time and conventional MR imaging with coronary angiography.

Currently, some technical limitations need to be considered. The major limitation was the low spatial resolution $(2.2 \times 4.4$ for the real-time technique vs $1.3 \times 2.6 \mathrm{~mm})$ with the echo-planar imaging technique. Zero filling was used to better apply the information contained in the raw data ( $\mathrm{k}$ space) and to reduce partial volume effects and edge-detection artifacts. ${ }^{29,30}$ With this method, voxel size improved to $1.1 \times 2.2 \mathrm{~mm}$. This spatial resolution is probably not sufficient for detecting viable myocardium, since a 
dobutamine hydrochloride-induced increase in 2-mm or greater wall thickening is regarded as a diagnostic criterion for viability. ${ }^{31}$ However, for visual detection of WMAs, spatial resolution was sufficient, probably because of the eye's ability to interpret motion patterns rather than thickness alone.

At rest, the temporal resolution of $62 \mathrm{msec}$ used in the current study is just sufficient to acquire end-diastolic and end-systolic images at isovolumetric phases, since end-systole lasts approximately 50-80 msec, with a longer isovolumetric phase at enddiastole. During dobutamine hydrochloride stress, however, the isovolumetric phase is shortened and may be missed with the temporal resolution used in the current study. Acquiring several heartbeats for each section overcomes this problem, since different phases of the cardiac cycle are sampled in different heartbeats; thus, the chance of acquiring an image during maximum contraction is increased. These two limitations, low spatial and temporal resolution, may explain the small differences observed between echo-planar and real-time imaging.

The human eye is excellent at assessing abnormalities of complex motion patterns. However, limitations of visual interpretation of wall motion are well known. For example, changes in short-duration myocardial motion may be missed visually, even when an imaging modality with sufficient temporal resolution is used for data acquisition. ${ }^{32}$ Therefore, quantitative analysis of regional wall motion and WMAs would be helpful. Accurate determination of timing of regional motion events and regional myocardial wall thickening and thinning, as well as velocity and direction of myocardial motion in real-time, is a future goal. ${ }^{33}$ In the current setting, with suboptimal spatial and temporal resolution on the real-time MR images, quantitative analysis of WMAs was not considered useful.

A third technical limitation of the study was that, although images were acquired in real-time, reconstruction had to be performed offline because of hardware restrictions at the time of the study. Patients could not be monitored online for new onset of WMAs. Instead, monitoring was performed conventionally at imaging pauses, such as at the beginning of the next dobutamine hydrochloride stress level. However, at least in some patients, it was not possible to review the 16 segments without increasing the stress level duration to more than 3 minutes. Current patient monitoring is therefore suboptimal, since no sufficient image data is available during imaging. If this information were available, a stress test might be stopped earlier, since new WMAs are criteria for stopping the test. Thus, real-time imaging did not enable better monitoring of the patients in the current study, since only offline reconstruction was available and the value of real-time techniques was not known during the study. In the future, however, realtime images could be acquired continuously because of the latest hardware solutions, which already allow real-time reconstruction and interactive imaging ${ }^{23}$ and could therefore increase patient safety.

Two strategies may be used with real-time imaging for stress MR imaging examinations. The first strategy, which can be applied at present, is continuous visualization of 
cardiac motion in real-time to monitor the patient and rapidly detect signs of ischemia. High-spatial-resolution breath-hold imaging would be performed at 3-minute intervals for diagnosis. The second strategy is real-time imaging for diagnosing ischemia with use of pharmacologic or ergometric stress.

In conclusion, real-time MR imaging allows accurate detection of WMAs; therefore, it can be used for online analysis of wall motion with pharmacologic stress to reduce imaging time and improve patient safety and may allow use of physical stress. Because no data averaging or ECG triggering of several heartbeats is needed to use the real-time technique, it may be possible to perform imaging in patients who have atrial fibrillation or frequent premature heartbeats without loss of image quality. Real-time imaging may be an important addition to breath-hold MR imaging when hardware and software improvements allowing real-time image reconstruction become widely available.

\section{References}

1. Kaplan MA, Harris CN, Aronow WS, Parker DP, Ellestad MH. Inability of the submaximal treadmill stress test to predict the location of coronary disease. Circulation 1973; 47: 250-256.

2. Erbel R, Schweizer P, Meyer J, Krebs W, Yalkinoglu O, Effert S. Sensitivity of cross sectional echocardiography in detection of impaired global and regional left ventricular function: prospective study. Int J Cardiol 1985; 7: 375-389.

3. Crouse LI, Cheirif J, Hanly DE, et al. Opacification and border delineation improvement in patients with suboptimal endocardial border definition in routine echocardiography: results of the phase III albunex multicenter trial. J Am Coll Cardiol 1993; 22: 1494-1500.

4. Kornbluth $\mathrm{M}$, Liang $\mathrm{DH}$, Palome A, Schnittger I. Native tissue harmonic imaging improves endocardial border definition and visualization of cardiac structures. J Am Soc Echocardiogr 1998; 11: 693-701.

5. Kasprzak JD, Paelinck B, TenCate FJ, et al. Comparison of native and contrast-enhanced harmonic echocardiography for visualization of left ventricular endocardial border. Am J Cardiol 1999; 83: 211-217.

6 Longmore DB, Klipstein RH, Underwood SR, et al. Dimensional accuracy of magnetic resonance in studies of the heart. Lancet 1985; 1: 1360-1362.

7 Stratemeier EJ, Thompson R, Brady TJ, et al. Ejection fraction determination by MR imaging: comparison with left ventricular angiography. Radiology 1986; 158: 775-777.

8. Sechtem U, Pflugfelder PW, Gould RG, Cassidy MM, Higgins CB. Measurement of right and left ventricular volumes in healthy individuals with cine MR imaging. Radiology 1987; 167: 425-430.

9. Semelka RC, Tomei E, Wagner S, et al. Normal left ventricular dimensions and function: interstudy reproducibility of measurements with cine MR imaging. Radiology 1990; 174: 763-768.

10. Frahm J, Haase A, Matthaei D. Rapid NMR imaging of dynamic processes using the FLASH technique. Magn Reson Med 1986; 3: 321-327.

11. McDonald KM, Parrish T, Wennberg P, et al. Rapid, accurate and simultaneous noninvasive assessment of right and left ventricular mass with nuclear magnetic resonance imaging using the snapshot gradient method. J Am Coll Cardiol 1992; 19: 1601-1607.

12. Sakuma H, Fujita N, Foo TK, et al. Evaluation of left ventricular volume and mass with breath-hold cine MR imaging. Radiology 1993; 188: 377-380.

13. Bogaert JG, Bosmans HT, Rademakers FE, et al. Left ventricular quantification with breath-hold MR imaging: comparison with echocardiography. MAGMA 1995; 3: 5-12.

14. Hunter GJ, Hamberg LM, Weisskoff RM, Halpern EF, Brady TJ. Measurement of stroke volume and cardiac output within a single breath hold with echo-planar MR imaging. J Magn Reson Imaging 1994; 4: 51-58. 
15. Unterweger M, Debatin JF, Leung DA, Wildermuth S, McKinnon GC, von Schulthess GK. Cardiac volumetry: comparison of echoplanar and conventional cine-magnetic resonance data-acquisition strategies. Invest Radiol 1994; 29: 994-1000.

16. Wiesmann F, Gatehouse PD, Panting JR, Taylor AM, Firmin DN, Pennell DJ. Comparison of fast-spiral, echo-planar and fast low-angle shot magnetic resonance imaging for cardiac volumetry at $0.5 \mathrm{~T}$. J Magn Reson Imaging 1998; 8: 1033-1039.

17 Nagel E, Lehmkuhl HB, Boksch W, et al. High dose dobutamine magnetic resonance imaging for the detection of myocardial ischemia. Circulation 1999; 99: 763-770.

18. Hundley WG, Hamilton CA, Thomas MS, et al. Utility of fast cine magnetic resonance imaging and display for the detection of myocardial ischemia in patients not well suited for second harmonic stress echocardiography. Circulation 1999; 100: 1697-1702.

19. Nagel E, Lehmkuhl HB, Klein C, et al. Influence of image quality on the diagnostic accuracy of dobutamine stress magnetic resonance imaging in comparison with dobutamine stress echocardiography for the noninvasive detection of myocardial ischemia. Z Kardiol 1999; 88: 622-630. [German].

20. Zoghbi WA, Barasch E. Dobutamine magnetic resonance imaging: a serious contender in pharmacological stress imaging? Circulation 1999; 99: 730-732.

21. McKinnon GC. Ultrafast interleaved gradient-echo-planar imaging on a standard scanner. Magn Reson Med 1993; 30: 609-616.

22. Nagel E, Schneider U, Schalla S, et al. Magnetic resonance real-time imaging for the evaluation of left ventricular function. J Cardiovasc Magn Reson 2000; 2: 7-14.

23. Yang $P$, Kerr A, Liu A, et al. New real-time interactive cardiac magnetic resonance imaging system complements echocardiography. J Am Coll Cardiol 1999; 32: 2049-2056.

24. Holland AE, Goldfarb JW, Edelman RR. Diaphragmatic and cardiac motion during suspended breathing: preliminary experience and implications for breath-hold MR imaging. Radiology 1998; 209: 483-489.

25. Schiller NB, Shah PM, Crawford M, et al. Recommendations for quantitation of the left ventricle by twodimensional echocardiography: American Society of Echocardiography Committee on Standards, Subcommittee on Quantitation of Two-Dimensional Echocardiograms. J Am Soc Echocardiogr 1989; 2: 358367.

26. Segar DS, Brown SE, Sawada SG, Ryan T, Feigenbaum H. Dobutamine stress echocardiography: correlation with coronary lesion severity as determined by quantitative angiography. J Am Coll Cardiol 1992; 19: 1197-1202.

27. Van Rugge FP, Holman ER, van der Wall EE, de Roos A, van der Laarse A, Bruschke AV. Quantitation of global and regional left ventricular function by cine magnetic resonance imaging during dobutamine stress in normal human subjects. Eur Heart J 1993; 14: 456-463.

28. Geleijnse ML, Fioretti PM, Roelandt JR. Methodology, feasibility, safety and diagnostic accuracy of dobutamine stress echocardiography. J Am Coll Cardiol 1997; 30: 595-606.

29. Parker DL, Yiping DP, Davis DL. The voxel sensitivity function in fourier transform imaging: applications to magnetic resonance angiography. Magn Reson Med 1995; 33: 156-162.

30. Parker DL, Parker DJ, Blatter DD, Yiping PD, Goodrich KC. The effect of image resolution on vessel signal in high-resolution magnetic resonance angiography. J Magn Reson Imaging 1996; 6: 632-641.

31. Baer FM, Theissen P, Schneider CA, et al. Dobutamine magnetic resonance imaging predicts contractile recovery of chronically dysfunctional myocardium after successful revascularization. J Am Coll Cardiol 1998; 31: 1040-1048.

32. Kvitting JPE, Wigstrom L, Strotmann JM, Sutherland GR. How accurate is visual assessment of synchronicity in myocardial motion? An in vitro study with computer simulated regional delay in myocardial motion: clinical implications for rest and stress echocardiography studies. J Am Soc Echocardiogr 1999; 12: 698705.

33. Hatle L, Sutherland GR. Regional myocardial function: a new approach. Eur Heart J 2000; 21: 1337-1357. 


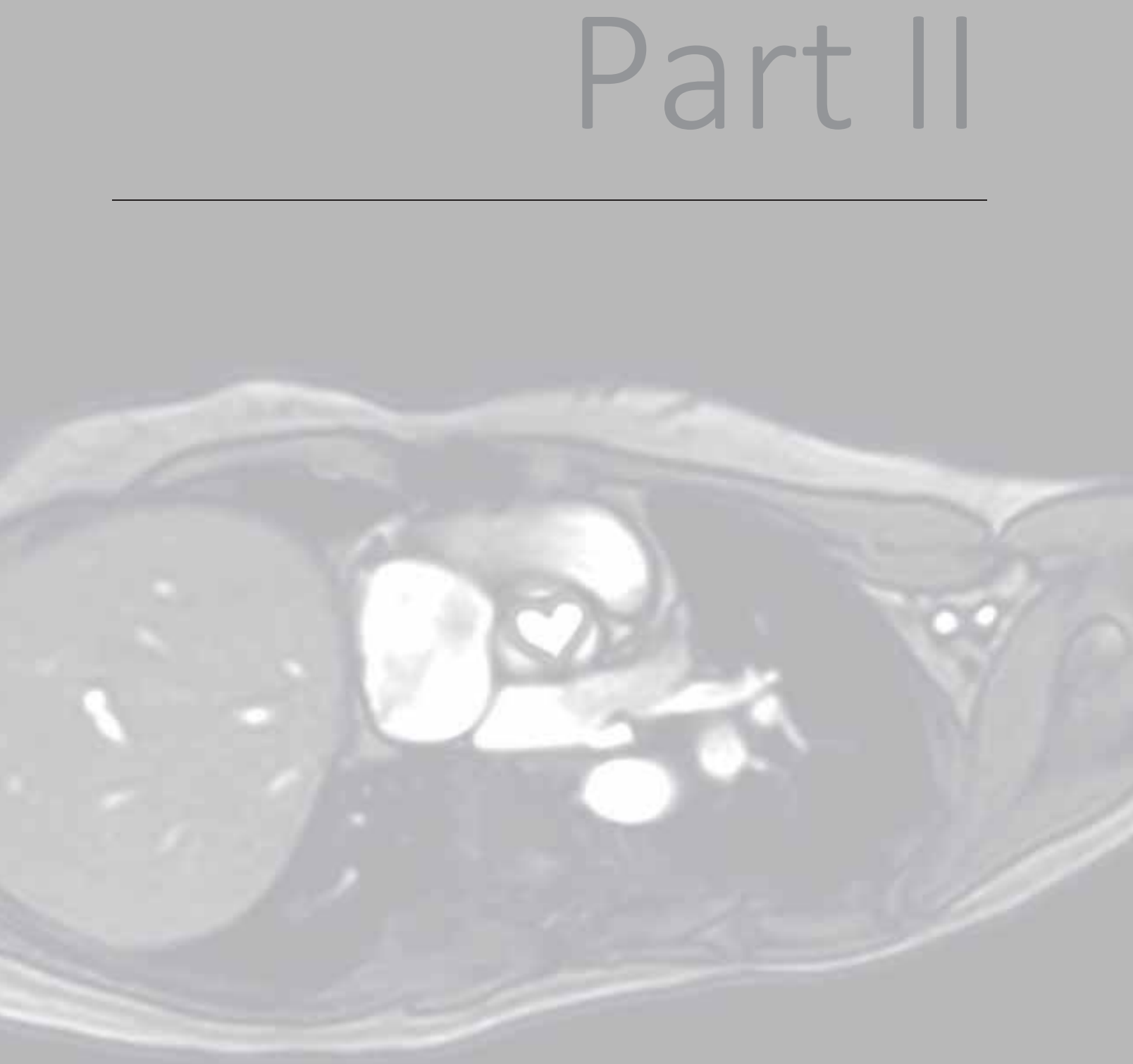



\section{Accentuation of high susceptibility of}

hypertrophied myocardium to ischemia:

Complementary assessment of Gadophrin-enhancement and left ventricular function with MRI 


\section{Abstract}

Background: The aim of the study was to compare infarction size and left ventricular (LV) function in normal and hypertrophied hearts after brief ischemia using Gadophrinenhancement and functional assessment by MRI.

Methods: Rats ( $n=20$ ) were assigned to aortic banding to induce LV hypertrophy or control. Eight weeks later, rats were subjected to $25 \mathrm{~min}$ of regional myocardial ischemia followed by $3 \mathrm{hr}$ of reperfusion. The necrosis-specific agent Gadophrin-3 was injected to delineate infarcted myocardium on MRI. Effects of aortic banding and ischemia on LV mass and function were determined. At postmortem, areas at risk and infarction were measured.

Results: A close correlation was found between LV mass measured with MRI and at postmortem $(r=0.98)$. LV mass measured with MRI was significantly greater $(0.81 \pm$ $0.02 \mathrm{~g})$ in animals with aortic banding compared to control $(0.62 \pm 0.02 \mathrm{~g}$; $\mathrm{P}<0.001)$. Infarction size was larger in hypertrophied hearts $(19.0 \pm 1.4 \% / 18.3 \pm 1.5 \%)$ than in control $(9.8 \pm 1.7 \%$ / $9.2 \pm 2.0 \%)$ on Gadophrin-enhanced MRI and at postmortem, respectively. Similarly, greater impairment in ejection fraction was observed in hypertrophied hearts with MRI ( $39 \pm 4 \%$ vs. $49 \pm 2 \%$; $P=0.02)$.

Conclusions: Gadophrin-3 provides accurate estimation of infarct size in hypertrophied hearts. Hypertrophied hearts are more sensitive to ischemia than nonhypertrophied hearts. The complementary assessment of Gadophrin-enhancement and LV function with MRI provides unique information about myocardium sensitivity to ischemia. 


\section{Introduction}

Left ventricular (LV) hypertrophy is one of the major risk factors for myocardial infarction, congestive heart failure, sudden death, and other cardiovascular mortality and morbidity. ${ }^{1,2}$ Therefore, early detection of LV hypertrophy is important in patients with hypertension and vascular disease. MRI has the capability to noninvasively and sequentially measure LV mass and function. ${ }^{3-5}$

Physiologic studies have shown that hypertrophied hearts are prone to greater susceptibility to ischemic injury than nonhypertrophied hearts. ${ }^{6,7}$ Imbalance between myocardial mass and oxygen supply is the possible mechanism leading to larger and more frequent infarctions in patients with hypertrophied hearts. Microscopic studies have shown that the increase in heart weight in hypertrophied hearts can be attributed to an increase in myocyte size, proliferation of nonmyocytes, and water accumulation. ${ }^{8}$ Hypertrophied hearts are characterized by an increase in coronary vascular permeability and collagen accumulation paralleled with a decrease in capillary density. ${ }^{9}$ These micromorphologic changes in myocardium lead to an increase in oxygen diffusion distance and to vascular resistance and stiffness. ${ }^{10}$ It has also been suggested that alterations in myocardial energy metabolism, such as glucose metabolism, might increase the susceptibility of hypertrophied hearts to ischemia. ${ }^{11}$

Determination of the infarction size in the acute phase is important for therapeutic decisions and patient prognosis. In acute myocardial infarction, the use of extracellular contrast agents for precise determination of the spatial extent of necrotic myocardium is controversial. Previous MR studies in nonhypertrophied and hypertrophied rat hearts have indicated that infarction size is overestimated after administration of extracellular contrast media. ${ }^{12-15}$ On the other hand, recent MR studies have demonstrated the high accuracy of necrosis-specific contrast media, Gadophrin-2 and 3, in delineating acute myocardial infarction of nonhypertrophied hearts, ${ }^{13,14,16,17}$ but not in hypertrophied hearts. Furthermore, Gadophrin-3 has not been used to correlate the infarction size with LV function in hypertrophied hearts using MRI.

Thus, the purposes of this MR study were to:

1. Determine and compare the effect of brief ischemic episodes on the infarction size in hypertrophied and nonhypertrophied hearts using Gadophrin-3. The true infarction size measured at postmortem with TTC was compared to the Gadophrin-3enhanced regions on MRI.

2. Determine and compare the effect of ischemia on regional (wall thickness and thickening) and global LV function in hypertrophied and nonhypertrophied hearts. 


\section{Methods}

\section{Animal preparation}

Sprague-Dawley rats ( $n=20$, female) were studied in accordance with the National Institutes of Health Guide for the Care and Use of Laboratory Animals and with the approval of the Committee of Animal Research of the university. Rats were anesthetized with a mixture of ketamine $(50 \mathrm{mg} / \mathrm{kg}$, Ketaset, Fort Dodge, Fort Dodge, IA) and xylazine (1.4 mg/kg, Xyla-ject, Phoenix, St. Joseph, MO) injected intraperitoneally. In 10 young rats (110-120 g), the aorta was isolated from the surrounding adipose tissue via right laparotomy. A sterile steel wire of $0.4 \mathrm{~mm}$ diameter was placed close to the surface of the aorta and a thread was passed around the aorta and the wire. The thread was tied firmly around the aorta and the wire. The wire was pulled back to restore the flow in the aorta. Successful aortic stenosis was assured by visual inspection of the pale color of the kidneys. Removal of the wire resulted in returning of the red color to the kidneys. The site of the incision was closed by multilayer suturing. The rats were kept for 8 weeks under controlled food, light, and temperature conditions to develop LV hypertrophy. Another 10 rats with matched body weight (100-120 g) and environmental conditions were used as a control (no aortic stenosis).

Eight weeks later, MRI was performed. Prior to MRI the rats were anesthetized with an intraperitoneal injection of sodium pentobarbital $(50 \mathrm{mg} / \mathrm{kg}$, Nembutal Sodium Solution, Abbot Laboratories, North Chicago, IL) followed by intravenous ( $6 \mathrm{mg} / \mathrm{kg} / \mathrm{hr}$ ) doses to maintain the depth of anesthesia during coronary artery occlusion, reperfusion, MRI, and pressure measurements. After tracheostomy, a left thoracotomy was performed under mechanical ventilation with room air (Harvard Apparatus, South Natick, MA). The hearts were then subjected to regional ischemia by occluding the anterior branch of the left coronary artery for 25 min with a 5.0 prolene snare loop followed by a 3-hr reperfusion.

Necrosis-specific contrast agent for T1-weighted MRI

The necrosis-specific agent Gadophrin-3 (\{mu-[\{16,16'-[(7,12-Diethyl-3,8,13,17-tetramethyl-21H,23H-porphine-2,18-diyl-N21,N22,N23,N24)copper]bis[3,6,9-tris(carboxymethyl)11,14-dioxo-3,6,9,12,13-pentaazahexadecanoato]\}(8-)]\}digadolinate(2-), disodium, Schering AG, Berlin, Germany) is a porphyrin-based T1-enhancing gadolinium agent. The physicochemical and pharmacological properties of Gadophrin-3 are comparable to Gadophrin-2, with T1 and T2 relaxivities of 8.9 and 12 sec-1 mM-1. ${ }^{13,14,16,18}$ Due to the addition of a copper atom to the center of the porphyrin molecule, stability was improved. Gadophrin-3 was injected intravenously $(0.05 \mathrm{mmol} / \mathrm{kg}) 5 \mathrm{~min}$ after reperfusion ( $3 \mathrm{hr}$ prior to imaging). During the reperfusion period the contrast agent accumulates in infarcted myocardium and clears from the plasma. Since the plasma half-life of 
this agent is $90 \mathrm{~min}$, imaging at an earlier time after administration provides similar information as extracellular T1-enhancing contrast agents about infarction size. Unlike extracellular agents, Gadophrin-based agents bind to necrotic tissue or cellular debris. ${ }^{19}$

$M R I$

MRI was performed $3 \mathrm{hr}$ after Gadophrin-3 injection. Sodium pentobarbitalanesthetized rats were placed supine in a custom-made birdcage RF coil of $5.6 \mathrm{~cm}$ inner diameter and $7.6 \mathrm{~cm}$ length. Multislice T1-weighted spin echo short axis images were acquired in end-systole and end-diastole to define the infarcted regions in the entire heart and to obtain LV volumes, mass, and wall thickness. A 2.0 T CSI-II-system (Bruker Instruments, Fremont, CA) was used for image acquisition with the following parameters: $6-8$ contiguous short axis slices to cover the entire heart with slice thickness $=2$ $\mathrm{mm}$, field of view $=5 \times 5 \mathrm{~cm}, \mathrm{TR}=300 \mathrm{~ms}, \mathrm{TE}=12 \mathrm{~ms}$, image matrix $=256 \times 128$, number of excitations $=4$, and acquisition time $=3-3.5 \mathrm{~min}$, depending on the heart rate. ECG-gating was enabled by placing two copper needles in the forelimb and chest wall connected to an electrocardiographic monitor (Accusync 6L, AMR, Milford, CT). Enddiastolic images were acquired at the origin of $\mathrm{R}$ wave and end-systolic images at $45 \%$ of the RR interval. ${ }^{13}$

\section{Hemodynamic measurements}

Hemodynamic measurements were obtained in all animals in less than 10 min after MRI. The right carotid artery was catheterized and the catheter advanced into the aorta and LV in order to obtain arterial and cardiac pressures. Pressures were measured by means of a saline-filled catheter and pressure transducer. Monitoring the pressurewave signal ensured correct placement. A special pressure transducer for rodents was used to monitor the changes in pressure (Model P-10EZ Spectramed Stathan; Gould, Cleveland, $\mathrm{OH}$ ). The pressure transducer was calibrated and standardized prior to each experiment. Positive and negative maximum rate of LV pressure development (differential quotient of pressure change against time, $\mathrm{dP} / \mathrm{dt}$ ) was electronically derived from the pressure wave signal of LV.

\section{Postmortem validation}

After the hemodynamic measurements the coronary artery was reoccluded by the same snare occluder. Phthalocyanine blue dye $(0.7 \mathrm{ml})$ was intravenously injected to delineate the area at risk. The area at risk supplied by the occluded coronary artery remained unstained while perfused territories were stained blue. The heart was excised, the RV and atria removed, and the LV weighed to determine LV mass. The LV was then sliced in the short-axis view into 6-7 slices; each of $2 \mathrm{~mm}$ thickness corresponding to the MR 
images. All slices were then incubated for $10 \mathrm{~min}$ in $2 \%$ triphenyltetrazolium chloride (TTC) solution (Sigma Chemical Co., St. Louis, MO) to discriminate infarcted myocardium (pale region) from noninfarcted myocardium (brick red region). Digital photography pictures were taken from both sides of the slices. Areas at risk for infarction and infarction size were determined in all animals.

Image Analysis

MR images and postmortem photos were analyzed with the NIH Image software tool (National Institutes of Health, Bethesda, MD). On MR images, endocardial and epicardial borders and enhanced areas of the LV were manually traced to determine LV volumes, mass, and infarction size (planimetry). The volume per slice was obtained by multiplying with the slice thickness. End-diastolic and end-systolic volumes were then calculated by adding together the volumes of all short-axis slices (Simpson method). Ejection fraction was calculated as: (end-diastolic volume - end-systolic volume)/end-diastolic volume. LV mass was calculated at end-diastole by subtracting the end-diastolic endocardial volume from the end-diastolic epicardial volume and then multiplying by the density of the myocardium (1.05 g/cm3). The borders between infarcted and normal myocardium were clearly visible and allowed measurement of infarction size on end-diastolic images by manual tracing. LV wall thickness $(\mathrm{mm})$ was measured on end-systolic and enddiastolic images in a remote region (septal wall) and in the center of infarction on two slices per animal. Percent systolic wall thickening was calculated as: [(systolic wall thickness - diastolic wall thickness)/diastolic wall thickness] $\times 100$. Signal intensity (SI) was determined in all short-axis slices with infarcted areas in both animal groups. The borders of the contrast-enhanced area of each slice was manually traced and the mean signal intensity determined. Results were divided by SI determined in a remote area (posterior wall) of the same slice. Stroke volume (SV) in $\mathrm{ml}$ was calculated from MR data as end-diastolic volume minus end-systolic volume. Cardiac output in $\mathrm{ml} / \mathrm{min}$ was calculated as SV multiplied by heart rate. Systemic vascular resistance in $\mathrm{mmHg} / \mathrm{l} / \mathrm{min}$ was calculated from MR data and pressure measurements as mean aortic pressure divided by cardiac output.

\section{Statistical analysis}

All parameters are expressed as mean values \pm standard error of the mean. The unpaired two-sample Student's t-test was used to compare the differences in infarction size, LV mass and function, and body weight between control and hypertrophy groups when measured with the same method. The null hypothesis was rejected when the $P$ value was less than 0.05. Bland-Altman analysis (mean \pm 2 SDs) was used to determine the agreement between MR and postmortem measurements in both control and animals with myocardial hypertrophy. The linear regression line obtained from the data of 
both animal groups was constructed to determine the correlation coefficient values between MR and postmortem measurements. Linear regression was also obtained to determine the correlation values between MRI measurements of EF and infarction size.

\section{Results}

\section{LV mass}

The LV mass determined with MRI was significantly greater in rats subjected to aortic stenosis $(0.805 \pm 0.015 \mathrm{mg})$ than in control animals $(0.618 \pm 0.053 \mathrm{~g} ; \mathrm{P}<0.0001)$ (Table 1). LV mass measured at postmortem was also significantly greater in hypertrophied hearts $(0.818 \pm 0.014 \mathrm{~g}$ in hypertrophied and $0.614 \pm 0.016 \mathrm{~g}$ in nonhypertrophied hearts at postmortem, $\mathrm{P}<0.0001)$. Regression analysis indicated excellent correlation between MR mass and postmortem mass ( $r=0.98$; standard error of the estimate (SEE) $=0.02 ; y=0.93 x+0.05)$. Bland-Altman analysis revealed an excellent agreement between the mass determined with MRI for hypertrophy $(-0.013 \pm 0.040 \mathrm{~g}(2 \mathrm{SD}))$ and for control group $(0.004 \pm 0.025 \mathrm{~g}(2 \mathrm{SD}))$ in comparison with postmortem (Fig. 1). The number of short axis slices required to cover the entire heart was 1-2 slices greater in hypertrophied hearts (Fig. 2). The body weight of the two groups was similar at the time of infarction (244.7 $\pm 4.1 \mathrm{~g}$ in animals with hypertrophied hearts and $243.4 \pm 6.7 \mathrm{~g}$ in control animals, $\mathrm{P}=0.87$ ).

Infarction size (Gadophrin MRI vs. histochemical staining)

MR images acquired $3 \mathrm{hr}$ after the administration of Gadophrin-3 revealed that the infarcted regions appeared bright in hypertrophied and nonhypertrophied hearts. The SI ratios of infarcted and remote myocardium were $1.86 \pm 0.07$ in hypertrophied hearts and $1.82 \pm 0.16$ in nonhypertrophied hearts $(P=0.83)$. Therefore, the difference in size of infarction cannot be attributed to the magnitude of contrast enhancement of the injured regions. However, the size of the infarcted region was significantly larger in hypertrophied hearts $(19.0 \pm 1.4 \%$ of LV) than in nonhypertrophied hearts $(9.8 \pm 1.7 \%$ of LV, P $<0.001$ ) on MR images (Fig. 3). 
Table 1: MRI and postmortem data

\begin{tabular}{lccc}
\hline & Control & Hypertrophy & $P$ \\
\hline Body weight [g] & $243.4 \pm 6.7$ & $244.7 \pm 4.1$ & 0.87 \\
MR LV mass [g] & $0.618 \pm 0.053$ & $0.805 \pm 0.015$ & $<0.0001$ \\
Postmortem LV mass [g] & $0.614 \pm 0.016$ & $0.818 \pm 0.016$ & $<0.0001$ \\
MR infarction [\% LV] & $9.8 \pm 1.7$ & $19.0 \pm 1.4$ & $<0.001$ \\
Postmortem infarction [\%LV] & $9.2 \pm 2.0$ & $18.3 \pm 1.5$ & $<0.01$ \\
Postmortem area at risk [\%LV] & $37.0 \pm 1.5$ & $38.5 \pm 1.5$ & 0.50 \\
Wall thickness remote [mm] & $2.39 \pm 0.07$ & $2.85 \pm 0.11$ & $<0.01$ \\
Wall thickness infarct [mm] & $2.49 \pm 0.05$ & $2.92 \pm 0.11$ & $<0.01$ \\
\%WT remote [\%] & $34.7 \pm 2.0$ & $27.5 \pm 2.4$ & 0.043 \\
\%WT infarct [\%] & $3.7 \pm 0.9$ & $4.1 \pm 2.3$ & 0.88 \\
\hline
\end{tabular}

Mean \pm standard error of the mean; MR LV mass, left ventricular mass determined on MR images; \%LV, area in percent of left ventricle; wall thickness, diastolic wall thickness in remote and infarcted region; \%WT, percent wall thickening.
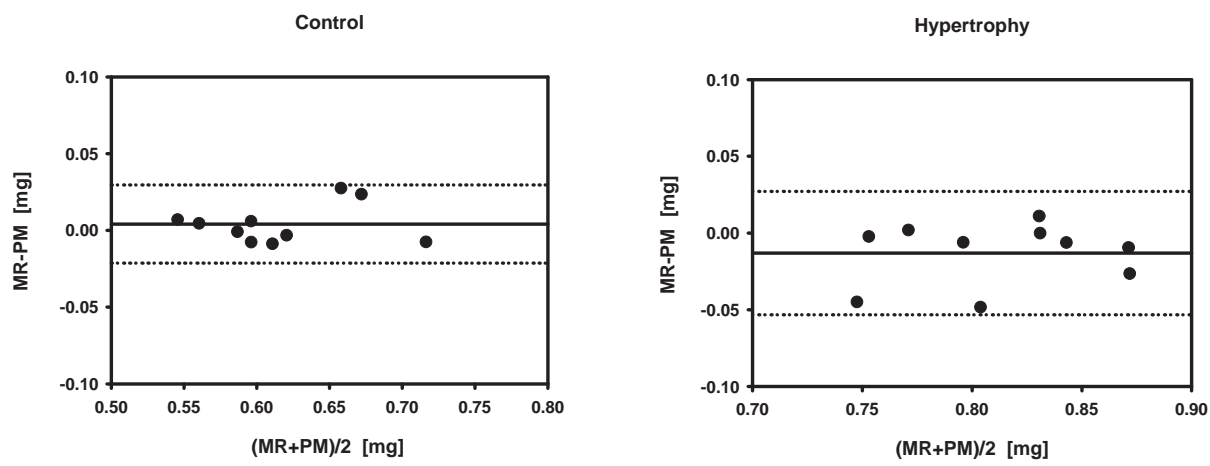

Figure 1: Bland-Altman scatterplots of left ventricular mass determined with MRI and at postmortem (PM) for control nonhypertrophied (left) and hypertrophied (right) hearts.
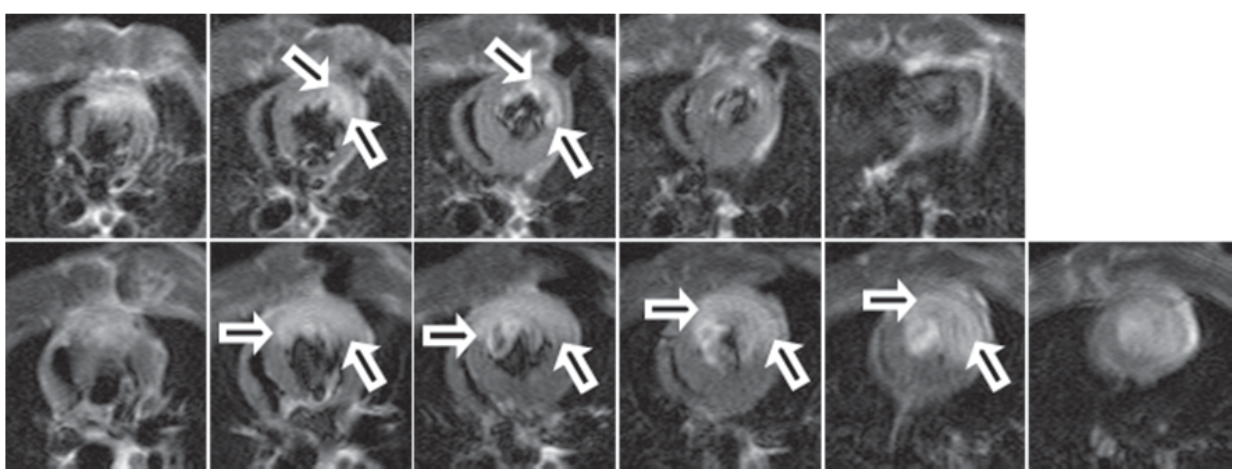

Figure 2: T1-weighted spin echo MR images: short axis slices of a control nonhypertrophied heart subjected to $25 \mathrm{~min}$ ischemia and $3 \mathrm{hr}$ reperfusion (top image row) and a hypertrophied heart subjected to the same duration of ischemia/reperfusion (bottom image row) after administration of Gadophrin-3. The size of the hyperenhanced zone is larger in hypertrophied heart. 


\section{Hypertrophy}

Control
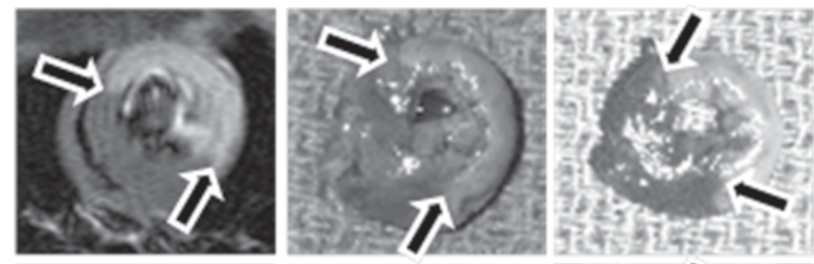

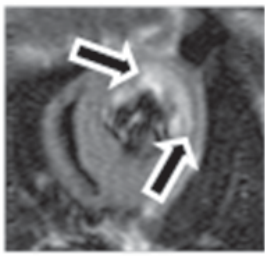

MRI

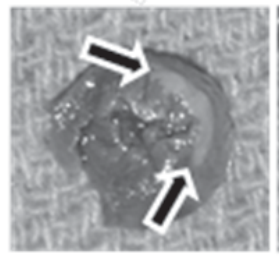

TT

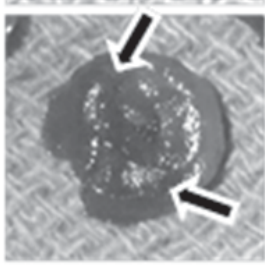

Blue Dye

Figure 3: Comparison between the extent of Gadophrin-3 enhanced regions (left), true infarction size after triphenyl-tetrazolium-chloride (TTC) staining (middle) and area at risk after phthalocyanine blue-dye staining at postmortem (right) in control and hypertrophied rat hearts. Both contrast-enhanced region and true infarction size were larger in hypertrophied hearts, while the area at risk was similar in comparison with control hearts.

At postmortem (TTC staining), the location of the infarction was identical to that seen on MRI. The infarction size was $18.3 \pm 1.5 \%$ LV in hypertrophied and $9.2 \pm 2.0 \%$ LV in nonhypertrophied hearts $(P=0.002)$. Regression analysis showed excellent correlation between Gadophrin-3-enhanced regions and histochemical staining at postmortem ( $r=$ 0.98; $\mathrm{SEE}=1.132 ; \mathrm{y}=1.02 \mathrm{x}+0.08)$. Bland-Altman test revealed excellent agreement between the size of infarction seen on Gadophrin-3-enhanced MR images and that on histochemical staining (hypertrophy: $0.72 \pm 2.94 \%$; normal: $0.10 \pm 0.98 \%$ ) (Fig. 4). Both animal groups had identical areas at risk $(38.5 \pm 1.5 \%$ LV in hypertrophied hearts and $37.0 \pm 1.5 \%$ in nonhypertrophied hearts, $P=0.50$ ).
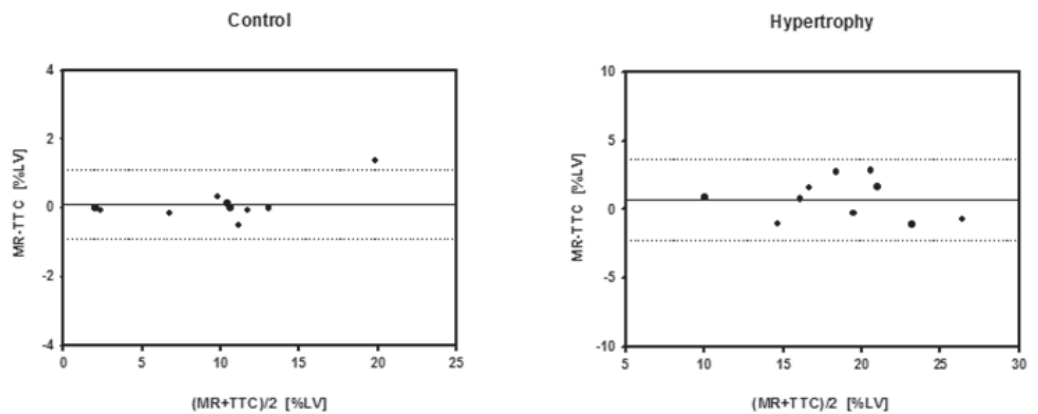

Figure 4: Bland-Altman scatterplots of infarction size measured with MRI and postmortem histochemical staining (PM) for control nonhypertrophied (left) and hypertrophied (right) hearts. [\% LV] = percentage of left ventricle defined by enhancement or staining. 


\section{Cardiac function}

The impairment in LV function was more pronounced in hypertrophied hearts than nonhypertrophied hearts subjected to the same duration of ischemia/reperfusion. The ejection fraction was significantly lower in hypertrophied (38.6 $\pm 3.7 \%)$ than nonhypertrophied hearts $(48.7 \pm 1.7 \%$; $P=0.02)$. LV dilation after coronary occlusion/reperfusion was greater in hypertrophied than nonhypertrophied hearts, as reflected by the enddiastolic volume (EDV). The EDV was $0.21 \pm 0.02 \mathrm{ml}$ in hypertrophied and $0.16 \pm 0.01 \mathrm{ml}$ in nonhypertrophied hearts $(P=0.03)$. The results of linear regression between ejection fraction and infarction size were: $r=0.74 ; \mathrm{SEE}=4.66 ; y=-0.49 x+35.69$ (Fig. 5).

Diastolic wall thickness in remote and infarcted myocardium was significantly different between the two groups. In this acute infarct state no differences were observed between the groups for \%wall thickening in infarcted myocardium (Table 1). In remote myocardium, percentage wall thickening was higher in the control group (34.7 \pm 2.0 vs. $27.5 \pm 2.4, P=0.04)$. Hemodynamic parameters derived from MR and LV catheter measurements are given in Table 2 . The mean heart rate did not differ between MRI and pressure measurements.

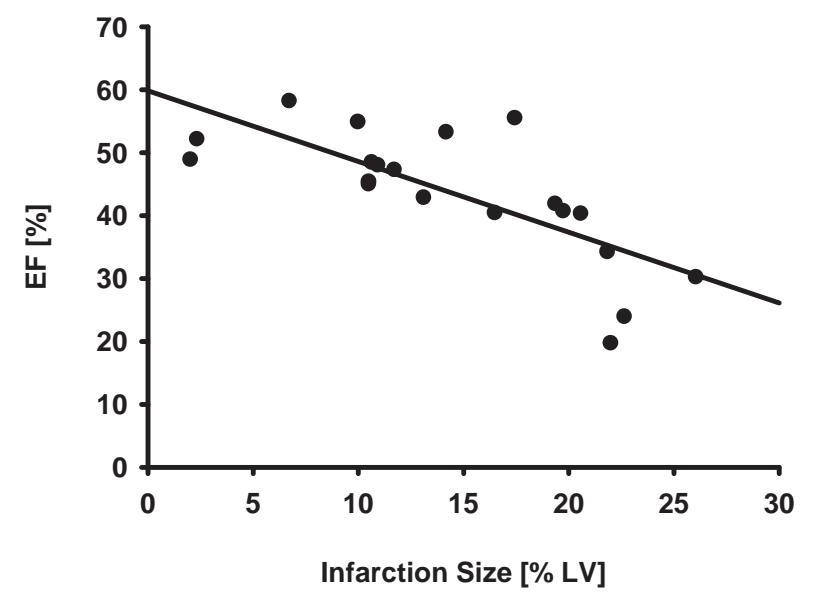

Figure 5: Linear regression: left ventricular ejection fraction (EF) vs. infarction size determined with MRI $(n=22$, control nonhypertrophied and hypertrophied rat hearts). 
Table 2: Hemodynamic parameter from MRI and catheter measurements

\begin{tabular}{lccc}
\hline & Control & Hypertrophy & $P$ \\
\hline HR [/min] & $386 \pm 9$ & $354 \pm 13$ & 0.06 \\
Mean BP $[\mathrm{mmHg}]$ & $80 \pm 5$ & $109 \pm 3$ & 0.01 \\
LVEDP $[\mathrm{mmHg}]$ & $5 \pm 1$ & $12 \pm 2$ & 0.03 \\
$+\mathrm{dP} / \mathrm{dt}$ & $9200 \pm 962$ & $4875 \pm 1299$ & 0.02 \\
$-\mathrm{dP} / \mathrm{dt}$ & $-8500 \pm 1369$ & $-4125 \pm 640$ & 0.02 \\
EDV [ml] & $0.16 \pm 0.01$ & $0.21 \pm 0.02$ & 0.03 \\
EF [\%] & $49 \pm 2$ & $39 \pm 4$ & 0.02 \\
SV [ml] & $0.08 \pm 0.01$ & $0.08 \pm 0.01$ & 0.97 \\
CO [ml/min] & $30.7 \pm 1.7$ & $28.2 \pm 2.9$ & 0.49 \\
SVR $[\mathrm{mmHg} / \mathrm{l} / \mathrm{min}]$ & $2653 \pm 110$ & $3876 \pm 52$ & $<0.0001$ \\
\hline
\end{tabular}

Values are mean \pm standard error of the mean for control and animals with hypertrophied myocardium (hypertrophy). HR, heart rate; Mean BP, mean aortic pressure. LVEDP, left ventricular end-diastolic pressure; $+/-\mathrm{dP} / \mathrm{dt}$, maximal rates of left ventricular pressure rise and decline; EDV, end-diastolic left ventricular volume; EF, ejection fraction; SV, stroke volume; CO, cardiac output; SVR, systemic vascular resistance.

\section{Discussion}

The major findings of this study were that 1) the infarction size was almost doubled in hypertrophied hearts compared to nonhypertrophied hearts after a brief episode of ischemia, suggesting the high sensitivity of hypertrophied hearts to ischemia; and 2) the complementary assessment of Gadophrin-enhancement and LV function with MRI provides unique information about myocardium sensitivity to ischemia.

The model examined in the current study resembles a hypertrophied heart with reperfused acute infarction. Pressure overload from aortic banding induces cardiac hypertrophy as an adaptive physiologic response at early stages, but is in the later course associated with dysfunction or heart failure. ${ }^{20,21}$ Activation of the sympathetic nervous system and renin-angiotensin system are considered important factors in this process, but the exact underlying mechanisms are unknown. ${ }^{22}$ Although abdominal banding activates the renin-angiotensin system, ${ }^{23}$ pressure overload alone was able to induce cardiac hypertrophy in an angiotensin receptor knockout mouse model, suggesting that high blood pressure with hemodynamic stress is one of the etiologic factors. ${ }^{24}$ Other studies have shown that lowering blood pressure with amlodipine therapy or inhibition of nitric oxide synthesis did not attenuate the increase in heart weight associated with aortic banding. ${ }^{25,26} \mathrm{MRI}$ and echocardiography are used as noninvasive methods to determine LV mass. MRI is considered more accurate due to a 3D technique compared with the m-mode and $2 \mathrm{D}$ area length formulas of echocardiography. ${ }^{27}$ In addition, MRI has also proven its value in patients with LV hypertrophy. ${ }^{28}$ The results of our study confirm the close correlation between MR and postmortem LV mass. 
Myocardial infarction can be recognized noninvasively as a perfusion deficit with single photon emission computed tomography or $\mathrm{MRI}^{29}$ as a wall motion abnormality with echocardiography or $\mathrm{MRI}^{30}$ and as late enhancement of chronic infarction with MRI using extracellular MR contrast agents. ${ }^{31}$ However, it currently remains controversial whether extracellular contrast agents have the potential to precisely define the size of acute infarction and whether the time delay between application of extracellular contrast agents and MRI is critical. ${ }^{32}$ Saeed et al. ${ }^{13}$ and Jeong et al. ${ }^{33}$ found overestimation of infarction size using extracellular contrast agent. Oshinski et al. ${ }^{15}$ presented data suggesting that time delay is also critical for sizing of subacute infarction. Barkhausen et al. ${ }^{17}$ found no difference in infarction size after Gd-DTPA or Gadophrin-3-enhanced MRI. However, in that study Gadophrin-enhanced MRI was employed in six rabbits subjected to complete coronary artery occlusion for $48 \mathrm{hr}$ without reperfusion. Because rabbit hearts show almost no collateral circulation, ${ }^{34}$ the entire area at risk was subjected to necrosis and a substantial peri-infarction zone will not be present after this long occlusion period. The limiting factor in using extracellular MR contrast agents for sizing of acute infarction is the time delay between contrast agent injection and MRI: the washout of these contrast agents varies from case to case. Solving this problem will make extracellular agents suitable for sizing of acute infarction. A major advantage of extracellular contrast agents is that they are, unlike necrosis-specific agents, approved for clinical use. However, precise determination of necrotic areas was critical in the current study to allow comparison of infarction size between hypertrophied and nonhypertrophied hearts. Therefore, MRI with necrosis-specific contrast agents was employed in this study. This class of contrast agents provided a close correlation between myocardial necrosis after acute infarction measured on MR images and at postmortem. ${ }^{13,16,18}$ The time delay between administration of necrosis-specific contrast agents and MRI is not important for sizing of acutely infarcted myocardium because these agents bind to necrotic tissue for a prolonged period of time. ${ }^{18}$

The greater susceptibility to ischemia in pressure overload hypertrophied compared to normal hearts has been described. ${ }^{6,35}$ In nonhypertrophied hearts, ischemia induces multiple changes in myocardial metabolism, including a marked increase in glucose uptake because of translocation of insulin-sensitive glucose transporters from intracellular membrane storage vesicles to the sarcolemma. ${ }^{36}$ This response promotes glycolysis during ischemia and early reperfusion, thereby facilitating production of high-energy phosphates through anaerobic and aerobic glycolysis. Hypertrophied hearts exhibit an accelerated loss of high-energy phosphates during ischemia, earlier onset of ischemic contracture, and decreased recovery of contractile function after reperfusion compared with nonhypertrophied hearts. ${ }^{37,38}$ Using MRI of cardiac function, we found that the impairment in LV function was more pronounced in hypertrophied than normal hearts. A potential limitation of the study is that MRI prior to myocardial ischemia was not performed and, thus, the effect of aortic banding on LV function prior to infarction was not assessed. However, animals without cardiac hypertrophy already showed impaired 
EF after infarction. Animals with hypertrophied hearts showed larger infarction size and, thus, are expected to have more severe impairment of EF due to ischemia alone.

The necrosis-specific agent Gadophrin-3 was used for the first time in sizing myocardial infarction in hypertrophied myocardium. Lauerma et al. ${ }^{12}$ performed MRI in conjunction with the extracellular MR contrast medium Gadoteridol to measure the infarction size in hypertrophied rat hearts. They found that Gadoteridol overestimated the true infarction (TTC infarction) by $30 \%$. Necrosis-specific MR contrast agents represented by Gadophrin-2 and 3 provide accurate estimation of acutely infarcted nonhypertrophied myocardium. ${ }^{13,16,17}$ To our knowledge, this is also the first MRI study correlating the infarction size with LV function in hypertrophied hearts. It was demonstrated that infarction size was almost doubled in animals with cardiac hypertrophy in comparison to normal hearts, indicating the high sensitivity of hypertrophied hearts to ischemia. The complementary assessment of contrast enhancement and LV function using MRI provides information about the functional and structural changes in hypertrophied hearts subjected to ischemia.

Aortic blood pressure, measured proximal to the stenosis, was higher in animals with aortic banding compared to control. This finding confirms other published data. ${ }^{20,39}$ End-diastolic LV pressure was also higher in animals with aortic banding, which could be a sign of diastolic dysfunction resulting from cardiac hypertrophy and/or larger infarction size. ${ }^{40}$ These changes in hypertrophied hearts were associated with a decline in $\%$ wall thickening in remote myocardium and in positive and negative $\mathrm{dP} / \mathrm{dt}$. The larger infarction size, increased end-diastolic LV pressure, and EDV as well as decreased EF, positive and negative $\mathrm{dP} / \mathrm{dt}$, underscored the greater susceptibility of hypertrophied myocardium to ischemia.

In conclusion, hypertrophied hearts are more sensitive to ischemia than nonhypertrophied hearts, as shown in doubling of infarction size and malfunction of LV. The necrosis-specific contrast agent Gadophrin-3 allows accurate determination of infarct size in hypertrophied hearts. Thus, MRI is a suitable noninvasive diagnostic technique for assessment of LV mass, infarction size, and function of hypertrophied hearts.

\section{References}

1. Levy D, Garrison RJ, Savage DD, Kannel WB, Castelli WP. Prognostic implications of echocardiographically determined left ventricular mass in the Framingham Heart Study. N Engl J Med 1990; 322: 1561-1566.

2. Verdecchia P, Carini G, Circo A, Dovellini E, Giovannini E, Lombardo M, Solinas P, Gorini M, Maggioni AP. Left ventricular mass and cardiovascular morbidity in essential hypertension: the MAVI study. J Am Coll Cardiol 2001; 38: 1829-1835.

3. Sechtem U, Pflugfelder PW, Gould RG, Cassidy MM, Higgins CB. Measurement of right and left ventricular volumes in healthy individuals with cine MR imaging. Radiology 1987; 163: 697-702.

4. Lorenz CH, Walker ES, Morgan VL, Klein SS, Graham TP Jr. Normal human right and left ventricular mass, systolic function, and gender differences by cine magnetic resonance imaging. J Cardiovasc Magn Reson 1999; 1: 7-21. 
5. Schalla S, Nagel E, Lehmkuhl H, Klein C, Bornstedt A, Schnackenburg B, Schneider U, Fleck E. Comparison of magnetic resonance real-time imaging of left ventricular function with conventional magnetic resonance imaging and echocardiography. Am J Cardiol 2001; 87: 95-99.

6. Koyanagi S, Eastham CL, Harrison DG, Marcus ML. Increased size of myocardial infarction in dogs with chronic hypertension and left ventricular hypertrophy. Circ Res 1982; 50: 55-62.

7. Anderson PG, Allard MF, Thomas GD, Bishop SP, Digerness SB. Increased ischemic injury but decreased hypoxic injury in hypertrophied rat hearts. Circ Res 1990; 67: 948-959.

8. Laine GA. Microvascular changes in the heart during chronic arterial hypertension. Circ Res 1988; 62: 953-960.

9. Batra S, Rakusan K, Campbell SE. Geometry of capillary networks in hypertrophied rat heart. Microvasc Res 1991; 41: 29-40.

10. Conrad CH, Brooks WW, Hayes JA, Sen S, Robinson KG, Bing OH. Myocardial fibrosis and stiffness with hypertrophy and heart failure in the spontaneously hypertensive rat. Circulation 1995; 91: 161-170.

11. Wambolt RB, Lopaschuk GD, Brownsey RW, Allard MF. Dichloroacetate improves postischemic function of hypertrophied rat hearts. J Am Coll Cardiol 2000; 36: 1378-1385.

12. Lauerma K, Saeed M, Wendland MF, Derugin N, Yu KK, Higgins CB. The use of contrast-enhanced magnetic resonance imaging to define ischemic injury after reperfusion. Comparison in normal and hypertrophied hearts. Invest Radiol 1994; 29: 527-535.

13. Saeed M, Lund G, Wendland MF, Bremerich J, Weinmann H, Higgins CB. Magnetic resonance characterization of the peri-infarction zone of reperfused myocardial infarction with necrosis-specific and extracellular nonspecific contrast media. Circulation 2001; 103: 871-876.

14. Lund GK, Higgins CB, Wendland MF, Watzinger N, Weinmann HJ, Saeed M. Assessment of nicorandil therapy in ischemic myocardial injury by using contrast-enhanced and functional MR imaging. Radiology 2001; 221: 676-682.

15. Oshinski JN, Yang Z, Jones JR, Mata JF, French BA. Imaging time after Gd-DTPA injection is critical in using delayed enhancement to determine infarct size accurately with magnetic resonance imaging. Circulation 2001; 104: 2838-2842.

16. Pislaru SV, Ni Y, Pislaru C, Bosmans H, Miao Y, Bogaert J, Dymarkowski S, Semmler W, Marchal G, Van de Werf FJ. Noninvasive measurements of infarct size after thrombolysis with a necrosis-avid MRI contrast agent. Circulation 1999; 99: 690-696.

17. Barkhausen J, Ebert W, Debatin JF, Weinmann HJ. Imaging of myocardial infarction: comparison of magnevist and gadophrin-3 in rabbits. J Am Coll Cardiol 2002; 39: 1392-1398.

18. Marchal G, Ni Y, Herijgers P, Flameng W, Petre C, Bosmans H, Yu J, Ebert W, Hilger CS, Pfefferer D, Semmler W, Baert AL. Paramagnetic metalloporphyrins: infarct avid contrast agents for diagnosis of acute myocardial infarction by MRI. Eur Radiol 1996; 6: 2-8.

19. Hofmann B, Bogdanov A Jr, Marecos E, Ebert W, Semmler W, Weissleder R. Mechanism of gadophrin-2 accumulation in tumor necrosis. J Magn Reson Imag 1999; 9: 336-341.

20. Norton GR, Woodiwiss AJ, Gaasch WH, Mela T, Chung ES, Aurigemma GP, Meyer TE. Heart failure in pressure overload hypertrophy. The relative roles of ventricular remodeling and myocardial dysfunction. J Am Coll Cardiol 2002; 39: 664-671.

21. Chung ES, Perlini S, Aurigemma GP, Fenton RA, Dobson JG Jr, Meyer TE. Effects of chronic adenosine uptake blockade on adrenergic responsiveness and left ventricular chamber function in pressure overload hypertrophy in the rat. J Hypertens 1998; 16: 1813-1822.

22. Perings SM, Schulze K, Decking U, Kelm M, Strauer BE. Age-related decline of PCr/ATP-ratio in progressively hypertrophied hearts of spontaneously hypertensive rats. Heart Vessels 2000; 15: 197-202.

23. Reddy DS, Singh M, Ghosh S, Ganguly NK. Role of cardiac renin-angiotensin system in the development of pressure-overload left ventricular hypertrophy in rats with abdominal aortic constriction. Mol Cell Biochem 1996; 155: 1-11.

24. Harada K, Komuro I, Shiojima I, Hayashi D, Kudoh S, Mizuno T, Kijima K, Matsubara H, Sugaya T, Murakami K, Yazaki Y. Pressure overload induces cardiac hypertrophy in angiotensin II type $1 \mathrm{~A}$ receptor knockout mice. Circulation 1998; 97: 1952-1959. 
25. Hiramatsu M, Furukawa T, Sawanobori T, Hiraoka M. Ion channel remodeling in cardiac hypertrophy is prevented by blood pressure reduction without affecting heart weight increase in rats with abdominal aortic banding. J Cardiovasc Pharmacol 2002; 39: 866-874.

26. Sanada S, Node K, Asanuma H, Ogita H, Takashima S, Minamino T, Asakura M, Liao Y, Ogai A, Kim J, Hori M, Kitakaze M. Opening of the adenosine triphosphate-sensitive potassium channel attenuates cardiac remodeling induced by long-term inhibition of nitric oxide synthesis: role of 70-kDa S6 kinase and extracellular signal-regulated kinase. J Am Coll Cardiol 2002; 40: 991-997.

27. Myerson SG, Montgomery HE, World MJ, Pennell DJ. Left ventricular mass: reliability of M-mode and 2dimensional echocardiographic formulas. Hypertension 2002; 40: 673-678.

28. Bottini PB, Carr AA, Prisant LM, Flickinger FW, Allison JD, Gottdiener JS. Magnetic resonance imaging compared to echocardiography to assess left ventricular mass in the hypertensive patient. Am J Hypertens 1995; 8: 221-228.

29. Schwitter J, Nanz D, Kneifel S, Bertschinger K, Buchi M, Knusel PR, Marincek B, Luscher TF, von Schulthess GK. Assessment of myocardial perfusion in coronary artery disease by magnetic resonance: a comparison with positron emission tomography and coronary angiography. Circulation 2001; 103: 2230-2235.

30. Nagel E, Lehmkuhl HB, Bocksch W, Klein C, Vogel U, Frantz E, Ellmer A, Dreysse S, Fleck E. Noninvasive diagnosis of ischemia-induced wall motion abnormalities with the use of high-dose dobutamine stress MRI: comparison with dobutamine stress echocardiography. Circulation 1999; 99: 763-770.

31. Klein C, Nekolla SG, Bengel FM, Momose M, Sammer A, Haas F, Schnackenburg B, Delius W, Mudra H, Wolfram D, Schwaiger M. Assessment of myocardial viability with contrast-enhanced magnetic resonance imaging: comparison with positron emission tomography. Circulation 2002; 105: 162-167.

32. Ni Y, Pislaru C, Bosmans H, Pislaru S, Miao Y, Bogaert J, Dymarkowski S, Yu J, Semmler W, Van de Werf F, Baert AL, Marchal G. Intracoronary delivery of Gd-DTPA and Gadophrin-2 for determination of myocardial viability with MR imaging. Eur Radiol 2001; 11: 876-883.

33. Jeong AK, Choi SI, Kim DH, Park SB, Lee SS, Choi SH, Lim TH. Evaluation by contrast-enhanced MR imaging of the lateral border zone in reperfused myocardial infarction in a cat model. Korean J Radiol 2001; 2: 2127.

34. Maxwell MP, Hearse DJ, Yellon DM. Species variation in the coronary collateral circulation during regional myocardial ischaemia: a critical determinant of the rate of evolution and extent of myocardial infarction. Cardiovasc Res 1987; 21: 737-746.

35. Dellsperger KC, Clothier JL, Koyanagi S, Inou T, Marcus ML. Effects of coronary artery occlusion in animals with hypertension and left ventricular hypertrophy. J Cardiovasc Pharmacol 1991; 17: S40-45.

36. Russell RR 3rd, Yin R, Caplan MJ, Hu X, Ren J, Shulman GI, Sinusas AJ, Young LH. Additive effects of hyperinsulinemia and ischemia on myocardial GLUT1 and GLUT4 translocation in vivo. Circulation 1998; 98 : 2180-2186.

37. Gaasch WH, Zile MR, Hoshino PK, Weinberg EO, Rhodes DR, Apstein CS. Tolerance of the hypertrophic heart to ischemia. Studies in compensated and failing dog hearts with pressure overload hypertrophy. Circulation 1990; 81: 1644-1653.

38. Takeuchi K, Buenaventura P, Cao-Danh H, Glynn P, Simplaceanu E, McGowan FX, del Nido PJ. Improved protection of the hypertrophied left ventricle by histidine-containing cardioplegia. Circulation 1995; 92: II395-399.

39. Marano G, Palazzesi S, Fadda A, Vergari A, Ferrari AU. Attenuation of aortic banding-induced cardiac hypertrophy by propranolol is independent of beta-adrenoceptor blockade. J Hypertens 2002; 20: 763769.

40. Sia YT, Lapointe N, Parker TG, Tsoporis JN, Deschepper CF, Calderone A, Pourdjabbar A, Jasmin JF, Sarrazin JF, Liu P, Adam A, Butany J, Rouleau JL. Beneficial effects of long-term use of the antioxidant probucol in heart failure in the rat. Circulation 2002; 105: 2549-2555. 

Effect of potassium-channel opener therapy on reperfused infarction in hypertrophied hearts: Demonstration of preconditioning by using functional and contrast-enhanced magnetic resonance imaging 


\section{Abstract}

Background: Effects of therapy with the potassium-channel opener and vasodilator nicorandil were studied in reperfused infarction of hypertrophied hearts using magnetic resonance imaging (MRI), hemodynamic measurements and histochemical staining.

Methods: Twenty-two Sprague Dawley rats were subjected to aortic banding to induce left ventricular hypertrophy (LVH); 11 served as control. Eight weeks later, the left coronary artery was occluded ( $25 \mathrm{~min}$ ) in all 33 animals followed by $3 \mathrm{~h}$ reperfusion. During occlusion 11 rats with LVH received nicorandil $(0.1 \mathrm{mg} / \mathrm{kg}$ bolus and $1.5 \mathrm{mg} / \mathrm{kg} / \mathrm{h}$ for $3 \mathrm{~h})$. The new necrosis-specific contrast agent Gadophrin-3 was administered to all animals to delineate infarction on multislice T1-weighted spin echo MR images.

Results: Nicorandil increased ischemic tolerance of LVH as shown by the reduction of infarction size from $19.3 \pm 1.3 \%$ to $10.0 \pm 2.5 \%$ LV $(p=0.005)$. Infarction size in treated animals was identical to control $(9.3 \pm 1.6 \%)$. A close correlation was found between MRI and postmortem findings. Functional MRI revealed improvement in ejection fraction in nicorandil-treated hearts $(48.5 \pm 3.4 \%$ vs. $38.1 \pm 3.2 \%, p=0.04)$. LV end-diastolic volume and pressure, aortic pressure and peripheral vascular resistance were highest in untreated hypertrophied hearts.

Conclusions: Brief ischemia caused severe injury in hypertrophied hearts. Infusing nicorandil increased the tolerance of hypertrophied hearts to ischemia. MRI is a suitable technique for the evaluation of new therapies in LVH. 


\section{Introduction}

Left ventricular (LV) hypertrophy associated with chronic arterial hypertension is a strong predictor of myocardial infarction, congestive heart failure and sudden death. ${ }^{1}$ Early studies have demonstrated the high sensitivity of the hypertrophied heart to ischemia. ${ }^{2,3}$ Therefore, therapeutic strategies to reduce infarction size in hypertrophied hearts are important. In ischemic non-hypertrophied hearts, the potassium-channel opener nicorandil preserves myocardial viability and microvascular integrity. ${ }^{4}$ Nicorandil is a cardioprotective agent with both nitrate-like and adenosine triphosphate-sensitive potassium-channel $\left(\mathrm{K}_{\text {ATP }}\right)$ activating properties. ${ }^{5,6}$ Activation of the $\mathrm{K}_{\text {ATP }}$-channel leads to dilation of resistance arteries whereas nitrate moieties dilate capacitance veins. However, the sensitivity of these target sites may change during cardiac hypertrophy.

Assessment of new therapies designed to reduce infarction size needs accurate and reproducible diagnostic methods. Furthermore, early and accurate measurements of left ventricular (LV) mass, function and infarction size are crucial in patients with LV hypertrophy. Magnetic resonance (MR) imaging with necrosis-specific contrast media provides an accurate estimation of acutely infarcted myocardium. ${ }^{7,8}$ In addition, anatomic and functional parameters, such as LV mass, volumes and regional and global myocardial function, can be obtained with MR imaging. ${ }^{9-11}$ To our knowledge, the effects of nicorandil and Gadophrin-3 have not been studied in ischemically injured hypertrophied hearts. Thus, the aim of this study was to examine the effects of nicorandil on ischemically injured hypertrophied hearts using functional and contrast-enhanced MR imaging, hemodynamic parameters and postmortem histochemical staining.

\section{Methods}

\section{Animal preparation}

The experimental protocol was approved by the Committee on Animal Research at this institution and confirms with the National Institutes of Health guidelines for care and use of laboratory animals. Sprague Dawley rats ( $n=33$, female, 90-110g; Simonson Laboratories, Modesto, CA) were assigned to 3 groups: Control, untreated myocardial hypertrophy and nicorandil-treated myocardial hypertrophy ( $\mathrm{n}=11$ rats per group). To induce myocardial hypertrophy in the last 2 groups, aortic banding was performed 8 weeks prior to MR imaging. Rats were anesthetized by intraperitoneally injecting ketamine $(50 \mathrm{mg} / \mathrm{kg}$, Ketaset, Fort Dodge Inc., Fort Dodge, lowa) and xylazine $(1.4 \mathrm{mg} / \mathrm{kg}$, Xyla-Ject, Phoenix Inc., St. Joseph, MO). After incision at the left flank, the aorta was isolated. A steel wire $(0.5 \mathrm{~mm}$ diameter $)$ was placed close to the surface of the suprarenal aorta. A thread was passed around both the aorta and wire and tied to produce aortic stenosis. Temporary closure of the abdominal aorta was confirmed by a red to 
pale color change of the kidneys. The wire was then removed and the color of the kidney returned to red. The abdominal muscle wall and skin were subsequently closed. All rats were kept under the same controlled conditions and given food and water ad libitum.

Eight weeks later, all animals were anesthetized (sodium pentobarbital, 50mg/kg; Nembutal Sodium Solution, Abbot Laboratories, North Chicago, IL). After tracheostomy, animals were mechanically ventilated and subjected to left thoracotomy. The proximal portion of the left coronary artery was occluded for $25 \mathrm{~min}$ with a snare around the coronary artery to produce infarction, followed by 3 hrs reperfusion prior to imaging.

Nicorandil (Chugai Pharmaceutical Co, Ltd, Tokyo, Japan) was administered intravenously $(0.1 \mathrm{mg} / \mathrm{kg}$ bolus followed by an infusion of $1.5 \mathrm{mg} / \mathrm{kg} / \mathrm{hr}$ for 3 hours) at $15 \mathrm{~min}$ of coronary occlusion in the treatment group. Gadophrin-3 (\{mu-[\{16,16'-[(7,12-Diethyl3,8,13,17-tetramethyl-21H,23H-porphine-2,18-diyl-N21,N22,N23,N24)copper]bis[3,6,9tris(carboxymethyl)11,14-dioxo-3,6,9,12,13-pentaazahexadecanoato]\}(8-)]\}digadolinate (2-), disodium, Schering AG, Berlin, Germany), a porphyrin based T1-enhancing gadolinium agent, was administered intravenously $(0.05 \mathrm{mmol} / \mathrm{kg})$ in all animals 5 minutes after the start of reperfusion. Gadophrin-3 is a necrosis-specific metalloporphyrin that binds to necrotic tissue or cellular debris ${ }^{12}$ and produces a sustained contrast between normal and necrotic tissue that may last for more than 24 hours. Physicochemical and pharmacological properties of Gadophrin-3 are comparable to those of Gadophrin-2 with $\mathrm{T} 1$ and $\mathrm{T} 2$ relaxivities of 8.9 and $12 \mathrm{sec}^{-1} \mathrm{mM}^{-1}$, but improved stability owing to the addition of a copper atom to the center of the porphyrin molecule. ${ }^{13}$

\section{Magnetic resonance imaging}

Three hours after the Gadophrin-3 injection, MR imaging was performed. Animals were placed supine in a $5.0 \mathrm{~cm}$ diameter birdcage receiver-transmitter coil. Each animal was connected to an ECG-monitor that allowed monitoring of heart rate and provided trigger signals at the $\mathrm{R}$ waves for cardiac gating of the MR imaging sequence. MR images were acquired using a 2.0 Tesla system (Omega CSI, Bruker Instruments, Fremont, CA) equipped with self-shielded gradient coils ( \pm 20 gauss $/ \mathrm{cm}, 15 \mathrm{~cm}$ bore size).

Multislice T1-weighted spin echo imaging was used to measure the size of infarction 3 hours after administration of contrast media. Acquisition parameters were repetition time, $T R=300$, echo time, $T E=12 \mathrm{~ms}$, matrix $=256 \times 128$ data points interpolated to $256 \times 256$ during Fourier transformation, field of view $=50 \times 50 \mathrm{~mm}$, acquisition time $=2.5$ min. The entire heart was covered at end-diastole and end-systole (repetition time, $\mathrm{TR}=45 \%$ of each $\mathrm{R}-\mathrm{R}$ interval) in short axis views using $2 \mathrm{~mm}$ slice thickness. 


\section{MR data analysis}

The MR images were analyzed with the NIH Image software version 1.59 (NIH, Bethesda, MD). By delineating LV boundaries on end-diastolic and end-systolic images and adding them together, LV volumes, mass and ejection fraction were determined. The region of the Gadophrin-enhanced zone was delineated on end-diastolic contrastenhanced images to determine infarction size. The extent of the infarcted region, defined as a percentage of the total LV, was calculated as the sum of the Gadophrinenhanced regions for all slices divided by the sum of the LV cross-sectional areas from all slices. Wall thickness [mm] and systolic thickening [\%] were measured on 2 contiguous mid-ventricular slices in the center of the Gadophrin-enhanced zone (antero-lateral wall) and in remote myocardium.

\section{Hemodynamic measurements}

Aortic and LV pressures as well as maximum rate of rise and decline of ventricular pressure (positive and negative $\mathrm{dP} / \mathrm{dt}$ ) were measured after MR imaging. A catheter was therefore introduced via the right carotid artery into the aortic arch and the LV. Stroke volume (SV) in $\mathrm{ml}$ was calculated from MR data as end-diastolic volume minus endsystolic volume (EDV-ESV). Cardiac output in $\mathrm{ml} / \mathrm{min}$ was calculated as SV multiplied by heart rate, and systemic vascular resistance in $\mathrm{mmHg} / \mathrm{l} / \mathrm{min}$ as mean aortic pressure divided by cardiac output.

\section{Postmortem evaluation}

At the completion of the MR imaging and hemodynamic measurement protocol, the left coronary artery was re-occluded and $0.2 \mathrm{ml}$ of phthalocyanine blue dye injected into a tail vein. This dye imparts a blue color to normally perfused myocardium, while the territory of the occluded artery (area at risk) remains unstained. The heart was excised 3 minutes post-injection. The atria and right ventricle were removed and the LV was weighed.

The LV was transversely sliced into $2 \mathrm{~mm}$ thick slices to correspond to the location of MR images. The slices were incubated in $2 \%$ triphenyl-tetrazolium-chloride-solution (TTC) for 8 minutes at $38^{\circ} \mathrm{C}$ to delineate viable myocardium. Viable myocardium appeared brick red while infarcted regions were not stained. Both upper and lower surfaces of the stained ventricular slices were digitally photographed and analyzed to determine the size of area at risk and of the infarction. 


\section{Statistical analysis}

Data were expressed as mean \pm standard error of the mean. The unpaired Student t-test was used to compare control non-hypertrophied, untreated hypertrophied and nicorandil treated groups. A $p<0.05$ was considered statistically significant. Linear regression analysis was employed to determine the correlation between postmortem and MR measurements.

\section{Results}

Infarction size in hypertrophied and non-hypertrophied hearts

Figure 1 shows MR images acquired from the entire hearts of untreated hypertrophied and nicorandil-treated hypertrophied hearts. It clearly demonstrates the differences in the infarction size between the 2 groups. On Gadophrin-3 enhanced MR images, the size of infarction was significantly larger in untreated hypertrophied hearts $(19.3 \pm 1.3 \%$ LV) than non-hypertrophied control $(9.3 \pm 1.6 \% \mathrm{LV}, \mathrm{p}=0.01)$ and nicorandil-treated hypertrophied hearts $(10.0 \pm 2.5 \% \mathrm{LV}, \mathrm{p}=0.005$ vs. untreated hypertrophied hearts, $p=0.8$ vs. control) (Figure 2). However, there was no significant difference in infarction size between non-hypertrophied control and nicorandil-treated hypertrophied hearts (Table 1).

The infarction size determined after administration of Gadophrin-3 was similar to that measured at postmortem (Figure 3) (regression analysis for control: $r=0.99$, standard error of the estimate $(S E E)= \pm 0.45$, regression line equation $y=1.06 x-0.36$; hypertrophy: $r=0.95, \quad S E E= \pm 1.40, y=0.89 x+2.69$; nicorandil-treated hypertrophy: $r=0.99$, $\mathrm{SEE}= \pm 0.57, y=0.99 x+0.24)$. Figure 4 shows the correlation between the Gadophrinenhanced regions on MR imaging and TTC-negative regions on histochemical staining in the three groups. No differences in areas at risk for infarction were present between the groups (control: $37.0 \pm 1.3 \% \mathrm{LV}$; non-treated hypertrophied hearts: $38.4 \pm 1.3 \% \mathrm{LV}$; nicorandil-treated hypertrophied hearts: $38.1 \pm 1.9 \% \mathrm{LV}, \mathrm{p}=0.66$ versus control and 0.91 versus non-treated hypertrophy).

\section{LV mass}

The determination of LV mass from MR images agreed closely with the mass determined at postmortem (regression analysis for control: $r=0.97, S E E= \pm 0.02, y=1.11 x-0.07$; hypertrophy: $r=0.89, S E E= \pm 0.02, y=0.95 x+0.03$; nicorandil-treated hypertrophy: $r=0.98$, $\mathrm{SEE}= \pm 0.02, y=1.08 \mathrm{x}-0.08$ ) (Figure 5 ). An increase in LV mass was found in rats subjected to aortic banding compared to control (Table 1). LV mass in the rats subjected to aortic banding was identical in the treated and non-treated group ( $p=0.40$ for MR imaging, $p=0.45$ for postmortem). The increase in LV mass was not attributed to differences in 
body weight, because no significant differences were present between the three groups (Table 1).
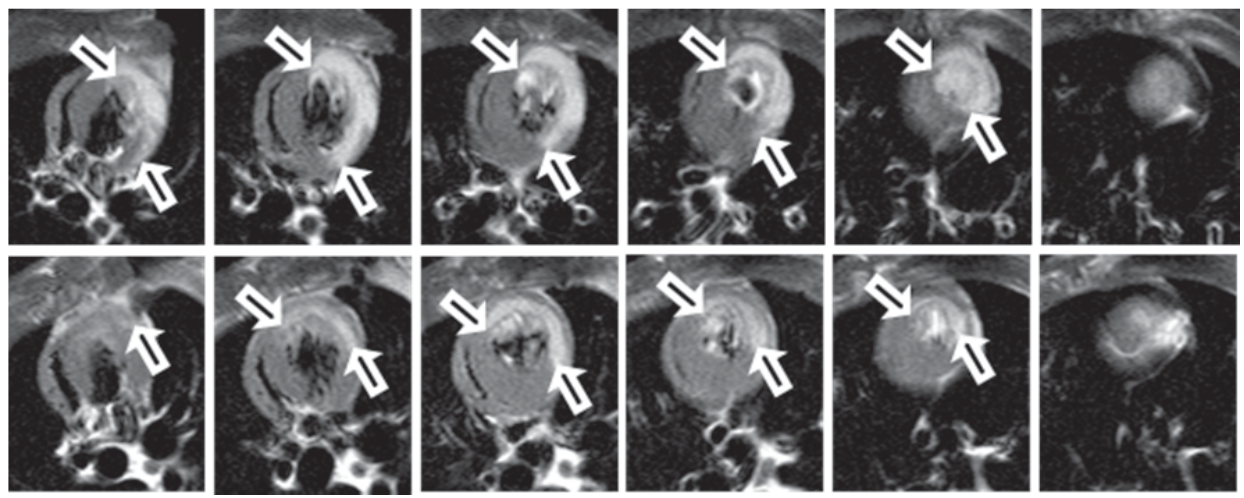

Figure 1: Multislice Gadophrin-3-enhanced T1-weighted spin echo MR images of rat hearts (short axis view) subjected to $25 \mathrm{~min}$ ischemia and $3 \mathrm{~h}$ of reperfusion.

Top row: Untreated animal with hypertrophied heart.

Bottom row: Animal with hypertrophied heart treated with intravenous infusion of nicorandil initiated $15 \mathrm{~min}$ after coronary artery occlusion and continued for 3 hours during reperfusion. Infarction size is indicated by arrows. The extent of infarction in the untreated hypertrophied heart is larger, more transmural and involves one more slice in comparison with the nicorandil-treated hypertrophied heart.

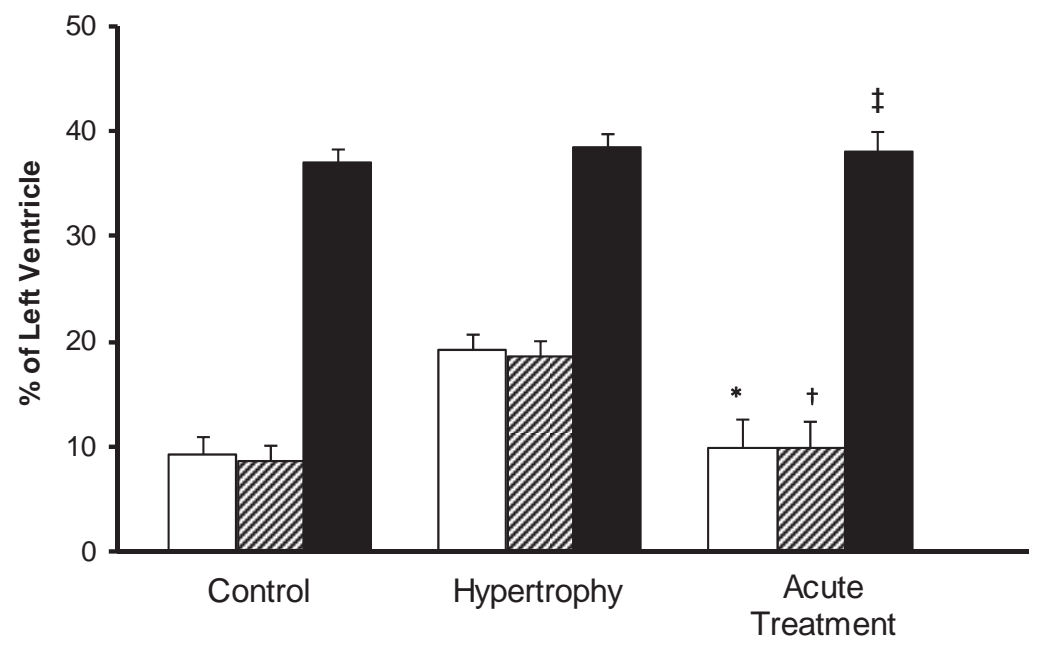

Figure 2: Bar graph of infarction size as \% of left ventricular area (\% LV) in control, hypertrophied untreated and acutely nicorandil-treated hypertrophied myocardium determined with MR imaging (white column), postmortem TTC staining (gray) and area at risk determined with postmortem blue dye staining (black). Infarction size was larger in hypertrophied myocardium while control and acutely treated hypertrophied hearts showed similar infarction size. Areas at risk were not different between the groups.

${ }^{*} p=0.80$ versus control, $p=0.005$ versus hypertrophy

$+p=0.69$ versus control, $p=0.008$ versus hypertrophy 
Table 1: MR and postmortem data obtained from control (non-hypertrophied), untreated hypertrophied and nicorandil-treated hypertrophied heart groups subjected to $25 \mathrm{~min}$ ischemia and $3 \mathrm{~h}$ reperfusion.

\begin{tabular}{lccc}
\hline & Control & $\begin{array}{c}\text { Untreated } \\
\text { hypertrophied hearts }\end{array}$ & $\begin{array}{c}\text { Nicorandil-treated } \\
\text { hypertrophied hearts }\end{array}$ \\
\hline Body weight, g & $245 \pm 6$ & $248 \pm 5$ & $247 \pm 7$ \\
MR LV mass, g & $0.61 \pm 0.02$ & $0.81 \pm 0.01^{*}$ & $0.77 \pm 0.04^{*}$ \\
Postmortem LV mass, g & $0.61 \pm 0.02$ & $0.82 \pm 0.01^{*}$ & $0.79 \pm 0.03^{*}$ \\
MRI infarction, \%LV & $9.3 \pm 1.6$ & $19.3 \pm 1.3^{*}$ & $10.0 \pm 2.5^{+}$ \\
PM infarction, \%LV & $8.6 \pm 1.6$ & $18.7 \pm 1.4^{*}$ & $9.9 \pm 2.5^{\dagger}$ \\
Area at risk, \%LV & $37.0 \pm 1.3$ & $38.4 \pm 1.3$ & $38.1 \pm 1.9$ \\
Wall thickness remote, mm & $2.33 \pm 0.08$ & $2.88 \pm 0.10^{*}$ & $3.34 \pm 0.07^{*}{ }^{+}$ \\
Wall thickness infarction, $\mathrm{mm}$ & $2.39 \pm 0.06$ & $2.93 \pm 0.10^{*}$ & $3.12 \pm 0.10^{*}$ \\
WT remote, \% & $35.5 \pm 1.7$ & $27.9 \pm 2.3^{*}$ & $30.2 \pm 0.8^{*}$ \\
WT infarction, \% & $3.7 \pm 0.8$ & $4.1 \pm 2.2$ & $4.0 \pm 0.7$ \\
\hline
\end{tabular}

Mean \pm standard error of the mean. MR LV mass denotes left ventricular mass determined on MR images; MRI infarction, Gadophrin-enhanced area on magnetic resonance images; PM infarction, infarction area defined at postmortem by triphenyl-tetrazolium-chloride stain; Area at risk, areas at risk for infarction defined by blue dye stain; Wall thickness, diastolic wall thickness in remote and infarcted region; \%WT, percent systolic wall thickening.; ${ }^{*} p<0.05$ compared to control non-hypertrophied hearts.; ${ }^{\dagger} \mathrm{p}<0.05$ compared to untreated hypertrophied hearts.

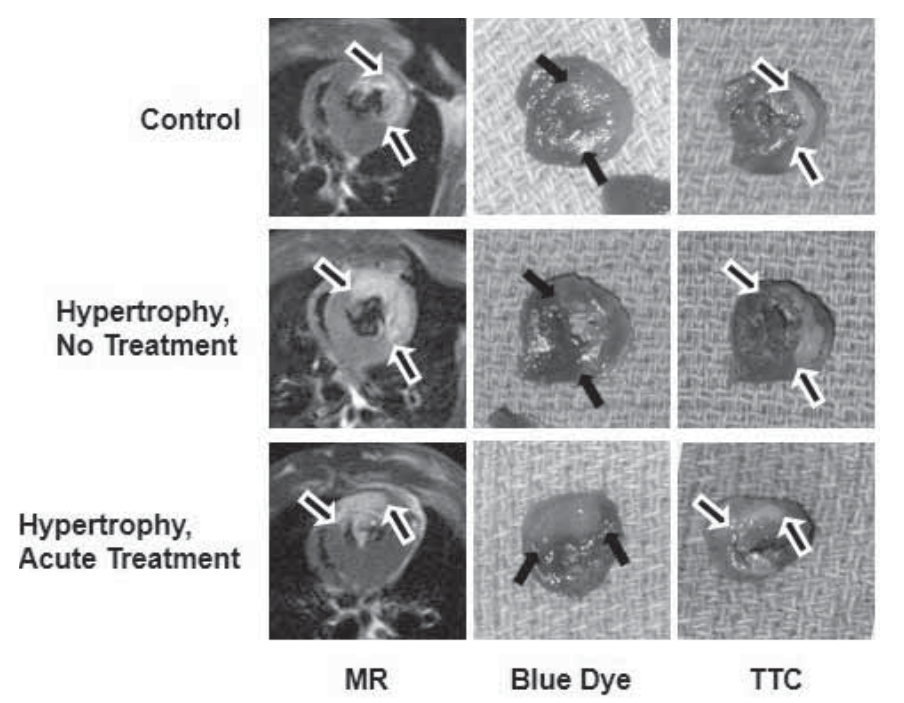

Figure 3: Extent of Gadophrin-3-enhanced region on MR images (left), area at risk after phthalocyanine bluedye staining (middle) and true infarction size after triphenyl-tetrazolium-chloride (TTC) staining at postmortem (right) in control (top row), hypertrophied rat heart without treatment (middle row) and hypertrophied rat heart with acute nicorandil treatment (bottom row). Contrast-enhanced region and true infarction size were larger in hypertrophied hearts while these areas were similar in control and treated hypertrophied hearts. Areas at risk (black arrows) were similar in all groups. 
Control: Infarction Size

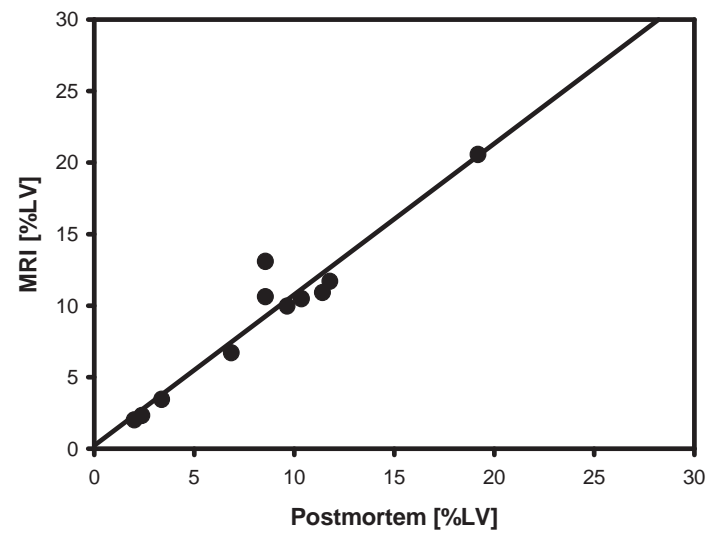

Hypertrophy: Infarction Size

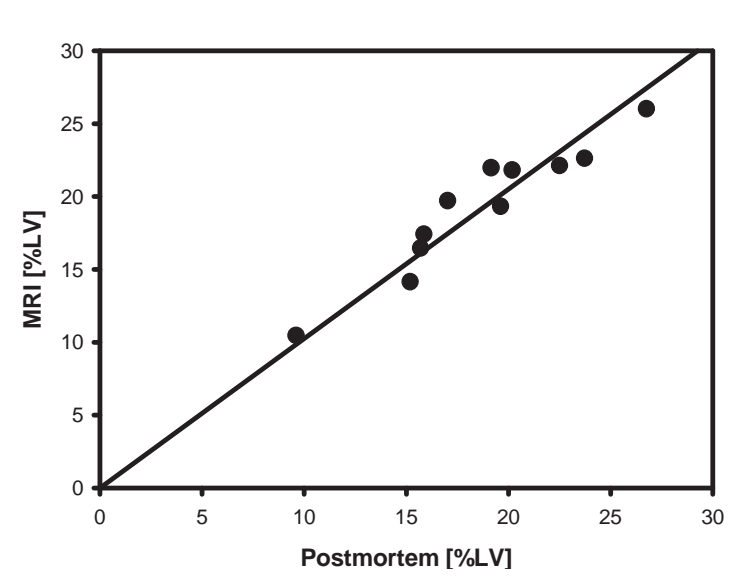

Acute Treatment: Infarction Size

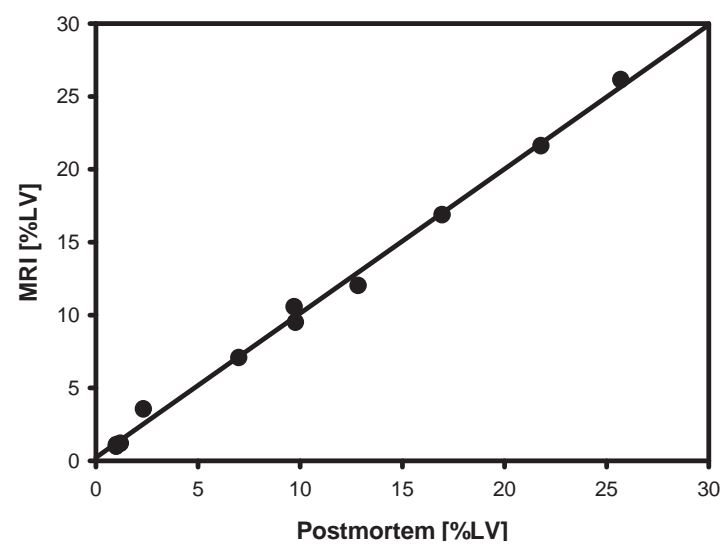

Postmortem [\%LV]
Figure 4: Scatterplots and regression line of infarction size determined with magnetic resonance imaging (MRI) and postmortem histochemical staining (Postmortem) for control non-hypertrophied (top), hypertrophied untreated (middle) and acutely nicorandil-treated hypertrophied hearts (bottom). [\% LV] denotes percentage of left ventricle in which enhancement or staining was observed. 


\section{Control: LV Mass}

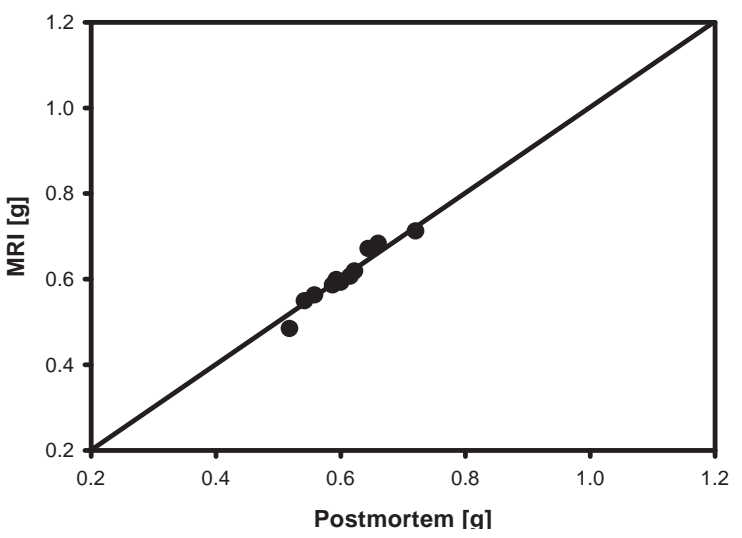

Hypertrophy: LV Mass

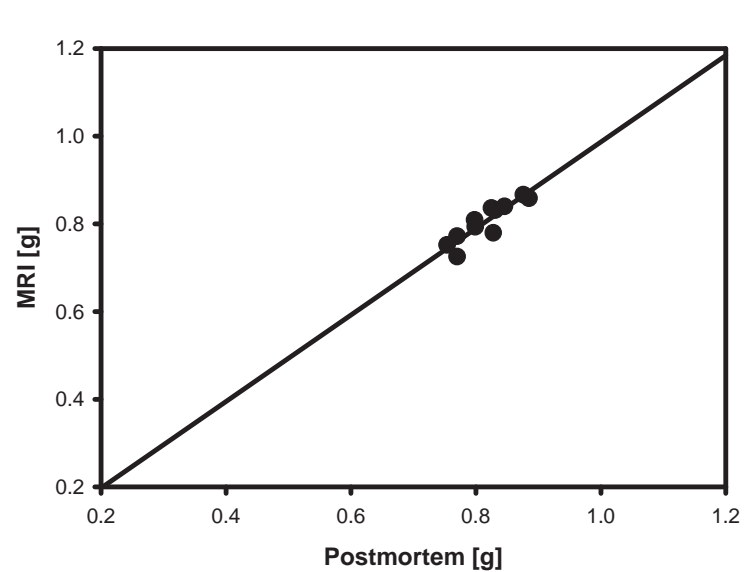

Acute Treatment: LV Mass

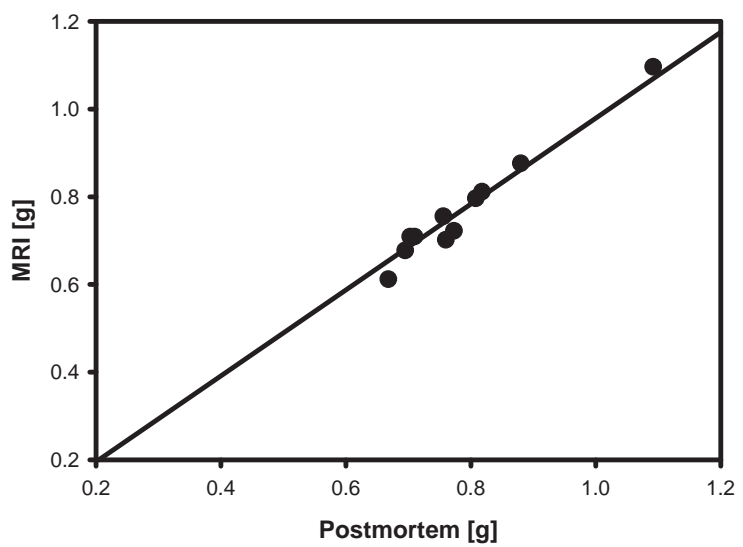

Postmortem [g]
Figure 5: Scatterplots and regression line of left ventricular mass determined with magnetic resonance imaging (MRI) and at postmortem (Postmortem) for control non-hypertrophied (top), hypertrophied untreated and (middle) and acutely nicorandil-treated hypertrophied hearts (bottom). 


\section{LV function}

Nicorandil infusion prevented the dilatation of the hypertrophied hearts after coronary occlusion-reperfusion and the decline in ejection fraction (EF) (Table 2). Ischemically injured untreated hypertrophied hearts showed larger LV end-diastolic volumes than non-hypertrophied control $(p=0.012)$ and nicorandil-treated hypertrophied hearts $(p=0.025)$ (Table 2). Hypertrophied hearts showed a significant reduction in ejection fraction compared to non-hypertrophied control $(p=0.007)$ and treated hypertrophied hearts $(p=0.04)$. These changes can most likely be attributed to the larger infarction size seen in untreated hypertrophied hearts.

Representative MR images acquired during systole and diastole from control nonhypertrophied, untreated hypertrophied, and nicorandil-treated hypertrophied hearts are shown in Figure 6. The diastolic LV wall thickness in remote and infarcted myocardium of nicorandil-treated and untreated hypertrophied hearts was significantly larger than that of control non-hypertrophied hearts (Table 1). In addition, wall thickness in remote myocardium in nicorandil-treated hypertrophied hearts was significantly larger than in untreated hypertrophied hearts. The thinner LV wall in untreated hypertrophied hearts is in line with larger EDV and lower EF as a sign of LV dilation.

Myocardial wall thickening (\% systolic wall thickening) in remote regions was less in both treated $(p=0.014)$ and untreated hypertrophied hearts $(p=0.020)$ compared to control (Table 1). No differences were seen in infarcted regions between the 3 groups.

Table 2: Hemodynamic parameters obtained from control (non-hypertrophied), untreated hypertrophied and nicorandil-treated hypertrophied heart animal groups subjected to $25 \mathrm{~min}$ ischemia and $3 \mathrm{~h}$ reperfusion.

\begin{tabular}{|c|c|c|c|}
\hline & Control & $\begin{array}{l}\text { Untreated hypertrophied } \\
\text { hearts }\end{array}$ & $\begin{array}{l}\text { Nicorandil-treated } \\
\text { hypertrophied hearts }\end{array}$ \\
\hline $\mathrm{HR}$, beats/min & $382 \pm 9$ & $358 \pm 12$ & $361 \pm 11$ \\
\hline Mean BP, mmHg & $79 \pm 3$ & $108 \pm 2 *$ & $98 \pm 2 *^{*}$ \\
\hline LVEDP, $\mathrm{mmHg}$ & $4 \pm 1$ & $13 \pm 1^{*}$ & $8 \pm 1^{* *^{+}}$ \\
\hline$+d P / d t$ & $9300 \pm 652$ & $4600 \pm 400^{*}$ & $7100 \pm 507^{*}{ }^{+}$ \\
\hline$-d P / d t$ & $-8700 \pm 575$ & $-4433 \pm 348 *$ & $-6300 \pm 435^{*}{ }^{\dagger}$ \\
\hline $\mathrm{EDV}, \mathrm{ml}$ & $0.16 \pm 0.01$ & $0.21 \pm 0.02 *$ & $0.18 \pm 0.01^{\dagger}$ \\
\hline$E F, \%$ & $49.2 \pm 1.6$ & $38.1 \pm 3.2^{*}$ & $48.5 \pm 3.4^{+}$ \\
\hline $\mathrm{CO}, \mathrm{ml} / \mathrm{min}$ & $30.7 \pm 1.5$ & $28.7 \pm 2.7$ & $31.2 \pm 3.3$ \\
\hline $\mathrm{PVR}, \mathrm{mmHg} / \mathrm{l} / \mathrm{min}$ & $2647 \pm 182$ & $3712 \pm 96 *$ & $3082 \pm 314$ \\
\hline
\end{tabular}

Mean \pm standard error of the mean. $H R=$ heart rate; Mean $B P=$ mean aortic blood pressure. LVEDP=left ventricular end-diastolic pressure; $+/-\mathrm{dP} / \mathrm{dt}$, positive and negative $\mathrm{dP} / \mathrm{dt}$; $\mathrm{EDV}=\mathrm{end}$-diastolic volume; $E F=e j e c t i o n$ fraction; $C O=$ cardiac output; $P V R=$ peripheral vascular resistance.; ${ }^{*} p<0.05$ compared to control non-hypertrophied hearts.; ${ }^{\dagger} p<0.05$ compared to untreated hypertrophied hearts. 


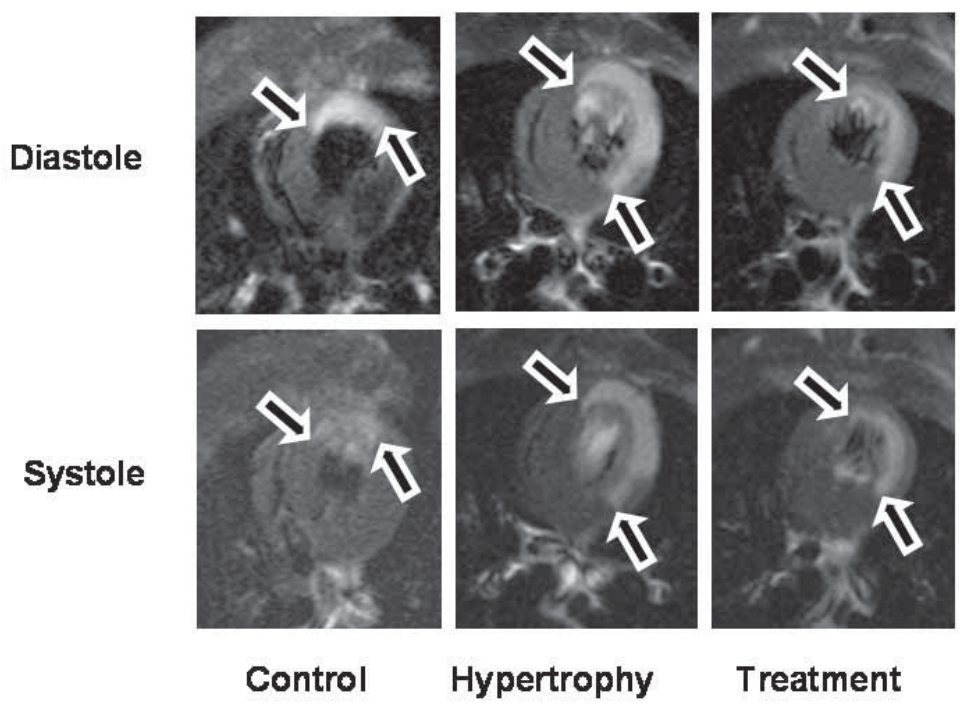

Figure 6: Gadophrin-3-enhanced T1-weighted spin echo MR images of rat hearts in short axis view in end-diastole (top row) and end-systole (bottom row) subjected to 25 minutes of ischemia and 3 hours of reperfusion. Left: control animal without cardiac hypertrophy; middle: untreated animal with cardiac hypertrophy; right: animal with cardiac hypertrophy that was acutely treated with an intravenous infusion of nicorandil initiated 15 minutes after coronary artery occlusion and continued for 3 hours during reperfusion.

\section{Hemodynamic measurements}

Hemodynamic measurements and derived values are given in Table 2. Heart rate and cardiac output were similar in all 3 groups. Mean aortic blood pressure was different between non-hypertrophied control and untreated hypertrophied hearts $(p=0.003)$. Infusion of nicorandil significantly lowered blood pressure $(p=0.012)$ in the treatment group compared with the untreated hypertrophied hearts. LV end-diastolic pressure and values of $+\mathrm{dP} / \mathrm{dt}$ and $-\mathrm{dP} / \mathrm{dt}$ for treated hypertrophied hearts were between those of the control and untreated hypertrophied hearts. EDV and LV end-diastolic pressure were highest in untreated animals with aortic banding.

\section{Discussion}

The main findings of this study are that 1 ) brief coronary occlusion produces large infarction in hypertrophied hearts compared to non-hypertrophied hearts, 2) nicorandil treatment reduces infarction size in hypertrophied hearts to the size seen in control non-hypertrophied hearts and preserves left ventricular function, and 3) MR imaging 
allows accurate measurements of LV mass, function and after administration of Gadophrin-3, of infarction size. Thus, it is a suitable diagnostic tool to monitor the acute changes in ischemic LV hypertrophy and the effects of new therapies designed to protect hypertrophied hearts from ischemia.

Nicorandil is a hybrid cardiovascular agent with $\mathrm{K}_{\text {ATP }}$ channel opener and nitrate properties. ${ }^{5}$ It is approved for clinical use as an antianginal drug in Europe and Japan. ${ }^{14}$ Ischemic tolerance during coronary angioplasty was improved and major coronary events in patients with stable angina were reduced. ${ }^{4-6}$

Nicorandil can activate the sarcolemmal and mitochondrial $K_{\text {ATP-Channels and also }}$ release nitric oxide. ${ }^{15}$ The opening of the mitochondrial $K_{\text {ATP-channels alters the redox }}$ state of cardiomyocytes and prevents mitochondial $\mathrm{Ca}^{2+}$ overload, whereas the opening

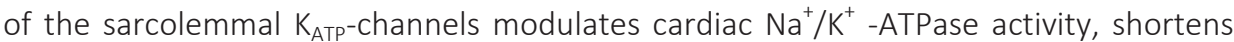
the action potential duration, and also prevents intracellular $\mathrm{Ca}^{2+}$ overload. ${ }^{16,17}$ Sakai et al. reported a subcellular localization of nicorandil in cardiomyocyte mitochondria of rats, where nicorandil is partially transformed into denitrated nicorandil with the release of nitric oxide. ${ }^{18}$ Nitric oxide has been implicated as a mediator of ischemic pre-

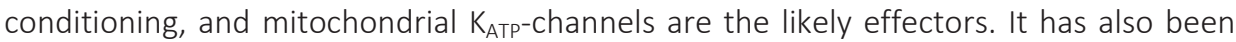
postulated that intracellular calcium overloading in the mitochondria decreases ATPproduction, contributing to myocardial stunning and subsequent cellular damage. ${ }^{19}$

At the arteriolar level, nicorandil, via the release of nitric oxide, activates the enzyme guanylate cyclase which in turn induces an increase in intracellular cGMP and a decrease in intracellular calcium. ${ }^{20}$ Other potential effects of nicorandil are an increase in coronary blood flow, a reduction in mean arterial blood pressure, in pre- and afterload, and several others, such as acting as a free radical scavenger and neutrophil modulating agent. ${ }^{6,21-23}$

In nonhypertrophied rat hearts subjected to reperfused infarction of 45 minutes, treatment with nicorandil suppressed microvascular permeability, reduced infarction size and improved left ventricular function. ${ }^{24}$ In rabbits subjected to 20 to 30 minutes of reperfused myocardial infarction, a decrease of infarction size and incidence of arrhythmias, as well as an increase in the survival rate, was observed in the nicorandil treatment group. ${ }^{25}$ Treatment with nicorandil also reduced infarction size in dogs in which myocardial ischemia was induced. ${ }^{15,22}$

Matsuo et al. demonstrated a cardioprotective effect of intravenously administered nicorandil during angioplasty independent of collateral flow to the ischemic site in patients with proximal stenosis of the left anterior descending coronary artery. ${ }^{26}$ Ito et al. found an improved LV function in patients with anterior wall infarction treated with nicorandil before coronary angioplasty in comparison with the placebo group. ${ }^{4}$ The efficacy of nicorandil in addition to conventional antianginal drugs was evaluated in a large, randomised study. ${ }^{6}$ The primary endpoint of myocardial infarction, unplanned hospital admission for chest pain and cardiac death, occurred significantly less in the nicorandil than in the placebo group. Thus, preclinical and first clinical studies were 
conducted to evaluate a possible preconditioning effect of nicorandil against ischemia in nonhypertrophied hearts. However, to our knowledge, no data exist about nicorandil treatment of hypertrophied hearts.

The current study demonstrates that the administration of nicorandil during coronary occlusion preconditioned hypertrophied hearts to ischemia as indicated by the significant reduction of infarction size and preservation of EF. This reduction cannot be attributed to differences in the area at risk or heart rate, because there was no significant difference in these parameters between the groups.

In this acute infarction model, the preservation of myocardial viability that was due to drug treatment was not associated with an improvement in regional wall thickening at the site of the ischemic injury. Myocardial stunning may be responsible for the lack of improvement. Twenty-four hours after myocardial infarction, Lund et al. observed an improvement in wall thickening at the site of injury in nicorandil-treated nonhypertrophied rat hearts in comparison with untreated animals. ${ }^{27}$

Cardiac hypertrophy was induced in this study by aortic banding that resulted in a pressure overload. It has been shown that peripheral vascular resistance is higher in animals with aortic banding or coarctation than in the control. ${ }^{28}$ The natural course after banding is a progression to LV hypertrophy, dysfunction and eventually failure within weeks. ${ }^{29}$ The possible mechanism of the pronounced sensitivity of hypertrophied hearts towards ischemia might be the mismatch of number and size of capillaries and the size of myocytes. Capillary density is decreased by $20 \%$ in hypertrophied hearts compared with normal hearts, and the distance between each capillary is large, resulting in a lower oxygen concentration. . $^{30,31}$

MR imaging is considered an accurate method to determine normal and increased LV mass. ${ }^{9,11}$ Similar to findings in nonhypertrophied hearts, ${ }^{32}$ the LV mass of hypertrophied hearts determined with MR imaging correlated well with the postmortem weight of the LV in this study. Furthermore, MR imaging was a suitable tool to monitor the efficacy of new therapies designed to preserve myocardial function. The extent of Gadophrin-enhanced area correlated closely with that of postmortem histochemical staining. The specificity of Gadophrin in delineating necrotic tissue is explained by intracellular precipitation or binding to cellular debris. ${ }^{12}$ Thus, necrotic tissue that will not recover from the ischemic episode can be determined noninvasively with great precision by the use of Gadophrin-3 also in hypertrophied hearts. Gadophrin-3 is currently an experimental contrast agent not approved for the use in humans.

\section{Conclusion}

A brief episode of ischemia caused severe injury in hypertrophied hearts. The effect of ischemia was significantly attenuated after the administration of nicorandil. MR imaging, as a non-invasive diagnostic tool, revealed the changes in myocardial infarction size 
and function in treated and untreated hypertrophied hearts as well as in nonhypertrophied hearts. It shows the therapeutic value of potassium-channel openers in preserving viability in hypertrophied hearts. The demonstration of a "window of opportunity" for myocardial preconditioning to ischemia by an ATP-sensitive potassium-channel opener suggests new directions in investigating mechanisms underlying cardiac hypertrophy.

\section{References}

1. Verdecchia P, Carini G, Circo A, et al. Left ventricular mass and cardiovascular morbidity in essential hypertension: the MAVI study. J Am Coll Cardiol 38:1829-1835. 2001

2. Koyanagi S, Eastham CL, Harrison DG, et al. Increased size of myocardial infarction in dogs with chronic hypertension and left ventricular hypertrophy. Circ Res 50:55-62. 1982

3. Sink JD, Pellom GL, Currie WD, et al. Response of hypertrophied myocardium to ischemia: correlation with biochemical and physiological parameters. J Thorac Cardiovasc Surg 81:865-872. 1981

4. Ito H, Taniyama $\mathrm{Y}$, Iwakura $\mathrm{K}$, et al. Intravenous nicorandil can preserve microvascular integrity and myocardial viability in patients with reperfused anterior wall myocardial infarction. J Am Coll Cardiol 33:654660. 1999

5. Yasuda T, Hashimura K, Matsu-ura Y, et al. Nicorandil, a hybrid between nitrate and ATP-sensitive potassium channel opener, preconditions human heart to ischemia during percutaneous transluminal coronary angioplasty. Jpn Circ J 65:526-530. 2001

6. Effect of nicorandil on coronary events in patients with stable angina: the Impact Of Nicorandil in Angina (IONA) randomised trial. Lancet 359:1269-1275. 2002

7. Saeed M, Bremerich J, Wendland MF, et al. Reperfused myocardial infarction as seen with use of necrosis-specific versus standard extracellular MR contrast media in rats. Radiology 213:247-257. 1999

8. Pislaru SV, Ni Y, Pislaru C, et al. Noninvasive measurements of infarct size after thrombolysis with a necrosis-avid MRI contrast agent. Circulation 99:690-696. 1999

9. Bottini PB, Carr AA, Prisant LM, et al. Magnetic resonance imaging compared to echocardiography to assess left ventricular mass in the hypertensive patient. Am J Hypertens 8:221-228. 1995

10. Lorenz $\mathrm{CH}$, Walker ES, Morgan VL, et al. Normal human right and left ventricular mass, systolic function, and gender differences by cine magnetic resonance imaging. J Cardiovasc Magn Reson 1:7-21. 1999

11. Myerson SG, Montgomery HE, World MJ, et al. Left ventricular mass: reliability of M-mode and 2dimensional echocardiographic formulas. Hypertension 40:673-678. 2002

12. Ni Y, Petre C, Miao Y, et al. Magnetic resonance imaging-histomorphologic correlation studies on paramagnetic metalloporphyrins in rat models of necrosis. Invest Radio/ 32:770-779. 1997

13. Marchal G, Ni Y, Herijgers P, et al. Paramagnetic metalloporphyrins: infarct avid contrast agents for diagnosis of acute myocardial infarction by MRI. Eur Radiol 6:2-8. 1996

14. Gomma AH, Purcell HJ, Fox KM. Potassium channel openers in myocardial ischaemia: therapeutic potential of nicorandil. Drugs 61:1705-1710. 2001

15. Sanada S, Kitakaze M, Asanuma $H$, et al. Role of mitochondrial and sarcolemmal K(ATP) channels in ischemic preconditioning of the canine heart. Am J Physiol Heart Circ Physiol 280:H256-263. 2001

16. Holmuhamedov EL, Wang L, Terzic A. ATP-sensitive $\mathrm{K}+$ channel openers prevent Ca2+ overload in rat cardiac mitochondria. J Physiol 519:347-360. 1999

17. Haruna T, Horie M, Kouchi I, et al. Coordinate interaction between ATP-sensitive $\mathrm{K}+$ channel and $\mathrm{Na+}, \mathrm{K}+-$ ATPase modulates ischemic preconditioning. Circulation 98:2905-2910. 1998

18. Sakai K, Akima M, Saito K, et al. Nicorandil metabolism in rat myocardial mitochondria. J CardiovasC Pharmacol 35:723-728. 2000 
19. Lepran I, Koltai M, Siegmund W, et al. Coronary artery ligation, early arrhythmias, and determination of the ischemic area in conscious rats. J Pharmacol Methods 9:219-230. 1983

20. Patel DJ, Purcell HJ, Fox KM. Cardioprotection by opening of the K(ATP) channel in unstable angina. Is this a clinical manifestation of myocardial preconditioning? Results of a randomized study with nicorandil. CESAR 2 investigation. Clinical European studies in angina and revascularization. Eur Heart J 20:51-57. 1999

21. Yoneyama F, Satoh K, Taira N. Nicorandil increases coronary blood flow predominantly by K-channel opening mechanism. Cardiovasc Drugs Ther 4:1119-1126. 1990

22. Mizumura T, Nithipatikom K, Gross GJ. Infarct size-reducing effect of nicorandil is mediated by the KATP channel but not by its nitrate-like properties in dogs. Cardiovasc Res 32:274-285. 1996

23. Pieper GM, Gross GJ. Anti-free-radical and neutrophil-modulating properties of the nitrovasodilator, nicorandil. Cardiovasc Drugs Ther 6:225-232. 1992

24. Krombach GA, Higgins CB, Chujo $M$, et al. Blood pool contrast-enhanced MRI detects suppression of microvascular permeability in early postinfarction reperfusion after nicorandil therapy. Magn Reson Med 47:896-902. 2002

25. Das B, Sarkar C, Karanth KS. Effects of administration of nicorandil or bimakalim prior to and during ischemia or reperfusion on survival rate, ischemia/reperfusion-induced arrhythmias and infarct size in anesthetized rabbits. Naunyn Schmiedebergs Arch Pharmacol 364:383-396. 2001

26. Matsuo H, Watanabe S, Segawa T, et al. Evidence of pharmacologic preconditioning during PTCA by intravenous pretreatment with ATP-sensitive K+ channel opener nicorandil. Eur Heart J 24:1296-1303. 2003

27. Lund GK, Higgins CB, Wendland MF, et al. Assessment of nicorandil therapy in ischemic myocardial injury by using contrast-enhanced and functional MR imaging. Radiology 221:676-682. 2001

28. Stanek KA, Coleman TG, Murphy WR. Overall hemodynamic pattern in coarctation of the abdominal aorta in conscious rats. Hypertension 9:611-618. 1987

29. Norton GR, Woodiwiss AJ, Gaasch WH, et al. Heart failure in pressure overload hypertrophy. The relative roles of ventricular remodeling and myocardial dysfunction. J Am Coll Cardiol 39:664-671. 2002

30. Henquell L, Odoroff CL, Honig CR. Intercapillary distance and capillary reserve in hypertrophied rat hearts beating in situ. Circ Res 41:400-408. 1977

31. Anversa P, Beghi C, Kikkawa Y, et al. Myocardial infarction in rats. Infarct size, myocyte hypertrophy, and capillary growth. Circ Res 58:26-37. 1986

32. Manning WJ, Wei JY, Fossel ET, et al. Measurement of left ventricular mass in rats using electrocardiogram-gated magnetic resonance imaging. Am J Physiol 258:H1181-1186. 1990 


\section{Long-term oral treatment with nicorandil}

prevents the progression of left ventricular hypertrophy and preserves viability 


\section{Abstract}

Background: Left ventricular (LV) hypertrophy and myocardial infarction play important roles in the progressive LV dysfunction. We hypothesized that the potassium-channel opener and nitrate-like vasodilator nicorandil prevents the development of LV hypertrophy and preserves myocardial viability.

Methods: Twenty-four rats were subjected to aortic stenosis for 8 weeks to induce LV hypertrophy, and assigned to non-treated and nicorandil-treated (3 mg/kg/d) groups. A third group ( $\mathrm{n}=12$ ) without stenosis or treatment served as control. All 36 animals were subjected to reperfused infarction by 25-minute occlusion of the left coronary artery followed by 3 hours of reperfusion. Spin-echo magnetic resonance (MR) images were acquired to measure infarction size, LV mass, volumes, ejection fraction, and wall thickness. A necrosis-specific contrast agent, Gadophrin-3, was used to delineate necrotic myocardium. Aortic and LV pressures were measured invasively. At postmortem, LV mass and infarction size were determined and compared with MR findings.

Results: Nicorandil prevented the development of LV hypertrophy. Infarction size of nicorandil-treated animals was similar to control animals. Non-treated animals with aortic banding had higher LV mass $(P<0.001)$, lower ejection fraction $(P=0.006)$, and larger infarction size $(P<0.001)$ than treated and control animals. MR and postmortem data showed close agreement.

Conclusion: Nicorandil therapy prevented the development of cardiac hypertrophy and protected myocardium against ischemia. 


\section{Introduction}

Left ventricular (LV) hypertrophy following chronic pressure overload is an adaptive cardiac response at early stages, but is in the later course a major risk factor for cardiovascular morbidity and mortality. ${ }^{1}$ Regression of LV hypertrophy has been demonstrated in hypertensive patients treated with different classes of drugs. ${ }^{2,3}$ Nicorandil is a multi-action drug with selective potassium-channel opener and nitrate-like activity. ${ }^{4}$ To our knowledge, the long-term oral effect of nicorandil on the development of LV hypertrophy has not been previously investigated. The potassium channels have recently gained considerable interest in the context of myocardial ischemic preconditioning., ${ }^{5,6}$ Preconditioning of hypertrophied myocardium against ischemia has been demonstrated with administration of drugs such as angiotensin receptor blocker and insulin-like growth factor. ${ }^{7,8}$ Several clinical and experimental studies in non-hypertrophied myocardium have demonstrated a protection from ischemic injury by activation of the potassium channel. ${ }^{9-12}$

Magnetic resonance (MR) imaging has been employed with great precision in the non-invasive measurements of LV mass and function in normal and hypertrophied hearts. ${ }^{13,14}$ In addition, contrast-enhanced MR imaging with standard extracellular and necrosis-specific contrast agents has been used for detecting and sizing ischemic myocardial injury and is currently superior to other invasive and non-invasive imaging modalities. ${ }^{15,16}$ In this model of hypertrophy, a necrosis-specific MR contrast agent was used.

We hypothesized that chronic oral administration of nicorandil might prevent the development of LV hypertrophy and could result in a smaller infarction size compared with animals with myocardial hypertrophy subjected to myocardial ischemia. The specific aims of this study were as follows:

1. To determine the long-term oral effect of nicorandil therapy on the development of LV hypertrophy in rats subjected to aortic stenosis using MR imaging, hemodynamic measurements, and postmortem analysis.

2. To compare the LV geometry and infarct size in nicorandil-treated, hypertrophied and non-hypertrophied hearts, using the necrosis-specific contrast agent Gadophrin-3, hemodynamic assessment, and postmortem histochemical staining.

\section{Materials and methods}

\section{Animal model}

The experimental protocol was approved by the Committee on Animal Research at this institution and performed under the National Institutes of Health guidelines for the care and use of laboratory animals. Growing female Sprague Dawley rats ( $\mathrm{n}=36,90-110 \mathrm{~g}$ ) 
were assigned to 3 groups ( $n=12$ each); namely untreated with aortic stenosis, nicorandil-treated with aortic stenosis, and control (no aortic stenosis). Animals were anesthetized with a mixture of ketamine $(50 \mathrm{mg} / \mathrm{kg})$ and xylazine $(1.4 \mathrm{mg} / \mathrm{kg})$. The abdominal aorta was isolated and a steel wire of $0.4 \mathrm{~mm}$ diameter was placed close to the surface of suprarenal aorta. A thread was passed around both the aorta and the wire and tied to produce permanent aortic stenosis. Temporary closure of the abdominal aorta was confirmed by changing the color of the kidney from red to pale. Removal of the wire resulted in returning of the red color of the kidney. The abdominal muscle wall and skin was subsequently closed. Nicorandil treatment started 2 days after aortic stenosis and continued for 8 weeks. Nicorandil was dissolved in tap water and water consumption was measured biweekly to adjust the dose to $3 \mathrm{mg} / \mathrm{kg} / \mathrm{d}$.

Eight weeks later, all animals were re-anesthetized by intraperitoneal injection of sodium pentobarbital $(50 \mathrm{mg} / \mathrm{kg})$. Animals were mechanically ventilated with room air. After thoracotomy, the proximal portion of the first branch of left coronary artery was occluded for 25 minutes using a snare loop. Myocardial ischemia was documented by the changes in ST waves. During reperfusion 0.05 mmol/kg Gadophrin-3 was administered intravenously to delineate necrotic myocardium. The reperfusion period lasted $>3$ hours and was followed by MR imaging.

Necrosis-specific magnetic resonance contrast medium; Gadophrin-3

Gadophrin-3 (Schering AG, Berlin, Germany) is a porphyrin-based T1-enhancing gadolinium agent with $\mathrm{T} 1$ and $\mathrm{T} 2$ relaxivities of 8.9 and 12 seconds- $1 \mathrm{mM}-1$, respectively. ${ }^{17,18}$ During the reperfusion period the contrast agent accumulates in infarcted myocardium and clears from the plasma. Unlike extracellular agents, such as Gd-DTPA, Gadophrin-3 adheres to necrotic tissue or debris. ${ }^{19}$

\section{Magnetic resonance imaging}

Magnetic resonance imaging was performed with a 2.0 Tesla scanner system (Chemical Shift Imager, Bruker Instruments, Fremont, CA). Animals were placed supine in a birdcage imaging coil and connected to an ECG monitor to monitor heart rate and enable ECG-gating for image acquisition. Multislice T1-weighted spin echo images (TR/TE = $300 / 12 \mathrm{~ms} ;$ matrix $=256 \times 128$ data points; FOV $=50 \mathrm{~mm}$; slice thickness $=2 \mathrm{~mm}$, no gap between slices; acquisition time $=2.5 \mathrm{~min}$ ) were acquired to cover the entire heart in contiguous short axis views at end-diastolic (R-wave) and end-systolic phases (TR = $45 \%$ of $R-R$ interval). 
A polyethylene catheter was placed in the aorta via the right carotid artery and then in the LV to measure phasic and mean pressures with a rodent pressure transducer. Positive and negative maximum rate of LV pressure development (differential quotient of pressure change against time, $\mathrm{dP} / \mathrm{dt}$ ) was electronically derived from the pressure wave signal of LV. At the completion of the imaging protocol the left coronary artery was reoccluded with the same snare loop and $0.7 \mathrm{ml}$ of phthalocyanine blue dye injected into a tail vein. This dye imparted a blue color to normally perfused myocardium while the territory of the occluded artery (area at risk) remained unstained.

\section{Postmortem evaluation}

The heart was excised 3 minutes post-injection. The atria and right ventricle were removed and the LV weighed to determine LV mass. The LV was transversely sliced into 2$\mathrm{mm}$ slices to correspond to the location of MR images. The slices were incubated in $2 \%$ triphenyltetrazolium chloride solution (TTC) for 10 minutes at $38^{\circ} \mathrm{C}$. Viable myocardium appeared brick red while infarcted regions remained pale white. Upper and lower surfaces of the LV slices were digitally photographed to measure the area at risk and infarction size.

\section{Data analysis and statistics}

Magnetic resonance images and postmortem tissue slices were analyzed by a standard imaging software (NIH image version 1.59, National Institutes of Health, Bethesda, MD). By delineating LV boundaries (planimetry) on end-diastolic and end-systolic MR images and adding them together, LV volumes, mass, and ejection fraction were determined. Ejection fraction was calculated as: (end-diastolic volume - end-systolic volume)/enddiastolic volume. LV mass was calculated at end-diastole by subtracting the enddiastolic endocardial volume from the end-diastolic epicardial volume and then multiplying by the density of the myocardium $\left(1.05 \mathrm{~g} / \mathrm{cm}^{3}\right)$. The extent of the infarcted region as a percentage of the total LV was calculated as the sum of the Gadophrin-enhanced regions for all slices divided by the sum of the LV cross-sectional areas from all slices. The extent of the infarcted region determined with MR imaging as well as postmortem staining was also given as a percentage of the area at risk for infarction (infarction size in $g$ multiplied by 100 and divided by the area at risk in g). LV wall thickness (mm) was measured on end-systolic and end-diastolic images in a remote region (septal wall) and in the center of infarction on 2 slices per animal.

Percent systolic wall thickening was calculated as: [(systolic wall thickness - diastolic wall thickness)/diastolic wall thickness] $\times 100$. Stroke volume (SV) in $\mathrm{ml}$ was calculated from MR data as end-diastolic volume minus end-systolic volume. Cardiac output in 
$\mathrm{ml} / \mathrm{min}$ was calculated as SV multiplied by heart rate. Systemic vascular resistance in $\mathrm{mm} \mathrm{Hg} / \mathrm{l} / \mathrm{min}$ was calculated from MR data and pressure measurements as mean aortic pressure divided by cardiac output.

Data are expressed as mean values \pm SEM. To compare results between the three animal groups repeated-measures analysis of variance was employed to evaluate the significance of mean differences. If the $\mathrm{F}$ score from analysis of variance indicated a significant difference $(P<0.05)$, the comparison of individual mean differences was evaluated according to Bonferroni. Differences were considered significant if $P$ was $<$ 0.05 , per the number of individual comparisons. Bland-Altman analysis was used to determine the agreement between postmortem and MR measurements. Linear regression was also obtained to determine the correlation between MR imaging and postmortem measurements.

\section{Results}

Effect of oral nicorandil on the development of left ventricular hypertrophy

Magnetic resonance images of the entire hearts were obtained successfully in all 36 rats. Animals with aortic banding treated orally for 8 weeks with nicorandil showed an LV mass (MRI: $0.66 \pm 0.02$ g; postmortem: $0.66 \pm 0.02 \mathrm{~g}$ ) similar to control (0.62 \pm 0.02 g; $0.62 \pm 0.02 \mathrm{~g}$ ) as determined by MR imaging and at postmortem (Figure 1). In contrast, untreated animals with aortic banding had a significantly higher LV mass determined with MR imaging and at postmortem (0.81 $\pm 0.01 \mathrm{~g} ; 0.82 \pm 0.01 \mathrm{~g})$ compared with control and treated animals with aortic banding $(P<0.001)$. Bland-Altman analysis revealed a close agreement between the determination of LV mass in control $(0.001 \pm$ $0.005 \mathrm{~g})$, untreated $(-0.009 \pm 0.006 \mathrm{~g})$, and treated animals with aortic banding $(-0.026 \pm$ $0.004 \mathrm{~g}$ ) for the two methods, postmortem weighing and MR imaging (Figure 2). Regression analysis showed excellent correlation (treated animals: $r=0.98$; untreated: $r=$ 0.89; control: $r=0.98$ ). A comparison of the two methods is given in Figure 3. Nicorandil-treated animals had a tendency toward a higher body weight (treated: 278.0 \pm 5.6 g; untreated: $247.9 \pm 4.7$ g; control: $247.0 \pm 6.1$ g). However, this trend was not significant. 
MR Imaging

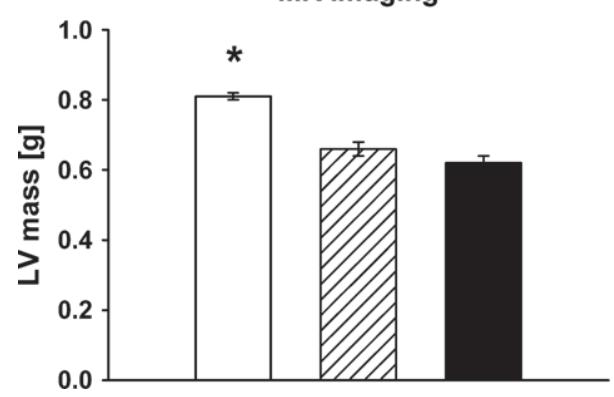

Postmortem

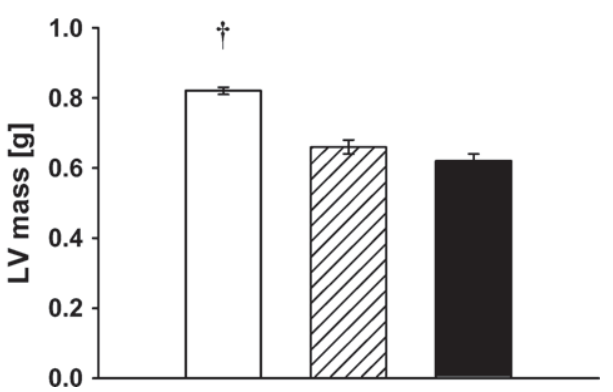

Figure 1: Bar chart showing mean values and standard error of left ventricular mass (LV mass) for non-treated (white) and long-term nicorandil-treated (cross-hatched box) animals with aortic banding as well as control animals (black box) measured with MR imaging and postmortem weighing. Long-term oral treatment with nicorandil prevented the development of left ventricular hypertrophy; ${ }^{*} p<0.001 ;+p<0.001$

\section{Effect of oral nicorandil on myocardial viability of hypertrophied hearts}

Three sets of MR images of cardiac short axis views representing the three animal groups are shown in Figure 4. All infarctions were located in the antero-lateral wall of the left ventricle. The figure shows the reperfused myocardial infarction as a bright region at 3 hours after the administration of the necrosis-specific contrast agent Gadophrin-3. It also demonstrates the difference in infarction size between the three groups. The infarction size was smaller in nicorandil-treated animals with aortic banding $(8.7 \pm$ $1.0 \%$ of LV) compared with untreated animals $(19.3 \pm 1.2 \%, P<0.001)$ and similar to control $(9.6 \pm 1.5 \%)$ on Gadophrin-enhanced MR images. Thus, nicorandil-treatment resulted in a reduction of infarction size compared with untreated animals with aortic banding. However, infarction size was not smaller than in control. A close agreement was found between postmortem and MR data regarding infarction size for control $(0.5$ $\pm 0.4 \%)$, untreated $(0.6 \pm 0.4 \%)$, and treated animals with aortic banding $(0.3 \pm 0.2 \%)$ by Bland-Altman analysis (Figure 2). Regression analysis indicated excellent correlation (treated animals: $r=0.98$; untreated: $r=0.95$; control: $r=0.97$ ). The area at risk for infarction (area supplied by the occluded artery) was the same in all three groups: $P=$ 0.7 for nicorandil-treated $(37.3 \pm 0.9 \%$ LV) and untreated animals with aortic banding $(38.3 \pm 1.3 \%)$ and control $(36.8 \pm 1.2 \%)$ (Figure 5$)$.

Expressed as \% of area at risk for infarction (\% of AaR), the infarction size determined with Gadophrin-enhanced MR imaging was $23.3 \pm 2.6 \%$ of AaR in nicorandiltreated animals with aortic banding, $50.4 \pm 3.2 \%$ of AaR $(P<0.001)$ in untreated animals, and $25.3 \pm 4.4 \%$ of AaR in control animals. A close agreement between postmortem and MR data was determined for the infarction size as \% of AaR for control $(0.7 \pm$ $0.6 \%)$, untreated $(0.7 \pm 1 \%)$, and treated animals with aortic banding $(0.9 \pm 0.7 \%)$ by Bland-Altman analysis. Regression analysis showed excellent correlation (treated animals: $r=0.96$; untreated: $r=0.96$; control: $r=0.99$ ). 
LVums: controt

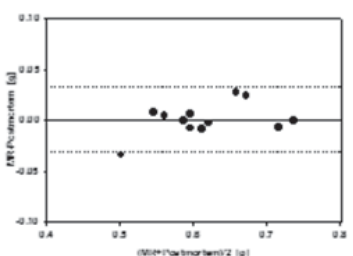

Intenton st: Controt

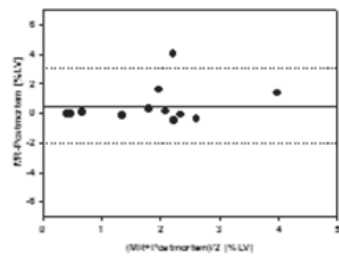

LV Mss: Hypottropty

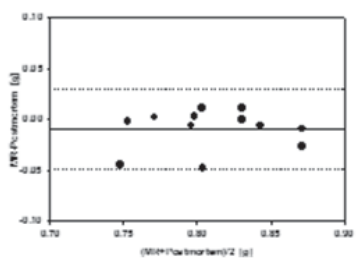

Introton Ul: wityportrophy

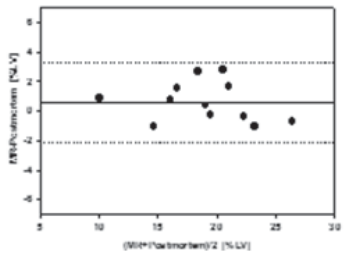

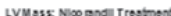

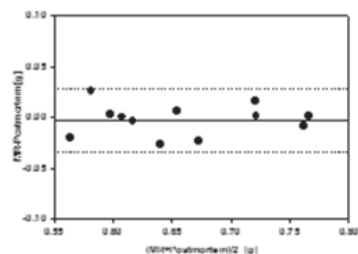

Intention szo: Nisoand IIT notmont

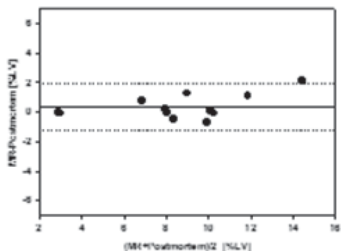

Figure 2: Bland-Altman plots.

Top row: Bland-Altman plots with standard deviation (2SD) of left ventricular mass (LV mass) determined with magnetic resonance imaging (MR) and postmortem histochemical staining (postmortem) for control animals without aortic banding (control, left), untreated animals with aortic banding (hypertrophy, middle) and longterm nicorandil-treated animals with aortic banding (nicorandil treatment, right).

Bottom row: Bland-Altman plots with standard deviation (2SD) of myocardial infarction size determined with magnetic resonance imaging (MR) and postmortem histochemical staining (postmortem) for control animals without aortic banding (control, left), untreated animals with aortic banding (hypertrophy, middle) and longterm nicorandil-treated animals with aortic banding (nicorandil treatment, right). [\% LV] denotes percentage of left ventricle in which contrast-enhancement or staining was observed.

LV Mass [g]

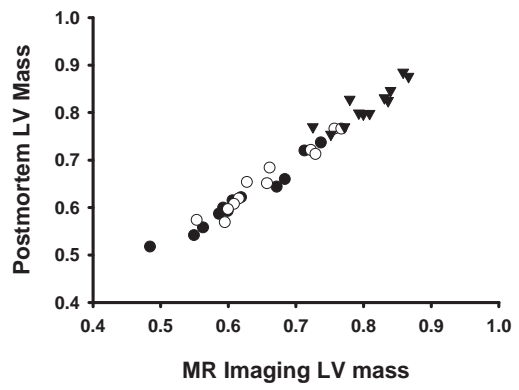

Infarction Size [g]

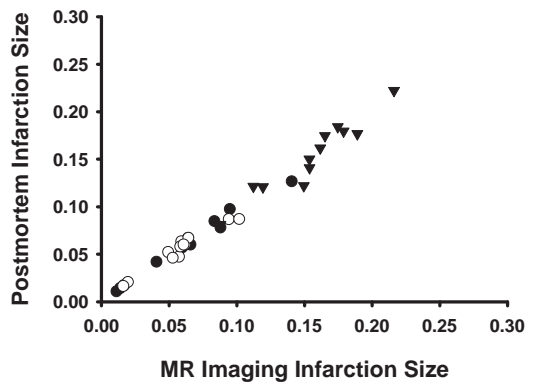

Figure 3: Comparison of MR imaging with postmortem analysis for the determination of left ventricular mass (left) and infarction size (right) for control animals without aortic banding (black dots, $n=12$ ), longterm nicorandil-treated animals with aortic banding (open dots, $n=12$ ) and untreated animals with aortic banding (triangles, $n=12$ ). A close agreement between the 2 methods was observed in all animal groups. 


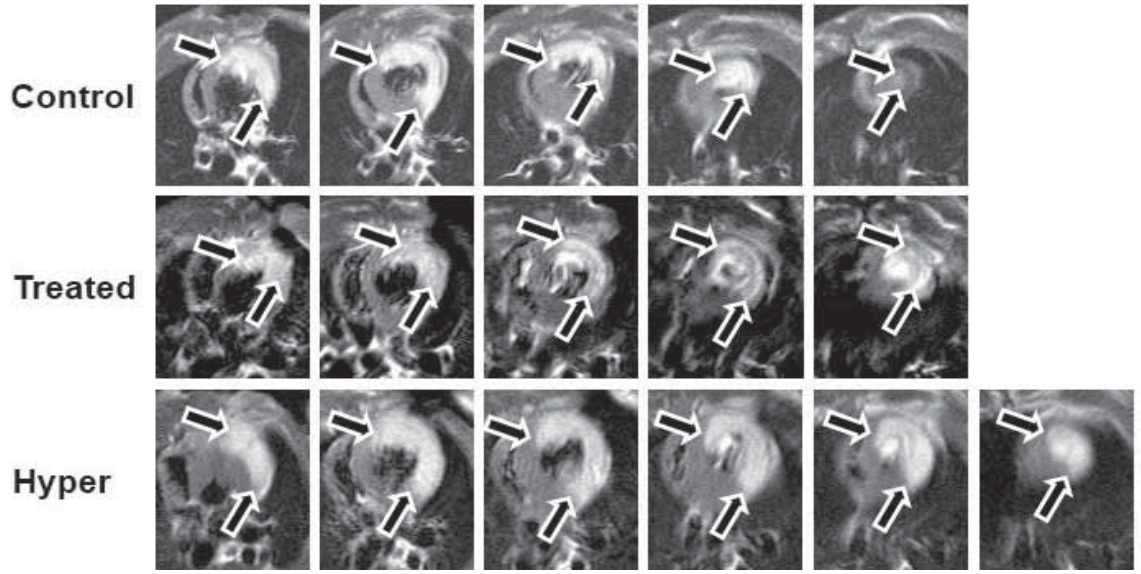

Figure 4: Short axis views of rat hearts subjected to $25 \mathrm{~min}$ of ischemia and $3 \mathrm{~h}$ of reperfusion, multislice Gadophrin-3-enhanced T1-weighted spin echo MR images. Top row: Control animal. Middle row: Longterm nicorandil-treated animal six weeks after aortic banding. Bottom row: Untreated animal with hypertrophied heart eight weeks after aortic banding. Infarction size is indicated by arrows. LV mass and infarction size of treated animals are comparable to control animals without aortic banding. The extend of the infarction is greater and more transmural in non-treated animals with cardiac hypertrophy in comparison with control and treated animals.

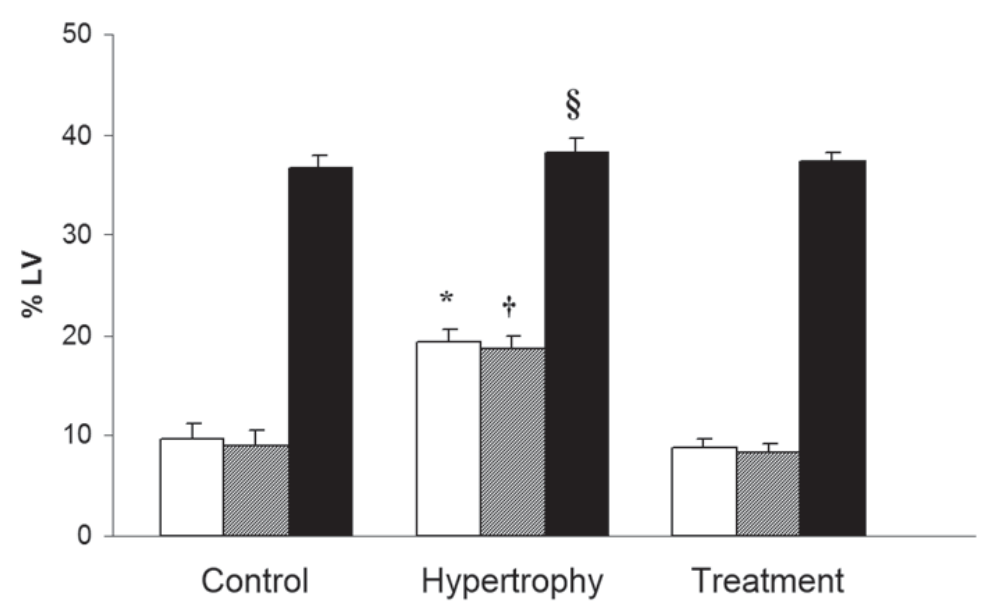

Figure 5: Bar graph showing infarction size as \% of left ventricular area in control, hypertrophied and oral treatment group determined with MR imaging (white boxes), postmortem TTC staining (grey) and area at risk determined with postmortem blue dye staining (black). The areas at risk are not different. Infarction size in treated and control animals is similar while infarction size in animals with hypertrophied hearts is much larger. * $p<0.001 ;+p<0.001 ; \S p=0.6$ 


\section{Effect of oral nicorandil on left ventricular function of hypertrophied hearts}

Hemodynamic parameters from MR imaging and invasive measurement data are given in Table 1. Heart rate, stroke volume, and cardiac output were not different between the three groups. The highest mean aortic blood pressure was measured in untreated animals with aortic banding while nicorandil treatment did not entirely lower the blood pressure to the value of control animals. The ejection fraction in nicorandil-treated animals with aortic banding was preserved and showed a value similar to control. The most severe impairment of ejection fraction was detected in untreated animals. This study showed that end-diastolic volume and LVEDP after infarction were significantly higher in hypertrophied hearts and both values were significantly decreased with the treatment of nicorandil. \%Wall thickening in infarcted regions was identical in all groups. \%Wall thickening in remote regions was identical in hypertrophied and control hearts while hypertrophied hearts showed less \%wall thickening. The wall thickness of untreated animals with aortic banding was higher than in control while treated animals had intermediate values.

Table 1: Parameters obtained from nicorandil-treated and untreated rats with aortic banding as well as control rats without aortic banding using MR imaging and catheterisation data.

\begin{tabular}{|c|c|c|c|c|}
\hline & Oral Treatment & Hypertrophy & Control & $P$ \\
\hline $\mathrm{HR}[/ \mathrm{min}]$ & $349 \pm 20$ & $354 \pm 12$ & $379 \pm 9$ & n.s. \\
\hline Mean BP [mmHg] & $94 \pm 3$ & $114 \pm 2$ & $81 \pm 2$ & $<0.001$ \\
\hline LVEDP [mmHg] & $5 \pm 1$ & $13 \pm 1$ & $5 \pm 1$ & 0.02 \\
\hline$+d P / d t$ & $8521 \pm 922$ & $4840 \pm 1126$ & $9416 \pm 900$ & 0.005 \\
\hline$-d P / d t$ & $7931 \pm 1101$ & $4240 \pm 994$ & $8833 \pm 1290$ & 0.01 \\
\hline $\mathrm{EDV}[\mathrm{ml}]$ & $0.166 \pm 0.010$ & $0.213 \pm 0.015$ & $0.164 \pm 0.006$ & 0.003 \\
\hline $\mathrm{EF}[\%]$ & $47.8 \pm 2.5$ & $38.0 \pm 3.1$ & $48.8 \pm 1.5$ & 0.006 \\
\hline $\mathrm{SV}[\mathrm{ml}]$ & $0.079 \pm 0.005$ & $0.080 \pm 0.007$ & $0.080 \pm 0.004$ & n.s. \\
\hline $\mathrm{CO}[\mathrm{ml} / \mathrm{min}]$ & $28.4 \pm 2.7$ & $28.5 \pm 2.6$ & $30.3 \pm 1.5$ & n.s. \\
\hline $\mathrm{SVR}[\mathrm{mmHg} / \mathrm{l} / \mathrm{min}]$ & $4286 \pm 1110$ & $4754 \pm 877$ & $2754 \pm 126$ & n.s. \\
\hline Wall thickness remote $[\mathrm{mm}]$ & $2.47 \pm 0.05$ & $2.89 \pm 0.10$ & $2.33 \pm 0.08$ & $<0.001$ \\
\hline Wall thickness infarct [mm] & $2.43 \pm 0.05$ & $2.93 \pm 0.10$ & $2.44 \pm 0.06$ & $<0.001$ \\
\hline \%WT remote [\%] & $38.1 \pm 0.85$ & $27.2 \pm 2.0$ & $35.0 \pm 1.6$ & $<0.001$ \\
\hline \%WT infarct [\%] & $4.1 \pm 0.6$ & $4.1 \pm 2.1$ & $3.7 \pm 0.7$ & n.s. \\
\hline
\end{tabular}

Mean \pm standard error for nicorandil-treated (oral treatment) and untreated animals (hypertrophy) with aortic banding and control (no aortic banding) ( $n=12$ animals per group). HR indicates heart rate; mean BP, mean aortic pressure. LVEDP, left ventricular end-diastolic pressure; +/- $\mathrm{dP} / \mathrm{dt}$, maximal rates of left ventricular pressure rise and decline; EDV, end-diastolic left ventricular volume; EF, ejection fraction; SV, stroke volume; CO, cardiac output; SVR, systemic vascular resistance; Wall thickness, diastolic wall thickness in remote and infarcted region; \%WT, percent systolic wall thickening; n.s., not significant. 


\section{Discussion}

Chronic treatment with nicorandil prevented the development of LV hypertrophy in rats subjected to aortic banding. It preserved myocardial viability and improved LV function. MR imaging is a non-invasive tool to monitor the effects of new therapies designed to protect myocardium from hypertrophy and infarction.

In this study, cardiac hypertrophy was induced by aortic banding resulting in pressure overload. The natural course after aortic banding is a progression to LV hypertrophy, dysfunction, and eventually failure. ${ }^{20}$ Treatment with nicorandil prevented the induction of LV hypertrophy and reduced infarction size by more than $50 \%$ compared with untreated animals with LV hypertrophy. Nicorandil is a clinically approved antianginal drug in Japan and several European countries with nitrate-like and adenosine triphosphate-sensitive potassium-channel ( $\mathrm{K}_{\text {ATP }}$ ) activating properties. ${ }^{21,22}$ Cardioprotective effects of nicorandil have been demonstrated in experimental and clinical studies by preconditioning myocardium to ischemia., ${ }^{53-26}$ Nicorandil acts on both vascular smooth muscles and cardiac myocytes. Activation of vascular $\mathrm{K}_{\text {ATP-channels leads to }}$ dilation of resistance arteries and capacitance veins, leading to reduction in pre- and after-load. Other studies have demonstrated that nicorandil increases coronary blood flow, reduces free radical activity, and prevents neutrophil adhesion. $5,23,27,28$

Nicorandil has not been used to investigate potential effects on the prevention of hypertrophy although previous work from our group indicated that chronic nicorandil therapy after myocardial infarction can influence the remodeling process by attenuating the increase of myocardial mass in non-infarcted areas. ${ }^{24}$ It has been shown that lowering the blood pressure with drugs such as amlodipine or vasodilators such as hydralazine did not attenuate the increase in heart weight in rats subjected to aortic banding. ${ }^{29}$ In contrast, nicorandil prevented the formation of LV hypertrophy in rats subjected to aortic stenosis, suggesting that the drug has other effects than merely vasodilation. Thus, this study could imply that the potassium channel might play an important role in the evolution of LV hypertrophy. Among others, two basic concepts could theoretically explain the cardioprotective and anti-hypertrophic effects of nicorandil in hearts prone to hypertrophy. Opening of the mitochondrial $\mathrm{K}_{\text {ATP-Channels in }}$ myocardium alters the cellular redox-state and prevents $\mathrm{Ca}^{2+}$ overload. It has also been shown that opening of the sarcolemmal $\mathrm{K}_{\text {ATP }}$-channels modulates cardiac $\mathrm{Na}^{+} / \mathrm{K}^{+}$-ATPase activity, shortens the action potential duration as well as reduces $\mathrm{Ca}^{2+}$ overload. ${ }^{30,31}$ Sanada et al. proposed a common pathway for induction of preconditioning, prevention of hypertrophy, and remodeling. This pathway involves opening of $\mathrm{K}_{\text {ATP-Channels }}$ and subsequent modulation of the 70-kDa S6 kinase. ${ }^{32}$ In addition, it is possible that the nitrate-like effect of nicorandil also plays a role in preventing myocardial hypertrophy. Nitrate vasodilators are thought to act by releasing nitric oxide, which is known to mediate vasodilation, inhibit platelet aggregation, and prevent leukocyte adhesion to endothelial cells. ${ }^{33}$ Chronic treatment with a nitric oxide synthesis inhibitor induces myocardial hypertro- 
phy, fibrosis and medial thickening and perivascular fibrosis of coronary arteries. ${ }^{34-36}$ However, the potassium-channel opener activity of nicorandil is also involved in the nitric oxide pathway. In the study mentioned above, Sanada et al. observed that opening of the adenosine triphosphate-sensitive potassium channel attenuated cardiac remodeling induced by long-term inhibition of nitric oxide synthesis. ${ }^{32}$ In addition, Horinaka et al. ${ }^{37}$ described that nicorandil enhanced cardiac endothelial nitric oxide synthase expression via the activation of KATP channel.

Similar to humans, coronary collateral circulation is minimal in the rat. A relatively short duration of coronary occlusion was chosen in this study to mimic a clinical situation in which a substantial volume of salvageable myocardium is present in the jeopardy zone. Infarction size in chronically nicorandil-treated animals with aortic banding was similar to control animals without aortic banding in the current study. The $\mathrm{K}_{\text {ATP }}$-channels located on the sarcolemma and the inner membrane of mitochondria of cardiomyocytes are important for the cardioprotection against ischemia and reperfusion injury. ${ }^{38-}$ ${ }^{40} \mathrm{O}^{\prime}$ Rourke ${ }^{41}$ found that nicorandil decreases cytoplasmic calcium levels by activating the ATP-dependent sarcolemmal calcium pump. Oral nicorandil therapy produced less reduction in infarction size compared with intravenous therapy in non-hypertrophied hearts subjected to infarction. ${ }^{42,43}$ This smaller reduction in infarction size may be related to the dose since a relatively low oral dose $(3 \mathrm{mg} / \mathrm{kg} / 24 \mathrm{~h})$ was used in the current study compared with an intravenous dose of $6.2 \mathrm{mg} / \mathrm{kg} / 3.5 \mathrm{~h} .{ }^{42,43}$ However, the prevention of cardiac hypertrophy might already explain the reduced infarction size compared with untreated animals with pressure overload. Thus, a future study is needed that includes long-term oral treatment of animals without aortic banding to study the influences of therapy on infarction size. In addition, different doses of nicorandil with different starting points of therapy in the course after aortic banding should be studied.

This study showed that LV end-diastolic volume and pressure after infarction were significantly higher in hypertrophied hearts and both values were significantly decreased with nicorandil treatment. It was also shown that $\pm \mathrm{dP} / \mathrm{dt}$ was significantly increased in treated compared with untreated animals, indicating that nicorandil improved LV function. Nicorandil therapy resulted in a lower blood pressure comparable to the blood pressure in control animals. However, since a single MR imaging and pressure measurement experiment was performed at the end of the 8-week period of aortic banding, the hemodynamic data are influenced by two parameters, namely myocardial infarction and presence or absence of pressure overload.

The underlying mechanism responsible for the prevention of LV hypertrophy, reduction of infarct size and preservation of LV function were not elucidated by this study. Nevertheless, the results of our descriptive study are the first to demonstrate an antihypertrophic effect of long-term therapy with nicorandil. Thus, further studies are needed to investigate these molecular mechanisms. The present results may help to encourage the introduction of new therapeutic strategies in myocardial hypertrophy. 
The present study demonstrated that MR imaging can serve as a precise and noninvasive tool to assess the efficacy of drug therapies. In a recent report, Bello et al. ${ }^{44}$ demonstrated the beneficial effect of beta-blockers in patients with heart failure using contrast-enhanced and functional MR imaging. Similar to results in non-hypertrophied

myocardium, ${ }^{45}$ we found in the current study that LV mass determined with MR imaging was nearly identical to that obtained at postmortem. In addition, infarction size determined with Gadophrin-enhanced MR imaging were similar to those obtained with histochemical staining in all animal groups. Thus, the accuracy and precision of a necrosisspecific MR contrast agent in sizing ischemic injury in hypertrophied hearts was demonstrated. Previous studies reported the potential of this class of MR contrast media in delineating acute infarction in non-hypertrophied hearts. ${ }^{18,42}$ Gadophrin-chelates specifically delineate acute, but not chronic, infarcts by intracellular precipitation or binding to cellular debris. ${ }^{46,47}$

\section{Conclusion}

Long-term oral treatment with nicorandil prevents the development of LV hypertrophy and preserves myocardial viability. While direct translation of this experimental model of cardiac hypertrophy to clinical conditions of hypertrophy might not be possible, these first results may encourage further studies to identify the molecular mechanisms of nicorandil in preventing myocardial hypertrophy and estimate the value of chronic nicorandil therapy in patients with myocardial hypertrophy.

\section{References}

1. Verdecchia P, Carini G, Circo A, et al. Left ventricular mass and cardiovascular morbidity in essential hypertension: the MAVI study. J Am Coll Cardiol. 2001;38:1829-1835.

2. Wachtell $\mathrm{K}$, Ibsen $\mathrm{H}$, Olsen $\mathrm{MH}$, et al. Albuminuria and cardiovascular risk in hypertensive patients with left ventricular hypertrophy: the LIFE study. Ann Intern Med. 2003;139:901-906.

3. Dahlof B, Pennert K, Hansson L. Reversal of left ventricular hypertrophy in hypertensive patients. A metaanalysis of 109 treatment studies. Am J Hypertens. 1992;5:95-110

4. Markham A, Plosker GL, Goa KL. Nicorandil. An updated review of its use in ischaemic heart disease with emphasis on its cardioprotective effects. Drugs. 2000;60:955-974

5. Effect of nicorandil on coronary events in patients with stable angina: the Impact Of Nicorandil in Angina (IONA) randomised trial. Lancet. 2002;359:1269-1275

6. Sakai K, Yamagata T, Teragawa $\mathrm{H}$, et al. Nicorandil enhances myocardial tolerance to ischemia without progressive collateral recruitment during coronary angioplasty. Circ J. 2002;66:317-322

7. Friehs I, Stamm C, Cao-Danh H, et al. Insulin-like growth factor-1 improves postischemic recovery in hypertrophied hearts. Ann Thorac Surg. 2001;72:1650-1656.

8. Butler KL, Huang AH, Gwathmey JK. AT1-receptor blockade enhances ischemic preconditioning in hypertrophied rat myocardium. Am J Physiol. 1999;277:H2482-H2487. 
9. Gross GJ, Auchampach JA. Blockade of ATP-sensitive potassium channels prevents myocardial preconditioning in dogs. Circ Res. 1992;70:223-233.

10. Garlid KD, Paucek P, Yarov-Yarovoy V, et al. Cardioprotective effect of diazoxide and its interaction with mitochondrial ATP-sensitive K+ channels. Possible mechanism of cardioprotection. Circ Res. 1997;81: 1072-1082.

11. Baines CP, Liu GS, Birincioglu M, et al. Ischemic preconditioning depends on interaction between mitochondrial KATP channels and actin cytoskeleton. Am J Physiol. 1999;276:H1361-H1368.

12. Sugimoto K, Ito H, Iwakura K, et al. Intravenous Nicorandil in Conjunction With Coronary Reperfusion Therapy is Associated With Better Clinical and Functional Outcomes in Patients With Acute Myocardial Infarction. Circ J. 2003;67:295-300.

13. Bottini PB, Carr AA, Prisant LM, et al. Magnetic resonance imaging compared to echocardiography to assess left ventricular mass in the hypertensive patient. Am J Hypertens. 1995;8:221-228

14. Myerson SG, Montgomery HE, World MJ, et al. Left ventricular mass: reliability of M-mode and 2dimensional echocardiographic formulas. Hypertension. 2002;40:673-678.

15. Kim RJ, Wu E, Rafael A, et al. The use of contrast-enhanced magnetic resonance imaging to identify reversible myocardial dysfunction. N Engl J Med. 2000;343:1445-1453.

16. Saeed M, Wendland MF, Bremerich GL, et al. Assessment of myocardial viability using standard extracellular and necrosis specific MR contrast media. Acad Radiol. 2002;9:S84-S87

17. Marchal G, Ni Y, Herijgers P, et al. Paramagnetic metalloporphyrins: infarct avid contrast agents for diagnosis of acute myocardial infarction by MRI. Eur Radiol. 1996;6:2-8

18. Pislaru SV, Ni Y, Pislaru C, et al. Noninvasive measurements of infarct size after thrombolysis with a necrosis-avid MRI contrast agent. Circulation. 1999;99:690-696

19. Hofmann B, Bogdanov A Jr, Marecos E, et al. Mechanism of gadophrin-2 accumulation in tumor necrosis. J Magn Reson Imaging. 1999;9:336-341.

20. Norton GR, Woodiwiss AJ, Gaasch WH, et al. Heart failure in pressure overload hypertrophy. The relative roles of ventricular remodeling and myocardial dysfunction. J Am Coll Cardiol. 2002;39:664-671.

21. Roland E. Safety profile of an anti-anginal agent with potassium channel opening activity: an overview. Eur Heart J. 1993;14:48-52.

22. Yasuda T, Hashimura K, Matsu-ura Y, et al. Nicorandil, a hybrid between nitrate and ATP-sensitive potassium channel opener, preconditions human heart to ischemia during percutaneous transluminal coronary angioplasty. Jpn Circ J. 2001;65:526-530

23. Mizumura T, Nithipatikom K, Gross GJ. Infarct size-reducing effect of nicorandil is mediated by the KATP channel but not by its nitrate-like properties in dogs. Cardiovasc Res. 1996;32:274-285.

24. Saeed $M$, Watzinger $N$, Krombach $G A$, et al. Left ventricular remodeling after infarction: sequential $M R$ imaging with oral nicorandil therapy in rat model. Radiology. 2002;224:830-837.

25. Sakai K, Yamagata T, Teragawa H, et al. Nicorandil-induced preconditioning as evidenced by troponin $T$ measurements after coronary angioplasty in patients with stable angina pectoris. Jpn Heart J. 2002;43:443-453.

26. Schalla S, Higgins CB, Chujo M, et al. Effect of potassium-channel opener therapy on reperfused infarction in hypertrophied hearts: demonstration of preconditioning by using functional and contrast-enhanced magnetic resonance imaging. J Cardiovasc Pharmacol Ther. 2004;9:193-202.

27. Yoneyama F, Satoh K, Taira N. Nicorandil increases coronary blood flow predominantly by K-channel opening mechanism. Cardiovasc Drugs Ther. 1990;4:1119-1126.

28. Pieper GM, Gross GJ. Anti-free-radical and neutrophil-modulating properties of the nitrovasodilator, nicorandil. Cardiovasc Drugs Ther. 1992;6:225-232.

29. Hiramatsu M, Furukawa T, Sawanobori T, et al. Ion channel remodeling in cardiac hypertrophy is prevented by blood pressure reduction without affecting heart weight increase in rats with abdominal aortic banding. J Cardiovasc Pharmacol. 2002;39:866-874.

30. Holmuhamedov EL, Wang L, Terzic A. ATP-sensitive K+ channel openers prevent Ca2+ overload in rat cardiac mitochondria. J Physiol. 1999;519:347-360. 
31. Haruna T, Horie M, Kouchi I, et al. Coordinate interaction between ATP-sensitive K+ channel and $\mathrm{Na+}, \mathrm{K}+-$ ATPase modulates ischemic preconditioning. Circulation. 1998;98:2905-2910.

32. Sanada S, Node K, Asanuma H, et al. Opening of the adenosine triphosphate-sensitive potassium channel attenuates cardiac remodeling induced by long-term inhibition of nitric oxide synthesis: role of 70-kDa S6 kinase and extracellular signal-regulated kinase. J Am Coll Cardiol. 2002;40:991-997.

33. Kelm M, Schrader J. Control of coronary vascular tone by nitric oxide. Circ Res. 1990;66:1561-1575.

34. Ribeiro MO, Antunes E, de Nucci G, et al. Chronic inhibition of nitric oxide synthesis. A new model of arterial hypertension. Hypertension. 1992;20:298-303.

35. Takemoto M, Egashira K, Usui $\mathrm{M}$, et al. Important role of tissue angiotensin-converting enzyme activity in the pathogenesis of coronary vascular and myocardial structural changes induced by long-term blockade of nitric oxide synthesis in rats. J Clin Invest. 1997;99:278-287.

36. Sanada S, Kitakaze M, Node K, et al. Differential subcellular actions of ACE inhibitors and AT(1) receptor antagonists on cardiac remodeling induced by chronic inhibition of NO synthesis in rats. Hypertension. 2001;38:404-411.

37. Horinaka S, Kobayashi N, Higashi T, et al. Nicorandil enhances cardiac endothelial nitric oxide synthase expression via activation of adenosine triphosphate-sensitive $\mathrm{K}$ channel in rat. J Cardiovasc Pharmacol. 2001;38:200-210

38. Terzic A, Jahangir A, Kurachi Y. Cardiac ATP-sensitive K+ channels: regulation by intracellular nucleotides and K+ channel-opening drugs. Am J Physiol. 1995;269:C525-C545.

39. Sato T, Sasaki N, O'Rourke B, et al. Nicorandil, a potent cardioprotective agent, acts by opening mitochondrial ATP-dependent potassium channels. J Am Coll Cardiol. 2000;35:514-518

40. Sanada S, Kitakaze M, Asanuma $H$, et al. Role of mitochondrial and sarcolemmal K(ATP) channels in ischemic preconditioning of the canine heart. Am J Physiol Heart Circ Physiol. 2001;280:H256-H263

41. O'Rourke ST. KATP channel activation mediates nicorandil-induced relaxation of nitrate-tolerant coronary arteries. J Cardiovasc Pharmacol. 1996;27:831-837.

42. Lund GK, Higgins CB, Wendland MF, et al. Assessment of nicorandil therapy in ischemic myocardial injury by using contrast-enhanced and functional MR imaging. Radiology. 2001;221:676-682.

43. Krombach GA, Higgins CB, Chujo $M$, et al. Blood pool contrast-enhanced MRI detects suppression of microvascular permeability in early postinfarction reperfusion after nicorandil therapy. Magn Reson Med. 2002;47:896-902.

44. Bello D, Shah DJ, Farah GM, et al. Gadolinium cardiovascular magnetic resonance predicts reversible myocardial dysfunction and remodeling in patients with heart failure undergoing beta-blocker therapy. Circulation. 2003;108:1945-1953

45. Manning WJ, Wei JY, Fossel ET, et al. Measurement of left ventricular mass in rats using electrocardiogram-gated magnetic resonance imaging. Am J Physiol. 1990;258:H1181-H1186

46. Ni Y, Petre C, Miao Y, et al. Magnetic resonance imaging-histomorphologic correlation studies on paramagnetic metalloporphyrins in rat models of necrosis. Invest Radiol. 1997;32:770-779

47. Watzinger N, Lund GK, Higgins CB, et al. The potential of contrast-enhanced magnetic resonance imaging for predicting left ventricular remodeling. J Magn Reson Imaging. 2002;16:633-640. 



\section{Incremental value of cardiovascular}

magnetic resonance over echocardiography in the detection of acute and chronic myocardial infarction

C. Jaarsma, S. Schalla, E.C. Cheriex, M.W. Smulders, I. van Dongen, P.J. Nelemans, A.P. Gorgels, J. Wildberger, H.J. Crijns, S.C. Bekkers.

J Cardiovasc Magn Reson. 2013 Jan 16;15:5. 


\section{Abstract}

Background: Although echocardiography is used as a first line imaging modality, its accuracy to detect acute and chronic myocardial infarction (MI) in relation to infarct characteristics as assessed with late gadolinium enhancement cardiovascular magnetic resonance (LGE-CMR) is not well described.

Methods: One-hundred-forty-one echocardiograms performed in 88 first acute STelevation MI (STEMI) patients, 2 (IQR1-4) days $(n=61)$ and 102 (IQR92-112) days post$\mathrm{MI}(\mathrm{n}=80)$, were pooled with echocardiograms of 36 healthy controls. 61 acute and 80 chronic echocardiograms were available for analysis (53 patients had both acute and chronic echocardiograms). Two experienced echocardiographers, blinded to clinical and CMR data, randomly evaluated all 177 echocardiograms for segmental wall motion abnormalities (SWMA). This was compared with LGE-CMR determined infarct characteristics, performed $104 \pm 11$ days post-MI. Enhancement on LGE-CMR matched the infarct-related artery territory in all patients (LAD 31\%, LCX 12\% and RCA 57\%).

Results: The sensitivity of echocardiography to detect acute MI was $78.7 \%$ and $61.3 \%$ for chronic Ml; specificity was $80.6 \%$. Undetected MI were smaller, less transmural, and less extensive (6\% [IQR3-12] vs. 15\% [IQR9-24], $50 \pm 14 \%$ vs. $61 \pm 15 \%, 7 \pm 3$ vs. $9 \pm 3$ segments, $p<0.001$ for all) and associated with higher left ventricular ejection fraction (LVEF) and non-anterior location as compared to detected MI (58 $\pm 5 \%$ vs. $46 \pm 7 \%, p<$ 0.001 and $82 \%$ vs. $63 \%, p=0.03)$. After multivariate analysis, LVEF and infarct size were the strongest independent predictors of detecting chronic $\mathrm{MI}(\mathrm{OR} 0.78[95 \% \mathrm{Cl} 0.68$ 0.88], $p<0.001$ and OR 1.22 [95\%Cl0.99-1.51], $p=0.06$, respectively). Increasing infarct transmurality was associated with increasing SWMA $(p<0.001)$.

Conclusions: In patients presenting with STEMI, and thus a high likelihood of SWMA, the sensitivity of echocardiography to detect SWMA was higher in the acute than the chronic phase. Undetected MI were smaller, less extensive and less transmural, and associated with non-anterior localization and higher LVEF. Further work is needed to assess the diagnostic accuracy in patients with non-STEMI. 


\section{Introduction}

Myocardial infarction (MI) is a major cause of death and disability worldwide. ${ }^{1}$ Accurate diagnosis is important, since it directs clinical management and affects prognosis. Despite the development of cardiac specific biomarkers (i.e. troponins) that increase several hours after the onset of myocardial ischemia, early diagnosis can still be difficult and $\mathrm{MI}$ may remain undetected. ${ }^{2}$ Non-invasive imaging modalities can improve the diagnosis of $\mathrm{MI}$, due to their ability to detect segmental wall motion abnormalities (SWMA) as a result of myocardial ischemia. ${ }^{3}$

Two-dimensional echocardiography has many advantages as a first line, bed-side, real-time imaging modality because it is inexpensive, and rapidly and widely available. Although echocardiography is subsidiary to the electrocardiogram (ECG) in hemodynamically stable patients presenting with ST-segment elevation myocardial infarction (STEMI), its role may be more important in patients with a non-diagnostic ECG. Echocardiography is generally agreed to be very accurate, but the sensitivity to detect acute MI varies widely. ${ }^{4}$ Sensitivities of up to $100 \%$ have been reported in small studies that investigated patients with previous $\mathrm{Ml}, \mathrm{Q}$-wave $\mathrm{Ml}$ and included patients with good image quality. ${ }^{5-8}$ However, the sensitivity ranged between $60-70 \%$ when predominantly non-Q-wave $\mathrm{Ml}$ and patients with less optimal image quality echocardiograms were included. ${ }^{9,10}$ Most studies have focused on the echocardiographic detection of acute MI, and less is known about its usefulness in chronic MI. ${ }^{11,12}$ Chronic MI may remain undetected more frequently, because of the disappearance of SWMA after several weeks. ${ }^{13}$

Animal studies have shown that the extent of echocardiographic SWMA is related to infarct size and transmurality. ${ }^{14}$ This is also suggested by early clinical studies, but less accurate measures of infarct size were used, such as peak enzyme release and the presence or absence of Q-waves. ${ }^{7,9,10}$ The purpose of this study was to investigate the diagnostic accuracy of echocardiography to detect SWMA in the acute and chronic phase in a well described homogeneous population initially presenting with STEMI and consequently a high likelihood of SWMA. Additionally, we investigated the relation of SWMA with underlying infarct characteristics as assessed with late gadolinium enhancement cardiovascular magnetic resonance (LGE-CMR).

\section{Methods}

\section{Study population}

The current study is a retrospective subanalysis of patients admitted with a first STelevation MI (STEMI). Patients were consecutively and prospectively enrolled between August 2006 and March 2008. The purpose of the main study was to investigate infarct characteristics at baseline and follow-up using LGE-CMR. ${ }^{15}$ For this subanalysis, only 
patients were studied who had echocardiograms during admission (acute), follow-up (chronic), or both available and in whom LGE-CMR was performed. All patients were referred for urgent coronary angiography and primary percutaneous coronary intervention (PCI) of the infarct related artery (IRA). The definition of STEMI was based on a consensus document that includes an appropriate rise and fall in cardiac biomarkers and electrocardiographic (ECG) changes indicative of new ischemia. ${ }^{16}$ Patients $\leq 18$ years, with regular contraindications for $\mathrm{CMR}$, and those with left bundle branch block were excluded. To represent daily clinical practice, patients were not excluded because of poor echocardiographic image quality. In total, 88 patients with 141 echocardiograms were studied (27 acute and 8 chronic echocardiograms were not available). In 53 patients, both acute and chronic echocardiograms were available. During the study period, an arbitrary number of 36 consecutive subjects were extracted from a hospital database to serve as a control group and determine specificity. These subjects were either healthy volunteers or analyzed for various reasons. All were finally diagnosed without cardiac disease and none had evidence of myocardial scar (on LGE-CMR) or significant coronary artery disease (normal invasive coronary angiography, coronary computed tomography, or exercise treadmill test). Maastricht University Medical Center is a high volume center for cardiac ultrasound (13.000 transthoracic and 1000 transoesophageal examinations per year). Informed consent was obtained in all patients and Review Board of Maastricht University Medical Center approved the study.

\section{Echocardiography}

Transthoracic echocardiography was performed at a median of 2 (interquartile range [IQR] 1-4) days (acute) and 102 (IQR 92-112) days (chronic) after admission. Echocardiography was performed according to the American Society of Echocardiography guidelines using a commercially available ultrasound system (Sonos 5500 systems with S3 transducers or iE33 systems with S5-1 transducers, Philips Medical Systems, Best, The Netherlands). ${ }^{17}$ All images were acquired in supine or left lateral decubitus position and recorded as ECG-gated digital loops and stored for off-line analysis. Standard parasternal short and long axis views and apical two-, four-, and five-chamber views were obtained. No myocardial contrast enhanced technique was used.

All 177 echocardiographic studies (patients and controls) were analyzed independently and in random order by two board-certified cardiologists (S.S. and S.B.) with $>10$ years of experience in echocardiography, who were blinded to patient, clinical and CMR data. Discrepancies were resolved in consensus after review by a third expert (E.C.). Left ventricular ejection fraction (LVEF) was estimated visually. Regional wall motion was assessed visually according to the AHA 17-segment model on a four-point scale $\left(0=\right.$ normal, $1=$ hypokinesia, $2=$ akinesia, and $3=$ dyskinesia) ${ }^{18}$ Since segments could be visualized in more than one view, a final consensus score was assigned by combining all views. Finally, the presence or absence of MI was assessed (defined as SWMA-score 
$\geq 1$ in $\geq 1$ segment, with or without wall thinning). Image quality was scored on a threepoint scale based on the number of interpretable segments (i.e. $0=$ poor, if $>1$ segment was not interpretable in any view; $1=$ average, if all segments were interpretable but not in all views; and $2=e x c e l l e n t$, if all views were interpretable in all views). MI localization was classified as anterior (left anterior descending [LAD] artery territory) or nonanterior (left circumflex [LCX] or right coronary artery [RCA] territory). Only when the localization of SWMA on echocardiography and infarct on LGE-CMR matched, MI was defined as being correctly detected by echocardiography.

\section{Cardiovascular magnetic resonance}

Patients underwent CMR at a mean of $5 \pm 2$ days (acute) and $104 \pm 11$ days (chronic) after admission. CMR was performed for research purposes (i.e. not clinically ordered scans), and scan results were not used to guide clinical decision-making. Images were acquired with a 1.5 Tesla system (Philips Intera, Philips Medical Systems, Best, The Netherlands) equipped with a cardiac software package and five-element phased array surface coil. Although our CMR protocol included cine (steady-state free precession) and T2weighted (black-blood turbo spin echo with fat suppression) imaging, only the LGE-CMR images were used for the purpose of this study. LGE-CMR images were acquired 10 minutes after administration of $0.2 \mathrm{mmol} / \mathrm{kg}$ body weight Gadolinium diethylenetriaminepentaacetic acid (Gd-DTPA, Magnevist ${ }^{\circledR}$, Bayer-Schering, Germany), using a breathhold three-dimensional inversion-recovery gradient echo technique (acquired/reconstructed slice thickness $12 / 6 \mathrm{~mm}$, average TR/TE 3.9/2.4ms, multi-shot [50 profiles/shot] segmented partial echo readout, flip angle $15^{\circ}$, FOV $400 \mathrm{~mm}$, matrix 256x256) in the short axis, two-chamber and four-chamber views. ${ }^{15}$ Inversion delay time was set to null signal from normal myocardium (typically 200-280ms).

CMR images were analyzed independently by two observers, experienced in reading CMR and blinded to clinical and echocardiographic data, using commercially available software (CAAS MRV 3.0, Pie Medical Imaging, Maastricht, The Netherlands). The endocardial and epicardial borders were manually traced on the LGE-CMR short axis images, excluding the papillary muscles, to determine infarct size and transmurality. Infarct size and transmurality were measured by manually tracing enhanced areas (including areas of microvascular obstruction) and expressed as a percentage of LV mass and segmental LV wall thickness, respectively. CMR and echocardiographic images were analyzed on separate occasions.

\section{Statistical analysis}

Continuous variables with normally distributed data are expressed as mean \pm standard deviation (SD), otherwise as median with IQR. Categorical data are expressed as frequencies with percentages. The inter- and intraobserver agreement between the two 
readers was analyzed by using Cohen's kappa (к) coefficient. Differences in categorical data were evaluated using a Chi-square or Fisher's exact test. For continuous data, the independent $t$-test was used for normally distributed data and the Mann-Whitney $U$ test when not normally distributed.

Validity of echocardiography for the diagnosis of acute and chronic MI was evaluated by calculation of sensitivity and specificity with corresponding 95\% confidence intervals $(\mathrm{CI})$. LGE-CMR was used as reference standard. Differences in sensitivity between acute and chronic MI were tested using a test for paired proportions.

Univariate binary logistic regression was performed to explore the effect of different infarct characteristics on accurate detection of Ml by echocardiography. The dependent variable in this analysis was whether or not MI was detected by echocardiography (detected versus undetected). Independent variables associated with a $p$-value $<0.05$ were selected for inclusion in a multivariate binary logistic regression model in order to evaluate the independent effects of specific infarct characteristics. One variable per every 7-10 events were considered acceptable to be included into the multivariate model, where an event is defined as the outcome that is the least frequent. ${ }^{19}$ SPSS version 17.0 (SPSS Inc., Chicago, Illinois) was used for all statistical analyses. A two-tailed value of $p<0.05$ was considered statistically significant.

\section{Results}

\section{Baseline characteristics}

Patients with $\mathrm{MI}$ were older and more often active or ex-smokers than healthy controls ( $59 \pm 11$ vs. $43 \pm 12$ years and $86 \%$ vs. $11 \%$, respectively, $p<0.001$ for both, Table 1 ). In patients, post-PCI thrombolysis in myocardial infarction (TIMI 3) flow was established in 88\%. The infarct-related artery (IRA) was the LAD in 31\%, LCX in $12 \%$ and RCA in 57\% of patients, and approximately half had single vessel disease (51\%). In all patients, enhancement was visible on LGE-CMR images matching the territory of the IRA. Infarct size and

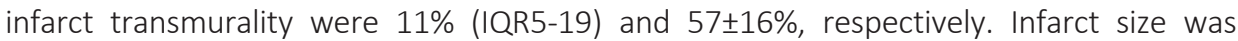
smaller and LVEF higher in patients with non-anterior $\mathrm{MI}$ than in patients with anterior $\mathrm{MI}$ (10\% [IQR5-15] vs. 23\% [IQR13-28] and 52 $\pm 8 \%$ vs. $45 \pm 10 \%$, respectively, $p<0.001$ for both).

\section{Intraobserver and interobserver agreement}

Analysis of intraobserver variability of echocardiographic assessment showed an agreement of $80 \%(\mathrm{k}=0.58)$ and $85 \%(\mathrm{k}=0.63)$ for observer 1 and 2, respectively. Analysis of the interobserver variability showed an agreement of $85 \%(k=0.70)$. The interobserver agreement for measuring infarct size on LGE-CMR images was excellent ( $k=0.90)$. 
Table 1: Baseline characteristics

\begin{tabular}{|c|c|c|c|}
\hline & $\begin{array}{l}\text { Patients }(n=88) \\
n=\end{array}$ & Controls $(n=36)$ & $\mathrm{p}$-value \\
\hline Age (y) & $59 \pm 11$ & $43 \pm 12$ & $<0.001$ \\
\hline Male (\%) & $65(74)$ & $20(56)$ & 0.06 \\
\hline Diabetes mellitus (\%) & $6(7)$ & $1(3)$ & 0.68 \\
\hline Smoking (\%) & $76(86)$ & $4(11)$ & $<0.001$ \\
\hline Hypertension (\%) & $34(39)$ & 7 (19) & 0.80 \\
\hline Hypercholesterolemia (\%) & $25(28)$ & $4(11)$ & 0.18 \\
\hline Positive family history (\%) & $41(47)$ & $14(39)$ & 0.84 \\
\hline \multicolumn{4}{|l|}{ Coronary Angiography } \\
\hline \multicolumn{4}{|l|}{ Infarct related artery (\%) } \\
\hline LAD & $27(31)$ & - & \\
\hline $\mathrm{LCx}$ & $11(12)$ & - & \\
\hline RCA & $50(57)$ & - & \\
\hline \multicolumn{4}{|c|}{ Number of diseased vessels (\%) } \\
\hline 1 & $45(51)$ & - & \\
\hline$\geq 2$ & $43(49)$ & - & \\
\hline \multicolumn{4}{|l|}{ TIMI 3 (\%) } \\
\hline Pre-PCl & $8(9)$ & - & \\
\hline Post-PCl & $77(88)$ & - & \\
\hline
\end{tabular}

\section{Echocardiography}

Days post MI

$\begin{array}{ll}\text { Acute } & 2(1-4) \\ \text { Chronic } & 102(92-112)\end{array}$

Image quality (\%)

$\begin{array}{lll}\text { Excellent } & 42(30) & 9(25) \\ \text { Average } & 87(62) & 24(67) \\ \text { Poor } & 12(8) & 3(8)\end{array}$

CMR

Days post MI

Acute

$5 \pm 2$

Chronic

$\begin{array}{lll}\text { Chronic } & 104 \pm 11 & \\ \text { Days between chronic echo and CMR } & 0(0-4) & 38 \text { (13-76) } \\ \text { Infarct size, \% of LV } & 11(5-19) & - \\ \text { Infarct transmurality, \% } & 57 \pm 16 & - \\ \text { Number of infarcted segments } & 8 \pm 3 & -\end{array}$

Values are presented as mean \pm standard deviation, median and interquartile range or proportions (\%) when appropriate. $L A D=$ left anterior descending artery; $L C x=$ left circumflex artery; RCA = right coronary artery; $\mathrm{TIMI}=$ thrombolysis in myocardial infarction $\mathrm{PCI}=$ percutaneous coronary intervention; $\mathrm{MI}=$ myocardial infarction; $C M R$ = cardiovascular magnetic resonance; $L V E F=$ left ventricular ejection fraction. 
The diagnostic performance of echocardiography to detect $\mathrm{Ml}$ is shown in Table 2. Overall, MI was detected by echocardiography in 97 out of 141 studies, resulting in an overall sensitivity of $68.8 \%$. Forty-eight out of the 61 patients with acute $\mathrm{MI}$, and 49 out of the 80 patients with chronic MI were detected, resulting in a sensitivity of $78.7 \%$ and $61.3 \%$, respectively. In the 53 patients in whom both acute and chronic echocardiograms were available for analysis, sensitivity for acute and chronic MI were $75.4 \%$ (40/53) and $67.9 \%$ (36/53). This was not significantly different as compared to the total group ( $p=0.84$ and $p=0.06$, respectively). The sensitivity to detect LCX-related $\mathrm{MI}$ was somewhat higher than RCA-related $\mathrm{MI}$, but this did not reach statistical significance (70.0\% vs. $61.0 \%$, respectively, $p=0.46$ )

Overall, the localization of observed SWMA did not match the localization of infarction on LGE-CMR in 4 out of 141 studies (2.8\%) and more frequently so in chronic than in acute $\mathrm{MI}(3.8 \%$ vs. $1.6 \%)$. In healthy controls, 7 out of 36 studies were incorrectly classified as $\mathrm{Ml}$, resulting in a specificity of $80.6 \%$. All false positive assessments concerned the basal inferior or basal inferolateral segments.

Table 2: Diagnostic performance of echocardiography to detect myocardial infarction

\begin{tabular}{llllll}
\hline & $\mathrm{N}$ & $\begin{array}{l}\text { Sensitivity } \\
95 \% \mathrm{Cl}\end{array}$ & $\begin{array}{l}\text { Specificity } \\
95 \% \mathrm{Cl}\end{array}$ & $\begin{array}{l}\mathrm{PPV} \\
95 \% \mathrm{Cl}\end{array}$ & $\begin{array}{l}\mathrm{NPV} \\
95 \% \mathrm{Cl}\end{array}$ \\
\hline Overall & \multirow{2}{*}{141} & $68.8 \%$ & $80.6 \%$ & $93.3 \%$ & $39.7 \%$ \\
& & $(64.9-71.4 \%)$ & $(65.3-90.9 \%)$ & $(88.0-96.8 \%)$ & $(32.2-44.8 \%)$ \\
Acute MI & \multirow{2}{*}{61} & $78.7 \%$ & $80.6 \%$ & $87.3 \%$ & $69.0 \%$ \\
& & $(70.8-84.4 \%)$ & $(67.1-90.2 \%)$ & $(78.5-93.6 \%)$ & $(57.6-77.3 \%)$ \\
Chronic MI & \multirow{2}{*}{80} & $61.3 \%$ & $80.6 \%$ & $87.5 \%$ & $48.3 \%$ \\
& & $(54.8-65.8 \%)$ & $(66.1-90.7 \%)$ & $(78.2-94.0 \%)$ & $(39.7-54.4 \%)$ \\
\hline
\end{tabular}

$\mathrm{Cl}=$ confidence interval; $\mathrm{PPV}=$ positive predictive value; $\mathrm{NPV}=$ negative predictive value; $\mathrm{MI}=$ myocardial infarction.

\section{Characteristics of detected and undetected myocardial infarction}

Echocardiographically undetected $\mathrm{MI}$ were more often non-anteriorly located in comparison to detected MI ( $82 \%$ vs. $63 \%, p=0.03$, Table 3). The prevalence of multivessel disease did not differ between patients with undetected and detected $\mathrm{Ml}(p=0.85)$. Overall, undetected MI were smaller, less transmural, involved less segments, and were associated with higher LVEF as compared to detected MI (6\% [IQR3-12] vs. 15\% [IQR924], $50 \pm 14 \%$ vs. $61 \pm 15 \%, 7 \pm 3$ vs. $9 \pm 3$ segments, and $58 \pm 5 \%$ vs. $46 \pm 7$, respectively, $p<0.001$ for all). Image quality was not different between patients with undetected and detected MI. Similar associations with infarct characteristics were found in the 53 patients who had both acute and chronic echocardiograms. 
Table 3: Comparison of characteristics between detected and undetected myocardial infarctions

\begin{tabular}{|c|c|c|c|}
\hline & $\begin{array}{l}\text { Detected } \\
(n=97)\end{array}$ & $\begin{array}{l}\text { Undetected } \\
(n=44)\end{array}$ & $p$-value \\
\hline Age (y) & $60 \pm 12$ & $59 \pm 10$ & 0.81 \\
\hline Male (\%) & $74(76)$ & $33(75)$ & 0.87 \\
\hline Infarct localization (\%) & & & $<0.05$ \\
\hline Anterior & $36(37)$ & $8(18)$ & \\
\hline Non-anterior & $61(63)$ & $36(82)$ & \\
\hline Number of diseased vessels & & & 0.85 \\
\hline 1 & $49(51)$ & $23(52)$ & \\
\hline$\geq 2$ & $48(49)$ & $21(48)$ & \\
\hline \multicolumn{4}{|l|}{ Echocardiography } \\
\hline \multicolumn{4}{|l|}{$\operatorname{LVEF}(\%)$} \\
\hline Acute & $44 \pm 7$ & $58 \pm 7$ & $<0.001$ \\
\hline Chronic & $48 \pm 7$ & $59 \pm 5$ & $<0.001$ \\
\hline \multicolumn{4}{|l|}{ Days post MI } \\
\hline Acute & $2.0(1.0-3.0)$ & $4.0(3.0-6.5)$ & 0.001 \\
\hline Chronic & $101(91-113)$ & $104(97-111)$ & 0.50 \\
\hline Image quality (\%) & & & 0.37 \\
\hline Excellent & $26(27)$ & $16(36)$ & \\
\hline Average & $61(63)$ & $26(59)$ & \\
\hline Poor & $10(10)$ & $2(5)$ & \\
\hline \multicolumn{4}{|l|}{ CMR } \\
\hline \multicolumn{4}{|l|}{ Infarct size, $\%$ of LV } \\
\hline Acute & $16(10-25)$ & $6(3-13)$ & 0.002 \\
\hline Chronic & $15(8-23)$ & $6(3-11)$ & $<0.001$ \\
\hline \multicolumn{4}{|l|}{ Infarct transmurality, \% } \\
\hline Acute & $62 \pm 14$ & $54 \pm 17$ & 0.07 \\
\hline Chronic & $61 \pm 16$ & $48 \pm 13$ & $<0.001$ \\
\hline \multicolumn{4}{|l|}{ Number of infarcted segments } \\
\hline Acute & $9 \pm 3$ & $7 \pm 3$ & 0.02 \\
\hline Chronic & $9 \pm 3$ & $7 \pm 3$ & 0.005 \\
\hline
\end{tabular}

Values are presented as mean \pm standard deviation, median and interquartile range or proportions (\%) when appropriate; $\mathrm{MI}=$ myocardial infarction; $\mathrm{CMR}=$ cardiovascular magnetic resonance; $\mathrm{LVEF}=$ left ventricular ejection fraction.

\section{Acute versus chronic myocardial infarction}

In both acute and chronic MI, LVEF was significantly higher in undetected than in detected $\mathrm{MI}(58 \pm 7 \%$ vs. $44 \pm 7 \%$ and $59 \pm 5 \%$ vs. $48 \pm 7 \%$, respectively, $p<0.001$ for both, 
Table 3) and the probability of detecting $\mathrm{MI}$ increased as LVEF decreased ( $b=-0.30$ and $b=-0.27$, respectively, $p<0.001$ for both, Table 4).

Table 4: Univariate and multivariate logistic regression analysis for the prediction of the echocardiographic detection of myocardial infarction

\begin{tabular}{|c|c|c|c|c|c|c|}
\hline & \multicolumn{3}{|l|}{ Univariate } & \multicolumn{3}{|l|}{ Multivariate } \\
\hline & $\begin{array}{c}\text { Coefficient } \\
\text { (B) }\end{array}$ & $\begin{array}{c}\text { OR } \\
(95 \% \mathrm{Cl})\end{array}$ & p-value & $\begin{array}{c}\text { Coefficient } \\
\text { (B) }\end{array}$ & $\begin{array}{c}\text { OR } \\
(95 \% \mathrm{Cl})\end{array}$ & p-value \\
\hline \multicolumn{7}{|l|}{ Acute MI } \\
\hline \multicolumn{7}{|l|}{ Infarct localization } \\
\hline Anterior vs. non-anterior & 2.06 & $7.86(0.94-65.5)$ & 0.06 & & & \\
\hline \multicolumn{7}{|l|}{ Image quality } \\
\hline Poor vs. excellent & 0.49 & $1.64(0.14-19.4)$ & 0.70 & & & \\
\hline Average vs. excellent & -0.06 & $0.94(0.22-4.11)$ & 0.93 & & & \\
\hline Time point echocardiography & -0.32 & $0.72(0.56-0.94)$ & 0.014 & & & \\
\hline LVEF & -0.30 & $0.74(0.63-0.87)$ & $<0.001$ & & & \\
\hline Infarct size & 0.16 & $1.17(1.05-1.31)$ & 0.006 & & & \\
\hline Infarct transmurality & 0.04 & $1.04(1.00-1.09)$ & 0.08 & & & \\
\hline Number of infarcted segments & 0.28 & $1.33(1.04-1.71)$ & 0.026 & & & \\
\hline \multicolumn{7}{|l|}{ Chronic MI } \\
\hline \multicolumn{7}{|l|}{ Infarct localization } \\
\hline Anterior vs. non-anterior & 0.60 & $1.82(0.65-5.09)$ & 0.25 & & & \\
\hline \multicolumn{7}{|l|}{ Image quality } \\
\hline Poor vs. excellent & 1.24 & $3.47(0.34-35.1)$ & 0.29 & & & \\
\hline Average vs. excellent & 0.43 & $1.53(0.59-3.96)$ & 0.38 & & & \\
\hline Time point echocardiography & -0.02 & $0.98(0.94-1.01)$ & 0.18 & & & \\
\hline LVEF & -0.27 & $0.76(0.68-0.86)$ & $<0.001$ & -0.25 & $0.78(0.68-0.88)$ & $<0.001$ \\
\hline Infarct size & 0.16 & $1.17(1.08-1.28)$ & $<0.001$ & 0.20 & $1.22(0.99-1.51)$ & 0.06 \\
\hline Infarct transmurality & 0.06 & $1.06(1.02-1.10)$ & 0.001 & -0.02 & $0.98(0.92-1.04)$ & 0.46 \\
\hline Number of infarcted segments & 0.25 & $1.28(1.07-1.53)$ & 0.010 & -0.11 & $0.89(0.61-1.32)$ & 0.58 \\
\hline
\end{tabular}

$\mathrm{OR}=$ odds ratio; $\mathrm{Cl}$ = confidence interval; $\mathrm{MI}=$ myocardial infarction; $\mathrm{LVEF}=$ left ventricular ejection fraction.

In patients with undetected acute $\mathrm{MI}$, echocardiography was performed later after admission than in patients in whom acute MI was detected (4.0 [IQR3.0-6.5] vs. 2.0 [IQR1.0-3.0] days, $p=0.001$ ). Accordingly, the probability of detecting an acute MI was inversely related to the delay time between admission and performance of echocardiography ( $b=-0.32, p=0.014$, Table 4$)$. This association was not found in chronic MI.

The probability of accurately detecting acute and chronic $\mathrm{Ml}$ increased with increasing infarct size ( $b=0.16$ for both, $p<0.001$, respectively [Table 4 , Figure $1 A$ and $1 B]$ ), increasing infarct transmurality $(b=0.04, p=0.08$ and $b=0.06, p=0.001$, respectively, 
[Table 4, Figure 2A and 2B]), and increasing number of infarcted segments $(b=0.28$, $p<0.05$ and $b=0.25, p<0.01$, respectively, [Table 4]). All $>75 \%$ transmural chronic $\mathrm{MI}$ were detected (Figure 2B), while one $>75 \%$ transmural, small acute $\mathrm{MI}$ (infarct size $3 \%$ ) remained undetected (Figure $2 \mathrm{~A}$ ).
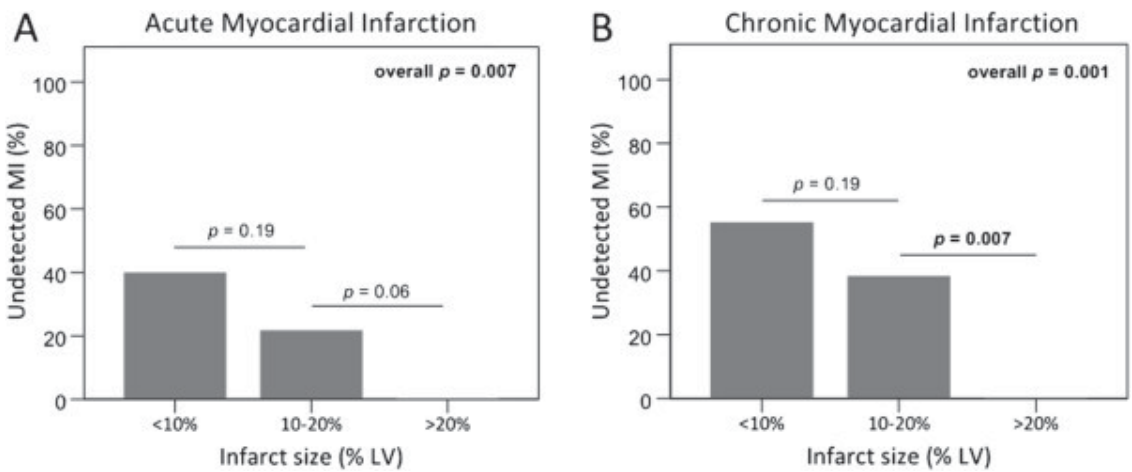

Figure 1: Undetected myocardial infarction in relation to infarct size.

The prevalence of undetected myocardial infarction decreased with increasing infarct size in acute (A) and chronic (B) myocardial infarction.
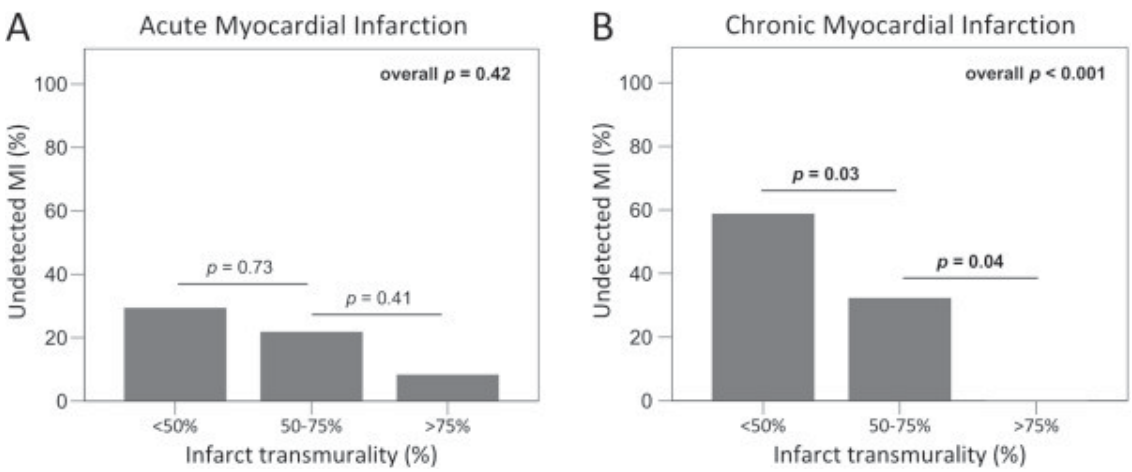

Figure 2: Undetected myocardial infarction in relation to infarct transmurality.

The prevalence of undetected myocardial infarction decreased with increasing infarct transmurality in acute (A) and chronic myocardial infarction (B), and was significant in chronic myocardial infarction only.

Univariate regression analysis revealed that the detection of acute MI was significantly associated with the time point of echocardiography after admission, LVEF, infarct size and segmental extent of infarction (Table 4). Due to the limited number of undetected acute $\mathrm{MI}$, a multivariate logistic regression analysis to evaluate the independent effects of specific infarct characteristics was considered feasible only with respect to chronic MI. The results of a multivariate model, where LVEF, infarct size, infarct transmurality and the number of infarcted segments were entered as independent variables, indicated that only LVEF and infarct size were strongly and independently associated with 
undetected chronic $\mathrm{Ml}(\mathrm{b}=-0.25$ and $\mathrm{b}=0.20, p<0.001$ and $p=0.06$, respectively). Similarly, multivariate regression analysis in the 53 patients with serial echocardiograms resulted in similar associations for both LVEF and infarct size $(b=-0.25$ and $b=0.08)$.

\section{Segmental analysis}

A total of 2370 segments (91\%) were evaluated for SWMA in relation to infarct transmurality by LGE-CMR. A significant positive relationship was found between the severity of SWMA and increasing infarct transmurality $(p<0.001$ for both acute and chronic MI, Figure $3 \mathrm{~A}$ and $\mathrm{B}$ ). XIn acute and chronic $\mathrm{MI}$, median values of infarct transmurality were 0\% (IQRO-8\%) and 0\% (IQRO-7\%) in normokinetic segments, 21\% (IQR3-48) and 25\% (IQR3-58) in hypokinetic segments and 47\% (IQR20-77) and 48\% (IQR10-75) in akinetic segments, respectively.
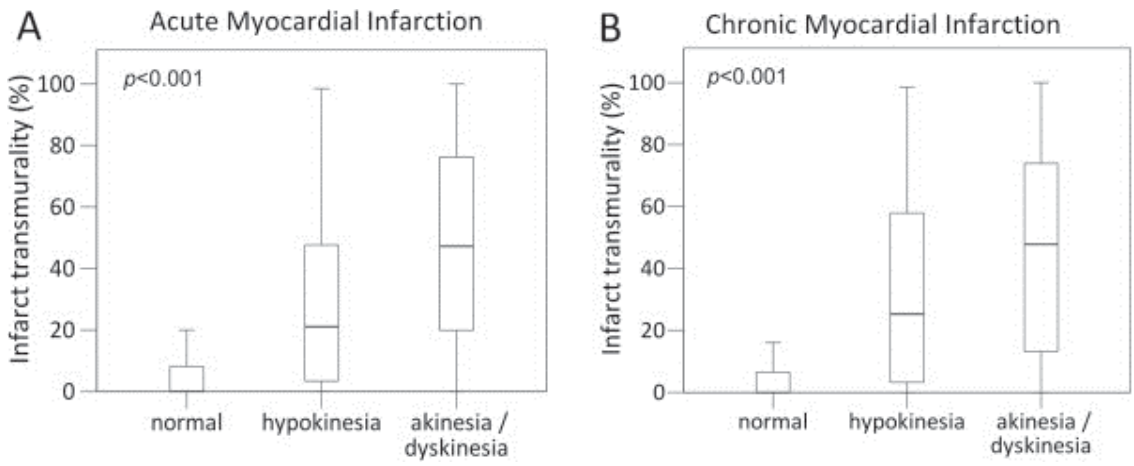

Figure 3: Relationship of echocardiographic segmental wall motion abnormality and infarct transmurality. Box plots illustrating the positive relationship of echocardiographic segmental wall motion abnormality with infarct transmurality in acute (A) and chronic (B) myocardial infarction ( $p<0.001$ for both).

\section{Discussion}

We found that the overall sensitivity of regular transthoracic 2D-echocardiography to detect $\mathrm{Ml}$ is modest. For acute, several days-old MI (first STEMI), its sensitivity was moderate and even lower for chronic, 3 months-old MI. Undetected MI were smaller, less extensive and less transmural and associated with non-anterior localization and higher LVEF.

Accurate detection of $\mathrm{MI}$ is important but can be challenging, especially when symptoms are atypical and electrocardiographic changes are non-specific or absent (non-STEMI). Undetected MI account for at least one-fifth of all infarctions and carry a prognosis as poor as detected MI. ${ }^{20}$ Detecting new loss of myocardium or new SWMA is currently a key part of the universal definition of $\mathrm{Ml}$, and imaging modalities have been 
added to cardiac biomarker testing, ${ }^{16}$ and given a class 1 recommendation in acute coronary syndrome guidelines. ${ }^{21}$

\section{Acute myocardial infarction}

Although a number of studies are being referred to as having shown a high diagnostic accuracy of echocardiography to detect acute $\mathrm{MI}$, several of them were not designed as such or used suboptimal methods to determine infarct size and localization. ${ }^{4}$ Heger et al. investigated patients with Q-wave MI and found SWMA in the entire study population. Q-wave $\mathrm{Ml}$ are predominantly related to infarct size rather than infarct transmurality. ${ }^{22}$ The high incidence of cardiogenic shock, heart failure and deaths, confirms that predominantly patients with large $\mathrm{Ml}$ were studied, positively affecting sensitivity. Of note, 7 out of 44 echocardiograms could not be evaluated and the true sensitivity is therefore lower. ${ }^{5}$ In another study of 90 patients with a first acute MI, SWMA correlated well with the location of $\mathrm{MI}$ on the ECG. All patients had a Q-wave MI, and thus presumably larger MI. Again, only $73 \%$ of echocardiograms could be evaluated. The authors mention the moderate success in obtaining adequate images in the acute setting as an important limitation of echocardiography. ${ }^{6}$ Gibson et al. reported that SWMA were detected in all of 75 patients consecutively admitted with acute MI. However, one-third of patients had a previous MI of whom two-thirds showed SWMA outside the electrocardiographic infarct zone. In this setting, the sensitivity to detect acute MI was overestimated since echocardiography might detect a coexistent large chronic MI while missing a smaller acute MI. In addition, 16 (21\%) patients developed cardiogenic shock of whom 14 (88\%) died, again suggesting that mainly patients with large Ml were studied. ${ }^{7}$ In another study of 43 patients with chest pain and non-diagnostic electrocardiograms, 13 developed a Ml of which 12 (92\%) were detected by echocardiography. However, only patients with good image quality were analyzed and no information was provided on the agreement between SWMA and electrocardiographic location of infarction. ${ }^{8}$ In contrast, two other studies investigating patients with acute non-Q-wave and no previous $\mathrm{Ml}$ found sensitivities of $66 \%$ and $83 \%$, comparable to our findings. ${ }^{9,10}$

The wide variation in sensitivity can be explained by the fact that many studies were small, used different in- and exclusion criteria, or used no or less appropriate reference standards to compare SWMA with. To our knowledge, our study is the largest thus far, specifically addressing the diagnostic accuracy of echocardiography to detect SWMA in comparison to LGE-CMR in a homogenous population of patients with STEMI. We used LGE-CMR as the clinical reference standard to correlate SWMA with. In our study, correct echocardiographic identification of $\mathrm{MI}$ was defined when the location of echocardiographic SWMA matched the infarct region on LGE-CMR images, thereby preventing false positives (i.e. classification of an infarct by echocardiography if SWMA were observed in a remote region [4 out of 141 echocardiograms (2.8\%)]). 


\section{Chronic myocardial infarction}

While most studies have focused on the diagnostic accuracy of echocardiography to detect acute MI, less is known about its usefulness in chronic MI. We found that the sensitivity of echocardiography to detect chronic MI was even lower than for acute MI. This was true in the population as a whole as well as in the cohort of 53 patients with serial echocardiograms. This lower sensitivity can be explained by improvement of contractility of initially "stunned" myocardium several months after successful revascularization. ${ }^{23}$ It also has important clinical implications, because not infrequently, cardiologists are confronted with patients presenting with coincident pathological Q-waves and a normal echocardiogram. In these cases, either the ECG can be false-positive or the echocardiogram false-negative for the diagnosis of chronic MI. To prevent that appropriate therapy is withheld in these patients, LGE-CMR can be applied to definitively rule in or rule out chronic MI. Advanced echocardiographic techniques such as speckle tracking may also play a role to improve diagnostic accuracy.

\section{Relationship with infarct characteristics and LVEF}

MI was more frequently undetected in patients with higher LVEF, smaller, less transmural and less extensive infarctions and when non-anteriorly located. For undetected acute $\mathrm{Ml}$ the relationship with infarct transmurality was less strong than for chronic undetected MI, most likely as a result of "stunning" (Figure 2). The relationship between infarction and contractile function is complex. CMR studies have shown that contractility improves over time in dysfunctional myocardium with $<25 \%$ transmural infarction, ${ }^{13}$ and that the likelihood of functional recovery decreases with increasing infarct transmurality. ${ }^{24}$ The relation of contractile function with infarct transmurality also applies for chronic MI. Blinded observers were unable to detect SWMA in 37\% of infarcted segments, of which $84 \%$ were $<50 \%$ transmurally infarcted. ${ }^{25}$ In our study, all $>75 \%$ transmural chronic MI were correctly identified.

We also found a significant relationship of the severity of echocardiographic SWMA with increasing infarct transmurality (Figure 3), confirming the results of a previous study in 15 autopsied patients in whom ante-mortem echocardiograms were compared with pathology. In that study, all transmurally infarcted segments were akinetic or dyskinetic, and $71 \%$ of subendocardially infarcted segments were either hypokinetic or

normal. ${ }^{26}$ Regardless whether echocardiography or CMR was used, studies have repeatedly shown the relationship of contractility with infarct characteristics. Therefore, MI may remain undetected when the diagnosis is only based on the presence or absence of SWMA.

Echocardiography is a very useful imaging modality in patients with acute chest pain because it can also detect life threatening conditions other than acute $\mathrm{MI}$, such as acute aortic dissection, cardiac tamponade, or pulmonary embolism. It is not our inten- 
tion to bring echocardiography into disrepute, but rather to call for a cautious approach when it is used as a final test to rule out MI.

\section{Limitations}

Although we did not use contrast echocardiography to improve endocardial border definition, ${ }^{27}$ we believe this would not have changed our results. First, image quality was not an important predictor for the detection of both acute and chronic $\mathrm{Ml}$ in our study. Second, a small, subendocardial infarction would still appear normal and therefore remain undetected due to normal contracting neighboring segments ('inverse tethering'). ${ }^{25}$ Echocardiographic strain imaging may have increased the sensitivity to detect $\mathrm{MI}$, as this technique was previously shown to correlate well with the presence and extent of scar on LGE-CMR. ${ }^{28}$ However, accurate strain analysis necessitates additional software and off-line analysis that is less practical in an emergency setting. Whether it may improve the diagnostic accuracy to detect a chronic MI needs further investigation. We cannot exclude that misregistration between echocardiographic and LGE-CMR images affected our results, although segments were visualized in multiple views in the majority of patients.

We investigated a homogenous study population, albeit with a modest sample size. Echocardiograms were not available in all patients, and this may have affected our results. At the time of the first echocardiogram (median 2 days, IQR1-4), SWMA might have resolved in some patients, lowering sensitivity. However, the process of myocardial stunning after acute $\mathrm{Ml}$ generally lasts days to weeks. ${ }^{29}$ Although automatic quantification of infarct size and transmurality might have improved accuracy, manual adjustment of contours often remains necessary. Furthermore, no consensus exists on what signal intensity thresholds should be used.

\section{Conclusions}

The sensitivity of regular echocardiography to detect SWMA in patients initially presenting with STEMI is moderate in the acute phase and even lower in the chronic phase. Undetected $\mathrm{Ml}$ were smaller, less extensive and less transmural, and associated with non-anterior localization and higher LVEF. Although echocardiography is recommended by current acute coronary syndrome guidelines, excluding Ml solely based on wall motion should be done cautiously. When clinical suspicion remains high and all other diagnostic tests are inconclusive, LGE-CMR can be applied. 


\section{References}

1. Roger VL, Go AS, Lloyd-Jones DM, Adams RJ, Berry JD, Brown TM, Carnethon MR, Dai S, de Simone G, Ford ES. Heart disease and stroke statistics-2011 update: a report from the American Heart Association. Circulation. 2011;15:e18-e209

2. Kim HW, Klem I, Shah DJ, Wu E, Meyers SN, Parker MA, Crowley AL, Bonow RO, Judd RM, Kim RJ. Unrecognized non-Q-wave myocardial infarction: prevalence and prognostic significance in patients with suspected coronary disease. PLoS Med. 2009;15:e1000057.

3. Kerber RE, Abboud FM. Echocardiographic detection of regional myocardial infarction: an experimental study. Circulation. 1973;15:997-1005.

4. Kontos MC. Role of echocardiography in the emergency department for identifying patients with myocardial infarction and ischemia. Echocardiography. 1999;15:193-205.

5. Heger JJ, Weyman AE, Wann LS, Dillon JC, Feigenbaum H. Cross-sectional echocardiography in acute myocardial infarction: detection and localization of regional left ventricular asynergy. Circulation. 1979;15:531-538.

6. Visser CA, Lie KI, Kan G, Meltzer R, Durrer D. Detection and quantification of acute, isolated myocardial infarction by two dimensional echocardiography. Am J Cardiol. 1981;15:1020-1025.

7. Gibson RS, Bishop HL, Stamm RB, Crampton RS, Beller GA, Martin RP. Value of early two dimensional echocardiography in patients with acute myocardial infarction. Am J Cardiol. 1982;15:1110-1119.

8. Peels CH, Visser CA, Kupper AJ, Visser FC, Roos JP. Usefulness of two-dimensional echocardiography for immediate detection of myocardial ischemia in the emergency room. Am J Cardiol. 1990;15:687-691.

9. Loh IK, Charuzi Y, Beeder C, Marshall LA, Ginsburg JH. Early diagnosis of nontransmural myocardial infarction by two-dimensional echocardiography. Am Heart J. 1982;15:963-968.

10. Arvan S, Varat MA. Two-dimensional echocardiography versus surface electrocardiography for the diagnosis of acute non-Q wave myocardial infarction. Am Heart J. 1985;15:44-49.

11. Kisslo JA, Robertson D, Gilbert BW, von Ramm O, Behar VS. A comparison of real-time, two dimensional echocardiography and cineangiography in detecting left ventricular asynergy. Circulation. 1977;15:134141.

12. Weyman AE, Peskoe SM, Williams ES, Dillon JC, Feigenbaum H. Detection of left ventricular aneurysms by cross-sectional echocardiography. Circulation. 1976;15:936-944.

13. Choi KM, Kim RJ, Gubernikoff G, Vargas JD, Parker M, Judd RM. Transmural extent of acute myocardial infarction predicts long-term improvement in contractile function. Circulation. 2001;15:1101-1107.

14. Lieberman AN, Weiss JL, Jugdutt BI, Becker LC, Bulkley BH, Garrison JG, Hutchins GM, Kallman CA, Weisfeldt ML. Two-dimensional echocardiography and infarct size: relationship of regional wall motion and thickening to the extent of myocardial infarction in the dog. Circulation. 1981;15:739-746.

15. Bekkers SC, Backes WH, Kim RJ, Snoep G, Gorgels AP, Passos VL, Waltenberger J, Crijns HJ, Schalla S. Detection and characteristics of microvascular obstruction in reperfused acute myocardial infarction using an optimized protocol for contrast-enhanced cardiovascular magnetic resonance imaging. Eur Radiol. 2009;15:2904-2912.

16. Thygesen K, Alpert JS, White HD, Jaffe AS, Apple FS, Galvani M, Katus HA, Newby LK, Ravkilde J, Chaitman B. Universal definition of myocardial infarction. Circulation. 2007;15:2634-2653.

17. Picard MH, Adams D, Bierig SM, Dent JM, Douglas PS, Gillam LD, Keller AM, Malenka DJ, Masoudi FA, McCulloch M. American Society of Echocardiography recommendations for quality echocardiography laboratory operations. J Am Soc Echocardiogr. 2011;15:1-10.

18. Cerqueira MD, Weissman NJ, Dilsizian V, Jacobs AK, Kaul S, Laskey WK, Pennell DJ, Rumberger JA, Ryan T, Verani MS. Standardized myocardial segmentation and nomenclature for tomographic imaging of the heart: a statement for healthcare professionals from the cardiac imaging committee of the council on clinical cardiology of the American heart association. Circulation. 2002;15:539-542.

19. Harrell FE. Regression Modeling Strategies. New York: Springer; 2001.

20. Sheifer SE, Manolio TA, Gersh BJ. Unrecognized myocardial infarction. Ann Intern Med. 2001;15:801-811. 
21. Hamm CW, Bassand JP, Agewall S, Bax J, Boersma E, Bueno H, Caso P, Dudek D, Gielen S, Huber K. ESC guidelines for the management of acute coronary syndromes in patients presenting without persistent ST-segment elevation: the task force for the management of acute coronary syndromes (ACS) in patients presenting without persistent ST-segment elevation of the European society of cardiology (ESC) Eur Heart J. 2011;15:2999-3054.

22. Moon JC, De Arenaza DP, Elkington AG, Taneja AK, John AS, Wang D, Janardhanan R, Senior R, Lahiri A, Poole-Wilson PA, Pennell DJ. The pathologic basis of $\mathrm{Q}$-wave and non-Q-wave myocardial infarction: a cardiovascular magnetic resonance study. J Am Coll Cardiol. 2004;15:554-560.

23. Bolli R. Mechanism of myocardial "stunning" Circulation. 1990;15:723-738.

24. Beek AM, Kuhl HP, Bondarenko O, Twisk JW, Hofman MB, van Dockum WG, Visser CA, van Rossum AC. Delayed contrast-enhanced magnetic resonance imaging for the prediction of regional functional improvement after acute myocardial infarction. J Am Coll Cardiol. 2003;15:895-901.

25. Mahrholdt H, Wagner A, Parker M, Regenfus M, Fieno DS, Bonow RO, Kim RJ, Judd RM. Relationship of contractile function to transmural extent of infarction in patients with chronic coronary artery disease. J Am Coll Cardiol. 2003;15:505-512.

26. Weiss JL, Bulkley BH, Hutchins GM, Mason SJ. Two-dimensional echocardiographic recognition of myocardial injury in man: comparison with postmortem studies. Circulation. 1981;15:401-408.

27. Lonnebakken MT, Staal EM, Nordrehaug JE, Gerdts E. Usefulness of contrast echocardiography for predicting the severity of angiographic coronary disease in non-ST-elevation myocardial infarction. Am J Cardiol. 2011;15:1262-1267

28. Roes SD, Mollema SA, Lamb HJ, van der Wall EE, de Roos A, Bax JJ. Validation of echocardiographic twodimensional speckle tracking longitudinal strain imaging for viability assessment in patients with chronic ischemic left ventricular dysfunction and comparison with contrast-enhanced magnetic resonance imaging. Am J Cardiol. 2009;15:312-317.

29. Braunwald E, Kloner RA. The stunned myocardium: prolonged, postischemic ventricular dysfunction. Circulation. 1982;15:1146-1149. 



\section{Non-invasive assessment of microvascular dysfunction in patients with cardiac syndrome $X$}

C. Jaarsma, J. van Haare, H. Vink, S.C. Bekkers, B.D. van Rooijen, W.H. Backes, J. Wildberger, H.J. Crijns, J. van Teeffelen, S. Schalla.

Submitted. 


\section{Abstract}

Objectives: We aimed to evaluate the microvascular function in cardiac syndrome $X$ (CSX) patients by assessing 1) the endothelial glycocalyx barrier properties using sublingual microscopy, and 2) the myocardial perfusion reserve using cardiovascular magnetic resonance $(\mathrm{CMR})$ imaging.

Background: It has been suggested that CSX may be related to coronary microvascular dysfunction.

Methods and results: Sublingual microscopy was performed in 13 CSX patients (angina pectoris, ST-depression on treadmill testing, normal coronary angiogram) and compared with 2 control groups of 13 volunteers and 14 patients with known obstructive coronary artery disease (CAD). To test the glycocalyx-mediated microvascular responsiveness, the erythrocyte perfused boundary region (PBR) was assessed at baseline and after nitroglycerin challenge. The baseline PBR of CSX patients was similar to controls with CAD ( $p=0.72)$, and larger than in volunteers $(p=0.02)$. Only the volunteers demonstrated a significant increase in PBR after nitroglycerin $(p=0.03)$. In the 13 CSX patients, adenosine stress CMR perfusion imaging was performed. Although a significant increase in myocardial perfusion was observed in both the subendocardium and subepicardium during stress, the subendocardial perfusion reserve was significantly lower $(p=0.02)$. The PBR responsiveness of the sublingual microvasculature showed a strong correlation with the transmural myocardial perfusion reserve $(r=0.86, p<0.001)$.

Conclusions: CSX patients can be characterized by microvascular glycocalyx dysfunction using sublingual microscopy. The strong correlation between sublingual PBR responsiveness and myocardial perfusion reserve indicates that the glycocalyx plays an important role in the regulation of microvascular volume for myocardial perfusion and supports the concept of impaired glycocalyx barrier properties in CSX. 


\section{Introduction}

Patients with chest pain in the absence of obstructive coronary artery disease (CAD) remain a challenge for physicians, since the underlying pathophysiology and appropriate treatment are currently not fully understood..$^{1-3}$ Within this patient population, cardiac syndrome $X(C S X)$ is a well defined entity that is characterized by symptoms of typical angina pectoris on exertion with corresponding ST-depression on electrocardiography, despite normal coronary arteries on invasive coronary angiography. ${ }^{4}$ Although there is currently no consensus on the pathophysiologic basis of CSX, it has been proposed that coronary microvascular dysfunction plays an important role. ${ }^{5,6}$ However, in contrast to the epicardial coronary vasculature, direct assessment of coronary microvascular dysfunction is difficult. Cardiovascular magnetic resonance (CMR) perfusion imaging has the ability to detect myocardial ischemia non-invasively with a good spatial resolution. ${ }^{7}$ However, previous studies using CMR perfusion imaging to detect myocardial ischemia as a marker for coronary microvascular dysfunction in patients with CSX have reported conflicting results. ${ }^{8-11}$ Alternatively, the microcirculation can be directly assessed in easily accessible regions such as the sublingual circulation by novel noninvasive imaging techniques. Recent experimental and clinical studies using intravital microscopy of the sublingual microvasculature have indicated that evaluation of damage to the endothelial glycocalyx may reflect microvascular dysfunction. ${ }^{12-16}$ The endothelial glycocalyx is a cell-hindering layer on the luminal side of blood vessels that plays an important role in the protection of the vascular wall against atherogenic stimuli (Figure 1). ${ }^{17}$ Perturbation of this protective layer has been demonstrated in healthy volunteers during acute hyperglycemia and in patients with vascular disease, allowing deeper cell penetration towards the endothelium leading to an increase in the erythrocyte perfused boundary region (PBR). ${ }^{12-15,17,18}$

The current study aimed to investigate the microvascular function of patients with CSX using these non-invasive imaging techniques. Therefore, we assessed the baseline PBR and its responsiveness as a marker for glycocalyx-mediated microvascular function using sublingual microscopy in a well described homogeneous population of CSX patients, in comparison with 2 control groups (i.e. subjects with and without known obstructive coronary artery disease [CAD]). Additionally, we evaluated the myocardial perfusion reserve of the CSX patients with high spatial resolution adenosine stress CMR perfusion imaging at 3.0 Tesla. 


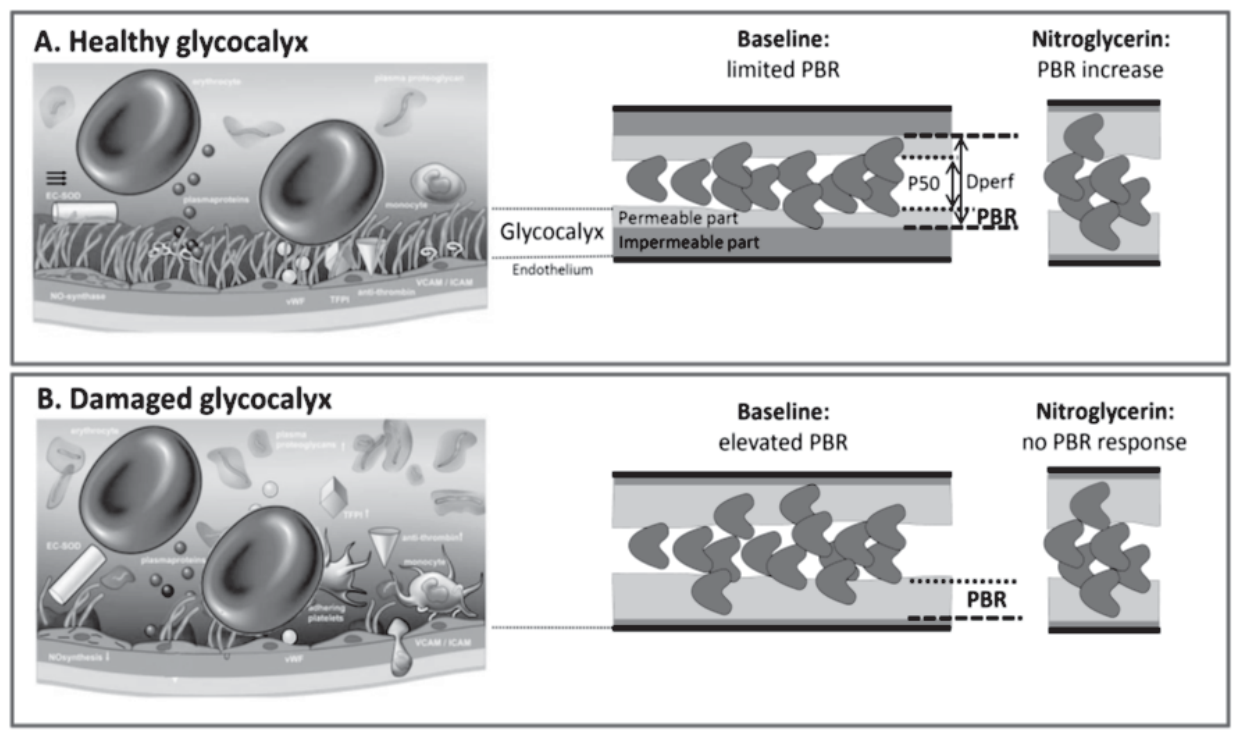

Figure 1: Portrayal of the endothelial glycocalyx and its relation to the perfused boundary region (PBR) in a microvessel.

A. Left: A healthy glycocalyx limits the accessibility of blood-borne lipids and proteins and forms a barrier for adhesion of platelets and inflammatory cells to the vascular wall. It is also involved in mechanosensing and transduction of hemodynamic stimuli to the endothelium, thereby regulating the production of amongst others nitric oxide.

The cartoons in the center and right schematically illustrate the perfused boundary region (PBR) in relation to the glycocalyx barrier properties in a blood vessel. The PBR is the cell-poor layer which results from the phase separation between the flowing erythrocytes and plasma. It covers the cell-permeable part of the glycocalyx, to which erythrocytes have limited access, while the cell-impermeable part cannot be accessed at all. In a healthy vessel, outward movement of the erythrocytes under baseline conditions is restricted by the relatively thick cell-impermeable glycocalyx barrier, resulting in a small PBR. Nitroglycerin, however, is anticipated to robustly increase the PBR in this vessel by impairing this barrier. The PBR is the main readout parameter of the sublingual imaging, and calculated from the median erythrocyte column width (P50) and outer diameter of erythrocyte perfused lumen (Dperf); this is further explained in Figure 2. Modified from (15) and (36), with permission.

B. Left: Damage to the glycocalyx has been associated with all features of a malfunctioning microcirculation: endothelial activation, vascular leakage and a diminished NO bioavailability. A damaged glycocalyx is associated with a reduction of the cell-impermeable glycocalyx part allowing the outer edge of the erythrocyte perfused lumen to move in sideward direction towards the endothelium, resulting in an increase in PBR at baseline already and the absence of a PBR response to nitroglycerin (center and right).

\section{Methods}

\section{Study population}

Consecutive patients with CSX were prospectively enrolled at our institution between 2011 and 2012. The CSX patients had typical exercise-induced angina pectoris and cor- 
responding ischemic ST-changes on electrocardiography (defined as $\geq 0.1 \mathrm{mV}$ horizontal or down sloping ST-segment depression $80 \mathrm{~ms}$ after the J point). They all had previously undergone invasive coronary angiography, showing normal coronary arteries $(n=8)$ or minimal vessel wall irregularities (i.e. $<25 \%$ stenosis, $n=5$ ). Patients younger than 18 years and patients with contraindications for either CMR (e.g. metallic implants, pacemaker) or adenosine (e.g. atrioventricular conduction abnormalities, severe asthma) were excluded. Of the 16 eligible CSX patients, 13 gave informed consent and completed the entire study protocol.

In addition to these 13 CSX patients, 13 volunteers without a history of chest pain or CAD and 14 patients with known obstructive CAD (examined approximately one hour before their scheduled percutaneous revascularization) of similar age and sex distribution were recruited during the study period to serve as control groups for the sublingual microscopy. Subjects (i.e. CSX patients, volunteers, or CAD patients) with a history of (medically) controlled mild hypertension or hypercholesterolemia were not excluded. Our local Institutional Review Board only approved adenosine stress CMR perfusion imaging in the patients with CSX, since there was no myocardial ischemia suspected in the volunteers, and already known obstructive CAD with ischemia in the patients with CAD.

\section{Sublingual microvasculature imaging}

All 40 study subjects (i.e. 13 CSX patients, 13 volunteers, and 14 CAD patients) underwent imaging of the sublingual microvasculature using a handheld sidestream darkfield microscan videomicroscope (MicroVision Medical Inc., Wallingford, PA). Analyses of glycocalyx barrier properties were performed by calculating the PBR using GlycoCheck Glycocalyx Measurement Software (GlycoCheck, Maastricht, the Netherlands). The measurements were performed after an overnight fast, during which the study subjects were also asked to refrain from smoking. Prescribed medication was continued. Each subject underwent 2 baseline measurements and 2 measurements performed starting 2 minutes after sublingual administration of nitroglycerin $(0.4 \mathrm{mg}$ spray dose). Under physiological conditions, nitroglycerin is anticipated to rapidly increase the PBR by modulating the barrier properties of the glycocalyx, but this effect is diminished in case of glycocalyx degradation in diseased states. ${ }^{16,19}$ Thus, an increased baseline PBR as well as an impaired PBR response to nitroglycerin were considered to reflect microvascular dysfunction as a result of perturbation of the endothelial glycocalyx.

The techniques and reproducibility of imaging and analysis of the glycocalyx barrier properties have been described previously. ${ }^{14-16}$ The parameters of interest for the analysis are schematically depicted in Figure 1, while a detailed description of both the sublingual imaging technique and the calculation of the PBR are presented in Figure 2. For each measurement in a subject, the calculated PBR values were classified according to

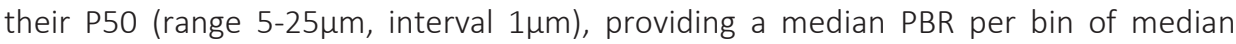


erythrocyte column width, from which the average PBR was calculated to provide a single PBR value per subject per measurement. The baseline PBR was taken as the average of both baseline measurements, while for the nitroglycerin PBR the highest PBR value in either one of the two measurements after nitroglycerin challenge was taken. The PBR response was calculated by subtracting the baseline PBR from the nitroglycerin PBR.

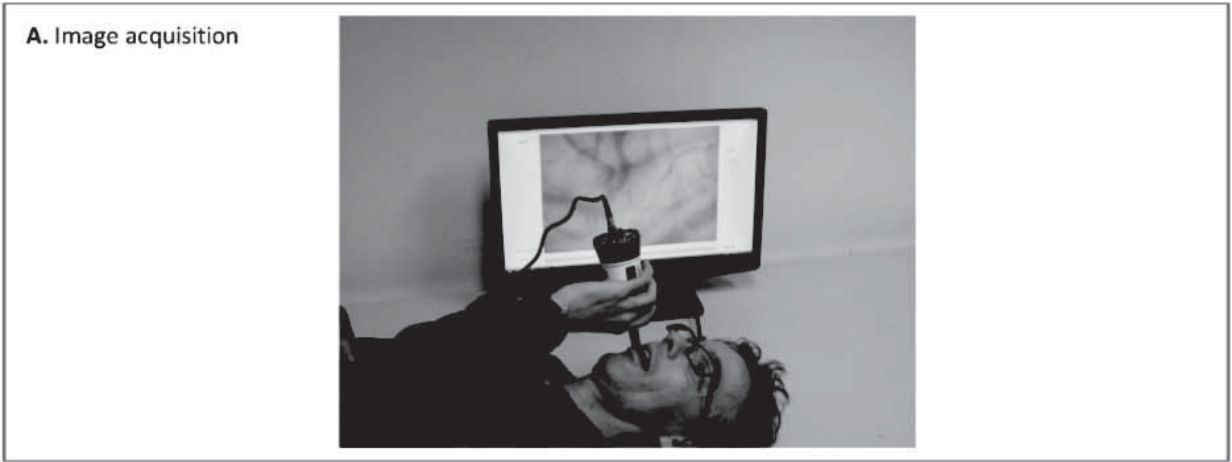

B. Image recording and analysis

Step 1. Collected movie frame

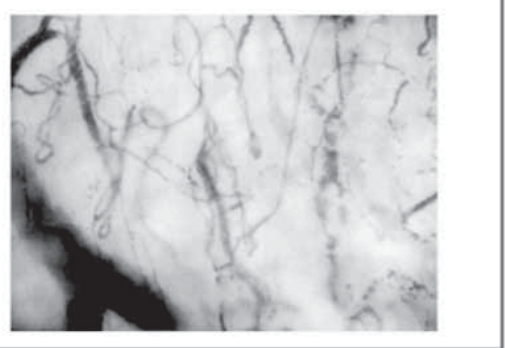

Step 3. For each vessel segment: determination of erythrocyte column width for 40 frames

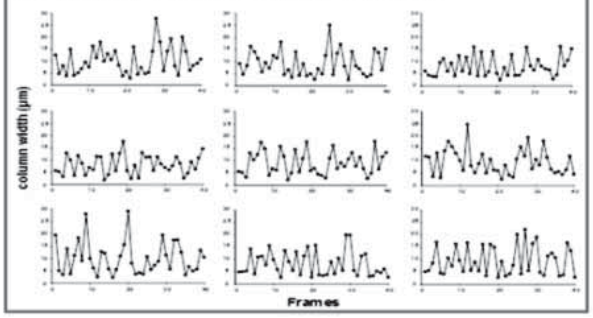

Step 2. Vessel segments defined

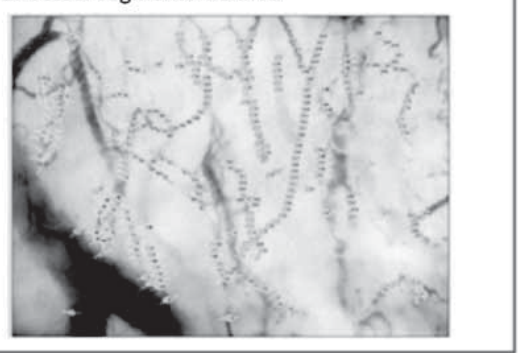

Step 4. Calculation of PBR from cumulative distribution

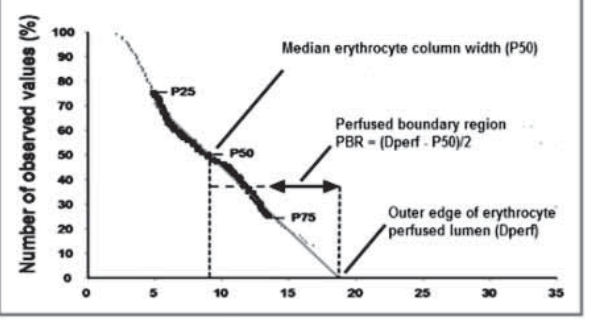

Figure 2: Imaging of sublingual microcirculation and calculation of the perfused boundary region (PBR) as reflection a of glycocalyx barrier properties.

A: The sublingual microvasculature was imaged with the subject in a supine position using a handheld sidestream darkfield microscan videomicroscope (MicroVision Medical Inc., Wallingford, PA). This microscope is equipped with a $5 \mathrm{X}$ magnifying objective lens system-containing probe (numerical aperture: 0.2), imaging the 
erythrocytes in the tissue-embedded microcirculation using green pulsed LED ring illumination.(37) The region chosen for measurement was the central sublingual area; pressure on the tissue was avoided to ensure normal flow.

B: Automated image recording and analysis were performed using GlycoCheck Glycocalyx Measurement Software (GlycoCheck, Maastricht, the Netherlands). By online monitoring of tissue motion, illumination intensity, and focus level of the sublingual microvasculature, the recording of a sufficient number of highquality videos was guaranteed by the software. Sublingual images were acquired at a frame rate of 23 per second with a final on-screen magnification of 325x. Video acquisition automatically started when image quality was within an acceptable range and automatically stopped when a sufficient number of videos had been collected for analysis; per measurement approximately 10 movies of 40 consecutive frames were recorded, giving a total recording time of $2-5 \mathrm{~min}$. Image analysis involved four steps which in the end resulted in the determination of the PBR at multiple sites along the microvasculature. Analysis automatically started during the recording stage (step 1). The first frame was used to automatically identify all available microvessels with a diameter between 5 and $25 \mu \mathrm{m}$. Every $10 \mu \mathrm{m}$ along the length of the detected vasculature marker lines were placed perpendicular to the vessel direction (step 2). Each line represented a single vessel segment, for which 21 parallel intensity profiles (every $\pm 0.5 \mu \mathrm{m}$ ) were obtained; within each intensity profile the erythrocyte column width was calculated from the full width half maximum. This was done for all 40 consecutive frames in a movie (step 3; note that just 9 representative RBC column width tracings and not the total number of 21 for a particular vessel segment are shown). In this way, a total of 840 erythrocyte column width measurements per vessel segment were generated, for which a cumulative distribution was calculated (step 4). From this distribution, the median erythrocyte column width (P50) was taken while the outer edges of the erythrocyte perfused lumen (Dperf) were derived using linear extrapolation between the 25th and 75th percentile values (step 4). Subsequently, the PBR was calculated from the distance of the median erythrocyte column width to the outer edge of the erythrocyte perfused lumen; i.e. PBR $=($ Dperf - P50) $/ 2$.

\section{Cardiovascular magnetic resonance imaging}

Following the sublingual microvascular imaging, the 13 CSX patients subsequently underwent CMR imaging on a 3.0 Tesla MR system (Achieva, Philips Healthcare, Best, the Netherlands) equipped with a cardiac software package and a SENSE 6 element cardiac array coil. These patients were asked to refrain from caffeine and beta-blockers the morning of the study. The median time from invasive coronary angiography to CMR imaging was 4 weeks (range 0-19 weeks).

All images were acquired with electrocardiographic triggering and during expiratory breath hold. The protocol included standard cine (steady-state free precession) and late gadolinium enhancement imaging. None of the patients had evidence of regional wall motion abnormalities on cine images or hyperenhancement on late gadolinium enhancement images. Stress perfusion imaging was performed during intravenous adenosine at a dose of $140 \mu \mathrm{g} / \mathrm{kg} / \mathrm{min}$ during heart rate and blood pressure monitoring. The pulse-sequence used for perfusion imaging has been described in detail previously.(20) Perfusion data were acquired in 3 slices in short-axis view using a saturation recovery gradient echo pulse sequence accelerated with $k$-t SENSE (TR/TE 3.2/1.54ms, flip angle $15^{\circ}$, saturation prepulse delay $110 \mathrm{~ms}$, acquisition window $140 \mathrm{~ms}$, slice thickness $8 \mathrm{~mm}$, $k$ - $t$ factor of 5 with $11 k$ - $t$ interleaved training profiles, effective acceleration 3.8). After 
4 minutes of adenosine infusion, a bolus of $0.05 \mathrm{mmol} / \mathrm{kg}$ body weight gadobutrol (Gadovist, Bayer-Schering, Germany) was administered. Rest perfusion was performed after approximately 15 minutes, using the same bolus technique.

Two independent observers analyzed the CMR perfusion images qualitatively, using commercially available software (CAAS MRV 3.0, Pie Medical Imaging, Maastricht, the Netherlands). Any discrepancies were resolved in consensus. The image quality was scored as 0 = poor, 1 = average, and 2 = excellent. Perfusion defects were deemed present if a subendocardial or transmural delay of contrast enhancement was seen relative to a remote region and persisted for more than 5 frames. Initial delay of subendocardial contrast enhancement, which disappeared after approximately 5 heartbeats, was considered an artifact. ${ }^{9,11}$ For semi-quantitative analysis, the endocardial and epicardial contours were traced manually and automatically divided into the 16 segments of the basal, mid, and apical slices according to the AHA 17-segment model. ${ }^{21}$ These segments were further subdivided into subendocardial and subepicardial segments (i.e. the inner and outer $50 \%$ of each segment, respectively). Additionally, a region of interest was drawn in the cavity of the left ventricle to record an arterial input function. Quantitative analysis of the tissue enhancement curves was performed using Matlab (The MathWorks Inc., Natick, MA). Signal intensity was converted to signal enhancement by dividing by the mean pre-contrast signal. Perfusion was quantified using Patlak Plot analysis, which assumes that there is no contrast efflux from the tissue into the vasculature. ${ }^{22}$ As such, it assumes a linear relationship between enhancement in the myocardium $\left(C_{\text {tis }}\right)$ and the time integral of the enhancement in the blood pool $\left(C_{b l}\right)$ (equation 1$)$. The slope of the curve $\mathrm{K}_{\mathrm{tr}}$ can be used as a measure for perfusion of the myocardium. ${ }^{23,24}$

$$
C_{t i s}(t)=K_{t r} \int_{0}^{t} C_{b l}(t) d t
$$

To improve the goodness of fit of $K_{t r}$, the enhancement curves were manually selected to include the start of the arrival of the contrast agent in the left ventricle up to the maximum enhancement in the myocardium before fitting the model. The myocardial perfusion reserve was calculated by dividing the $K_{t r}$ of the stress perfusion by the $K_{t r}$ of the perfusion in rest.

\section{Statistical analysis}

Continuous variables with normally distributed data are expressed as mean \pm standard deviation (SD), otherwise as median with the interquartile range (IQR). Categorical data are expressed as frequencies with percentages. Differences in baseline characteristics between the study groups were evaluated using one-way ANOVA (for continuous data) and the Chi-square or Fisher's exact test (for categorical data). For comparisons within subjects (e.g. rest versus stress) the Wilcoxon test for paired samples was used. The Mann Whitney $U$ test was used to compare data between study groups. No adjustment for multiple comparisons were made. The Spearman correlation coefficient was used to 
evaluate the correlation between the sublingual PBR measurements and the CMR derived MPRI ratio. SPSS version 17.0 (SPSS Inc., Chicago, IL) was used for all statistical analyses. A two-tailed value of $p<0.05$ was considered statistically significant.

\section{Results}

\section{Baseline characteristics}

Characteristics of the study subjects are presented in Table 1. The three study groups demonstrated similar distributions of age and sex. The CSX patients and the control subjects with CAD more often had cardiovascular risk factors, especially smoking or a family history of CAD, than the volunteers.

Table 1: Baseline characteristics of the study population.

\begin{tabular}{lcccc}
\hline & CSX & \multicolumn{2}{c}{ Controls } & P \\
\cline { 3 - 4 } & & Volunteers & CAD & \\
\hline & $N=13$ & $N=13$ & $N=14$ & 0.38 \\
Age, years & $65 \pm 9$ & $63 \pm 10$ & $68 \pm 8$ & 0.52 \\
Male (\%) & $6(46)$ & $9(69)$ & $9(64)$ & 0.02 \\
BMI, kg/m ${ }^{2}$ & $26 \pm 3$ & $25 \pm 3$ & $28 \pm 3$ & 0.34 \\
Diabetes mellitus (\%) & $2(15)$ & $0(0)$ & $3(21)$ & $<0.01$ \\
History of smoking (\%) & $10(77)$ & $4(31)$ & $2(14)$ & 1.00 \\
Current (\%) & $0(0)$ & $1(8)$ & $7(50)$ & 0.78 \\
Hypertension (\%) & $5(38)$ & $5(38)$ & $8(57)$ & 0.16 \\
Hypercholesterolemia (\%) & $6(46)$ & $3(23)$ & $8(57)$ & 0.04 \\
Family history of CAD (\%) & $8(62)$ & $2(15)$ & \\
\hline
\end{tabular}

Values are presented as mean \pm standard deviation or proportions (\%), when appropriate.

$\mathrm{BMI}=$ body mass index; $\mathrm{CAD}=$ coronary artery disease; $\mathrm{CSX}=$ cardiac syndrome $\mathrm{X}$.

\section{Sublingual microvasculature imaging}

The median baseline PBR of patients with CSX was similar to that in the controls with

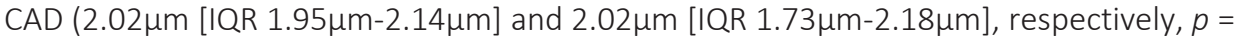
$0.72)$, but significantly larger compared with the volunteers $(1.90 \mu \mathrm{m}$ [IQR $1.77 \mu \mathrm{m}$ $2.02 \mu \mathrm{m}], p=0.02$, Figure $3 \mathrm{~A})$. This suggests that, compared with volunteers, both CSX and CAD patients showed a deeper erythrocyte penetration into the glycocalyx because of a decrease in its integrity. Figure 3B shows that the maximum PBR after NTG challenge did not differ significantly between any of the groups $(2.09 \mu \mathrm{m}$ [IQR $1.85 \mu \mathrm{m}$ $2.16 \mu \mathrm{m}$ ], $1.96 \mu \mathrm{m}$ [IQR $1.85 \mu \mathrm{m}-2.14 \mu \mathrm{m}$ ], and $2.12 \mu \mathrm{m}$ [IQR $2.01 \mu \mathrm{m}-2.33 \mu \mathrm{m}$ ] for CSX patients, volunteers, and controls with $C A D$, respectively). Hence, the nitroglycerin 
challenge caused only a minimal increase in median PBR in CSX patients and control subjects with $\operatorname{CAD}(p=0.70$ and $p=0.18$, respectively), whereas the volunteers demonstrated a significant increase in their PBR upon nitroglycerin challenge. Comparisons between groups revealed that the median PBR response to nitroglycerin was significantly smaller in CSX patients than in the volunteers ( $p=0.03$, Figure 3C).

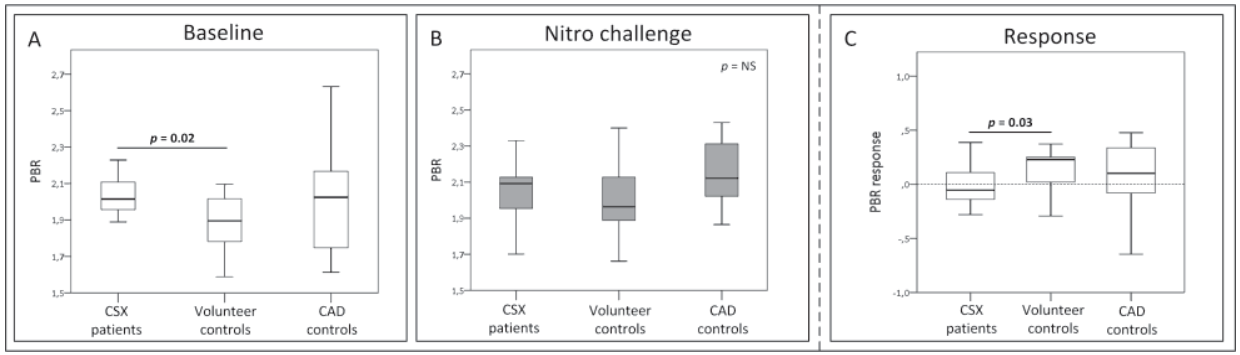

Figure 3: Measurements of perfused boundary region with sublingual microscopy.

Results of sublingual perfused boundary region (PBR) measurements in patients with cardiac syndrome $X$ (CSX), volunteers, and controls with obstructive coronary artery disease (CAD).

Baseline PBR measurements (panel A) in CSX patients were significantly higher than in volunteers. After nitroglycerin challenge (panel B), all groups had a comparable PBR. Panel C represents the PBR response (i.e. the difference between panel $A$ and $B$ ). Only the volunteers demonstrated a significant increase in PBR after nitroglycerin challenge $(p=0.03)$, which was a significantly larger PBR response than observed in the CSX patients (Panel C).

\section{CMR perfusion imaging in patients with cardiac syndrome $X$}

During adenosine vasodilator stress, the 13 CSX patients undergoing CMR perfusion imaging demonstrated an increase in mean heart rate from $69 \pm 12$ to $97 \pm 16$ beats per minute. At peak stress, both the mean systolic and diastolic blood pressure decreased slightly from $138 \pm 14$ to $130 \pm 17 \mathrm{mmHg}$ and $78 \pm 9$ to $73 \pm 7 \mathrm{mmHg}$, respectively. No relevant side effects were observed during adenosine infusion, although some patients reported transient chest pain $(N=5)$, flushing $(N=5)$, or dyspnea $(N=1)$.

None of the patients had evidence of perfusion defects on visual perfusion analysis. Semi-quantitative perfusion analysis showed that the myocardial perfusion index (MPI) for the entire transmural extent increased significantly during adenosine stress $(0.054$ [IQR 0.040-0.068] to 0.064 [IQR 0.060-0.103], $p<0.01$ ), with an MPRI of 1.35 (IQR 1.102.28). Similarly, the MPI increased in subendocardial segments from 0.055 (IQR 0.0450.077 ) to 0.063 (IQR 0.057-0.115), $p=0.03$, and in subepicardial segments from 0.046 (IQR 0.037-0.058) to 0.070 (IQR 0.062-0.095), $p=0.001$. However, the myocardial perfusion reserve of subendocardial segments was significantly lower than that of subepicardial segments (i.e. an MPRI of 1.23 [IQR 0.99-2.12] vs. 1.53 [IQR 1.27-2.42], $p=0.02$, Figure 4). 


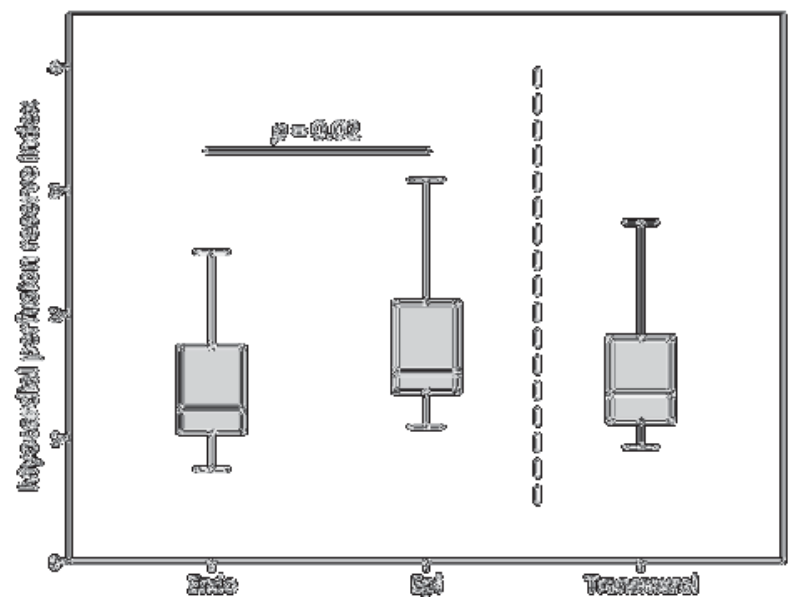

Figure 4: Cardiovascular magnetic resonance perfusion measurements in patients with cardiac syndrome $X$.

The myocardial perfusion reserve index (MPRI) in endocardial segments was significantly lower in comparison to epicardial segments $(p=0.02)$.

A value of $<0.72$ has been proposed in a previous publication (performed at 1.5 Tesla using gadopentate dimeglumine contrast medium) as a possible cut-off value for the ratio of subendocardial-to-subepicardial MPRI to distinguish between CSX patients and healthy controls.(8) In the current study, the median subendocardial-to-subepicardial MPRI ratio was 0.81 (IQR 0.71-0.90), and 3 patients demonstrated a ratio of $<0.72$ (i.e. $0.64,0.65$, and 0.68$)$.

\section{Correlation sublingual microvascular function and myocardial perfusion reserve}

To explore the correlation between the sublingual assessment of glycocalyx-associated microvascular function and myocardial perfusion measurements by CMR, we performed a correlation analysis for the PBR-response and the transmural MPRI. An initial analysis demonstrated a correlation coefficient of $0.46(p=0.11)$. The exclusion of the only CSX patient with poor image quality on CMR perfusion imaging revealed a strong correlation between PBR-increase and MPRI ( $r=0.86, p<0.001$, Figure 5). The results of the other analyses based on CMR perfusion measurements were not significantly altered by excluding this patient. (e.g. the comparison between endocardial and epicardial MPRI remained statistically significant with $p=0.03$ ). 


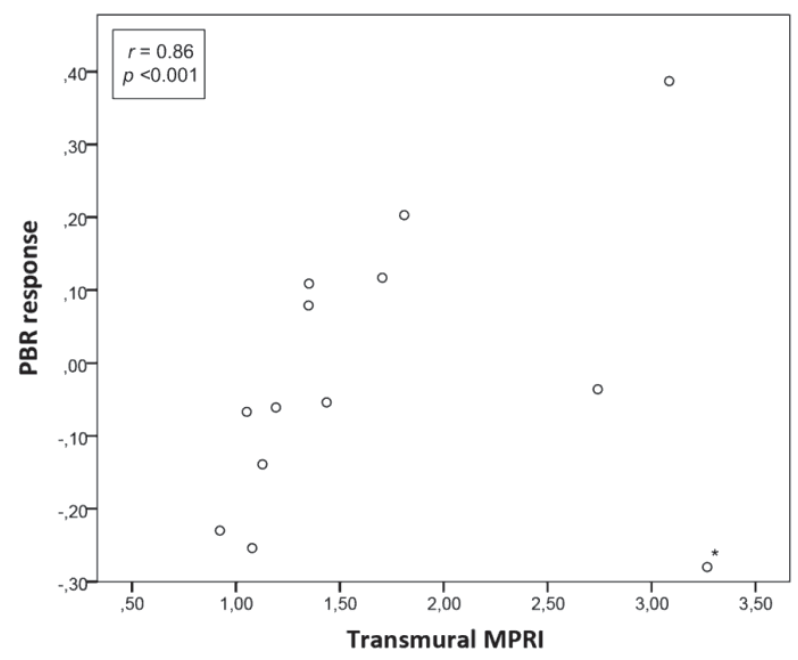

Figure 5: Correlation between the perfused boundary region response and transmural myocardial perfusion reserve index in patients with cardiac syndrome $X$.

Scatter plot illustrating a good correlation between the perfused boundary region (PBR) response as evaluated by sublingual microscopy and the cardiovascular magnetic resonance (CMR) derived transmural myocardial perfusion reserve index (MPRI) in patients with cardiac syndrome $X$.

* Outlier excluded from analysis due to poor image quality on CMR.

\section{Discussion}

It has been proposed that microvascular dysfunction plays an important role in CSX, but direct evidence is lacking. In the current study, sublingual microscopy demonstrated that patients with CSX can be characterized by glycocalyx-mediated microvascular dysfunction in comparison with volunteer control subjects. The CSX patients exhibit a larger PBR at baseline, reflecting deeper penetration of circulating erythrocytes towards the endothelium, indicating a decrease in glycocalyx integrity. Additionally, the physiological increase in PBR upon nitroglycerin challenge that was observed in the volunteers was absent in patients with CSX. The patients with obstructive CAD had a similar large median PBR at baseline as the CSX patients and also failed to show a significant increase upon nitroglycerin challenge. However, it should be noted that a considerably wider variation in PBR's was observed in the CAD patients. This may indicate that there is heterogeneity in the contribution of microvascular disease in patients with obstructive $C A D$, and possibly even absence of microvascular dysfunction in a proportion of these patients. 
Adenosine stress CMR perfusion imaging in our CSX patients showed no evidence of visual perfusion defects, but revealed that the perfusion reserve was significantly lower in the subendocardium as compared with the subepicardium. The strong correlation that was found between the sublingual PBR-response and the CMR-derived MPRI measurements in CSX patients supports the concept that the glycocalyx plays an important role in the regulation of microvascular volume for myocardial perfusion and indicates a glycocalyx loss in CSX patients. ${ }^{25}$ Sublingual PBR measurements may therefore be a useful tool for non-invasively characterizing microvascular dysfunction in CSX.

\section{Sublingual microvasculature imaging}

A major problem in the assessment of coronary microvascular dysfunction and its role in occurrence of myocardial ischemia in CSX is that the direct evaluation of the structure and function of small coronary vessels is cumbersome. ${ }^{6}$ Intravital microscopy has emerged as a promising non-invasive tool to assess the microcirculation directly in easily accessible regions. To our knowledge, this is the first study to investigate the glycocalyx-mediated microvascular responsiveness in patients with CSX and obstructive CAD. Our results are in line with previous clinical studies that have reported evidence of endothelial glycocalyx loss in patients with accelerated vascular disease (e.g. patients with type 1 diabetes, ${ }^{12}$ dialysis patients, ${ }^{14}$ and lacunar stroke patients with white matter lesions. ${ }^{15}$ The underlying mechanisms for endothelial glycocalyx perturbation during these conditions of increased cardiovascular risk are currently not fully understood. ${ }^{17}$ Since the endothelial glycocalyx is anticipated to act as a protective layer which shields the vessel wall from atherogenic stimuli, impairment of this protective barrier is considered a primary step in microvascular dysfunction. ${ }^{17}$ Moreover, it has been demonstrated in animal studies that an intact endothelial glycocalyx exhibits an increase in bloodperfused microvascular volume after nitroglycerin or adenosine challenge by increasing glycocalyx accessibility. ${ }^{19,25}$ These findings imply that the glycocalyx also plays an important role in the regulation of microvascular volume for perfusion. ${ }^{19,25}$ The strong correlation between the sublingual PBR-response and the CMR-derived MPRI measurements seems to support this role, and indicates a generalized glycocalyx loss in patients with CSX.

\section{CMR perfusion imaging}

Over the last decade, several studies have explored the ability of CMR perfusion imaging to detect myocardial ischemia in patients with $\mathrm{CSX}^{8-11}$ However, these studies differed considerably in study design and reported conflicting results. ${ }^{8-11}$ Panting et al. used adenosine stress perfusion CMR at 1.5 Tesla and reported evidence of hypoperfusion in the subendocardium in patients with CSX. ${ }^{8}$ In an attempt to reproduce these data Vermeltfoort et al., however, found that CSX patients have a similar increase in 
myocardial perfusion during adenosine in both the subendocardium and subepicardium. ${ }^{9}$ The fact that Panting et al. included only patients with ST-segment depression on exercise electrocardiography whereas Vermeltfoort et al. also included patients with an abnormal perfusion single-photon emission computed tomography (and only $25 \%$ with ST-depression), suggests that the study populations of these 2 studies are not directly comparable. A recent study by Karamitsos et al. investigated 18 CSX patients, all demonstrating ST-depression on exercise electrocardiography, using CMR imaging for absolute quantification of the myocardial blood flow at 3.0 Tesla. ${ }^{11}$ Although they found no evidence of transmural hypoperfusion in these CSX patients, no comparison was made between subendocardial and subepicardial myocardial blood flow. As a result, subendocardial hypoperfusion may not have been detected.

In the current study, the MPI increased significantly during adenosine stress in both subendocardial and subepicardial segments. However, we observed that the MPI reserve of the subendocardium (i.e. the subendocardial MPRI) was significantly lower as compared with the subepicardial MPRI. While glycocalyx-mediated microvascular dysfunction is expected to affect the myocardium diffusely, the subendocardium seems also in other forms of microvascular disease (e.g. cardiac transplant arteriopathy and systemic inflammation) to be more vulnerable to hypoperfusion than the subepicardium. ${ }^{26-28}$ This subendocardial vulnerability could be due to a slightly higher metabolic demand and a lower blood supply. ${ }^{29-31}$ In addition, Algranati et al. found that this subendocardial vulnerability to hypoperfusion stems from the combined effects of cardiac contraction, vascular pressure-dependent compliance, and potential transmural differences in vessel anatomy. ${ }^{31}$ Thus, diffuse microvascular glycocalyx perturbation which also can be considered an alteration of the vessel wall properties may therefore result in perfusion disturbances most prominent in subendocardial layers.

Although a difference in subendocardial and subepicardial MPRI is to some extent the result of normal physiological mechanisms, it has been suggested by Panting et al. that the more pronounced difference in this subendocardial-to-subepicardial MPRI ratio in CSX patients can be used to distinguish between CSX patients and healthy controls. ${ }^{8,32}$ We used a higher magnetic field strength and imaging sequence and it should be noted that the homogenous group of CSX patients investigated in the current study demonstrated a considerable range of subendocardial-to-subepicardial MPRI's.

\section{Limitations}

The characteristics of patients included in previous CSX studies are highly variable. ${ }^{4}$ The current study therefore used a strict definition of CSX aimed at including a homogeneous group of patients, albeit with a modest sample size. ${ }^{4}$ Controversy remains on the inclusion of CSX patients with cardiovascular risk factors (e.g. hypertension or hypercholesterolemia) as they may form underlying causes of microvascular dysfunction. ${ }^{6,11}$ In accordance with the current recommendations and guidelines, we did not exclude CSX 
patients or control subjects that had a history of (medically) controlled mild hypertension or hypercholesterolemia. As a result, our study groups showed similar prevalence of these cardiovascular risk factors, implying that the observed differences in the glycocalyx-mediated microvascular responsiveness between the 3 study groups cannot be attributed to these risk factors.

\section{Conclusions and future perspectives}

The prognostic consequences of CSX are not well described and the treatment of patients with CSX currently consists predominantly of risk factor modification and symptom control. However, CSX patients frequently have persisting or even worsening symptoms over time, are often referred to further and repeated (non-)invasive testing and the quality of life is impaired. ${ }^{1}$ Although novel drugs may offer beneficial effects, the optimal therapeutic strategy in CSX patients currently remains unknown. ${ }^{6,33}$ The results of the current study show that sublingual microscopy is a promising diagnostic tool to characterize CSX patients by glycocalyx-mediated microvascular dysfunction. Since sublingual PBR-responsiveness correlated strongly to the CMR-derived myocardial perfusion reserve, the glycocalyx may play an important role in the regulation of microvascular volume for myocardial perfusion. Therefore, CSX patients might benefit from therapeutic interventions aimed at restoring the endothelial function by limiting the degradation of the endothelial glycocalyx as well as supplementation of a damaged glycocalyx. ${ }^{34,35}$

\section{References}

1. Crea F, Lanza GA. Angina pectoris and normal coronary arteries: cardiac syndrome X. Heart 2004;90:45763.

2. Gulati M, Cooper-DeHoff RM, McClure C, et al. Adverse cardiovascular outcomes in women with nonobstructive coronary artery disease: a report from the Women's Ischemia Syndrome Evaluation Study and the St James Women Take Heart Project. Arch Intern Med 2009;169:843-50.

3. Jespersen L, Hvelplund A, Abildstrom SZ, et al. Stable angina pectoris with no obstructive coronary artery disease is associated with increased risks of major adverse cardiovascular events. Eur Heart J 2012;33:734-44.

4. Fox K, Garcia MA, Ardissino D, et al. Guidelines on the management of stable angina pectoris: executive summary: The Task Force on the Management of Stable Angina Pectoris of the European Society of Cardiology. Eur Heart J 2006;27:1341-81.

5. Cannon RO, 3rd, Epstein SE. "Microvascular angina" as a cause of chest pain with angiographically normal coronary arteries. Am J Cardiol 1988;61:1338-43.

6. Lanza GA. Cardiac syndrome X: a critical overview and future perspectives. Heart 2007;93:159-66.

7. Lockie $T$, Ishida $M$, Perera $D$, et al. High-resolution magnetic resonance myocardial perfusion imaging at 3.0-Tesla to detect hemodynamically significant coronary stenoses as determined by fractional flow reserve. J Am Coll Cardiol 2011;57:70-5. 
8. Panting JR, Gatehouse PD, Yang GZ, et al. Abnormal subendocardial perfusion in cardiac syndrome $X$ detected by cardiovascular magnetic resonance imaging. N Engl J Med 2002;346:1948-53.

9. Vermeltfoort IA, Bondarenko O, Raijmakers PG, et al. Is subendocardial ischaemia present in patients with chest pain and normal coronary angiograms? A cardiovascular MR study. Eur Heart J 2007;28:1554-8.

10. Lanza GA, Buffon A, Sestito A, et al. Relation between stress-induced myocardial perfusion defects on cardiovascular magnetic resonance and coronary microvascular dysfunction in patients with cardiac syndrome X. J Am Coll Cardiol 2008;51:466-72.

11. Karamitsos TD, Arnold JR, Pegg TJ, et al. Patients with syndrome $X$ have normal transmural myocardial perfusion and oxygenation: a 3-T cardiovascular magnetic resonance imaging study. Circ Cardiovasc Imaging 2012;5:194-200.

12. Nieuwdorp M, Mooij HL, Kroon J, et al. Endothelial glycocalyx damage coincides with microalbuminuria in type 1 diabetes. Diabetes 2006;55:1127-32.

13. Broekhuizen LN, Lemkes BA, Mooij HL, et al. Effect of sulodexide on endothelial glycocalyx and vascular permeability in patients with type 2 diabetes mellitus. Diabetologia 2010;53:2646-55.

14. Vlahu CA, Lemkes BA, Struijk DG, Koopman MG, Krediet RT, Vink H. Damage of the endothelial glycocalyx in dialysis patients. J Am Soc Nephrol 2012;23:1900-8.

15. Martens RJ, Vink H, van Oostenbrugge RJ, Staals J. Sublingual Microvascular Glycocalyx Dimensions in Lacunar Stroke Patients. Cerebrovasc Dis 2013;35:451-454.

16. Mulders TA, Nieuwdorp M, Stroes ES, Vink H, Pinto-Sietsma SJ. Non-invasive assessment of microvascular dysfunction in families with premature coronary artery disease. Int J Cardiol 2013;168:5026-8.

17. Van Teeffelen JW, Brands J, Stroes ES, Vink H. Endothelial glycocalyx: sweet shield of blood vessels. Trends Cardiovasc Med 2007;17:101-5.

18. Nieuwdorp M, van Haeften TW, Gouverneur MC, et al. Loss of endothelial glycocalyx during acute hyperglycemia coincides with endothelial dysfunction and coagulation activation in vivo. Diabetes 2006;55:480-6.

19. VanTeeffelen JW, Constantinescu AA, Brands J, Spaan JA, Vink H. Bradykinin- and sodium nitroprussideinduced increases in capillary tube haematocrit in mouse cremaster muscle are associated with impaired glycocalyx barrier properties. J Physiol 2008;586:3207-18

20. Plein S, Ryf S, Schwitter J, Radjenovic A, Boesiger P, Kozerke S. Dynamic contrast-enhanced myocardial perfusion MRI accelerated with k-t sense. Magn Reson Med 2007;58:777-85.

21. Cerqueira MD, Weissman NJ, Dilsizian V, et al. Standardized myocardial segmentation and nomenclature for tomographic imaging of the heart: a statement for healthcare professionals from the Cardiac Imaging Committee of the Council on Clinical Cardiology of the American Heart Association. Circulation 2002;105:539-42.

22. Patlak CS, Blasberg RG, Fenstermacher JD. Graphical evaluation of blood-to-brain transfer constants from multiple-time uptake data. J Cereb Blood Flow Metab 1983;3:1-7.

23. Kurita T, Sakuma H, Onishi K, et al. Regional myocardial perfusion reserve determined using myocardial perfusion magnetic resonance imaging showed a direct correlation with coronary flow velocity reserve by Doppler flow wire. Eur Heart J 2009;30:444-52.

24. Ichihara T, Ishida M, Kitagawa K, et al. Quantitative analysis of first-pass contrast-enhanced myocardial perfusion MRI using a Patlak plot method and blood saturation correction. Magn Reson Med 2009;62:373-83.

25. Brands J, Spaan JA, Van den Berg BM, Vink H, VanTeeffelen JW. Acute attenuation of glycocalyx barrier properties increases coronary blood volume independently of coronary flow reserve. Am J Physiol Heart Circ Physiol 2010;298:H515-23.

26. Kobayashi Y, Giles JT, Hirano M, et al. Assessment of myocardial abnormalities in rheumatoid arthritis using a comprehensive cardiac magnetic resonance approach: a pilot study. Arthritis Res Ther 2010;12:R171.

27. Muehling OM, Wilke NM, Panse $P$, et al. Reduced myocardial perfusion reserve and transmural perfusion gradient in heart transplant arteriopathy assessed by magnetic resonance imaging. J Am Coll Cardiol 2003;42:1054-60. 
28. Ishimori ML, Martin R, Berman DS, et al. Myocardial ischemia in the absence of obstructive coronary artery disease in systemic lupus erythematosus. JACC Cardiovasc Imaging 2011;4:27-33.

29. Hoffman JI, Baer RW, Hanley FL, Messina LM. Regulation of transmural myocardial blood flow. J Biomech Eng 1985;107:2-9.

30. Hoffman JI. Transmural myocardial perfusion. Prog Cardiovasc Dis 1987;29:429-64.

31. Algranati D, Kassab GS, Lanir Y. Why is the subendocardium more vulnerable to ischemia? A new paradigm. Am J Physiol Heart Circ Physiol 2010;300:H1090-100.

32. Larghat A, Biglands J, Maredia N, et al. Endocardial and epicardial myocardial perfusion determined by semi-quantitative and quantitative myocardial perfusion magnetic resonance. Int J Cardiovasc Imaging 2012;28:1499-511.

33. Mehta PK, Goykhman P, Thomson LE, et al. Ranolazine improves angina in women with evidence of myocardial ischemia but no obstructive coronary artery disease. JACC Cardiovasc Imaging 2011;4:514-22.

34. VanTeeffelen JW, Brands J, Vink H. Agonist-induced impairment of glycocalyx exclusion properties: contribution to coronary effects of adenosine. Cardiovasc Res 2010;87:311-9.

35. Becker BF, Chappell D, Bruegger D, Annecke T, Jacob M. Therapeutic strategies targeting the endothelial glycocalyx: acute deficits, but great potential. Cardiovasc Res 2010;87:300-10.

36. Nieuwdorp M, Meuwese MC, Vink H, Hoekstra JB, Kastelein JJ, Stroes ES. The endothelial glycocalyx: a potential barrier between health and vascular disease. Curr Opin Lipidol 2005;16:507-11.

37. Goedhart PT, Khalilzada M, Bezemer R, Merza J, Ince C. Sidestream Dark Field (SDF) imaging: a novel stroboscopic LED ring-based imaging modality for clinical assessment of the microcirculation. Opt Express 2007;15:15101-14. 


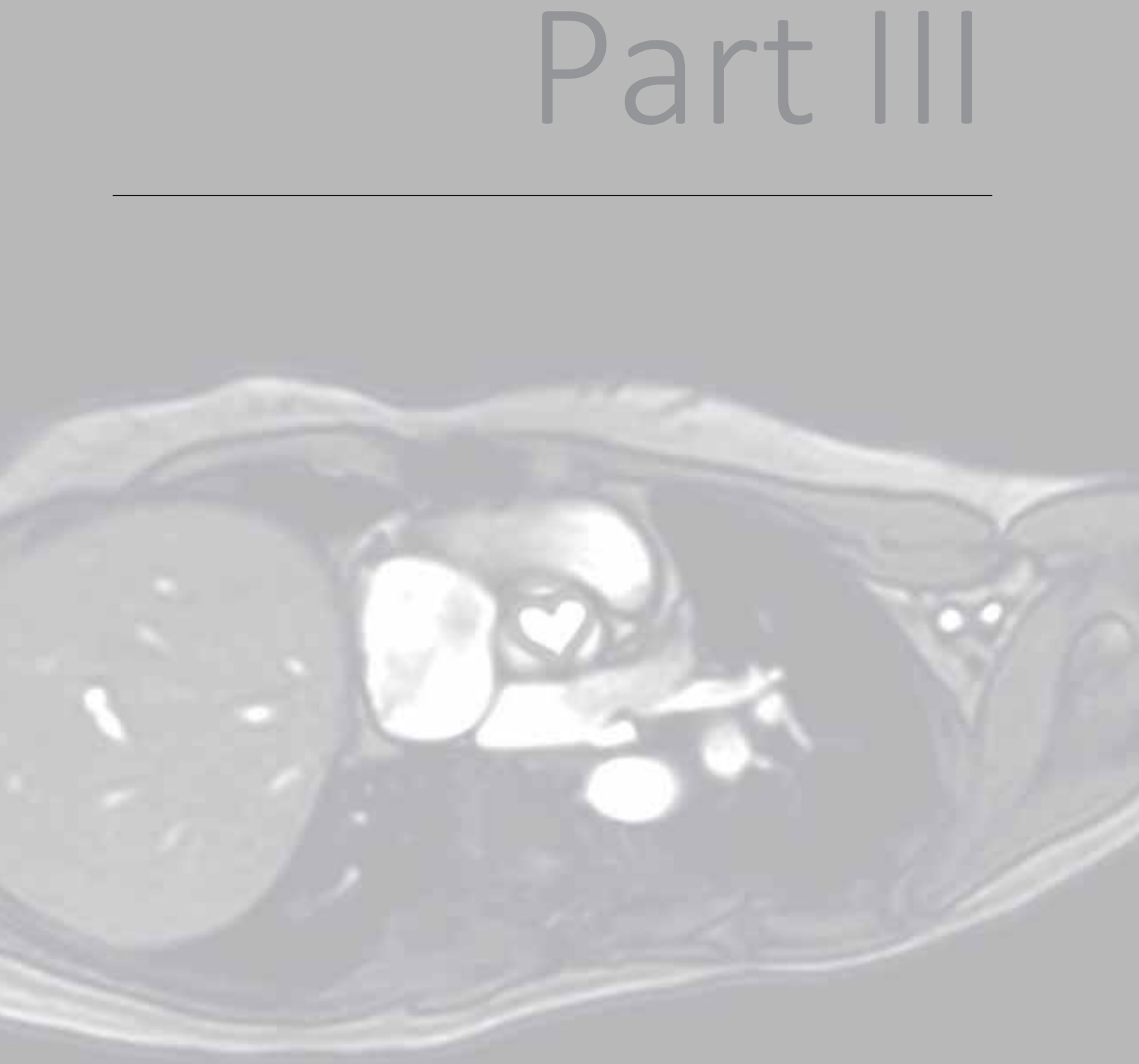



\section{Replacement and reactive myocardial}

\section{fibrosis in idiopathic dilated}

\section{cardiomyopathy:}

Comparison of magnetic resonance imaging with right ventricular biopsy

S. Schalla, S.C. Bekkers, R. Dennert, R.J. van Suylen, J. Waltenberger, T. Leiner, J. Wildberger, H.J. Crijns, S. Heymans.

Eur J Heart Fail. 2010 Mar;12(3):227-31. 


\section{Abstract}

Background: Focal (replacement) myocardial fibrosis detected with late gadolinium enhancement (LGE) magnetic resonance imaging (CMR) and interstitial (reactive) fibrosis measured on endomyocardial biopsy specimens are associated with an adverse prognosis in patients with idiopathic dilated cardiomyopathy (DCM). We described the patterns of focal fibrosis in a group of consecutive patients referred to our hospital with DCM and tested the hypothesis whether focal and interstitial fibrosis are linked to each other or to myocardial inflammation.

Methods: Fifty-six patients with DCM underwent CMR and right ventricular (RV) biopsy to determine focal fibrosis, RV and left ventricular (LV) volumes, ejection fraction, mass as well as interstitial fibrosis, inflammatory cells and viral load.

Results: Twenty-two patients (39\%) showed LGE of the LV myocardium (mean extent $4 \pm 8 \%$ ), none of the RV: Eight patients showed midmyocardial, 3 mid- to epicardial, 5 transmural and 6 more than one LGE pattern. No correlation was found between the presence $(r=0.125, p=0.893, A \cup C=0.51,95 \% \mathrm{Cl} 0.35-0.67)$ or amount $(r<0.01)$ of LGE and interstitial fibrosis. The myocardial viral load was not correlated with focal or interstitial fibrosis. No correlation was found between interstitial fibrosis and inflammatory cells. The distributions of CD3 ( $p=0.003)$ and CD4 ( $p=0.02)$ positive cells were significantly different between patients with and without focal fibrosis.

Conclusions: Focal LV myocardial fibrosis was not correlated to the amount of interstitial fibrosis and both forms were not related to the degree of LV or RV dilation and impairment of systolic function. Focal fibrosis might be related to inflammation rather than interstitial fibrosis. 


\section{Introduction}

The presence of focal myocardial fibrosis, also called scar or replacement fibrosis, detected with late Gadolinium enhancement (LGE) cardiovascular magnetic resonance imaging (CMR) is a predictor of adverse outcome in patients with idiopathic dilated cardiomyopathy (DCM). ${ }^{1,2}$ Increased interstitial (reactive) fibrosis determined with histologic staining from endomyocardial biopsy (EMB) specimens has been described in DCM and associated with an adverse prognosis and impaired response to therapeutic interventions in selected groups of patients with DCM. ${ }^{3-6}$

We sought to describe the patterns of focal fibrosis in a group of patients with DCM and correlate focal fibrosis with interstitial fibrosis, myocardial viral load and inflammation. A pilot study in 10 autopsies was performed to compare interstitial fibrosis from right ventricular (RV) with left ventricular (LV) EMB.

\section{Methods}

\section{Study population}

Study subjects were 56 consecutive patients referred to our hospital with globally impaired left ventricular (LV) systolic function determined with echocardiography (LV ejection fraction (EF) $<45 \%$ ). Patients with a history of myocardial infarction, hypertension, valvular heart disease or other known causes for impaired systolic function such as a history of cardiotoxic agents were not enrolled in the study. CMR, endomyocardial biopsy and, to rule out significant coronary artery disease as the underlying cause of impaired systolic function, coronary angiography $(n=53)$ or computed tomography angiography $(n=3)$ were also performed. Patients with infarct-like subendocardial or transmural late enhancement patterns within the territory of a coronary artery and corresponding regional wall motion abnormalities only were not included to avoid the inclusion of patients with an embolic event. The study complied with the Declaration of Helsinki and was approved by the local ethics committee.

\section{Endomyocardial biopsy}

Right ventricular (RV) endomyocardial biopsies were obtained using a transcatheter bioptome (Cordis, USA). Three biopsy specimens per patients were processed and analyzed to determine the collagen volume fraction. After fixation in formalin and embedding in paraffin, $3 \mu \mathrm{m}$ thick histological sections were cut and stained with Sirius red. The total tissue area per histological slide was determined by morphometrical analysis (morphometrical software: Leica Qwin, version 3, Cambridge, UK). The amount of collagen (collagen volume fraction, CVF) was quantified as percentage Sirius red stained area 
per total myocardial tissue area, excluding perivascular fibrosis. ${ }^{7-9}$ The analysis was performed by one experienced investigator (R.S.) blinded to patient details.

In addition, inflammatory cells were quantified. Tissue sections were subjected to immunohistochemical stainings using CD3, CD4, CD8, CD20, CD45, and CD68 antibodies (DAKO, Denmark). The numbers of stained inflammatory cells were counted and expressed per square millimetre.

DNA to detect Parvovirus B19, Human Herpes virus 6 and Epstein-Barr virus was extracted from 2 pooled endomyocardial biopsies using a QIAamp DNA blood mini kit (Qiagen, Venlo, The Netherlands). DNA concentrations were determined using a nanodrop instrument (Thermo Fischer Scientific, Wilmington, DE, USA). Before extraction, all samples were spiked with murine CMV DNA or RNA, which was used as an extraction and amplification control. Reverse transcription was performed using Taqman Reverse Transcriptase reagents (Applied Biosystems, Foster City, CA, USA). The PCR mix consisted of $20 \mu \mathrm{l}$ isolated DNA, final concentrations of $600 \mathrm{nM}$ of each primer and 200nM of the probe and $1 \times$ Absolute QPCR mix (Abgene, Epsom, UK). All probes were labeled with FAM as a reporter dye and TAMRA as a quencher dye. All real-time PCR reactions were performed using a ABI prism 7000 (Applied Biosystems, Foster City, CA, USA) and quantified using a standard curve. The quality of the assays was assured by positive and negative controls as well as a test on amplification inhibition in each sample by an external amplification control. For quantification of viral loads, standard curves were included in each run.

\section{Magnetic resonance imaging}

Patients were examined with a 1.5 Tesla MR scanner (Gyroscan Intera, Philips Medical Systems, Best, the Netherlands). ECG-gated cine images were acquired for functional analysis during multiple breath holds using a steady-state free precession sequence (slice thickness $6 \mathrm{~mm}$, slice gap $4 \mathrm{~mm}$, TR/TE 3.8/1.9 ms, flip angle 50', FOV $350 \mathrm{~mm}$, matrix $256 \times 256,22-25$ phases per cardiac cycle) in 2-, 3- and 4-chamber long axis and contiguous short axis views. For the detection of myocardial edema or acute inflammation, multislice short axis images were acquired using a dual-inversion black-blood T2weighted sequence (T2 SPIR, slice thickness $8 \mathrm{~mm}$, slice gap $2 \mathrm{~mm}$, TR/TE 1600/100 ms, flip angle $90^{\circ}$, FOV $350 \mathrm{~mm}$, matrix $512 \times 512$ ) with fat suppression. A breath hold multislice T1-weighted 3D inversion-recovery gradient-echo sequence (slice thickness 12 $\mathrm{mm}$, slice gap $-6 \mathrm{~mm}$, TR/TE $4.2 / 1.3 \mathrm{~ms}$, flip angle $15^{\circ}$, FOV $400 \mathrm{~mm}$, resolution $256 \mathrm{x}$ $256)$ to evaluate the presence of focal myocardial fibrosis was used to acquire images in 2-, 4-chamber long axis and short axis orientation 10 minutes after intravenous administration of $0.2 \mathrm{mmol} / \mathrm{kg}$ Gadolinium-DTPA (Magnevist, Schering AG, Germany). Inversion times were adjusted to null normal myocardium (200-300ms).

MR images were analyzed with the CAAS MRV 3.0 software (Pie Medical Imaging, Maastricht, the Netherlands). Endocardial and epicardial LV and endocardial RV contours 
were manually traced in end-diastolic and end-systolic phases on short axis slices to determine LV and RV end-diastolic volume (EDV), end-systolic volume (ESV), ejection fraction (EF) and LV end-diastolic mass. Areas of late enhancement were visually assessed by 2 independent readers blinded to patient details. If present, areas of fibrosis were quantified by planimetry using a signal cut-off of $>2$ SD above the mean signal intensity of remote myocardium in the same slice and expressed as a percentage of total LV mass.

\section{Statistical analysis}

Data are expressed as median values with interquartile ranges. Differences in LV and RV EDV, EF and LV mass, CVF, viral load and amount of inflammatory cells between patients with or without focal fibrosis as determined by LGE CMR were calculated by means of the Mann-Whitney test and differences in age by Student's $t$ test. ReceiverOperating Characteristic analysis, with corresponding measures of statistical uncertainty (95\% confidence intervals, CI), was applied to the LGE and CVF values used to detect interstitial fibrosis. Correlations were calculated by means of the Spearman's rank correlation method.

\section{Pilot study}

A pilot study to compare interstitial fibrosis from RV with LV endomyocardial biopsy was performed in 10 consecutive autopsies. Biopsy specimens were taken from both the RV septal wall in accordance with the location of standard biopsies from transcathether sampling and from the LV anterior wall. Patients with histological signs of fibrosis due to prior myocardial infarction or severe hypertrophy were not included in the pilot study. A good correlation between LV (3.2\%, 1.7-5.9) and RV interstitial fibrosis (2.2\%, 1.7-5.7) was found $(r=0.8, p=0.5)$.

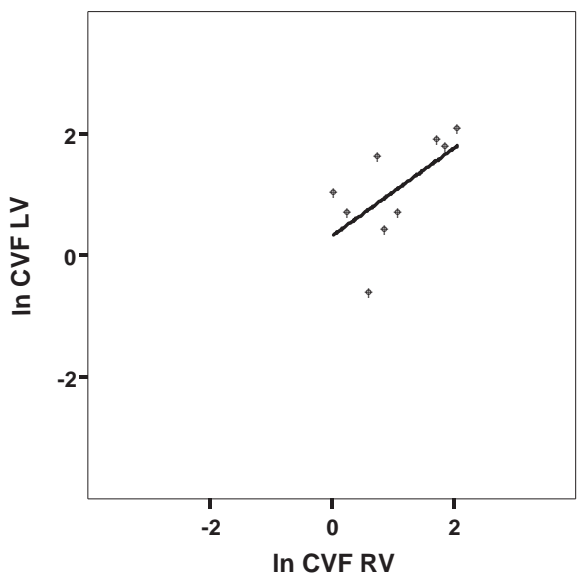

Figure 1: Pilot study comparing interstitial fibrosis determined with histologic staining (logarithmic values (In) for collagen volume fraction (CVF), \%) from postmortem right (RV) and left ventricular (LV) endomyocardial biopsy specimens. A good correlation was found between RV and LV biopsy samples $(r=0.8)$. 


\section{Results}

Endomyocardial biopsy and CMR were performed in 30 male and 26 female patients. Patient characteristics are shown in table 1. Twenty-five patients (45\%) were in NYHA functional class I, 19 (34\%) in class II, 12 (21\%) in class III and none in class IV.

In 2 patients the systolic function had improved under medical therapy and became normal (ejection fraction $>55 \%$ ) at the time of CMR, while initial echocardiography at the referring hospital clearly showed impaired systolic function (LVEF 44 and 20\%). Eighteen patients (32\%) had an increased LV mass. ${ }^{10}$ No patient had signs of edema on T2-weighted images. There were no patients with pericardial effusion.

Focal LV fibrosis on LGE CMR was observed in 22 patients (39\%; median extent 5\%, 314\%). Eight patients showed midmyocardial, 3 mid- to epicardial, 5 mid- to transmural and 6 more than one LGE pattern (figure 2 and 3). No patient had focal RV fibrosis. The presence or extent of focal LV fibrosis as measured with LGE CMR did not correlate with age, LV EDV, LV mass, LV EF, RV EDV or RV EF. Thus, the severity of LV dilation or impairment of systolic function was not related to the presence or the amount of focal fibrosis.

Table 1: Characteristics of the 56 patients with idiopathic dilated cardiomyopathy.

\begin{tabular}{|c|c|c|c|c|}
\hline & \multirow[b]{2}{*}{$\begin{array}{l}\text { All Patients } \\
(n=56)\end{array}$} & \multicolumn{3}{|l|}{ LGE Status } \\
\hline & & $\begin{array}{l}+ \text { LGE } \\
(n=22)\end{array}$ & $\begin{array}{l}- \text { LGE } \\
(n=34)\end{array}$ & $\mathrm{p}$ Value \\
\hline Age (years) & $48(36-60)$ & $50(33-64)$ & $48(37-62)$ & 0.997 \\
\hline Male gender, $\mathrm{n}$ & 30 & 14 & 16 & \\
\hline LV EDV (ml) & $216(170-287)$ & $237(187-300)$ & $207(156-260)$ & 0.161 \\
\hline LV EDV / BSA $\left(\mathrm{ml} / \mathrm{m}^{2}\right)$ & $112(92-145)$ & $125(102-158)$ & $107(90-130)$ & 0.135 \\
\hline LV EF (\%) & $36(24-45)$ & $30(22-46)$ & $37(27-53)$ & 0.450 \\
\hline LV mass (g) & $128(102-169)$ & $137(103-183)$ & $120(97-157)$ & 0.115 \\
\hline LV mass / BSA $\left(\mathrm{g} / \mathrm{m}^{2}\right)$ & $70(55-79)$ & $73(58-81)$ & $69(53-90)$ & 0.196 \\
\hline RV EDV & $156(130-178)$ & $152(120-177)$ & $156(132-179)$ & 0.563 \\
\hline $\mathrm{RV}$ EDV / BSA $\left(\mathrm{ml} / \mathrm{m}^{2}\right)$ & $79(68-90)$ & $77(61-88)$ & $81(70-103)$ & 0.314 \\
\hline RV EF (\%) & $49(40-55)$ & $48(39-57)$ & $49(40-54)$ & 0.620 \\
\hline Collagen volume fraction (\%) & $4.9(2.1-6.4)$ & $4.9(1.9-6.9)$ & $4.8(2.6-6.4)$ & 0.893 \\
\hline
\end{tabular}

The $\mathrm{p}$ value represents the comparison of patients with LGE versus those without LGE detected with magnetic resonance imaging (Mann Whitney test). Data are expressed as median (interquartile range).

LGE denotes late Gadolinium enhancement, LV EDV left ventricular end-diastolic volume, LV EF left ventricular ejection fraction, LV mass left ventricular mass, RV EDV right ventricular end-diastolic volume, RV EF right ventricular ejection fraction measured with MR imaging; collagen volume fraction is derived from histologic staining. BSA denotes body surface area. 

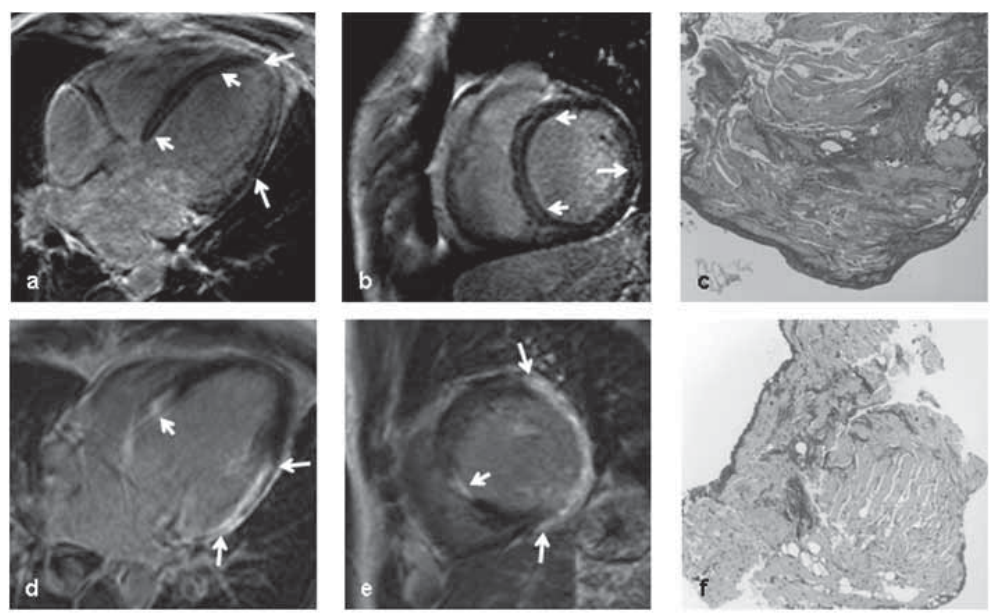

Figure 2: Late gadolinium enhancement MR images ( $a, b, d, e)$ of 2 patients with large areas of fibrosis and corresponding histology (c, f).

Top: A patient with fibrosis in a midmyocardial pattern visible as midmyocardial enhancement of septal and lateral wall on 4-chamber (a) and short axis view (b); histology also shows increased interstitial fibrosis (collagen volume fraction 7.1\%).

Bottom: A patient with transmural fibrosis pattern visible as transmural enhancement of the septal, inferior, inferolateral and lateral wall without evidence of coronary artery disease. The collagen volume fraction was $4.6 \%$.
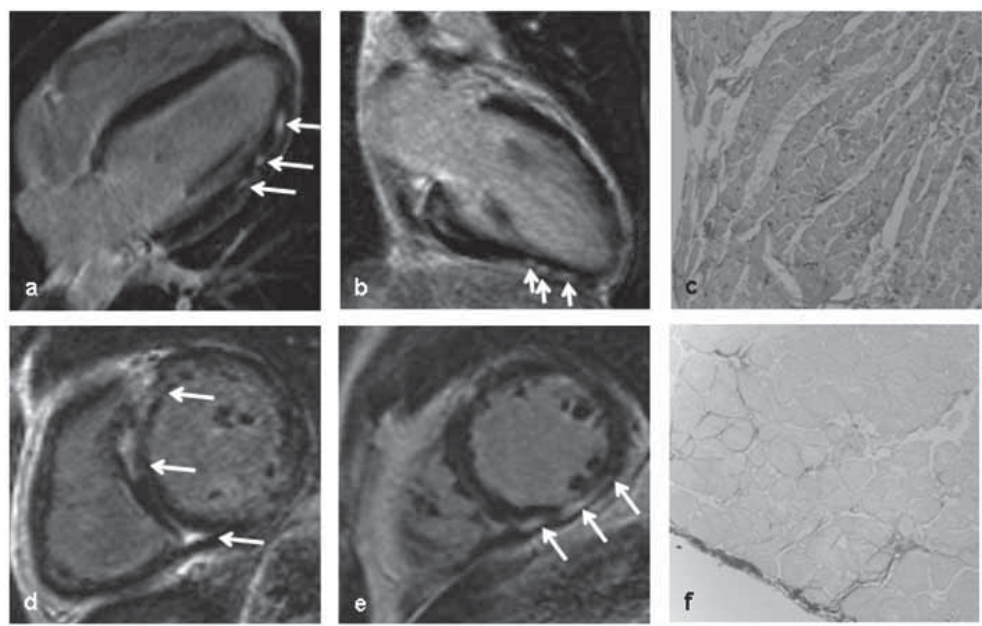

Figure 3: Late gadolinium enhancement MR images ( $a, b, d, e)$ of 2 patients with smaller and more localized areas of fibrosis and corresponding histology (c, f).

Top: A patient with localized fibrosis in a midmyocardial to epicardial pattern visible as 3 regions lateral on 4-chamber (a) and inferior on 2-chamber view (b); the collagen volume fraction was $2 \%$.

Bottom: A patient with patchy midmyocardial fibrosis on short axis views (d, e). The collagen volume fraction was $1.7 \%$. 
The median CVF was 4.9\% (2.1-6.4\%). Older patients did not show more interstitial fibrosis than younger patients. The CVF was neither correlated with LV EDV, EF or mass nor with RV EDV or EF. No differences were found between patients with or without focal fibrosis detected with LGE and the amount of CVF (figure 4 and 5): the presence of focal fibrosis was not related to the amount of interstitial fibrosis (area under the curve $=0.51,95 \% \mathrm{Cl} 0.35-0.67)$. The amount of focal fibrosis measured with LGE as \% of LV was also not correlated with CVF.

Myocardial viral load was not correlated with focal or interstitial fibrosis. No correlation was found between interstitial fibrosis and inflammatory cells. The numbers of CD3 ( $p=0.003)$ and CD4 ( $p=0.02)$ positive inflammatory cells were significantly differently distributed between patients with and without focal fibrosis

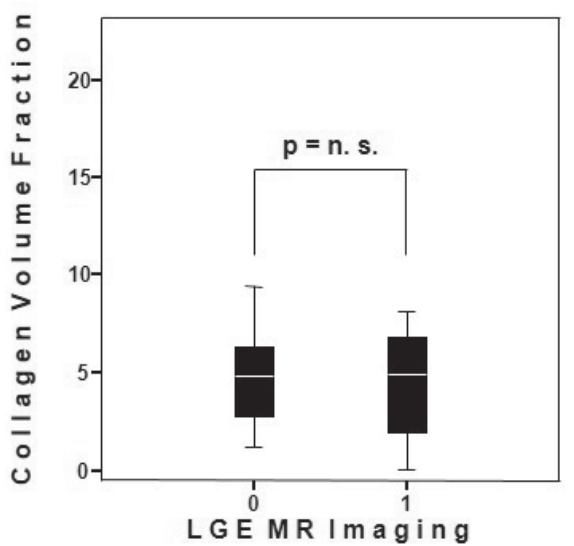

Figure 4a-c: a, top) Box plot of a) interstitial fibrosis determined with histologic staining (collagen volume fraction, \%), b, middle) CD3 positive cells (cells $/ \mathrm{mm}^{2}$ ) and $\mathrm{c}$, bottom) CD4 positive cells (cells $/ \mathrm{mm}^{2}$ ) versus focal fibrosis as detected with late gadolinium enhancement MR imaging (LGE MR Imaging): 0 denotes no enhancement, 1 denotes the presence of areas of focal enhancement, n.s. denotes not significant. The distributions of CD3 and CD4 positive inflammatory cells were significantly different between patients with and without focal fibrosis on MR images. The amount of interstitial fibrosis was not different between patients with and without focal fibrosis.
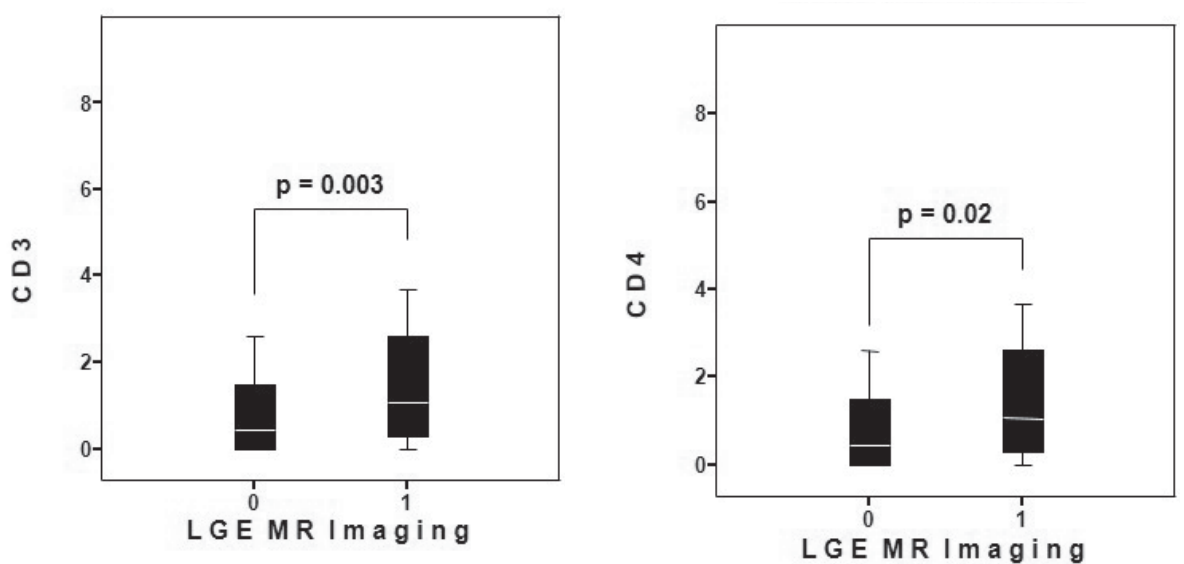


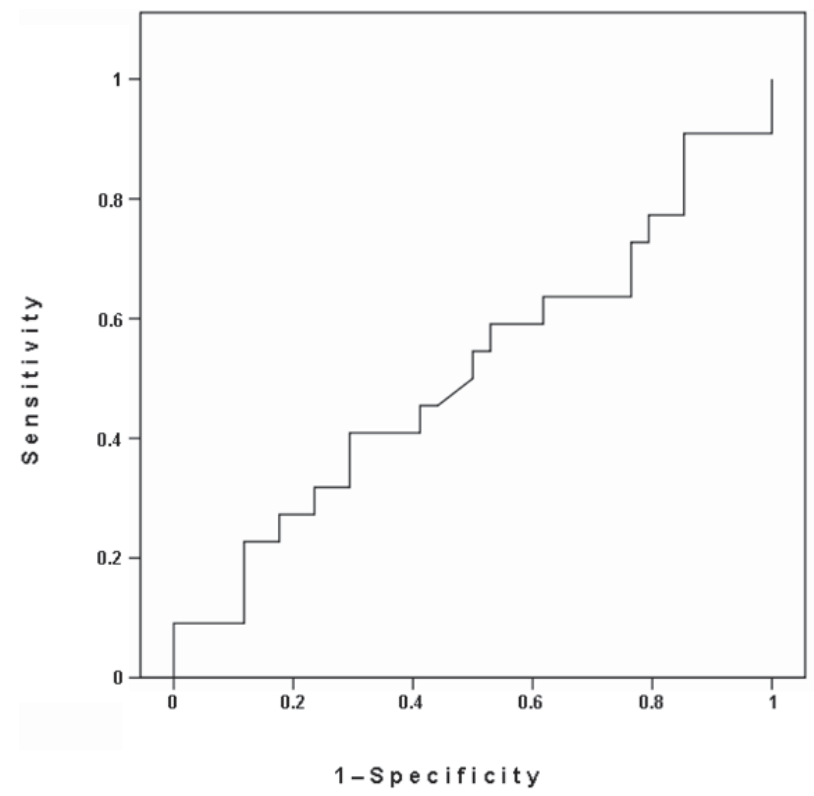

Figure 5: ROC curve to assess the ability of late gadolinium enhancement MR imaging (presence of focal fibrosis) to predict interstitial fibrosis as determined with histologic staining. The area under the curve is $0.51(95 \% \mathrm{Cl} 0.35$ 0.67). The presence of focal fibrosis is not linked to a high amount of interstitial fibrosis.

\section{Discussion}

The main findings of the study are that 1 ) focal (replacement) fibrosis as detected with LGE CMR was not correlated to the amount of interstitial (reactive) fibrosis in patients with DCM, and, 2) the severity of LV or RV dilation and systolic dysfunction were not related to focal or interstitial fibrosis. The myocardial viral load was not correlated with focal or interstitial fibrosis. However, the distributions of CD3 and CD4 positive inflammatory cells were significantly different between patients with and without focal fibrosis on MR images. Thus, focal fibrosis might be related to inflammation rather than interstitial fibrosis.

CMR has an established role in the detection of focal fibrosis, and the presence of focal fibrosis is associated with an adverse prognosis. ${ }^{1,}{ }^{11}$ LGE was present in $39 \%$ of patients in our study group and, thus, comparable to other results. ${ }^{1,2}$ Bello et al. found LGE only in $12 \%$ of patients with non-ischemic systolic LV-dysfunction. ${ }^{12}$ McCrohon et al. observed in $13 \%$ of patients with systolic LV dysfunction LGE in an ischemic-type pattern despite normal coronary angiographies, $28 \%$ had patchy or linear mid-epicardial LGE. ${ }^{11}$ 
LGE imaging has sometimes been considered the method of choice to detect myocardial fibrosis. ${ }^{13}$ However, it is a method to delineate focal fibrosis, and other MR imaging techniques have been applied for the detection of interstitial fibrosis at an experimental stage with time consuming image analysis. ${ }^{14-17}$ Thus, for the assessment of interstitial fibrosis, MR imaging is currently not considered a routine method and only preliminary and contradictory results have been published. ${ }^{14,15,17}$ An in-vitro study in normal myocardium, myocardium with increased interstitial fibrosis and myocardium with infarction, compared histological staining with 3 Tesla spin echo imaging (to determine Gadolinium partition coefficients) and showed a positive correlation between the 2 methods. ${ }^{14}$ However, no differences in collagen volume fraction or gadolinium distribution volumes were found between the 2 groups of myocardial fibrosis (increased interstitial fibrosis and infarcted myocardium). In an approach to investigate interstitial fibrosis in vivo in a patient with amyloidosis, Krombach et al. applied T1 quantification using a Look-Locker sequence. ${ }^{17}$ However, areas of fibrosis were already visible on late enhancement images in this case report. A pilot study was performed to detect interstitial fibrosis using T1-mapping in 8 patients with chronic aortic regurgitation, but no difference was found in myocardial T1 relaxation times between controls and patients. ${ }^{15}$ Only when segmental analysis was performed in segments with resting wall motion abnormalities, T1 relaxation times were different between controls and patients. It was concluded that this technique detected areas of focal fibrosis, but not interstitial fibrosis. Jerosch-Herold et al. suggested that patients with DCM show increased global myocardial accumulation of Gadolinium at steady state. ${ }^{16}$ They examined 9 patients and found that the myocardial partition coefficient for Gadolinium contrast was related to LV dilation and systolic dysfunction. However, these clinical MR imaging studies were not compared with biopsy. Iles et al. performed a study to assess interstitial fibrosis of the LV with MR imaging and used a prototype T1 mapping sequence in 25 patients with heart failure. ${ }^{18}$ Post-contrast LV T1 times correlated with interstitial fibrosis determined from RV biopsies.

Increased interstitial fibrosis has been described in DCM. ${ }^{3,19}$ It has been associated with an adverse prognosis or with impaired response to therapeutic interventions. Aoki et al. performed LV endomyocardial biopsies in 40 hemodialysis patients and 50 nondialysis patients with DCM. ${ }^{4}$ The extent of interstitial LV fibrosis was a strong predictor of cardiac death in this study. Bruckner et al. performed LV biopsy in 18 patients with LV assist device and LVEF $<20 \%{ }^{5}$ The group not responding to therapy showed an increased collagen volume fraction in comparison with the responding group. It has been suggested that the amount of interstitial fibrosis could potentially decrease with therapeutic interventions. In a group of 22 patients with severely impaired LV systolic function, the myocardial collagen content decreased during assist device therapy. ${ }^{20}$ Lopez et al stated that treatment with torasemide in 19 patients with systolic heart failure resulted in a reduction of the collagen volume fraction from $8 \pm 0.5 \%$ to $4.5 \pm 0.3 \%$ on RVbiopsies. ${ }^{6}$ It has been noted that in addition to interstitial fibrosis, focal fibrosis is also 
detectable on postmortem analysis in DCM. ${ }^{21,22}$ However, focal fibrosis as detected with LGE CMR was not related to the amount of interstitial fibrosis in our study.

Four observations are key to the interpretation of our study results. First, LV EDV and EF were not predictors of adverse outcome in other studies, the presence of focal fibrosis was a sole significant predictor of adverse outcome and ventricular tachycardia. ${ }^{1,2}$ Second, the impaired LV-function in our study subjects was due to global hypokinesia and not explained by regional hypokinesia only in myocardial segments showing focal fibrosis, and no correlation between LV and RV systolic dysfunction or dilation and focal fibrosis was found. Third, interstitial fibrosis was not related to LV and RV dilation or systolic dysfunction in our study. Fourth, focal fibrosis was related to inflammation. Thus, we postulate that 1) non-ischemic impaired systolic function could potentially be a reversible condition reacting to therapeutic interventions in patients with the presence of focal, but without excessive focal fibrosis, and 2) the formation of a focal arhythmogenic substrate with focal re-entry mechanisms could be the underlying process leading to ventricular tachycardia and sudden cardiac death in patients with nonischemic impaired systolic LV-function and focal fibrosis. ${ }^{23}$ While interstitial fibrosis could mainly lead to increased LV stiffness, reduced compliance, elevated fillingpressures, and, thus impairing both diastolic and systolic function, the presence of focal fibrosis could be associated with re-entry mechanisms in an area of scar formation leading to ventricular tachycardia and sudden death. A normal LGE CMR does not exclude increased interstitial fibrosis and, thus, standard LGE imaging is not a predictor of reactive fibrosis, but of adverse prognosis. ${ }^{1}$

We also postulate that 3) within the group of patients with DCM, myocarditis may have been the trigger for impaired systolic function in some cases, potentially explaining the transmural and epicardial patterns of LGE also found in the present study.

An unanswered question yet is whether guided biopsies from regions of interest could influence the diagnostic process. Mahrholdt et al suggested that biopsy guided by previous MR imaging could improve the diagnostic accuracy in patients with acute myocarditis. ${ }^{24}$ In a joined scientific statement from 3 heart associations, no recommendation was made for an approach that takes previous MR imaging for biopsy guiding purposes into account. Furthermore, it was concluded that no comparative study exists on which to base a recommendation for LV versus RV biopsy regarding procedural safety and diagnostic performance. ${ }^{25}$ However, one of the largest studies addressing the complication rate of endomyocardial biopsy in non-transplant patients confirmed a low complication rate for the femoral vein approach with RV biopsy. ${ }^{26}$ Currently, the most common biopsy site seems to be the RV side of the interventricular septum. ${ }^{25-28}$ To our knowledge, no study has been performed addressing the role of RV versus LV biopsy. Therefore, we performed a pilot study in autopsy hearts showing now differences between RV and LV biopsy samples. Van Suylen et al found that postmortem analysis of interstitial fibrosis of the LV free wall correlated well with endomyocardial biopsy of the right side of the intraventricular septum in transplanted human hearts. ${ }^{29}$ In the above 
mentioned study, aimed to assess interstitial fibrosis of the LV with MR imaging using a prototype T1 mapping sequence, post-contrast T1 times of the LV myocardium correlated with interstitial fibrosis determined from RV biopsies. ${ }^{18}$ The amount of RV fibrosis will not only increase if severe dilation or dysfunction of the RV is present. In our study, the amount of interstitial fibrosis determined from RV biopsy specimens was not related to LV and RV volume and function.

In conclusion, focal fibrosis detected with LGE CMR occurred independently from the amount of interstitial fibrosis determined from RV biopsy specimens. Both forms of fibrosis were not correlated with the degree of LV and RV dilation or systolic dysfunction. Focal fibrosis might be related to inflammation rather than interstitial fibrosis.

\section{References}

1. Assomull RG, Prasad SK, Lyne J, Smith G, Burman ED, Khan M, Sheppard MN, Poole-Wilson PA, Pennell DJ. Cardiovascular magnetic resonance, fibrosis, and prognosis in dilated cardiomyopathy. J Am Coll Cardiol 2006;48:1977-1985.

2. Wu KC, Weiss RG, Thiemann DR, Kitagawa K, Schmidt A, Dalal D, Lai S, Bluemke DA, Gerstenblith G, Marban E, Tomaselli GF, Lima JA. Late gadolinium enhancement by cardiovascular magnetic resonance heralds an adverse prognosis in nonischemic cardiomyopathy. J Am Coll Cardiol 2008;51:2414-2421.

3. Schwarz F, Mall G, Zebe H, Blickle J, Derks H, Manthey J, Kubler W. Quantitative morphologic findings of the myocardium in idiopathic dilated cardiomyopathy. Am J Cardiol 1983;51:501-506.

4. Aoki J, Ikari Y, Nakajima H, Mori M, Sugimoto T, Hatori M, Tanimoto S, Amiya E, Hara K. Clinical and pathologic characteristics of dilated cardiomyopathy in hemodialysis patients. Kidney Int 2005;67:333-340.

5. Bruckner BA, Razeghi P, Stetson S, Thompson L, Lafuente J, Entman M, Loebe M, Noon G, Taegtmeyer H, Frazier $\mathrm{OH}$, Youker K. Degree of cardiac fibrosis and hypertrophy at time of implantation predicts myocardial improvement during left ventricular assist device support. J Heart Lung Transplant 2004;23:36-42.

6. Lopez B, Querejeta R, Gonzalez A, Sanchez E, Larman M, Diez J. Effects of loop diuretics on myocardial fibrosis and collagen type I turnover in chronic heart failure. J Am Coll Cardiol 2004;43:2028-2035.

7. Heymans S, Schroen B, Vermeersch P, Milting H, Gao F, Kassner A, Gillijns H, Herijgers P, Flameng W, Carmeliet $P$, Van de Werf F, Pinto YM, Janssens S. Increased cardiac expression of tissue inhibitor of metalloproteinase- 1 and tissue inhibitor of metalloproteinase-2 is related to cardiac fibrosis and dysfunction in the chronic pressure-overloaded human heart. Circulation 2005;112:1136-1144.

8. Brooks A, Schinde V, Bateman AC, Gallagher PJ. Interstitial fibrosis in the dilated non-ischaemic myocardium. Heart 2003;89:1255-1256.

9. Montera MW, Drumond C, Takiya C, Mesquita CT, Dohmann HF, Mady C. Correlation of myocardial interstitial collagen in the right ventricular septum with ventricular function of patients with ischemic cardiomyopathy. Arq Bras Cardiol 2009;92:54-62.

10. Alfakih K, Plein S, Thiele H, Jones T, Ridgway JP, Sivananthan MU. Normal human left and right ventricular dimensions for MRI as assessed by turbo gradient echo and steady-state free precession imaging sequences. J Magn Reson Imaging 2003;17:323-329.

11. McCrohon JA, Moon JC, Prasad SK, McKenna WJ, Lorenz CH, Coats AJ, Pennell DJ. Differentiation of heart failure related to dilated cardiomyopathy and coronary artery disease using gadolinium-enhanced cardiovascular magnetic resonance. Circulation 2003;108:54-59. 
12. Bello D, Shah DJ, Farah GM, Di Luzio S, Parker M, Johnson MR, Cotts WG, Klocke FJ, Bonow RO, Judd RM, Gheorghiade M, Kim RJ. Gadolinium cardiovascular magnetic resonance predicts reversible myocardial dysfunction and remodeling in patients with heart failure undergoing beta-blocker therapy. Circulation 2003;108:1945-1953.

13. Adabag AS, Maron BJ, Appelbaum E, Harrigan CJ, Buros JL, Gibson CM, Lesser JR, Hanna CA, Udelson JE, Manning WJ, Maron MS. Occurrence and frequency of arrhythmias in hypertrophic cardiomyopathy in relation to delayed enhancement on cardiovascular magnetic resonance. J Am Coll Cardiol 2008;51:13691374.

14. Kehr E, Sono M, Chugh SS, Jerosch-Herold M. Gadolinium-enhanced magnetic resonance imaging for detection and quantification of fibrosis in human myocardium in vitro. Int J Cardiovasc Imaging 2008;24:61-68.

15. Sparrow P, Messroghli DR, Reid S, Ridgway JP, Bainbridge G, Sivananthan MU. Myocardial T1 mapping for detection of left ventricular myocardial fibrosis in chronic aortic regurgitation: pilot study. AJR Am J Roentgenol 2006;187:W630-635.

16. Jerosch-Herold M, Sheridan DC, Kushner JD, Nauman D, Burgess D, Dutton D, Alharethi R, Li D, Hershberger RE. Cardiac magnetic resonance imaging of myocardial contrast uptake and blood flow in patients affected with idiopathic or familial dilated cardiomyopathy. Am J Physiol Heart Circ Physiol 2008;295:H1234-H1242.

17. Krombach GA, Hahn C, Tomars M, Buecker A, Grawe A, Gunther RW, Kuhl HP. Cardiac amyloidosis: MR imaging findings and T1 quantification, comparison with control subjects. J Magn Reson Imaging 2007;25:1283-1287.

18. Iles L, Pfluger H, Phrommintikul A, Cherayath J, Aksit P, Gupta SN, Kaye DM, Taylor AJ. Evaluation of diffuse myocardial fibrosis in heart failure with cardiac magnetic resonance contrast-enhanced T1 mapping. J Am Coll Cardiol 2008;52:1574-1580.

19. de Leeuw N, Ruiter DJ, Balk AH, de Jonge N, Melchers WJ, Galama JM. Histopathologic findings in explanted heart tissue from patients with end-stage idiopathic dilated cardiomyopathy. Transpl Int 2001;14:299306.

20. Maybaum S, Mancini D, Xydas S, Starling RC, Aaronson K, Pagani FD, Miller LW, Margulies K, McRee S, Frazier $\mathrm{OH}$, Torre-Amione G. Cardiac improvement during mechanical circulatory support: a prospective multicenter study of the LVAD Working Group. Circulation 2007;115:2497-2505.

21. Waller TA, Hiser WL, Capehart JE, Roberts WC. Comparison of clinical and morphologic cardiac findings in patients having cardiac transplantation for ischemic cardiomyopathy, idiopathic dilated cardiomyopathy, and dilated hypertrophic cardiomyopathy. Am J Cardiol 1998;81:884-894.

22. Roberts WC, Siegel RJ, McManus BM. Idiopathic dilated cardiomyopathy: analysis of 152 necropsy patients. Am J Cardiol 1987;60:1340-1355.

23. Wu TJ, Ong JJ, Hwang C, Lee JJ, Fishbein MC, Czer L, Trento A, Blanche C, Kass RM, Mandel WJ, Karagueuzian HS, Chen PS. Characteristics of wave fronts during ventricular fibrillation in human hearts with dilated cardiomyopathy: role of increased fibrosis in the generation of reentry. J Am Coll Cardiol 1998;32:187196.

24. Mahrholdt H, Goedecke C, Wagner A, Meinhardt G, Athanasiadis A, Vogelsberg H, Fritz P, Klingel K, Kandolf R, Sechtem U. Cardiovascular magnetic resonance assessment of human myocarditis: a comparison to histology and molecular pathology. Circulation 2004;109:1250-1258.

25. Cooper LT, Baughman KL, Feldman AM, Frustaci A, Jessup M, Kuhl U, Levine GN, Narula J, Starling RC, Towbin J, Virmani R. The role of endomyocardial biopsy in the management of cardiovascular disease: a scientific statement from the American Heart Association, the American College of Cardiology, and the European Society of Cardiology Endorsed by the Heart Failure Society of America and the Heart Failure Association of the European Society of Cardiology. Eur Heart J 2007;28:3076-3093.

26. Holzmann M, Nicko A, Kuhl U, Noutsias M, Poller W, Hoffmann W, Morguet A, Witzenbichler B, Tschope C, Schultheiss HP, Pauschinger M. Complication rate of right ventricular endomyocardial biopsy via the femoral approach: a retrospective and prospective study analyzing 3048 diagnostic procedures over an 11-year period. Circulation 2008;118:1722-1728. 
27. Deckers JW, Hare JM, Baughman KL. Complications of transvenous right ventricular endomyocardial biopsy in adult patients with cardiomyopathy: a seven-year survey of 546 consecutive diagnostic procedures in a tertiary referral center. J Am Coll Cardiol 1992;19:43-47.

28. Felker GM, Hu W, Hare JM, Hruban RH, Baughman KL, Kasper EK. The spectrum of dilated cardiomyopathy. The Johns Hopkins experience with 1,278 patients. Medicine (Baltimore) 1999;78:270-283.

29. van Suylen RJ, van Bekkum EEC, Boersma H, de Kok LB, Balk AHMM, Bos E, Bosman FT. Collagen content and distribution in the normal and transplanted human heart: A postmortem quantitative light microscopic analysis. Cardiovascular Pathology 1996;5:61-68. 


\section{Right ventricular function in dilated cardiomyopathy and ischemic heart disease:}

Assessment with non-invasive imaging

S. Schalla, C. Jaarsma, S.C. Bekkers, J. Waltenberger, R. Dennert, H.J. Crijns, J. Wildberger, S. Heymans, H.P. Brunner-La Rocca. 


\section{Abstract}

Background: Dilated cardiomyopathy and ischaemic heart disease can both lead to right ventricular (RV) dysfunction. Direct comparisons of the two entities regarding RV size and function using state-of-the-art imaging techniques have not yet been performed. We aimed to determine RV function and volume in dilated cardiomyopathy and ischaemic heart disease in relation to left ventricular (LV) systolic and diastolic function and systolic pulmonary artery pressure.

Methods: A well-characterised group (cardiac magnetic resonance imaging, echocardiography, coronary angiography and endomyocardial biopsy) of 46 patients with dilated cardiomyopathy was compared with LV ejection fraction (EF)-matched patients $(n=23)$ with ischaemic heart disease. Volumes and EF were determined with magnetic resonance imaging, diastolic LV function and pulmonary artery pressure with echocardiography.

Results: After multivariable linear regression, four factors independently influenced $\operatorname{RVEF}\left(\mathrm{R}^{2}=0.51, p<0.001\right)$ : LVEF $(r=0.54, p<0.001)$, ratio of peak early and peak atrial transmitral Doppler flow velocity as measure of LV filling pressure $(r=-0.52, p<0.001)$ and tricuspid regurgitation flow velocity as measure of pulmonary artery pressure $(r=-0.38, p=0.001)$. RVEF was significantly worse in patients with dilated cardiomyopathy compared with ischaemic heart disease: median $48 \%$ (interquartile range (IQR) 37$55 \%$ ) versus $56 \%$ (IQR 48-63\%), $p<0.05$.

Conclusions: In patients with dilated cardiomyopathy and ischaemic heart disease, RV function is determined by LV systolic and diastolic function, the underlying cause of LV dysfunction, and pulmonary artery pressure. It was demonstrated that RV function is more impaired in dilated cardiomyopathy. 


\section{Introduction}

Right ventricular (RV) dysfunction and dilatation are correlated to limited exercise capacity and poor outcome, ${ }^{1-4}$ but often neglected in the clinical setting. ${ }^{5,6}$ Dilated cardiomyopathy (DCM) and ischemic heart disease (IHD) can both lead to RV dysfunction. Direct comparisons of the two entities with respect to RV size and function using stateof-the-art imaging techniques have not yet been performed. Such a comparison may help to better understand the underlying pathophysiology of RV dysfunction. Therefore, we determined RV function and volume in relation to left ventricular (LV) systolic and diastolic function, and pulmonary artery pressure in patients with DCM to assess the main mechanisms of RV dysfunction. In addition, after matching for LV ejection fraction (LVEF), patients with IHD due to infarction of the left coronary artery were also examined.

\section{Methods}

\section{Study population}

Two groups of patients were included in the study: patients with DCM from the Maastricht University Medical Center and patients with IHD from our infarct database matched for LVEF.

Study subjects in the DCM group comprised 46 consecutive patients with DCM referred to our heart failure program. All underwent a standard diagnostic evaluation including ECG, coronary angiography, endomyocardial biopsy, echocardiography and cardiac magnetic resonance imaging (CMR). LV and RV volumes and systolic function were determined by CMR. LV diastolic function and RV systolic pressure were assessed with echocardiography as described below. Patients with a history of myocardial infarction, history of cardiotoxic agents, significant coronary artery stenosis on coronary angiography, valvular heart disease on echocardiography as well as other known causes for impaired systolic function such as inflammation or infiltrative disorders on endomyocardial biopsy were excluded from the study. Patients with permanent pacemakers, rhythm other than sinus and significant chronic renal failure ( $\geq$ stage 3 kidney disease) were also not included.

Study subjects in the IHD group were included from our infarct data base matched for LV EF as measured with CMR. Thus, 23 patients with impaired systolic LV-function due to chronic non-inferior myocardial infarction (infarction in left anterior descending (LAD) or left circumflex (LCX) coronary artery territory to avoid inclusion of patients with RV infarction) and without significant valvular disease were included. The study was approved by the local ethics committee. 


\section{Magnetic resonance imaging}

Patients were examined with a 1.5 Tesla scanner (Gyroscan Intera, Philips Medical Systems, Best, the Netherlands). ECG-gated cine images were acquired for functional analysis using a steady-state free precession sequence (slice thickness $6 \mathrm{~mm}$, gap $4 \mathrm{~mm}$, TR/TE 3.8/1.9ms, flip angle 50 , FOV 350mm, matrix $256 \times 256,22-25$ phases per cardiac cycle). A breath hold multislice T1-weighted 3D inversion-recovery gradient-echo sequence (acquired/reconstructed slice thickness 12/6mm, average TR/TE 3.9/2.4ms, multi-shot [50 profiles/shot] segmented partial echo readout, flip angle $15^{\circ}$, FOV $400 \mathrm{~mm}$, matrix $256 \times 256$ ) to evaluate the presence of myocardial infarct of the LV and RV was used to acquire images 10 minutes after intravenous administration of $0.2 \mathrm{mmol} / \mathrm{kg}$ body weight Gadolinium diethylenetriaminepentaacetic acid (Magnevist, Bayer, Germany). Inversion times were adjusted to null normal myocardium (200280ms).

CMR images were analyzed by an investigator blinded to clinical and echocardiographic data. Endocardial and epicardial LV and endocardial RV contours were manually traced in end-diastolic and end-systolic phases on short axis slices to determine LV and RV end-diastolic volume (EDV), end-systolic volume (ESV), ejection fraction (EF) and LV end-diastolic mass. Areas of late enhancement were visually assessed to confirm the presence and extent of infarction in LAD- or LCX-territory as the reason for impaired LV EF in the IHD-group.

\section{Echocardiography}

Echocardiography was used to determine LV diastolic function, LA volume, and peak tricuspid regurgitation (TI) Doppler velocity. Transthoracic echocardiograms were performed using dedicated systems (Sonos 5500 with S3 or IE33 with S5-1 transducers, Philips Medical Systems, Andover, MA, USA). Echocardiographic investigations were performed in the standard views, according to the recommendations of the American Society of Echocardiography. ${ }^{7}$ LV diastolic function was assessed by measuring mitral (ratio of peak early and peak atrial transmitral Doppler flow velocity, E/A) and pulmonary vein flow velocities (ratio of peak systolic and diastolic pulmonary vein Doppler flow velocity, S/D), tissue Doppler flow velocities from the basal septal and lateral wall to calculate the ratio of early transmitral inflow and myocardial tissue velocity (E/e') and left atrial (LA) volumes. Since we did not include patients with normal LV-function, we used the E/A-ratio as an easily obtainable measure of LV filling pressure. Peak tricuspid regurgitation (TI) Doppler flow velocity was used as a measure of pulmonary artery systolic pressure (PAP). Images were digitally stored and analyzed off-line by an investigator blinded to CMR-results and clinical data. 


\section{Statistical analysis}

Variables are expressed as percentage, mean \pm standard deviation or median (interquartile range) as appropriate. The Kolmogorov-Smirnov test was applied to test if continuous variables were normally distributed. Group comparisons were performed by using the Pearson $\chi^{2}$ test, student's t-test or Mann-Whitney $U$ test, as appropriate. For correlations, Pearson $r$ was used. Finally, independent variables influencing RV EF were sought by using multivariable linear regression model using backward procedure (inclusion $\mathrm{p}<0.05$, exclusion $\mathrm{p}<0.1)$.

\section{Results}

\section{Clinical characteristics of patients with DCM and IHD}

The clinical characteristics of the DCM and IHD patients are summarized in table 1. Patients in the IHD group were older and less often treated with diuretics. All other parameters were not significantly different between the groups.

CMR image analysis was possible in all patients. In 3 patients, however, image quality on echocardiography was not sufficient to obtain reliable pulmonary vein flow Doppler signals. In 2 of them, image quality was also not sufficient to obtain tissue Doppler signals, but E/A-ratio could be determined in all patients.

A total of 21 patients with IHD had infarcts in the LAD-territory and 2 patients in the LAD- and LCX-territory (figure 1). Infarction of the RV free wall was recognized in only one patient of the IHD-group: a small area of late enhancement of the RV apex was visible on CMR images. This patient had a transmural infarction in the LAD-territory showing late enhancement from the anterior wall to the apex of the LV continuing to the RV apex (figure 2). None of the patients with DCM had focal fibrosis of the RV free wall myocardium on CMR late enhancement images. 
Table 1: Clinical, CMR and echocardiographic characteristics of the patients with dilated cardiomyopathy (DCM) and ischemic heart disease (IHD)

\begin{tabular}{|c|c|c|c|}
\hline Characteristic & DCM $(n=46)$ & $\mathrm{IHD}(\mathrm{n}=23)$ & P-value \\
\hline Age, years & $49 \pm 14$ & $59 \pm 16$ & $<0.01$ \\
\hline Male, n (\%) & $27(59 \%)$ & $18(78 \%)$ & 0.18 \\
\hline Body surface area, $\mathrm{m}^{2}$ & $1.92 \pm 0.25$ & $1.97 \pm 0.19$ & 0.28 \\
\hline Dyspnea & & & 0.48 \\
\hline NYHA 1/2, n (\%) & $38(83 \%)$ & $21(91 \%)$ & \\
\hline NYHA 3/4, n (\%) & $8(17 \%)$ & $2(9 \%)$ & \\
\hline Duration HF, months & $4(1-18)$ & $3(0-19)$ & 0.98 \\
\hline Diabetes mellitus & & & 0.62 \\
\hline DM type $1, \mathrm{n}(\%)$ & $1(2 \%)$ & $0(0 \%)$ & \\
\hline DM type 2, n (\%) & $4(9 \%)$ & $1(4 \%)$ & \\
\hline COPD, n (\%) & $6(13 \%)$ & $2(9 \%)$ & 0.71 \\
\hline Hypertension, n (\%) & $9(18 \%)$ & $8(35 \%)$ & 0.24 \\
\hline Systolic BP & $125 \pm 20$ & $123 \pm 19$ & 0.80 \\
\hline Diastolic BP & $75 \pm 12$ & $74 \pm 10$ & 0.75 \\
\hline Heart rate, beats/min & $77 \pm 14$ & $73 \pm 13$ & 0.32 \\
\hline PR duration, ms & $150 \pm 57$ & $157 \pm 60$ & 0.66 \\
\hline QRS duration, ms & $111 \pm 31$ & $92 \pm 43$ & 0.10 \\
\hline LBBB, n (\%) & $16(35 \%)$ & $4(17 \%)$ & 0.17 \\
\hline RBBB, n (\%) & $0(0 \%)$ & $2(9 \%)$ & 0.11 \\
\hline Creatinine, $\mu \mathrm{mol} / \mathrm{L}$ & $92 \pm 27$ & $95 \pm 45$ & 0.74 \\
\hline Beta-blocker & $36(78 \%)$ & $18(78 \%)$ & 0.34 \\
\hline ACE-inh. or AT-II-blocker & $40(87 \%)$ & 21 (91\%) & 0.20 \\
\hline Diuretic & $32(70 \%)$ & 9 (39\%) & 0.03 \\
\hline Aldosteron antagonist & $12(26 \%)$ & $4(17 \%)$ & 0.28 \\
\hline Ca-channel blocker & $0(0 \%)$ & $1(4 \%)$ & 0.13 \\
\hline RV EDV, $\mathrm{ml} / \mathrm{m} 2$ & $78(65-92)$ & $71(63-78)$ & 0.03 \\
\hline RV ESV, $\mathrm{ml} / \mathrm{m} 2$ & $41(35-51)$ & 32 (24-39) & 0.03 \\
\hline $\mathrm{RV} \mathrm{SV}, \mathrm{ml} / \mathrm{m} 2$ & $35(28-43)$ & $38(29-43)$ & 0.98 \\
\hline$R V E F, \%$ & $48(37-55)$ & $56(48-63)$ & 0.05 \\
\hline LV EDV, $\mathrm{ml} / \mathrm{m}^{2}$ & $120(96-158)$ & $131(101-165)$ & 0.79 \\
\hline LV ESV, $\mathrm{ml} / \mathrm{m}^{2}$ & $82(64-117)$ & $85(66-128)$ & 0.63 \\
\hline LV SV, $\mathrm{ml} / \mathrm{m}^{2}$ & $39(29-46)$ & $38(34-43)$ & 0.75 \\
\hline LVEF, \% & $31(22-40)$ & $34(18-39)$ & 0.77 \\
\hline LV mass, $\mathrm{gr} / \mathrm{m}^{2}$ & $75(62-84)$ & $68(62-86)$ & 0.86 \\
\hline RA volume, $\mathrm{ml} / \mathrm{m}^{2}$ & $20(16-32)$ & $22(17-26)$ & 0.43 \\
\hline LA volume, $\mathrm{ml} / \mathrm{m}^{2}$ & $32(26-53)$ & $37(28-46)$ & 0.86 \\
\hline $\mathrm{E}$ max velocity, $\mathrm{cm} / \mathrm{s}$ & $71(55-82)$ & $68(53-98)$ & 0.67 \\
\hline$E / A$ & $1.00(0.70-1.40)$ & $0.96(0.63-1.78)$ & 0.99 \\
\hline dt E-top ms & $170(130-205)$ & $160(130-220)$ & 0.68 \\
\hline$S / D$ & $1.04(0.81-1.33)$ & $1.00(0.62-1.45)$ & 0.79 \\
\hline E/e'IVS & $10.0(8.0-13.0)$ & $6.5(4.9-7.5)$ & $<0.001$ \\
\hline E/e' lateral & $7.7(5.4-9.7)$ & $8.4(6.6-11.5)$ & 0.31 \\
\hline TI peak velocity, $\mathrm{m} / \mathrm{s}$ & $2.13(1.86-2.44)$ & $2.42(2.02-3.03)$ & 0.04 \\
\hline
\end{tabular}


Values represent mean \pm standard deviation, median (interquartile range) or $n$, numbers of patients (\%). DCM denotes dilated cardiomyopathy, IHD ischemic heart disease, NYHA New York Heart Association class, COPD chronic obstructive pulmonary disease, BP blood pressure, PR duration between $P$ and $R$ wave on ECG, QRS duration of QRS complex, LBBB left bundle branch block, RBBB right bundle branch block, ACE-inh or AT-IIblocker angiotensin converting enzyme inhibitor or angiotensin II receptor blocker, CA calcium, RV right ventricle, LV left ventricle, EDV end-diastolic volume, ESV end-systolic volume, SV stroke volume, EF ejection fraction, RA right atrium, LA left atrium, E peak early transmitral Doppler flow velocity, E/A ratio of peak early and peak atrial transmitral Doppler flow velocity, dt E-top deceleration time of peak early transmitral Doppler flow signal, S/D ratio of peak systolic and diastolic pulmonary vein Doppler flow verlocity, E/e' IVS ratio of peak early transmitral Doppler flow velocity and peak early diastolic tissue Doppler flow velocity from the basal septal left ventricular wall, E/e' lateral ratio of peak early transmitral Doppler flow velocity and peak early diastolic tissue Doppler flow velocity from the basal lateral left ventricular wall, TI peak tricuspid regurgitation Doppler flow velocity
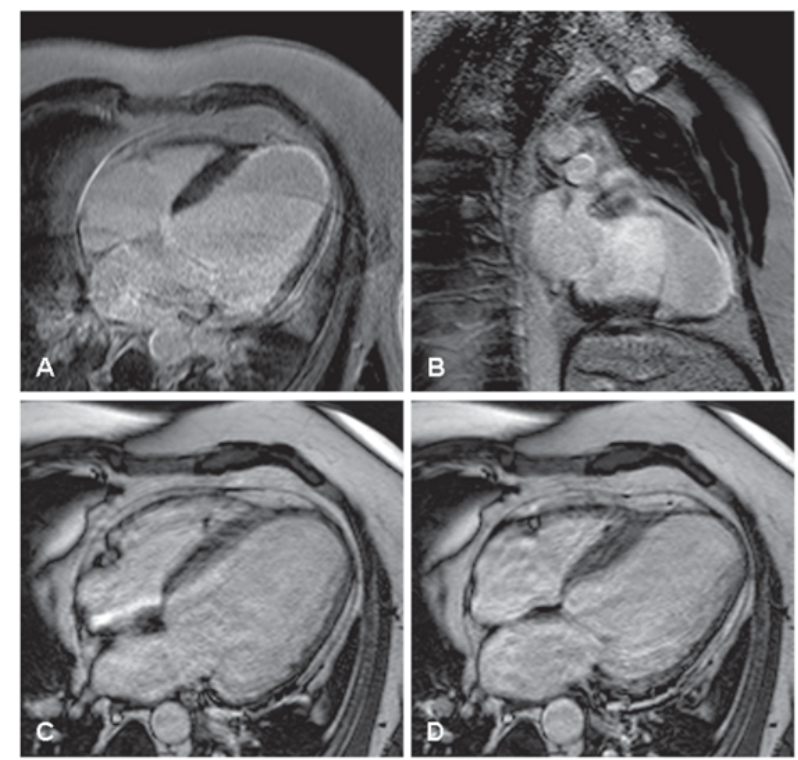

Figure 1: Late gadolinium enhancement (A, B) and cine (4 chamber view in end-diastole, $C$ and end-systole, D) magnetic resonance images of a patient with a large myocardial infarction (left ventricular ejection fraction $15 \%$ ) of the left anterior descending coronary artery (right ventricular ejection fraction 56\%). 

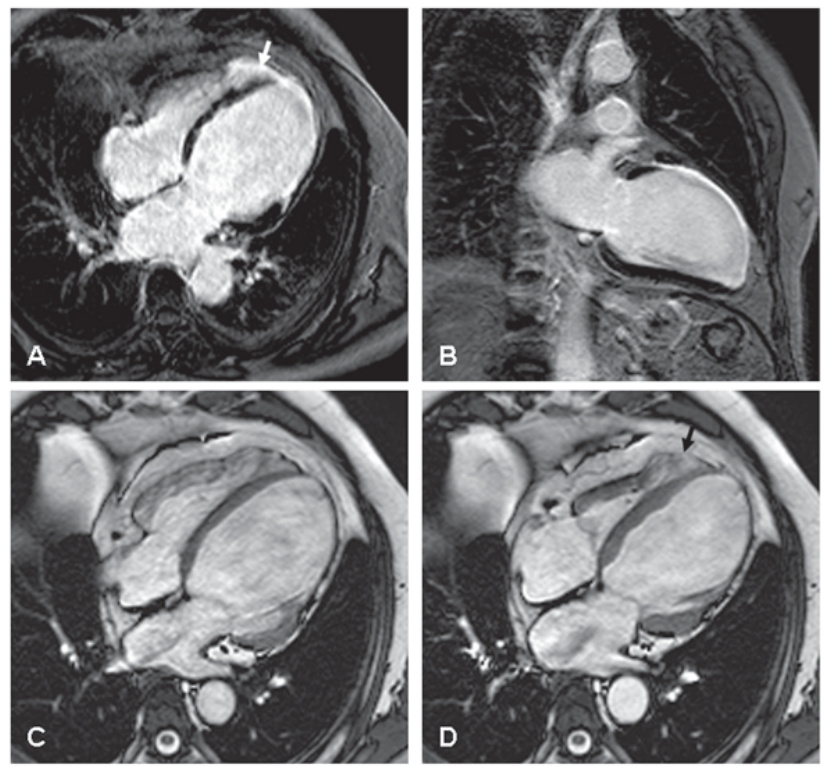

Figure 2: Late gadolinium enhancement (4 chamber view, A; 2 chamber view, B) and cine (4 chamber view in end-diastole, $C$ and end-systole, D) magnetic resonance images of a patient with a large myocardial infarction (left ventricular ejection fraction 22\%) of the left anterior descending coronary artery, continuing from the left ventricular apex to the right ventricle (right ventricular ejection fraction 57\%) (white arrow). A corresponding akinetic region of the right ventricular apex can be depicted on the end-systolic cine image ( $D$, black arrow).

\section{$R V$ volume and function}

\section{-Differences between patients with DCM and IHD}

The RV EF was more impaired and RV size larger in patients with DCM in comparison with patients with IHD (figures 3 and 4) despite similar LV systolic and diastolic dysfunction. There was an isolated increase in E/e' of the septum in DCM patients, but an opposite trend lateral and no further differences were seen in diastolic parameters between the groups, suggesting no difference in diastolic LV function. LV EDV and ESV were not different between the groups. All CMR and echocardiography parameters are summarized in table 1. 

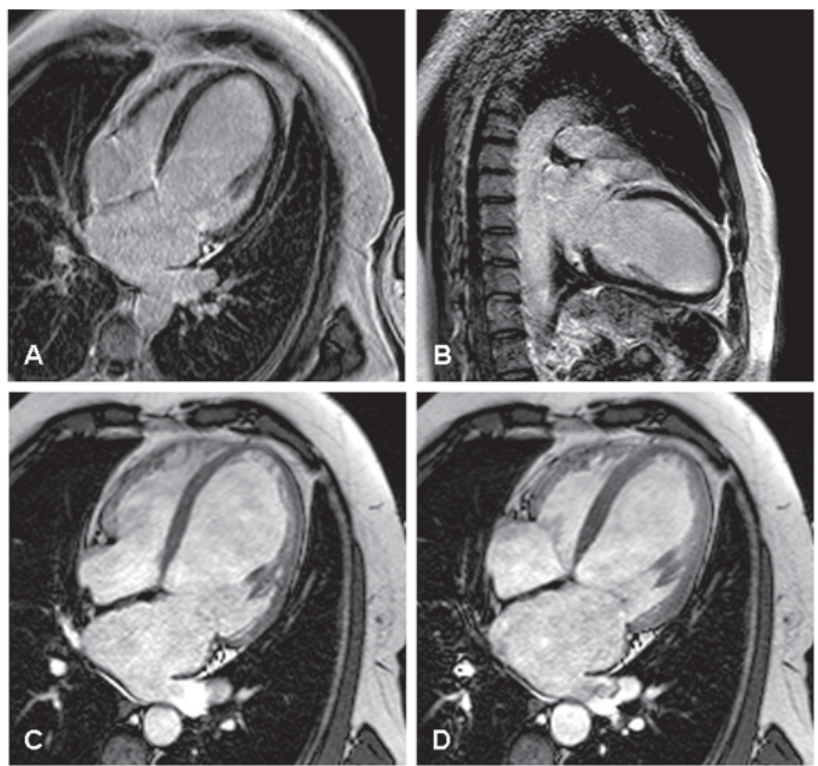

Figure 3: Late gadolinium enhancement (A, B) and cine (4 chamber view in end-diastole, $\mathrm{C}$ and end-systole, D) magnetic resonance images of a patient with idiopathic dilated cardiomyopathy (left ventricular ejection fraction $24 \%$, right ventricular ejection fraction $45 \%$ ).

The left ventricular systolic function of this patient is slightly better than those of the patients with ischemic heart disease from figure 1 and 2 while the right ventricular function is more impaired. Areas of late enhancement are not present.

\section{-Parameters influencing the RV function}

As depicted in table 2, the RV EF was significantly correlated with LV EF and the volumes of all four cardiac chambers. Furthermore, parameters of LV diastolic function negatively correlated with RV function. Also, the TI velocity as measure of PAP was negatively correlated with RV EF. Finally, age and heart rate showed significant correlations with RV EF. RV EF was higher in women compared with men ( $55 \pm 10$ versus $44 \pm 13 \%$, $p=0.001$ ). Other clinical parameters had no influence on RV EF (data not shown). Overall, there were no significant differences in these correlations between patients with DCM and IHD (data not shown). 

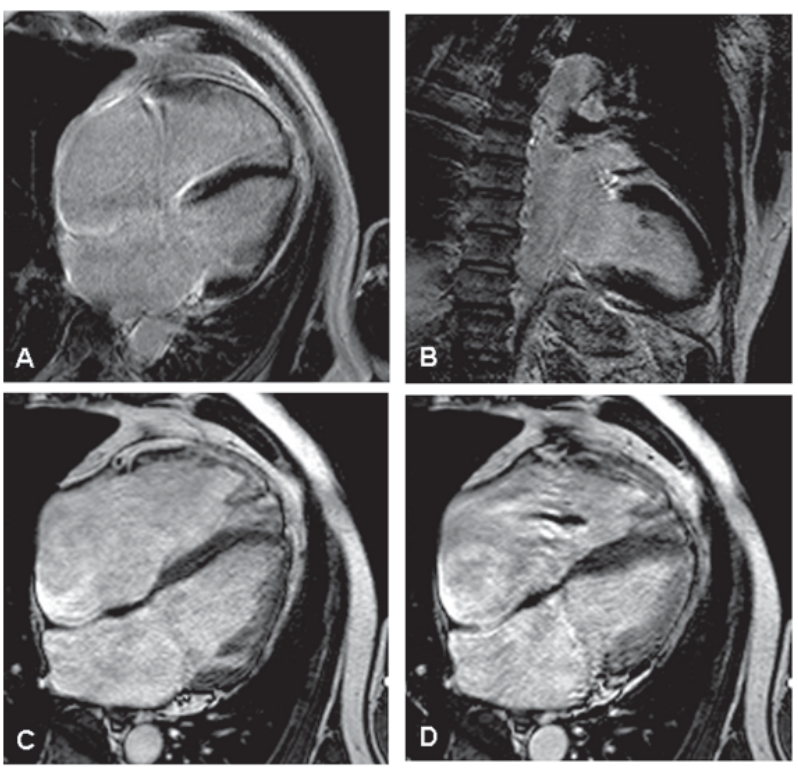

Figure 4: Late gadolinium enhancement (A, B) and cine (4 chamber view in end-diastole, $C$ and end-systole, D) magnetic resonance images of a patient with idiopathic dilated cardiomyopathy with more pronounced dilation of the right ventricle (left ventricular ejection fraction $36 \%$, end-diastolic volume $211 \mathrm{ml}$; right ventricular ejection fraction $23 \%$, end-diastolic volume $269 \mathrm{ml}$ ).

Although elevated left ventricular filling pressure and TI velocity were present, the degree of right ventricular dilation is out of proportion, possibly suggesting an active unknown process affecting the right ventricle more than the left ventricle.

Multivariable analysis revealed that the RV function (i.e. RV EF) was correlated with LV EF, DCM compared with IHD, E/A-ratio as measure of LV filling pressure, and TI-velocity as measure of PAP as depicted in table 3. Although the relationship with TI-velocity was of borderline significance, it was in addition to a measure of LV filling pressure, e.g. a possible active process in addition to passive elevation of PAP due to elevated LV filling pressures. 
Table 2: Correlations of clinical, CMR and echocardiographic characteristics with right ventricular ejection fraction as measured with CMR

\begin{tabular}{lcc}
\hline Variable & Correlation & P-value \\
\hline Age & 0.29 & 0.02 \\
Systolic BP & 0.13 & 0.28 \\
HR & -0.46 & $<0.001$ \\
LV EF & 0.54 & $<0.001$ \\
LV EDV & -0.29 & 0.02 \\
RV EDV & -0.50 & $<0.001$ \\
LA volume & -0.41 & $<0.001$ \\
RA volume & -0.37 & $<0.001$ \\
E/A ratio & -0.52 & $<0.001$ \\
S/D ratio & 0.48 & $<0.001$ \\
TI velocity & -0.38 & 0.001 \\
\hline
\end{tabular}

BP denotes systolic blood pressure, HR heart rate, LV left ventricle, EF ejection fraction, EDV end-diastolic volume, LA left atrium, RA right atrium, E/A ratio of peak early and peak atrial transmitral Doppler flow velocity, S/D ratio of peak systolic and diastolic pulmonary vein Doppler flow velocity, $\mathrm{TI}$ peak tricuspid regurgitation Doppler flow velocity

Table 3: Regression analysis testing the association between right ventricular ejection fraction and various potential predictors in the entire study population (dilated cardiomyopathy and ischemic heart disease)

\begin{tabular}{lcc}
\hline Variable & Regression coefficient & P-value \\
\hline LV EF (per \%) & 0.50 & $<0.0001$ \\
DCM vs. IHD & 9.41 & 0.0005 \\
E/A ratio (per unit) & -3.60 & 0.02 \\
TI-velocity (per m/s) & -4.84 & 0.06
\end{tabular}

$\left(R^{2}=0.51\right)$

DCM denotes dilated cardiomyopathy, IHD ischemic heart disease, LV left ventricle, ESV end-systolic volume, E/A ratio of peak early and peak atrial transmitral Doppler flow velocity, TI peak tricuspid regurgitation Doppler flow velocity.

\section{Discussion}

In the current study, the non-invasive imaging techniques CMR and echocardiography were applied in patients with DCM and IHD without RV free wall infarction, to evaluate RV function and volumes. RV systolic function was influenced by different factors including the underlying disease process, i.e. the presence of DCM, systolic and diastolic function of the LV and elevation of the PAP.

In ST-segment elevation myocardial infarction (STEMI) patients with cardiogenic shock, RV dysfunction had been identified as a prognostic parameter. ${ }^{8}$ Between patients with and without RV dysfunction, no significant differences in infarct related artery and infarct location were observed. RV dysfunction in patients in whom the RCA 
was not the infarct related artery was also present. This suggests that not only direct RV infarction causes RV dysfunction but also mechanisms such as reduced blood supply of the septum, low RV preload due to low output, and diminished contribution of LV contraction to RV systole. ${ }^{8}$ RV function may depend on LV septal contractile contribution transmitted through systolic ventricular interaction ${ }^{9-11}$ and the septum itself may contribute to the systolic function of both ventricles. ${ }^{12}$ RV function was experimentally as much impaired with septal ligation as with RCA ligation. ${ }^{13}$ Our results, however, suggest that other factors such as elevated LV filling pressure and PAP are of additional importance to explain reduced RV function.

RV infarction detected by late enhancement CMR and RV systolic function were outcome predictors in a general population of STEMI patients. ${ }^{14}$ In inferior STEMI, RV systolic function was related to the presence and extent of RV infarction while in anterior STEMI it was related to LV systolic dysfunction. When imaging more early after infarction, RV infarction was not related to prognosis. ${ }^{15}$ This discrepancy could be explained by the fact that RV function may recover to a greater extent than LV function, depending on recovery of septal function. ${ }^{9}$ Thus, global RV performance recovered within days after infarction, regardless of artery patency. ${ }^{16}$ Moreover, global RV performance improved greatly even in chronic RCA occlusion, despite persistent severe RV free wall dysfunction. ${ }^{17}$ In order to exclude the influence of direct RV free wall infarct on RV function, we included only patients with infarcts of the left coronary artery territory (LAD and LCX infarct). To our knowledge, this has not been applied in previous comparative studies of patients with DCM and IHD. We observed a small extension of infarct from the left to the RV myocardium in one patient only, which is in some contrast to a recent CMR-study in 20 patients with reperfused proximal LAD occlusion where a small RV infarct was observed in $40 \%$ of the patients. ${ }^{18}$ They found a relatively large area of the RV at risk for necrosis, although the resulting final RV infarction size was small. A possible explanation for the discrepancy between this and our findings is that only a small portion of our patients had transmural infarcts of the whole septum.

The underlying process also determines RV function. Patients with DCM experienced larger RV volumes and more severe impairment of RV function. In contrast to previous suggestions, ${ }^{19,20}$ assessing systolic RV-function alone is not sufficient to distinguish between DCM and IHD in individual patients. Few studies directly compared the RV function in patients with DCM and IHD. Our findings are supported by some earlier studies where patients with DCM showed more severe RV dysfunction than patients with IHD using radionuclide angiography, thermodilution and invasive RV angiography. ${ }^{19-21}$ However, these findings have not been uniform. By applying tissue Doppler imaging, the RV dysfunction was more pronounced in patients with IHD than DCM in an echocardiographic study. ${ }^{22}$ Since only 2 patients had inferior wall infarction based on ECG-criteria, the authors concluded that this worse RV function had not been due to more infarcted RV myocardium. However, patients were not sufficiently matched with respect to other factors potentially influencing RV function. Thus, patients with IHD 
exhibited more severe LV diastolic dysfunction and higher PAP. Such a difference in PAP and diastolic function was not present in our patient groups, but PAP and diastolic function were independently related to RV dysfunction. Moreover, assessment of RV dimensions and systolic function by echocardiography has important limitations. In the present study, we therefore used the current reference standard CMR to assess RV volumes and $E F$. ${ }^{6,23,24}$

Elevated LV filling pressure, which leads to passive elevation of PAP, was one of the factors related to RV dysfunction in the current study. Since the prognosis of patients with impaired RV function is worse in comparison to patients with impaired systolic LV function only, ${ }^{25-26}$ assessment of RV function and possibly more aggressive treatment of elevated LV filling pressure as suggested by Stevenson et al. might be important, irrespective of the underlying cause. ${ }^{27}$ An additional active component of elevated pulmonary artery pressure, i.e. out-of-proportion pulmonary hypertension leading to fixed pulmonary hypertension, may also have contributed to worsening of RV function since elevated PAP was related to RV dysfunction independently of LV filling pressure.

The current study has some limitations. Patients in the IHD group were older and less often treated with diuretics. Older age had a negative influence on RV function. However, although the patients in the IHD group were older, they showed a less impaired RV function than the patients from the DCM group. Thus, not matching for age did not result in a relevant bias. Treatment with diuretics can lower LV filling pressure and PAP and might have had an influence on the lower TI peak velocity measured in patients with DCM. ${ }^{28}$ In contrast to IHD, DCM usually comprises a heterogeneous group of diseases in many studies. However, with a comprehensive diagnostic routine including endomyocardial biopsy, imaging and blood tests, certain diseases such as acute myocarditis, infiltrative and storage diseases were excluded from our study to make the DCM study population as uniform as possible. Regional RV wall motion as measured by e.g. speckle tracking was not assessed. Early stages of dyssynchronicity between RV and LV were therefore not detected. Other potential mechanisms were also not addressed, e.g. interventricular interaction and effects of changes of geometry. Finally, this was a diagnostic study. However, the study revealed different potential mechanisms of RV dysfunction that may be therapeutically influenced. It is important to evaluate RV function, especially in DCM, and it may be necessary to treat elevated filling pressure and PAP more aggressively or with new therapeutic strategies such as phosphodiesterase inhibitors in order to protect the right ventricle.

In conclusion, RV systolic function was influenced by different factors including the underlying disease process, i.e. the presence of DCM, systolic and diastolic function of the LV and elevation of PAP. A better understanding of these mechanisms may help to define therapeutic targets for future studies in these patients with RV dysfunction known to have a poor outcome. 


\section{References}

1. Baker BJ, Wilen MM, Boyd CM, et al. Relation of right ventricular ejection fraction to exercise capacity in chronic left ventricular failure. Am J Cardiol 1984;54:596-599.

2. Di Salvo TG, Mathier M, Semigran MJ, et al. Preserved right ventricular ejection fraction predicts exercise capacity and survival in advanced heart failure. J Am Coll Cardiol 1995;25:1143-1153.

3. Gavazzi A, Berzuini C, Campana C, et al. Value of right ventricular ejection fraction in predicting shortterm prognosis of patients with severe chronic heart failure. J Heart Lung Transplant 1997;16:774-785.

4. de Groote P, Millaire A, Foucher-Hossein C, et al. Right ventricular ejection fraction is an independent predictor of survival in patients with moderate heart failure. J Am Coll Cardiol 1998;32:948-954.

5. Jefferies JL, Towbin JA. Dilated cardiomyopathy. Lancet 2010;375:752-762.

6. Mertens LL, Friedberg MK. Imaging the right ventricle-current state of the art. Nat Rev Cardiol 2010;7:551-563.

7. Gottdiener JS, Bednarz J, Devereux R, et al. American Society of Echocardiography recommendations for use of echocardiography in clinical trials. J Am Soc Echocardiogr 2004;17:1086-1119.

8. Engstrom AE, Vis MM, Bouma BJ, et al. Right ventricular dysfunction is an independent predictor for mortality in ST-elevation myocardial infarction patients presenting with cardiogenic shock on admission. Eur J Heart Fail 2010;12:276-282.

9. Popescu BA, Antonini-Canterin F, Temporelli PL, et al. Right ventricular functional recovery after acute myocardial infarction: relation with left ventricular function and interventricular septum motion. GISSI-3 echo substudy. Heart 2005;91:484-488.

10. Goldstein JA, Tweddell JS, Barzilai B, et al. Importance of left ventricular function and systolic ventricular interaction to right ventricular performance during acute right heart ischemia. J Am Coll Cardiol 1992;19:704-711.

11. Sharkey SW, Shelley W, Carlyle PF, et al. M-mode and two-dimensional echocardiographic analysis of the septum in experimental right ventricular infarction: correlation with hemodynamic alterations. Am Heart J 1985;110:1210-1218.

12. Banka VS, Agarwal JB, Bodenheimer MM, et al. Interventricular septal motion: biventricular angiographic assessment of its relative contribution to left and right ventricular contraction. Circulation 1981;64:992996.

13. Fixler DE, Monroe GA, Wheeler JM. Hemodynamic alterations during septal or right ventricular ischemia in dogs. Am Heart J 1977;93:210-215.

14. Miszalski-Jamka T, Klimeczek P, Tomala M, et al. Extent of RV dysfunction and myocardial infarction assessed by CMR are independent outcome predictors early after STEMI treated with primary angioplasty. JACC Cardiovasc Imaging 2010;3:1237-1246.

15. Hombach V, Grebe O, Merkle N, et al. Sequelae of acute myocardial infarction regarding cardiac structure and function and their prognostic significance as assessed by magnetic resonance imaging. Eur Heart J 2005;26:549-557.

16. Steele $\mathrm{P}$, Kirch D, Ellis J, et al. Prompt return to normal of depressed right ventricular ejection fraction in acute inferior infarction. Br Heart J 1977;39:1319-1323.

17. Laster SB, Shelton TJ, Barzilai B, et al. Determinants of the recovery of right ventricular performance following experimental chronic right coronary artery occlusion. Circulation 1993;88:696-708.

18. Bodi V, Sanchis J, Mainar L, et al. Right ventricular involvement in anterior myocardial infarction: a translational approach. Cardiovasc Res 2010;87:601-608.

19. Iskandrian AS, Helfeld H, Lemlek J, et al. Differentiation between primary dilated cardiomyopathy and ischemic cardiomyopathy based on right ventricular performance. Am Heart J 1992;123:768-773.

20. La Vecchia L, Zanolla L, Varotto L, et al. Reduced right ventricular ejection fraction as a marker for idiopathic dilated cardiomyopathy compared with ischemic left ventricular dysfunction. Am Heart J 2001;142:181-189. 
21. Juilliere $Y$, Buffet $P$, Marie PY, et al. Comparison of right ventricular systolic function in idiopathic dilated cardiomyopathy and healed anterior wall myocardial infarction associated with atherosclerotic coronary artery disease. Am J Cardiol 1994;73:588-590.

22. Parcharidou DG, Giannakoulas G, Efthimiadis GK, et al. Right ventricular function in ischemic or idiopathic dilated cardiomyopathy. Circ J 2008;72:238-244.

23. Doesch C, Zompolou C, Streitner F, et al. CMR-derived TAPSE measurement: a semi-quantitative method of right ventricular function assessment in patients with hypertrophic cardiomyopathy. Neth Heart J. 2014;22:557-564.

24. Quick S, Speiser U, Kury K, et al. Evaluation and classification of right ventricular wall motion abnormalities in healthy subjects by 3-tesla cardiovascular magnetic resonance imaging. Neth Heart J. 2015;23:6469.

25. Ghio S, Gavazzi A, Campana C, et al. Independent and additive prognostic value of right ventricular systolic function and pulmonary artery pressure in patients with chronic heart failure. J Am Coll Cardiol 2001;37:183-188.

26. Zornoff LA, Skali H, Pfeffer MA, et al. Right ventricular dysfunction and risk of heart failure and mortality after myocardial infarction. J Am Coll Cardiol 2002;39:1450-1455.

27. Stevenson LW, Dracup KA, Tillisch JH. Efficacy of medical therapy tailored for severe congestive heart failure in patients transferred for urgent cardiac transplantation. Am J Cardiol 1989;63:461-464.

28. Stampfer M, Epstein SE, Beiser GD, et al. Hemodynamic effects of diuresis at rest and during intense upright exercise in patients with impaired cardiac function. Circulation. 1968;37:900-911. 



\section{Structural and functional cardiac changes in myotonic dystrophy type 1 : \\ A cardiovascular magnetic resonance study}

M.C. Hermans, C.G. Faber, S.C: Bekkers, C.E. de Die-Smulders, M.M. Gerrits,

I.S. Merkies, G. Snoep, Y.M. Pinto, S. Schalla.

J Cardiovasc Magn Reson. 2012 Jul 24;14:48. 


\section{Abstract}

Background: Myotonic dystrophy type 1 (MD1) is a neuromuscular disorder with potential involvement of the heart and increased risk of sudden death. Considering the importance of cardiomyopathy as a predictor of prognosis, we aimed to systematically evaluate and describe structural and functional cardiac alterations in patients with MD1.

Methods: Eighty MD1 patients underwent physical examination, electrocardiography (ECG), echocardiography and cardiovascular magnetic resonance (CMR). Blood samples were taken for determination of NT-proBNP plasma levels and CTG repeat length.

Results: Functional and structural abnormalities were detected in 35 patients (44\%). Left ventricular systolic dysfunction was found in 20 cases, left ventricular dilatation in 7 patients, and left ventricular hypertrophy in 6 patients. Myocardial fibrosis was seen in 10 patients (12.5\%). In general, patients had low left ventricular mass indexes. Right ventricular involvement was uncommon and only seen together with left ventricular abnormalities. Functional or structural cardiac involvement was associated with age $(p=$ $0.04)$, male gender $(p<0.001)$ and abnormal ECG $(p<0.001)$. Disease duration, CTG repeat length, severity of neuromuscular symptoms and NT-proBNP level did not predict the presence of myocardial abnormalities.

Conclusions: CMR can be useful to detect early structural and functional myocardial abnormalities in patients with MD1. Myocardial involvement is strongly associated with conduction abnormalities, but a normal ECG does not exclude myocardial alterations. These findings lend support to the hypothesis that MD1 patients have a complex cardiac phenotype, including both myocardial and conduction system alteration. 


\section{Introduction}

Myotonic dystrophy type 1 (MD1), or Steinert's disease, is an autosomal dominant inherited disorder caused by an unstable expansion of a repetitive trinucleotide sequence (CTG) on chromosome 19. The prevalence varies from 2.1-14.3 per $100000 .{ }^{1}$ MD1 is characterized by slowly progressive weakness of skeletal muscles, myotonia and involvement of several organ systems. ${ }^{1}$ An earlier age of onset and increased severity of clinical symptoms has been observed in subsequent generations and is related to degree of CTG expansion. ${ }^{2}$

Patients with MD1 usually die from respiratory or cardiac complications., ${ }^{3,4}$ Sudden death is considered to be the result of atrioventricular block or ventricular arrhythmias. ${ }^{5}$ Recent studies showed that severe electrocardiographic (ECG) abnormalities and atrial arrhythmias are independent risk factors, although with moderate sensitivity, for sudden death in MD1 patients. ${ }^{6}$ Although death from progressive heart failure is uncommon in patients with MD1 compared to other muscular dystrophies, ${ }^{7,8}$ left ventricular systolic dysfunction is associated with an increased risk of overall mortality and sudden death. ${ }^{9}$ Therefore, the picture emerges that MD1 patients have a complex cardiac phenotype including both the myocardium and the conduction system.

Considering the importance of cardiomyopathy as a predictor of prognosis, we aimed to measure cardiac function and detect structural abnormalities in patients with MD1. We used cardiovascular magnetic resonance (CMR) in the current study since it is an accurate and highly reproducible technique for the assessment of cardiac volumes, function, mass and focal fibrosis and the interstudy reproducibility in normal, dilated, and hypertrophic hearts was superior to 2-dimensional echocardiography. ${ }^{10}$

\section{Methods}

\section{Patient selection}

The protocol was approved by the local Medical Ethics Committee and each participant gave written informed consent. Patients older than 18 years of age were invited for a prospective, on-going study on cardiac involvement and early stratification of arrhythmogenic risk. Participants were recruited from the genetic register of the Maastricht University Medical Centre and through the Dutch neuromuscular patients' association (Vereniging Spierziekten Nederland, VSN). Subjects with previously implanted pacemakers or implantable cardioverter-defibrillators or with severe comorbidity leading to reduced life expectancy such as malignant disease or respiratory failure, were excluded. During a 2 year period, 80 consecutive patients underwent CMR imaging and were enrolled in this study. 


\section{Clinical examination}

A standardized interview was conducted in all participants to evaluate their clinical history and current symptoms. A neurological and cardiac evaluation was conducted by the same examiner $(\mathrm{MH})$ in a predefined standardized fashion. The MD1 phenotype was established according to the commonly accepted classification based on the age at onset of symptoms: mild (late onset), classical (adult onset) and childhood/congenital type. ${ }^{2}$ Skeletal muscle strength was manually tested and graded according to the Medical Research Council (MRC) 6-point grading system (0-5). ${ }^{11} \mathrm{~A}$ total of 22 muscle groups were tested: neck flexors and extensors separately plus 10 bilateral muscles: 6 proximal muscle groups (shoulder abductors, elbow flexors, elbow extensors, hip flexors, knee extensors, knee flexors) and 4 distal muscle groups (wrist extensors, digits flexors, ankle dorsiflexors, ankle plantar flexors). Summation of the scores yields an extended MRCsumscore, ranging from 0 (paralytic) to 110 (normal strength). ${ }^{12,13}$ Furthermore, blood samples were taken to determine N-Terminal pro Brain Natriuretic Peptide (NT-proBNP) plasma levels and the length of the CTG repeat.

\section{CTG repeat lengths analysis}

Analysis of the MD1 CTG repeat length was performed on peripheral blood lymphocytes. Polymerase chain reaction followed by fragment length analysis was used to determine small allele lengths of 5 to 100 repeats, and Southern blotting was used to estimate repeat lengths $>100$. For purposes of statistical analysis, the CTG expansions were divided in 4 categories (<100; 100-250; 250-500; >500).

\section{Electrocardiography}

ECG was considered abnormal if signs of conduction disease (PR interval $\geq 210 \mathrm{~ms}$, QRS duration $\geq 120 \mathrm{~ms}$, left anterior or posterior fascicular hemiblock), hypertrophy (Sokolow-Lyon index $\geq 35 \mathrm{~mm}$ ), myocardial infarction or rhythm other than sinus, were present.

\section{Echocardiography}

Echocardiography was used to exclude significant valvular disease, elevated right ventricular systolic pressure. Transthoracic echocardiograms were performed using a SONOS 5500 system with S3 transducer (Philips Medical Systems, Best, the Netherlands). Echocardiographic investigations were performed according to the recommendations of the American Society of Echocardiography. 
Patients were examined in supine position with a clinical 1.5 T Gyroscan Intera MR scanner (Philips Medical Systems, Best, the Netherlands) equipped with a 5 channel cardiac surface coil. ECG-gated cine images were acquired for functional analysis during multiple breath holds (10-13 seconds) using a steady-state free precession sequence (slice thickness $6 \mathrm{~mm}$, slice gap $4 \mathrm{~mm}$, TR/TE 3.8/1.9 ms, flip angle 50', FOV $350 \mathrm{~mm}$, matrix $256 \times 256,22-25$ phases per cardiac cycle) in two-chamber, three-chamber and four-chamber view and a short-axis stacks covering the entire LV. For the detection of myocardial edema multislice short axis images were acquired using a dual-inversion black-blood T2-weighted sequence with fat suppression (slice thickness $8 \mathrm{~mm}$, slice gap $2 \mathrm{~mm}$, TR/TE 1600/100 ms, flip angle 90, FOV 350 mm, matrix 512 x 512). After intravenous contrast administration (Gd-DTPA $0.2 \mathrm{mmol} / \mathrm{kg}$ ) a Look-Locker sequence (slice thickness $10 \mathrm{~mm}$, TR/TE 3.6/1.7 ms, flip angle 8, FOV $370 \mathrm{~mm}$, resolution 256 × 256, 39 phases, phase interval $15 \mathrm{~ms}$ ) was applied to determine the inversion time (TI) to optimally "null" LV myocardium (typical TI range 200-280 ms) for the subsequent scan. To evaluate the presence of myocardial late gadolinium enhancement (LGE) a breath-hold 3D inversion recovery gradient echo sequence covering the entire LV (acquired slice thickness $12 \mathrm{~mm}$, reconstructed slice thickness $6 \mathrm{~mm}$, average TR/TE 3.9/2.4 ms, multishot (50 profile/shot) segmented partial echo readout every heartbeat, flip angle 15 , field of view $400 \mathrm{~mm}$, matrix $256 \times 256$, acquired and reconstructed pixel size $1.56 \times$ $1.56 \mathrm{~mm}$, typically 16-18 slices) was used with images in short-axis, two-chamber and four-chamber view, acquired 10 minutes after the administration of intravenous contrast.

\section{CMR data analysis}

MR images were analyzed with commercially available software (CAAS MRV 3.0, Pie medical imaging, Maastricht, The Netherlands). Endocardial and epicardial contours were manually traced in end-diastolic and end-systolic phases on short axis cine images to determine end-diastolic and end-systolic volume, ejection fraction and LV enddiastolic mass. Systolic LV dysfunction was defined as an ejection fraction $<55 \%$ or regional wall motion abnormalities. LV and right ventricular (RV) dilatation were defined as enddiastolic volumes $>2 S D$ and RV systolic dysfunction as ejection fraction <2SD of mean reference values normalized for gender, body surface area and age. ${ }^{14}$ We considered LV hypertrophy as increase in the LV mass and LV wall hypertrophy as wall thickness $>12 \mathrm{~mm}$. The presence and localization of edema or focal fibrosis was visually identified by a consensus of two independent experienced observers using the T2-weighted and LGE images. CMR was considered to be abnormal if regional or global dysfunction, ventricular dilatation, hypertrophy, or areas of fibrosis or edema were observed. 


\section{Statistical analysis}

Descriptive statistics of clinical characteristics, electrocardiographic findings and cardiac magnetic resonance results are presented. Categorical variables were summarized by frequency counts (percentage) and differences between groups were evaluated using chi-square test. For continuous variables, results are presented as median (range) and comparison between categories was made with Mann-Whitney $U$ test. Multiple group comparisons were made with Kruskal-Wallis test. All analyses were performed using SPSS software version 15.0. A probability $(p)$ value of $<0.05$ at a two-sided level was considered statistically significant.

\section{Results}

\section{Patient characteristics}

Characteristics of 80 MD1 patients (all Caucasian; 45 males and 35 females) are shown in Table 1. No significant differences in clinical characteristics were found between men and women. As expected, with increasing CTG repeat length, the median age at onset and skeletal muscle strength score decreased $(p<0.001)$. All patients were ambulant for short distances (<100 meter), but 16 subjects used mobility aids and 4 patients were confined to a wheelchair for longer distances. Fatigue and dyspnoea were frequently reported symptoms: 48 patients were either dyspnoeic or fatigued after exertion and 10 subjects complained of both. No patient reported a history of syncope, severe palpitations at rest, angina pectoris or myocardial infarction. Mild peripheral edema was seen in four patients, all of whom had normal systolic and diastolic LV function and normal NT-proBNP levels.

Table 1: Clinical and genetic characteristics according to clinical phenotype category

\begin{tabular}{lllll}
\hline & Mild type & Classical type & $\begin{array}{c}\text { Congenital/ } \\
\text { childhood } \\
\text { type } \mathrm{n}=8\end{array}$ & $\mathrm{n}=80$ \\
\hline Male & $5(56 \%)$ & $33(52 \%)$ & $7(88 \%)$ & $45(56 \%)$ \\
Age in years (range) & $60(46-70)$ & $47(24-64)$ & $32(24-51)$ & $48(24-70)$ \\
Age at onset in years (range) & $52(50-65)^{*}$ & $27(10-51)$ & $6.5(0-10)$ & $27(0-65)$ \\
Muscle strength sumscore (range) & $110(107-110)$ & $96(73-109)$ & $100(78-109)$ & $98(73-110)$ \\
Abnormal ECG & $1(11 \%)$ & $41(65 \%)$ & $7(88 \%)$ & $49(61 \%)$ \\
Abnormal CMR & $3(33 \%)$ & $28(44 \%)$ & $4(50 \%)$ & $35(44 \%)$ \\
\hline
\end{tabular}

*Age at onset of neuromuscular signs and symptoms could not be determined in 3 patients, since they were still asymptomatic. 
Functional or structural abnormalities were detected with CMR in 35 patients (44\%). The results of CMR analysis are summarized in table 2. LV systolic dysfunction was found most frequently, being present in 20 patients. An example of LV dysfunction on CMR is shown in figure 1. Concomitant dilatation of the LV was found in 4 patients, while 3 patients had LV dilatation with preserved systolic function. Regional LV hypokinesia was observed in 11 patients and co-localized with local thinning of the wall in 3 cases. LV hypertrophy was observed in 6 patients. None of the patients with LV hypertrophy had arterial hypertension. LV mass indexes of MD1 patients were remarkably low and the mean values differed significantly from values obtained from healthy volunteers (t-test, $\mathrm{p}<0.001) .{ }^{14}$ Right ventricular dysfunction or dilatation was only present in patients with LV dysfunction. Abnormal myocardial function and structure was more frequent in men than in women $(p<0.001)$ and associated with higher age $(p=0.04)$, but not with duration of disease, muscle strength sumscore or CTG repeat length.

Table 2: CMR results

\begin{tabular}{|c|c|c|c|}
\hline & $\begin{array}{l}\text { All } \\
n=80\end{array}$ & $\begin{array}{l}\text { Male } \\
n=45\end{array}$ & $\begin{array}{l}\text { Female } \\
\mathrm{n}=35\end{array}$ \\
\hline LV ejection fraction, \% (range) & $58(38-73)$ & $57(45-73)$ & $61(38-71)$ \\
\hline LV systolic dysfunction, n (\%) & $20(25 \%)$ & $16(36 \%)$ & $4(11 \%)$ \\
\hline LV enddiastolic volume, $\mathrm{ml} / \mathrm{m}^{2}$ (range) & $72(38-117)$ & $77(41-117)$ & $67(38-104)$ \\
\hline LV endsystolic volume, $\mathrm{ml} / \mathrm{m}^{2}$ (range) & $31(11-63)$ & $35(14-63)$ & $28(11-56)$ \\
\hline LV dilatation, $\mathrm{n}(\%)$ & $7(9 \%)$ & $6(13 \%)$ & $1(3 \%)$ \\
\hline LV mass, $\mathrm{g} / \mathrm{m}^{2}$ (range) & $47(30-79)$ & $50(36-79)$ & $41(30-67)$ \\
\hline LV wall hypertrophy, n (\%) & $6(8 \%)$ & $4(9 \%)$ & $2(6 \%)$ \\
\hline RV ejection fraction, \% (range) & $64(38-77)$ & $60(38-77)$ & $67(50-76)$ \\
\hline RV systolic dysfunction, n (\%) & $4(5 \%)$ & $4(9 \%)$ & 0 \\
\hline RV enddiastolic volume, $\mathrm{ml} / \mathrm{m}^{2}$ (range) & $66(40-117)$ & $71(40-117)$ & $61(40-102)$ \\
\hline RV endsystolic volume, $\mathrm{ml} / \mathrm{m}^{2}$ (range) & $23(10-66)$ & $28(10-66)$ & $20(10-46)$ \\
\hline RV dilatation, n (\%) & $1(1 \%)$ & $1(2 \%)$ & 0 \\
\hline RV outflow tract, mm (range) & $26(21-37)$ & $27(22-37)$ & $25(21-29)$ \\
\hline Myocardial fibrosis, n (\%) & $10(13 \%)$ & $7(16 \%)$ & $3(9 \%)$ \\
\hline
\end{tabular}



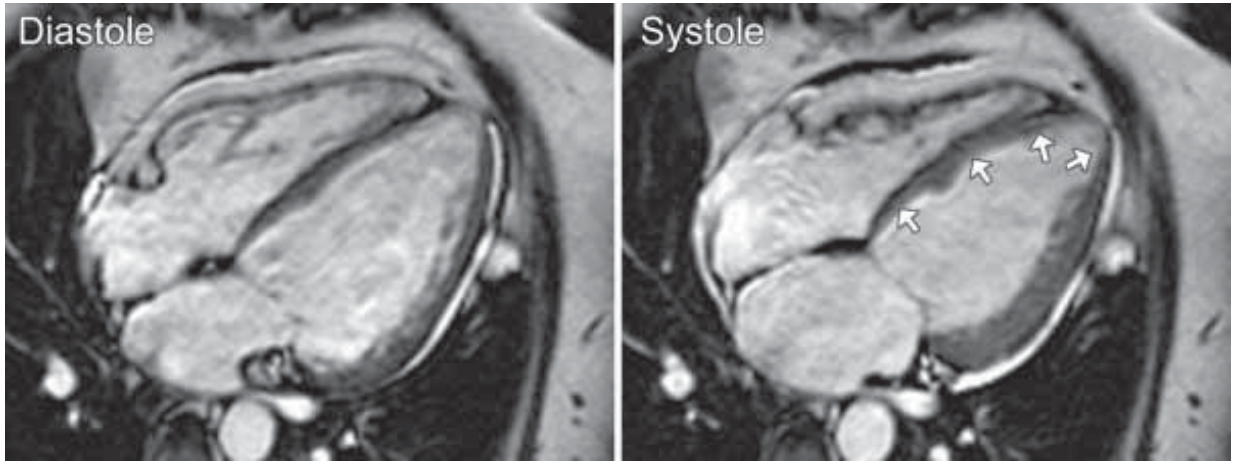

Figure 1: Ventricular dysfunction in myotonic dystrophy type 1 by CMR.

Cine images in four-chamber long-axis view in diastole and systole of a patient with impaired systolic left ventricular function (ejection fraction 38\%): septal and apical hypokinesia (arrows).
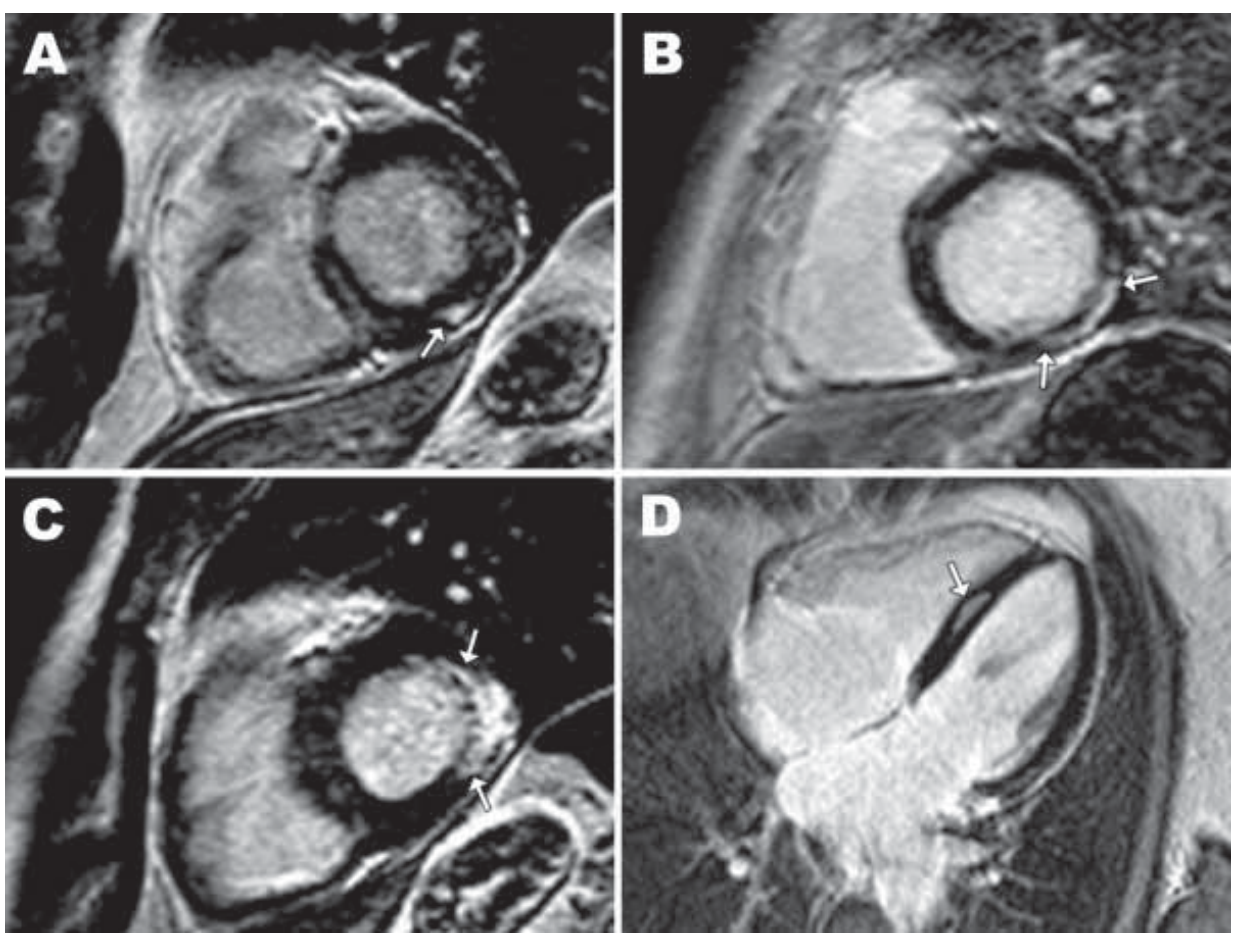

Figure 2: Myocardial fibrosis in myotonic dystrophy type 1 by CMR.

Late gadolinium enhancement (LGE) images in short axis (A, B and C) and 4-chamber long axis views (D) of 4 patients with myotonic dystrophy type 1 . Between arrows are regions of increased signal intensity, indicating focal fibrosis, visible as mid-myocardial enhancement to epicardial enhancement with endocardial sparing.

Focal myocardial fibrosis was detected on LGE images in 10 patients, most often as midmyocardial enhancement of the septal segments and basal (inferio) lateral segments of the LV wall $(n=8)$. Subendocardial and partly transmural enhancement of the 
basal lateral wall was also found $(n=2)$. Examples of selected CMR images are shown in figure 2. No patient had signs of myocardial edema on T2-weighted images. No significant relationship between the presence of myocardial fibrosis and MD1 phenotype or CTG repeat length was observed.

\section{ECG and echocardiography}

Electrocardiographic findings are summarized in table 3. An abnormal ECG was recorded in 49 patients (61\%). All patients were in sinus rhythm except for 2 with atrial fibrillation. Conduction delay was present in 46 patients (58\%) and 1 patient had abnormal Qwaves. There were no signs of hypertrophy on the ECGs. In general, patients with rhythm or conduction disturbances had more severe skeletal muscle weakness than those without $(p=0.002)$. Of the 31 patients with normal ECGs, seven showed sinusbradycardia without conduction abnormalities (frequency 50-60 bpm, $\mathrm{n}=4$; frequency $<50 \mathrm{bpm}, \mathrm{n}=3$ ).

Table 3: Electrocardiography results

\begin{tabular}{ll}
\hline & $\mathrm{n}=80$ \\
\hline Frequency, bpm (range) & $70(40-95)$ \\
Sinusbradycardia, $\mathrm{n}(\%)$ & $14(18 \%)$ \\
Atrial fibrillation, $\mathrm{n}(\%)$ & $2(3 \%)$ \\
PR interval, ms (range) & $200(136-460)$ \\
Prolonged PR interval, $\mathrm{n}(\%)$ & $30(38 \%)$ \\
QRS duration, ms (range) & $100(80-164)$ \\
Intraventricular conduction delay, $\mathrm{n}(\%)$ & $26(33 \%)$ \\
Left anterior fascicular block, $\mathrm{n}(\%)$ & $6(8 \%)$ \\
Left posterior fascicular block, $\mathrm{n}(\%)$ & $1(1 \%)$ \\
\hline
\end{tabular}

* Sinusbradycardia was defined as frequency $<60 \mathrm{bpm}$.

Echocardiography ruled out hemodynamically significant valvular disease or elevated right ventricular pressures in all patients.

A graphic reproduction of the cardiac evaluation is shown in figure 3. There was an association between ECG abnormalities and abnormal CMR findings ( $p<0.001$ ). Patients with an abnormal ECG were more likely to have functional or structural cardiac abnormalities (Odds ratio 8.2; 95\% Cl 2.7-25.1). However, myocardial involvement was also seen in 5 out of 31 patients with a normal ECG. The sensitivity of the ECG to predict myocardial involvement in this selected population was $86 \%$, with a specificity of $58 \%$. 
Late gadolinium enhancement was found in 9 out of 49 patients with ECG abnormalities and only 1 of the 31 patients with a normal ECG. NT-proBNP levels did not significantly differ between patients with and without cardiac conduction disease or myocardial abnormalities. Complaints of fatigue and dyspnoea on exertion were not associated with abnormalities on ECG or imaging.

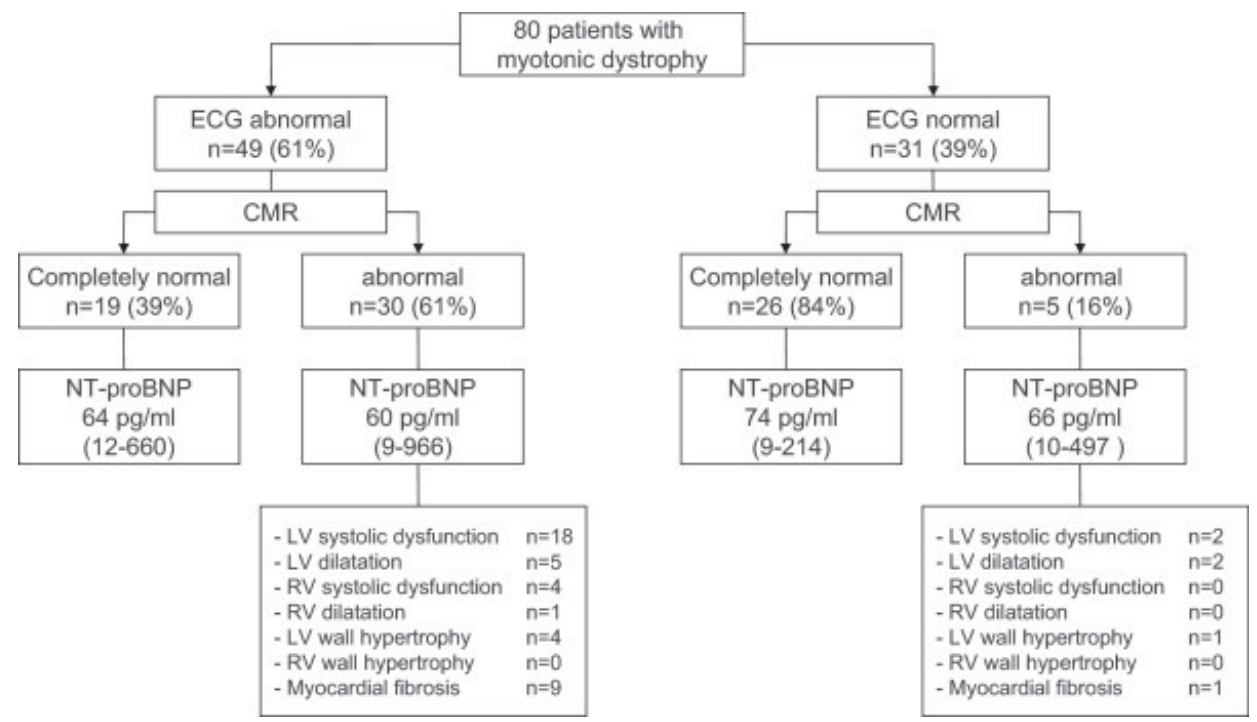

Figure 3: Graphic reproduction of cardiac evaluation in myotonic dystrophy patients showing ECG and CMR findings. The majority of patients with ECG abnormalities had functional or structural cardiac abnormalities. However, a substantial number of patients with normal ECG also showed myocardial alterations. NT-proBNP levels did not help to distinguish between patients with and without impaired myocardial functioning (presented as median (range)).

\section{Discussion}

The principal finding of this study is that structural and functional myocardial abnormalities are frequent in MD1 patients. The presence of mild to moderate left ventricular systolic dysfunction, ventricular dilatation, myocardial hypertrophy or fibrosis was strongly associated with electrocardiographic conduction abnormalities. However, 16\% of patients with a normal ECG still had myocardial alterations. These findings lend support to the concept that the myocardium is generally involved in the pathogenic process of MD1.

Myocardial involvement may be prognostic in predicting death in MD1. ${ }^{9} \mathrm{CMR}$ is well established in cardiomyopathies, because of its greater sensitivity and reproducibility than conventional diagnostic investigations (ECG and echocardiography) to demonstrate early abnormalities or subtle changes. ${ }^{15}$ However, the CMR phenotype of MD1 
had not been well characterized. Initial descriptions of CMR findings in MD1 revealed structural abnormalities, but the number of investigated subjects was small and functional analysis had not been carried out. ${ }^{16}$ Increased left ventricular trabeculation confirmed by CMR has been reported in two related patients with MD1. ${ }^{17}$ Another study described a possible relationship between CMR abnormalities of the right ventricle and inducible arrhythmias at electrophysiological testing in MD1 patients. ${ }^{18}$ Yet, no gadolinium contrast was used to visualize fibrosis and the induced ventricular arrhythmias were mostly non-sustained. We did not find any isolated remarkable abnormalities of the right ventricle or left ventricular trabeculation in our large cohort.

Fibrosis is a frequent histopathological finding in individual MD1 cases. ${ }^{19-23}$ Focal myocardial fibrosis as detected by LGE-CMR was present in $13 \%$ of our patients. As in other non-ischemic cardiomyopathies, late gadolinium enhancement was usually located in the interventricular septum and often limited to the mid-myocardium. ${ }^{24,25}$ An increased risk of sustained ventricular tachycardia and sudden death is associated with midwall fibrosis in patients with dilated cardiomyopathy. ${ }^{26}$ Whether midwall fibrosis determined by CMR is a predictor of mortality in MD1 remains to be investigated.

The low prevalence of symptomatic heart failure in MD1 is usually partly attributed to the reduced cardiac demand due to diminished skeletal muscle activity. ${ }^{27}$ This lower hemodynamic load in MD1 patients can also explain the low LV mass indexes found in this study.

Increasing age, male sex and ECG conduction abnormalities are all significantly associated with myocardial disease, whereas CTG repeat length and severity of muscular impairment are not. Male gender and age have been positively associated with arrhythmia and conduction abnormalities. ${ }^{28}$ There is no consensus from the literature as to whether or not CTG repeat size has value as a prognostic indicator of conduction disturbances or cardiac events. ${ }^{28-30}$ While age at onset of symptoms and severity of the phenotype correlate with the size of the CTG repeat, the association between the length of the CTG repeat measured in leukocytes and other symptoms of MD1 is more elusive. The heterogeneity of symptoms shown by patients with similar CTG repeat sizes can partly be explained by the presence of somatic mosaicism and somatic expansion over time. $^{31}$

Structural and functional cardiac changes were found in patients with mild as well as severe neurological phenotypes. Duration of neuromuscular disease was not significantly related to cardiac disease, indicating that cardiac manifestations can precede, coincide with or succeed skeletal myopathy. It should however be stressed that recall of age at onset is sometimes poor and unreliable, as the diagnosis is often considerably delayed. Furthermore, duration of symptoms do not necessarily relate to the severity of neuromuscular symptoms as disease progression is highly variable. Symptoms of dyspnoea or fatigue were not associated with LV dysfunction and may therefore largely be ascribed to the progressive physical disability of the muscular disease. 
The current study is a descriptive study of a large cohort of patients with MD1 using state of the art diagnostic technology. A limitation of the descriptive survey is the absence of a comparison group or prognostic data allowing no inferences to be drawn about cause of disease and the predictive value of myocardial fibrosis or other CMR findings for identifying patients with MD1 who are at risk for cardiac death.

\section{Conclusions}

Subclinical cardiomyopathy in patients with MD1 is frequently observed with CMR. Screening for functional and structural cardiac disease should be considered in all patients since myocardial involvement can be overlooked by ECG alone. Whether the identification of structural or functional cardiac changes has prognostic implications for the prediction of disease progression or sudden death remains to be investigated in a long-term prospective study.

\section{References}

1. Harper PS. Myotonic dystrophy. 3. Saunders, London; 2001.

2. Harley HG, Rundle SA, MacMillan JC, Myring J, Brook JD, Crow S, Reardon W, Fenton I, Shaw DJ, Harper PS. Size of the unstable CTG repeat sequence in relation to phenotype and parental transmission in myotonic dystrophy. Am J Hum Genet. 1993;52:1164-1174.

3. de Die-Smulders CE, Howeler CJ, Thijs C, Mirandolle JF, Anten HB, Smeets HJ, Chandler KE, Geraedts JP. Age and causes of death in adult-onset myotonic dystrophy. Brain. 1998;121:1557-1563.

4. Mathieu J, Allard P, Potvin L, Prevost C, Begin P. A 10-year study of mortality in a cohort of patients with myotonic dystrophy. Neurology. 1999;52:1658-1662

5. Lazarus A, Varin J, Ounnoughene Z, Radvanyi H, Junien C, Coste J, Laforet P, Eymard B, Becane HM, Weber $\mathrm{S}$, Duboc D. Relationships among electrophysiological findings and clinical status, heart function, and extent of DNA mutation in myotonic dystrophy. Circulation. 1999;99:1041-1046.

6. Groh WJ, Groh MR, Saha C, Kincaid JC, Simmons Z, Ciafaloni E, Pourmand R, Otten RF, Bhakta D, Nair GV. et al. Electrocardiographic abnormalities and sudden death in myotonic dystrophy type 1. N Engl J Med. 2008;358:2688-2697.

7. Cox GF, Kunkel LM. Dystrophies and heart disease. Curr Opin Cardiol. 1997;12:329-343.

8. Bushby K, Muntoni F, Bourke JP. 107th ENMC international workshop: the management of cardiac involvement in muscular dystrophy and myotonic dystrophy. 7th-9th June 2002, Naarden, the Netherlands. Neuromuscul Disord. 2003;13:166-172.

9. Bhakta D, Groh MR, Shen C, Pascuzzi RM, Groh WJ. Increased mortality with left ventricular systolic dysfunction and heart failure in adults with myotonic dystrophy type 1. Am Heart J. 2010;160:1137-1141.

10. Grothues F, Smith GC, Moon JC, Bellenger NG, Collins P, Klein HU, Pennell DJ. Comparison of interstudy reproducibility of cardiovascular magnetic resonance with two-dimensional echocardiography in normal subjects and in patients with heart failure or left ventricular hypertrophy. Am J Cardiol. 2002;90:29-34.

11. Aids to the examination of the peripheral nervous system. Her Majesty's Stationary Office, London; 1943. pp. 1-2.

12. Kleyweg RP, van der Meche FG, Schmitz PI. Interobserver agreement in the assessment of muscle strength and functional abilities in Guillain-Barre syndrome. Muscle Nerve. 1991;14:1103-1109. 
13. Mathieu J, Boivin H, Meunier D, Gaudreault M, Begin P. Assessment of a disease-specific muscular impairment rating scale in myotonic dystrophy. Neurology. 2001;56:336-340.

14. Hudsmith LE, Petersen SE, Francis JM, Robson MD, Neubauer S. Normal human left and right ventricular and left atrial dimensions using steady state free precession magnetic resonance imaging. J Cardiovasc Magn Reson. 2005;7:775-782.

15. Verhaert D, Richards K, Rafael-Fortney JA, Raman SV. Cardiac involvement in patients with muscular dystrophies: magnetic resonance imaging phenotype and genotypic considerations. Circ Cardiovasc Imaging. 2011;4:67-76.

16. De Ambroggi L, Raisaro A, Marchiano V, Radice S, Meola G. Cardiac involvement in patients with myotonic dystrophy: characteristic features of magnetic resonance imaging. Eur Heart J. 1995;16:1007-1010.

17. Finsterer J, Stollberger C, Kopsa W. Familial left ventricular hypertrabeculation in myotonic dystrophy type 1. Herz. 2003;28:466-470. doi: 10.1007/s00059-003-2437-4.

18. Vignaux O, Lazarus A, Varin J, Coste J, Carlier P, Argaud C, Laforet P, Weber S, Legmann P, Duboc D. Right ventricular MR abnormalities in myotonic dystrophy and relationship with intracardiac electrophysiologic test findings: initial results. Radiology. 2002;224:231-235.

19. Arnason G, Berge T, Dahlberg L. Myocardial Changes in Dystrophia Myotonica. Acta Med Scand. 1964;176:536-538.

20. Bulloch RT, Davis JL, Hara M. Dystrophia myotonica with heart block. A light and electron microscopic study. Arch Pathol. 1967;84:130-140.

21. Motta J, Guilleminault C, Billingham M, Barry W, Mason J. Cardiac abnormalities in myotonic dystrophy. Electrophysiologic and histopathologic studies. Am J Med. 1979;67:467-473.

22. Bharati S, Bump FT, Bauernfeind R, Lev M. Dystrophica myotonia. Correlative electrocardiographic, electrophysiologic, and conduction system study. Chest. 1984;86:444-450.

23. Nguyen HH, Wolfe JT, Holmes DR, Edwards WD. Pathology of the cardiac conduction system in myotonic dystrophy: a study of 12 cases. J Am Coll Cardiol. 1988;11:662-671.

24. McCrohon JA, Moon JC, Prasad SK, McKenna WJ, Lorenz CH, Coats AJ, Pennell DJ. Differentiation of heart failure related to dilated cardiomyopathy and coronary artery disease using gadolinium-enhanced cardiovascular magnetic resonance. Circulation. 2003;108:54-59.

25. Moon JC, McKenna WJ, McCrohon JA, Elliott PM, Smith GC, Pennell DJ. Toward clinical risk assessment in hypertrophic cardiomyopathy with gadolinium cardiovascular magnetic resonance. J Am Coll Cardiol. 2003;41:1561-1567.

26. Assomull RG, Prasad SK, Lyne J, Smith G, Burman ED, Khan M, Sheppard MN, Poole-Wilson PA, Pennell DJ. Cardiovascular magnetic resonance, fibrosis, and prognosis in dilated cardiomyopathy. J Am Coll Cardiol. 2006;48:1977-1985.

27. Phillips MF, Harper PS. Cardiac disease in myotonic dystrophy. Cardiovasc Res. 1997;33:13-22.

28. Petri H, Vissing J, Witting N, Bundgaard H, Kober L. Cardiac manifestations of myotonic dystrophy type 1. Int J Cardiol. 2012;160:82-8.

29. Sabovic M, Medica I, Logar N, Mandic E, Zidar J, Peterlin B. Relation of CTG expansion and clinical variables to electrocardiogram conduction abnormalities and sudden death in patients with myotonic dystrophy. Neuromuscul Disord. 2003;13:822-826.

30. Clarke NR, Kelion AD, Nixon J, Hilton-Jones D, Forfar JC. Does cytosine-thymine-guanine (CTG) expansion size predict cardiac events and electrocardiographic progression in myotonic dystrophy? Heart. 2001;86:411-416.

31. Martorell L, Monckton DG, Gamez J, Johnson KJ, Gich I, Lopez de Munain A, Baiget M. Progression of somatic CTG repeat length heterogeneity in the blood cells of myotonic dystrophy patients. Hum Mol Genet. 1998;7:307-312. 


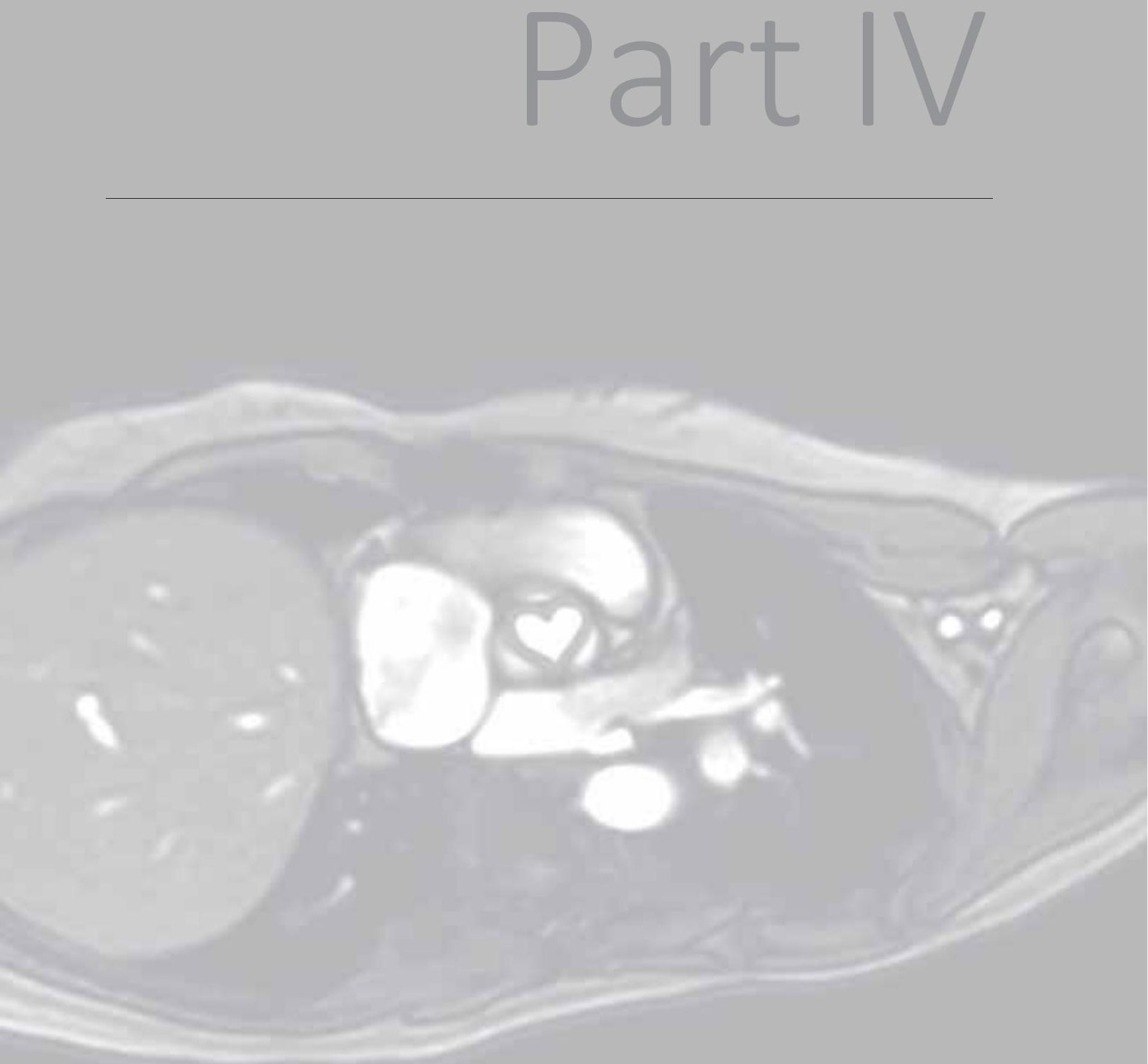

Summary, discussion and future perspectives 

Cardiovascular magnetic resonance imaging (CMR) has shown enormous growth over the past two decades and matured as a cardiac imaging modality with excellent diagnostic and prognostic performance in cardiomyopathies, ischemic and congenital heart disease. ${ }^{1,2}$ CMR is a comprehensive imaging technique for the detection and assessment of cardiovascular disease. The first part of the thesis focusses on fast cardiac MR imaging with real-time imaging techniques. Real-time cine images can already be displayed and analyzed during the process of scanning and do not require breath-holding or ECG-gating. In contrast, current cine imaging to assess ventricular function, volumes and mass is based on ECG-gated cine balanced steady state free precession sequences using parallel imaging. Short axis images, covering the entire heart, with high temporal and spatial resolution are acquired within 5 to 10 breath-holds. Each breath-hold has a duration of approximately 10s. Excellent temporal and spatial resolution are a result of acquiring data over a number of cardiac cycles requiring constancy of heart rate and cardiac position. ${ }^{3}$ However, many patients find it difficult to follow breath-hold instructions. Furthermore, 10s are too long for patients with heart failure who frequently are also not able to lay flat in the bore of the MR scanner. Patients may also have atrial fibrillation or frequent extrasystoles. Thus, real-time imaging sequences are important. Breath-holding and ECG gating are not required. K-space is usually filled in a single shot and the matrix is reduced resulting in acquisition times of less than $100 \mathrm{~ms}$ for a single image. ${ }^{4}$ Real-time imaging is currently applied 1 ) in the beginning of each cardiac MR examination during interactive scan planning. It saves time and makes the process of planning the correct heart axes easier. Image quality is less important during scan planning. 2) In certain indications such as constrictive pericarditis where free breathing is required to demonstrate septal bounce. 3) To replace standard breath-hold, ECGtriggered cine balanced steady state free precession sequences if image quality is suboptimal due to atrial fibrillation, frequent extrasystoles or the patient is not able to hold his breath or follow breath-hold instructions. 4) In interventional MR imaging. In chapters 2 and 3, real-time catheter guidance with MR imaging (MR fluoroscopy) is described in an animal model of atrial septal defect. In chapter 2, left and right heart catheterization using MR fluoroscopy for catheter steering is described and found to be feasible. A prototype active tracking catheter was used to obtain blood pressures and samples from cardiac chambers and great vessels using antegrade, transseptal, and retrograde approaches. A new method to determine vascular resistance is shown: In combination with velocity-encoded cine MR for measuring pulmonary and aortic blood flow, it is possible to calculate vascular resistances. Thus, left and right heart catheterization using real-time MR guidance is feasible and has the potential to change the current $\mathrm{x}$-ray-based diagnostic approach for children with congenital heart disease. Hemodynamic catheterization data can be combined with anatomic and functional MR imaging. This may significantly improve the evaluation of patients with complex congenital heart disease. In chapter 3 it is explained that even small left-to-right shunt volumes ( $Q p$ : Qs < 1.5) were accurately detected by MR flow imaging. Furthermore, balloon sizing of 
atrial septum defects with MR imaging was performed for the first time. This allows to measure the size of atrial septal defects even in case of small shunt volumes. Finally, transcatheter closure of atrial septal defects under MR-guidance is described in this chapter.

Many proof-of-concept and preclinical cardiac interventional MR studies have been performed in animals and humans. ${ }^{5-10}$ The introduction of MR-guided catheterization into clinical practice, however, depends on the development and availability of MRcompatible catheters and guidewires. Scanner design needs to be optimized for interventional procedures, e.g. open or smaller magnets. MR-safe guidewires are under development, but currently not available for clinical use. ${ }^{11}$ Passive MR-compatible catheters (e.g. catheters or balloons filled with contrast material) may be used for MRguided interventions. However, MR provides inferior visibility since mainly the tip is visible during an intervention. ${ }^{12}$ Active catheters (e.g. catheters embedding antennae), such as the one described in chapter 2 and 3, could be visualized faster and potentially also as a whole from shaft to tip, but are currently not approved for clinical use. Large MR scanners with small bores are suboptimal for patients and interventional physicians. Noise, communication during scanning, no true 3D image display and equipment such as monitors and defibrillators have also to be addressed. Thus, $x$-ray fluoroscopy is the mainstay for guiding interventional procedures. A wide range of different catheters and guidewires is available. X-ray fluoroscopy allows constant visibility of the whole catheter and offers superior spatial as well as temporal resolution. Cardiac interventional MR is currently only considered when anatomical details are important and tissue characterization is needed, such as in congenital heart disease as well as in electrophysiological studies. ${ }^{13-15}$ Regarding electrophysiological interventions, MR imaging and catheter guidance are of great importance. Atria are complex structures with large anatomical variability. In the left ventricle, definition of target regions for ablation of ventricular tachycardia, e.g. regions of infarct, is required. Furthermore, it is not enough to precisely know where the catheter tip is, but also to image what the tip has done in terms of ablation lesions. ${ }^{16}$ Therefore, MR imaging and -fluoroscopy is promising for electrophysiological studies. ${ }^{17}$

A real-time flow measurement technique to evaluate peak velocity and flow volume in different parts of the aorta, carotid and iliacal arteries is presented in Chapter 4. ECGtriggering was not necessary and scan time reduced from 2 minutes to 6 seconds. In combination with interactive scan planning, the real-time flow measurement technique allows adaptation of the imaging plane during scanning. Peak velocity correlated in all examined vessels with standard flow measurements, while volume flow correlated in the largest vessels only. Evaluation of flow during physiologic maneuvers or during pharmacological stress is possible using real-time flow sequences. ${ }^{18}$ They also allow rapid assessment of changes in cardiac output or simultaneous measurements of mitral and tricuspid flow e.g. in patients with constrictive pericarditis to demonstrate discordant respirophasic changes in atrioventricular valve inflow velocities. ${ }^{19}$ Fast imaging of 
flow might allow to incorporate hemodynamic data into standard imaging, comparable to Doppler-imaging in echocardiography. Current research is focused on improvements in spatial and temporal resolution as well as fast image analysis already during scanning. $^{20,21}$

In chapter 5 a real-time cine imaging technique is described and compared with a standard breath-hold imaging sequence during dobutamine stress. Image quality was comparable to standard imaging for images in short axis view, but not for other imaging planes. The breath-hold imaging sequence used in this publication is now replaced by balanced steady state free precession sequences since they show improved border delineation. ${ }^{22}$ Thus, many efforts have taken place to introduce newer real-time sequences to compete with image quality of state-of-the-art balanced steady state free precession sequences. ${ }^{3,23-25}$ A recent real-time imaging strategy was to apply radial kspace sampling and image reconstruction by gridding to balanced steady state free precession sequences: Left ventricular functional analysis by real-time cine imaging with a balanced steady state free precession sequence and radial k-space sampling, gridding and sliding window reconstruction, with a nominal temporal resolution of 40 frames per second was in good agreement with the standard ECG-gated breath-hold balanced steady state free precession sequence. ${ }^{26}$ Similar results were recently published: Image quality of the real-time scans was rated as diagnostic and functional parameters were not different from standard imaging. ${ }^{27}$ Real-time imaging at 3 Tesla, where standard balanced steady state free precession sequences are sensitive to local magnetic field inhomogeneities and susceptibility effects at high filed strength, ${ }^{26}$ has also been introduced. $^{28,29}$

In conclusion, real-time imaging is widely employed now for interactive scan planning, but not as the main imaging sequences due to image quality. However, there are currently many ongoing efforts to replace or improve standard cine imaging in order to increase patient comfort and compliance by shortening the scan duration, skipping ECG-gating as well as breath-holds and displaying images immediately. It cannot be accepted that, in contrast to echocardiography, scan duration is long and exhausting for patients in the field of cardiac MR imaging. X-ray fluoroscopy will currently remain the standard imaging technique for coronary and valve interventions. However, MR-guided electrophysiological interventions are just around the corner. ${ }^{13}$

In the second part of the thesis a comprehensive view on CMR imaging of ischemic heart disease is presented. Chapters 6 to 8 focus on the detection and sizing of reperfused myocardial infarction by CMR with a necrosis-specific MR contrast agent in an animal model of normal and hypertrophied left ventricle. Hypertrophied hearts showed larger infarctions than control while the areas at risk were the same. During infarction acutely administered potassium channel opener reduced infarction size to that of control. Chronic oral administration of a potassium channel opener prevented the formation of hypertrophy, and infarct size was also the same as in the control group. CMR findings correlated perfectly with histology. CMR in myocardial infarction is considered 
the gold standard to assess left ventricular global and regional function, infarct size and transmurality, microvascular obstruction, hemorrhage, edema and also RV involvement and-function, and CMR-findings have prognostic implications. ${ }^{30}$ Thus, CMR is important for diagnosis and prognosis, but also for research in myocardial infarction and ischemia regarding infarct quantification and evaluation of new treatment strategies.

The current strategy to minimize the impact of acute myocardial infarction is to revascularize as early as possible. However, this strategy may have come to a preliminary end: Even earlier administration of antiplatelet therapy and revascularization did not further improve outcome or reduce infarct size, and CMR was essential in corroborating these findings. E.g. in ST-elevation myocardial infarction, prehospital antiplatelet therapy was not superior to administration in the catheter lab or emergency room, and earlier than 90 minute after begin of infarction opening of the infarct related coronary artery seemed not to further improve the outcome. ${ }^{31-33}$ In patients with non-STelevation myocardial infarction, immediate invasive revascularization did not improve outcome in comparison with early invasive strategies unless the patient had been at high risk or in cardiogenic shock. ${ }^{34-37}$ Early administration of antiplatelet medication, however, is very important in non-ST-elevation myocardial infarction. ${ }^{38}$ Antiplatelet therapy, low molecular weight heparins, beta-blockers, ACE-inhibitors and statins improve outcome. In contrast, postconditioning strategies with drugs or interventions have little impact on infarct size or outcome. ${ }^{39,40}$ Thus, new pre- and postconditioning strategies are still urgently needed to further improve myocardial function and patient outcome, and CMR will play an important role to evaluate new strategies since many concepts seemed to work in animal models, but not in humans. CMR is superior to other methods such as measuring the peak myocardial enzyme release or echocardiography. Infarct size can roughly be estimated by peak myocardial enzyme release. However, the peak can easily be missed, and other important factors such as remodeling, dilation and infarct transmurality are not addressed. Echocardiography is used to calculate the number of ventricular segments with regional wall motion abnormalities and measure left ventricular ejection fraction. Ejection fraction is often considered the most important prognostic factor after ST-elevation myocardial infarction. It correlates with infarct size, but the relation is not direct. ${ }^{41}$ Infarct size and transmurality are frequently more important. This is explained by the following example: Patients with left ventricular hypertrophy often show a supra-normal left ventricular ejection fraction. ${ }^{42-44}$ After ST-elevation myocardial infarct, they exhibit larger infarct size and transmurality than patients without hypertrophy while ejection fraction is the same. ${ }^{41}$ More pronounced hypercontractility in remote segments and a presumably higher ejection fraction before infarction in patients with hypertrophy are possible explanations. By using CMR, infarctions are reliably detected and infarct size and transmurality can accurately be measured. This is also supported by the findings presented in chapter 9. The chapter compares CMR (late gadolinium enhancement) with echocardiography (regional wall motion analysis) to detect acute and chronic myocardial infarction. Although echocardiography 
is frequently used as a first line imaging modality, its accuracy to detect or rule out acute and chronic myocardial infarction is not well described. Using echocardiography, a substantial number of acute and chronic infarctions were not detectable, especially smaller and non-transmural infarctions.

MR stress perfusion imaging for the detection of myocardial ischemia is currently performed with a high diagnostic accuracy. ${ }^{45}$ Ischemia and angina are usually a consequence of a significant stenosis of epicardial coronary arteries. However, ischemia can also be caused by vasospasm and, presumably, microvascular disease in a substantial number of patients. The prognosis of patients with ischemia but without stenosis has initially been considered comparable to healthy subjects, but it seems to be more comparable to patients with significant coronary artery stenosis. ${ }^{46,47}$ In chapter 10 data on microvascular disease causing myocardial ischemia is presented. Patients with cardiac syndrome $X$ having angina on exertion with concomitant ST-segment depressions during exercise ECG testing, but without significant coronary artery stenosis, were included in our study. During adenosine stress MR perfusion imaging, these patients showed a diminished subendocardial perfusion reserve in comparison with the subepicardial perfusion reserve. They also had a disturbed glycocalyx responsiveness as measured with noninvasive sublingual microscopy using a handheld sidestream darkfield microscan videomicroscope. Thus, endothelial glycocalyx might play a crucial role in regulating microvascular volume for myocardial perfusion. CMR perfusion imaging may help to identify patients with microvascular disease. It is important to better understand microvascular regulation since patients with cardiac syndrome $X$ frequently have persisting symptoms with impaired quality of life, and effective therapeutic strategies are currently not known. ${ }^{48-50}$

The third part is focused on non-ischemic heart disease. In chapter 11 we set out to assess focal (replacement) and interstitial (reactive) myocardial fibrosis in patients with dilated cardiomyopathy. Interstitial fibrosis was determined in endocardial biopsy specimens and focal fibrosis with late gadolinium enhancement MR imaging. The number of studies assessing focal myocardial fibrosis by late gadolinium enhancement MR in cardiomyopathies has increased over the last 10 years $^{51}$ and focal myocardial fibrosis is associated with adverse events in non-ischemic cardiomyopathy. ${ }^{52}$ However, the underlying cause of focal fibrosis is not known. Empirically, certain late enhancement patterns of focal fibrosis were considered pathognomonic such as septal midwall fibrosis for dilated cardiomyopathy. ${ }^{53,54}$ One of our hypotheses was that a certain amount of interstitial fibrosis measured histological as collagen volume fraction is necessary before focal fibrosis becomes detectable with late gadolinium MR imaging. However, there was no correlation between focal and interstitial fibrosis. In contrast, we found that focal fibrosis was related to inflammation. This correlation was not present for interstitial fibrosis.

MR imaging is the method of choice for the assessment of right ventricular function since it is difficult to measure with other imaging modalities. Right ventricular dysfunc- 
tion is correlated to limited exercise capacity and poor outcome, but often neglected in daily clinical practice. ${ }^{55-57}$ In chapter 12 the right ventricular function was measured in patients with dilated cardiomyopathy and ischemic heart disease. Right ventricular function and size were influenced by systolic and diastolic left ventricular function and pulmonary artery pressure. The right ventricular function was more impaired in dilated cardiomyopathy than in ischemic heart disease.

Due to the accuracy e.g. in the assesment of left and right ventricular volumes, ejection fraction, mass, infarction and fibrosis, CMR can be employed to detect cardiac disease in small groups of patients. Chapter 13 is a description of CMR findings in patients with myotonic dystrophy type I. Patients with this rare neuromuscular disease may develop conduction alterations, cardiomyopathy and sudden cardiac death. CMR detected abnormal findings in $44 \%$ of the population. A normal ECG did not exclude myocardial alterations.

In conclusion, CMR has become an established imaging modality for the evaluation of patients with cardiac disease. At the Maastricht University Medical center approximately 12000 echocardiographic and 600 CMR studies, 400 SPECT and 1000 coronary CT scans are annually performed. Although large differences between cardiovascular centers exist, CMR is performed in a substantial number of patients. In general, however, it is not the most often ordered cardiac imaging test due to limited availability and expertise, long imaging duration per patient and high costs. While CMR is reproducible and reliable and the only comprehensive cardiac imaging technique for function, anatomy, perfusion, infarct and scar characterization and, with limitations, coronary imaging, it is hampered by the above stated drawbacks. CMR is still a relatively new technique. Since the early days of cardiac imaging with MR, many aspects of cardiac MR imaging improved significantly. However, there are several issues that need to be addressed in the future:

1) The need for speed: The scanning needs to be faster and more often in real-time. Imaging and analysis have already become faster with the introduction of parallel imaging and digital coils, faster reconstruction and dedicated cardiac image analysis programs. However, more scan sequences were added to daily imaging protocols such as T1 and T2 mapping sequences. Real-time imaging can help to shorten scan duration. Other strategies are also needed since scan duration is important for patient comfort and costs.

2) The need for improved scanner design. Why do patients often report of discomfort when considering an MR imaging study ${ }^{58,59}$ Many patients feel that a CMR study had been more exhausting than an invasive coronary angiography although patients and physicians do not easily realize that, besides the radiation, it is an invasive procedure and carries the risk of serious adverse events. A CMR study comes with a long examination duration in a narrow bore, massive magnetic shielding including heavy doors, noise of the gradients and the fact that personnel is usually outside the scanning room. Short bore magnets and open scanners would improve patient comfort since an 
anxious patient will not easily lay still and follow breathing instructions. Open scanner designs with low magnetic fields have been developed, but they are considered inferior for perfusion and late enhancement imaging. ${ }^{60}$ In addition, the trend to higher field strengths requires larger magnets and, thus, even larger scanners. Until now, vendors and users follow the one-size-fits-all strategy because of lower costs: A huge scanner with a narrow bore is used for imaging all parts of the human body. If following this strategy, vendors need, however, to improve scanner design: more open, more space, smaller magnets, larger bores and, thus, less claustrophobia. A smaller scanner with less noise, faster acquisition and reconstruction should be the goal.

3) The need to learn from others. E.g. most MR studies on coronary artery imaging were performed without standard usage of beta-blockers and nitrates while CT and invasive angiography studies were almost always performed with premedication. It is also important to share knowledge about scan sequences with others, standardize scan protocols and keep the need for extensively adjusting scan parameters in every single patient as minimal as possible.

4) The need to make better use of techniques that are already available. 3D image acquisition and reconstruction with CMR is possible and has already been introduced. However, image analysis is usually performed with 2D image planes. Meanwhile, in the field of echocardiography, 3D imaging has become state-of-the art for certain indications such as preoperative assessment of mitral valve regurgitation. 3D computer games and wearable data glasses are around and will influence medical imaging and image analysis. Diastolic function is usually not assessed with CMR while excellent techniques such as particle tracking, tagging, mitral and pulmonary venous flow imaging and measuring atrial volumes are available, but analysis is time consuming. Currently, none of the cardiac imaging techniques offers a comprehensive cardiac examination. CMR has the potential to cover all aspects of cardiovascular imaging, but needs to become more competitive in coronary artery imaging.

5) The need to more extensively demonstrate that CMR is important for diagnosis, therapy and prognosis of cardiac patients. Imaging plays a crucial role in the diagnostic and therapeutic process of cardiac patients and is increasingly performed despite limited financial resources. Physicians even use hand-held echocardiography devices in order to visualize and remember the specific cardiac conditions of a certain patient. Research assessing cost effectiveness, diagnostic superiority and improved outcome after the use of cardiac imaging is sparse, but growing. ${ }^{1,2,61}$ Publications on CMR mostly addressed single prognostic markers such as fibrosis in DCM, HCM and aortic stenosis; infarct size, transmurality, microvascular obstruction, hemorrhage in patients with acute myocardial infarction; ejection fraction, LV size and geometry. However, knowledge of an adverse prognosis does not automatically improve therapy. ${ }^{2}$ In addition, direct comparisons between imaging modalities are also sparse. ${ }^{62-65}$ Clinical trials assessing the diagnostic performance of different cardiac imaging techniques for stable angina or for revascularization are currently ongoing. ${ }^{61,66,67}$ 
Thus, there are five great challenges for cardiovascular magnetic resonance imaging and, by far, more opportunities: The future of CMR is bright. Let us start working.

\section{References}

1. Flett AS, Westwood MA, Davies LC, et al. The prognostic implications of cardiovascular magnetic resonance. Circ Cardiovasc Imaging. 2009;2:243-250.

2. Francis SA, Daly C, Heydari B, et al. Cost-effectiveness analysis for imaging techniques with a focus on cardiovascular magnetic resonance. J Cardiovasc Magn Reson. 2013;15:52.

3. La Gerche A, Claessen G, Van de Bruaene A, et al. Cardiac MRI: a new gold standard for ventricular volume quantification during high-intensity exercise. Circ Cardiovasc Imaging. 2013;6:329-338.

4. Bogaert J, Dymarkowski S, Taylor A. Clinical cardiac MRI. Springer Berlin Heidelberg New York 2005

5. Schalla S, Saeed M, Higgins CB, et al. Magnetic resonance--guided cardiac catheterization in a swine model of atrial septal defect. Circulation. 2003;108:1865-1870.

6. Schalla S, Saeed M, Higgins CB, et al. Balloon sizing and transcatheter closure of acute atrial septal defects guided by magnetic resonance fluoroscopy: assessment and validation in a large animal model. J Magn Reson Imaging. 2005;21:204-211.

7. Piorkowski C, Grothoff M, Gaspar T, et al. Cavotricuspid isthmus ablation guided by real-time magnetic resonance imaging. Circ Arrhythm Electrophysiol. 2013;6:e7-10.

8. Horvath KA, Guttman M, Li M, et al. Beating heart aortic valve replacement using real-time MRI guidance. Innovations (Phila). 2007;2:51-55.

9. Kuehne T, Yilmaz S, Schulze-Neick I, et al. Magnetic resonance imaging guided catheterisation for assessment of pulmonary vascular resistance: in vivo validation and clinical application in patients with pulmonary hypertension. Heart. 2005;91:1064-1069.

10. Tzifa A, Krombach GA, Kramer N, et al. Magnetic resonance-guided cardiac interventions using magnetic resonance-compatible devices: a preclinical study and first-in-man congenital interventions. Circ Cardiovasc Interv. 2010;3:585-592.

11. Wolska-Krawczyk M, Rube MA, Immel E, et al. Heating and safety of a new MR-compatible guidewire prototype versus a standard nitinol guidewire. Radiol Phys Technol. 2014;7:95-101.

12. Ratnayaka K, Faranesh AZ, Hansen MS, et al. Real-time MRI-guided right heart catheterization in adults using passive catheters. Eur Heart J. 2013;34:380-389.

13. Grothoff M, Piorkowski C, Eitel C, et al. MR imaging-guided electrophysiological ablation studies in humans with passive catheter tracking: initial results. Radiology. 2014;271:695-702.

14. Schmitt B, Steendijk P, Ovroutski S, et al. Pulmonary vascular resistance, collateral flow, and ventricular function in patients with a Fontan circulation at rest and during dobutamine stress. Circ Cardiovasc Imaging. 2010;3:623-631.

15. White MJ, Thornton JS, Hawkes DJ, et al. Design, Operation, and Safety of Single-Room Interventional MRI Suites: Practical Experience From Two Centers. J Magn Reson Imaging 2014;4:24577.

16. Segerson N, Lim T, Marrouche NF. Towards optimization of imaging for ablation of atrial fibrillation: the search for a gold standard. Heart Rhythm. 2008;5:965-967.

17. Koopmann M, Marrouche NF. Why hesitate introducing real-time magnetic resonance imaging into the electrophysiological labs? Europace. 2013;15:7-8.

18. Fasshauer M, Joseph AA, Kowallick JT, et al. Real-time phase-contrast flow MRI of haemodynamic changes in the ascending aorta and superior vena cava during Mueller manoeuvre. Clin Radiol. 2014;69:10661071.

19. Thavendiranathan $P$, Verhaert $D$, Walls $M C$, et al. Simultaneous right and left heart real-time, freebreathing CMR flow quantification identifies constrictive physiology. JACC Cardiovasc Imaging. 2012;5:1524. 
20. Kowalik GT, Steeden JA, Pandya B, et al. Real-time flow with fast GPU reconstruction for continuous assessment of cardiac output. J Magn Reson Imaging. 2012;36:1477-1482.

21. Joseph A, Kowallick JT, Merboldt KD, et al. Real-time flow MRI of the aorta at a resolution of 40 msec. J Magn Reson Imaging. 2014;40:206-213.

22. Thiele H, Nagel E, Paetsch I, et al. Functional cardiac MR imaging with steady-state free precession (SSFP) significantly improves endocardial border delineation without contrast agents. J Magn Reson Imaging. 2001;14:362-367.

23. Tsao J, Kozerke $S$, Boesiger $P$, et al. Optimizing spatiotemporal sampling for k-t BLAST and k-t SENSE: application to high-resolution real-time cardiac steady-state free precession. Magn Reson Med. 2005; 53:1372-1382.

24. Seiberlich N, Ehses P, Duerk J, et al. Improved radial GRAPPA calibration for real-time free-breathing cardiac imaging. Magn Reson Med. 2011;65:492-505.

25. Feng L, Srichai MB, Lim RP, et al. Highly accelerated real-time cardiac cine MRI using k-t SPARSE-SENSE. Magn Reson Med. 2013;70:64-74.

26. Bauer RW, Radtke I, Block KT, et al. True real-time cardiac MRI in free breathing without ECG synchronization using a novel sequence with radial k-space sampling and balanced SSFP contrast mode. Int J Cardiovasc Imaging. 2013;29:1059-1067.

27. Aandal G, Nadig $V$, Yeh $V$, et al. Evaluation of left ventricular ejection fraction using through-time radial GRAPPA. J Cardiovasc Magn Reson. 2014;16:79.

28. Zhang S, Block KT, Frahm J. Magnetic resonance imaging in real-time: advances using radial FLASH. J Magn Reson Imaging. 2010;31:101-109.

29. Schwab F, Schwarz F, Dietrich O, et al. Free breathing real-time cardiac cine imaging with improved spatial resolution at 3 T. Invest Radiol. 2013;48:158-166.

30. El Aidi H, Adams A, Moons KG, et al. Cardiac magnetic resonance imaging findings and the risk of cardiovascular events in patients with recent myocardial infarction or suspected or known coronary artery disease: a systematic review of prognostic studies. J Am Coll Cardiol. 2014;63:1031-1045.

31. Menees DS, Peterson ED, Wang Y, et al. Door-to-balloon time and mortality among patients undergoing primary $\mathrm{PCl}$. N Engl J Med. 2013;369:901-909.

32. Wilson BH, Humphrey AD, Cedarholm JC, et al. Achieving sustainable first door-to-balloon times of 90 minutes for regional transfer ST-segment elevation myocardial infarction. JACC Cardiovasc Interv. 2013;6:1064-1071.

33. Montalescot G, van 't Hof AW, Lapostolle F, et al. Prehospital ticagrelor in ST-segment elevation myocardial infarction. N Engl J Med. 2014;371:1016-1027.

34. Windecker S, Kolh P, Alfonso F, et al. 2014 ESC/EACTS Guidelines on myocardial revascularization: The Task Force on Myocardial Revascularization of the European Society of Cardiology (ESC) and the European Association for Cardio-Thoracic Surgery (EACTS). Developed with the special contribution of the European Association of Percutaneous Cardiovascular Interventions (EAPCI). Eur Heart J. 2014;35:2541-2619.

35. Katritsis DG, Siontis GC, Kastrati A, et al. Optimal timing of coronary angiography and potential intervention in non-ST-elevation acute coronary syndromes. Eur Heart J. 2011;32:32-40.

36. Mehta SR, Granger CB, Boden WE, et al. Early versus delayed invasive intervention in acute coronary syndromes. N Engl J Med. 2009;360:2165-2175.

37. Navarese EP, Gurbel PA, Andreotti F, et al. Optimal timing of coronary invasive strategy in non-STsegment elevation acute coronary syndromes: a systematic review and meta-analysis. Ann Intern Med. 2013;158:261-270

38. Hamm CW, Bassand JP, Agewall S, et al. ESC Guidelines for the management of acute coronary syndromes in patients presenting without persistent ST-segment elevation: The Task Force for the management of acute coronary syndromes (ACS) in patients presenting without persistent ST-segment elevation of the European Society of Cardiology (ESC). Eur Heart J. 2011;32:2999-3054.

39. Waltenberger J, Gelissen M, Bekkers SC, et al. Clinical pacing post-conditioning during revascularization after AMI. JACC Cardiovasc Imaging. 2014;7:620-626. 
40. Abdelnoor M, Sandven I, Limalanathan S, et al. Postconditioning in ST-elevation myocardial infarction: a systematic review, critical appraisal, and meta-analysis of randomized clinical trials. Vasc Health Risk Manag. 2014;10:477-491.

41. Malek LA, Spiewak M, Klopotowski M, et al. Influence of left ventricular hypertrophy on infarct size and left ventricular ejection fraction in ST-elevation myocardial infarction. Eur J Radiol. 2012;81:e177-181.

42. Blake J, Devereux RB, Herrold EM, et al. Relation of concentric left ventricular hypertrophy and extracardiac target organ damage to supranormal left ventricular performance in established essential hypertension. Am J Cardiol. 1988;62:246-252.

43. Hartford M, Wikstrand JC, Wallentin I, et al. Left ventricular wall stress and systolic function in untreated primary hypertension. Hypertension. 1985;7:97-104.

44. Zabalgoitia M. Left ventricular mass and function in primary hypertension. Am J Hypertens. 1996;9:55s59s.

45. Jaarsma C, Leiner T, Bekkers SC, et al. Diagnostic performance of noninvasive myocardial perfusion imaging using single-photon emission computed tomography, cardiac magnetic resonance, and positron emission tomography imaging for the detection of obstructive coronary artery disease: a meta-analysis. J Am Coll Cardiol. 2012;59:1719-1728.

46. Sharaf B, Wood T, Shaw L, et al. Adverse outcomes among women presenting with signs and symptoms of ischemia and no obstructive coronary artery disease: findings from the National Heart, Lung, and Blood Institute-sponsored Women's Ischemia Syndrome Evaluation (WISE) angiographic core laboratory. Am Heart J. 2013;166:134-141.

47. Jespersen L, Hvelplund A, Abildstrom SZ, et al. Stable angina pectoris with no obstructive coronary artery disease is associated with increased risks of major adverse cardiovascular events. Eur Heart J. 2012;33:734-744.

48. Crea F, Lanza GA. Angina pectoris and normal coronary arteries: cardiac syndrome X. Heart. 2004;90:457463.

49. Lanza GA. Cardiac syndrome X: a critical overview and future perspectives. Heart. 2007;93:159-166.

50. Mehta PK, Goykhman P, Thomson LE, et al. Ranolazine improves angina in women with evidence of myocardial ischemia but no obstructive coronary artery disease. JACC Cardiovasc Imaging. 2011;4:514522.

51. Mewton N, Liu CY, Croisille P, et al. Assessment of myocardial fibrosis with cardiovascular magnetic resonance. J Am Coll Cardiol. 2011;57:891-903.

52. Assomull RG, Prasad SK, Lyne J, et al. Cardiovascular magnetic resonance, fibrosis, and prognosis in dilated cardiomyopathy. J Am Coll Cardiol. 2006;48:1977-1985.

53. Shah. In: Edelman RR et al.: Clinical magnetic resonance imaging. 3rd ed. New York: Elsevier press; 2005

54. Mahrholdt H, Wagner A, Judd RM, et al. Delayed enhancement cardiovascular magnetic resonance assessment of non-ischaemic cardiomyopathies. Eur Heart J. 2005;26:1461-1474.

55. de Groote P, Millaire A, Foucher-Hossein C, et al. Right ventricular ejection fraction is an independent predictor of survival in patients with moderate heart failure. J Am Coll Cardiol 1998;32:948-954.

56. Di Salvo TG, Mathier M, Semigran MJ, et al. Preserved right ventricular ejection fraction predicts exercise capacity and survival in advanced heart failure. J Am Coll Cardiol 1995;25:1143-1153.

57. Jefferies JL, Towbin JA. Dilated cardiomyopathy. Lancet 2010;375:752-762.

58. Dewey M, Schink T, Dewey CF. Claustrophobia during magnetic resonance imaging: cohort study in over 55,000 patients. J Magn Reson Imaging. 2007;26:1322-1327.

59. Berg WA, Blume JD, Adams AM, et al. Reasons women at elevated risk of breast cancer refuse breast MR imaging screening: ACRIN 6666. Radiology. 2010;254:79-87.

60. Klein HM, Meyners W, Neeb B, et al. Cardiac magnetic resonance imaging using an open 0.35 T system. J Comput Assist Tomogr. 2007;31:430-434.

61. Smulders MW, Kietselaer BL, Das M, et al. The role of cardiovascular magnetic resonance imaging and computed tomography angiography in suspected non-ST-elevation myocardial infarction patients: design and rationale of the CARdiovascular Magnetic rEsoNance imaging and computed Tomography Angiography (CARMENTA) trial. Am Heart J. 2013;166:968-975. 
62. Nagel E, Lehmkuhl HB, Bocksch W, et al. Noninvasive diagnosis of ischemia-induced wall motion abnormalities with the use of high-dose dobutamine stress MRI: comparison with dobutamine stress echocardiography. Circulation. 1999;99:763-770.

63. Greenwood JP, Maredia N, Younger JF, et al. Cardiovascular magnetic resonance and single-photon emission computed tomography for diagnosis of coronary heart disease (CE-MARC): a prospective trial. Lancet. 2012;379:453-460.

64. Schwitter J, Wacker CM, van Rossum AC, et al. MR-IMPACT: comparison of perfusion-cardiac magnetic resonance with single-photon emission computed tomography for the detection of coronary artery disease in a multicentre, multivendor, randomized trial. Eur Heart J. 2008;29:480-489.

65. Schwitter J, Wacker CM, Wilke N, et al. MR-IMPACT II: Magnetic Resonance Imaging for Myocardial Perfusion Assessment in Coronary artery disease Trial: perfusion-cardiac magnetic resonance vs. singlephoton emission computed tomography for the detection of coronary artery disease: a comparative multicentre, multivendor trial. Eur Heart J. 2013;34:775-781.

66. Rademakers F, Engvall J, Edvardsen T, et al. Determining optimal noninvasive parameters for the prediction of left ventricular remodeling in chronic ischemic patients. Scand Cardiovasc J. 2013;47:329-334.

67. Songco AV, Brener SJ. Initial strategy of revascularization versus optimal medical therapy for improving outcomes in ischemic heart disease: a review of the literature. Curr Cardiol Rep. 2012;14:397-407. 



\section{Samenvatting}



Cardiovasculaire magnetische resonantie (CMR) is een beeldvormende techniek voor de diagnostiek van hartlijden. Het is een nog relatief jonge techniek. Pas in het jaar 1977 werd voor de eerste keer een doorsnede van een borstkas afgebeeld. De eerste plaatjes van het hart die van voldoende kwaliteit waren, zijn in 1984 gepubliceerd. CMR heeft zich sindsdien tot een in de dagelijkse praktijk toegepaste techniek voor beeldvorming van hart en vaten ontwikkeld met uitstekende diagnostische waarde bij patiënten met ischemische hartziekten (zoals hartinfarcten en zuurstoftekort), cardiomyopathieën (ziekten van het hart zonder goed bekende oorzaak) en aangeboren hartafwijkingen.

Dit proefschrift beschrijft de verschillende aspecten van beeldvorming met magnetische resonantie van hartafwijkingen. Cardiale beeldvorming met echocardiografie, nucleaire technieken, computertomografie, CMR en hartkatheterisaties met röntgendoorlichting wordt vaak toegepast. In Nederland zijn in het jaar 201220.555 mensen aan hartlijden overleden en er waren 202.945 ziekenhuisopnames. Meer dan 1 miljoen mensen lijden aan cardiovasculaire aandoeningen. De hoeveelheid aan beeldvormingsonderzoeken van het hart op jaarbasis is niet bekend. Als maar bij de helft van de in het jaar 2012 opgenomen hartpatiënten beeldvorming verricht zou zijn, zijn dat al 100.000 beeldvormingsonderzoeken. CMR is niet de frequentst aangevraagde techniek binnen de beeldvormingsmodaliteiten van het hart gezien beperkte beschikbaarheid, vereiste expertise en kosten. Het is echter de enige methode die alle aspecten van cardiale beeldvorming omvat, zoals bepaling van functie, volumina en massa, bloeddoorstroming van de hartspier, detectie, groottemeting en karakterisering van hartinfarcten, littekenvorming, bloeding en water- of ijzerophoping en (met beperkingen) afbeelding van de kransslagaders. Het is een veilige onderzoeksmethode omdat er geen gebruik van röntgenstraling gemaakt wordt en ook de MR-contrast vloeistoffen zijn veilig mits ze niet bij patiënten in het eindstadium van nierfunctiestoornissen toegediend worden.

De doelstelling van dit proefschrift was de waarde van CMR voor het opsporen van hartlijden aan te tonen. Het proefschrift is hiervoor in 3 delen onderverdeeld.

Het eerste deel is gefocust op snelle CMR beeldvormingstechnieken in real-time. Dit betekent dat tijdens het maken van een bewegend plaatje zo snel mogelijk alle details erop te zien zijn zonder dat ECG-triggering of adem vasthouden nodig is. Dit is anders dan bij de standaard cine CMR sequenties waar de beelden pas achteraf berekend worden uit data van een aantal verschillende hartcycli met behulp van ECG-gating en gekoppeld aan vele ademcommando's. In tegenstelling tot real-time beeldvorming ziet men dus bij de standaard sequenties ook pas achteraf of de beelden van goede kwaliteit zijn en of de beelden ook daadwerkelijk datgene tonen wat men van plan was om af te beelden. Real-time cine scan sequenties hebben ten opzichte van standaard sequenties vaak een lagere beeldkwaliteit. Hiertegenover staan tijdwinst, de mogelijkheid om ook bij een onregelmatige hartslag goede beelden te verkrijgen en meer comfort voor de patiënt. De toepassingen van real-time sequenties worden in het eerste deel van dit proefschrift beschreven. In het begin worden twee hoofdstukken over het sturen van katheters (hartkatheterisatie) met MR-doorlichting in real-time in een diermodel met de 
aangeboren hartafwijking van atriale septumdefecten (ASD) gepresenteerd (hoofdstukken twee en drie).

In hoofdstuk twee wordt een studie getoond waarin voor de eerste keer de linker en rechter harthelft invasief met een katheter onder MR-doorlichting (het sturen van de katheter op basis van MR beeldvorming in real-time) bereikt wordt. Invasieve bloeddrukmetingen in combinatie met een snelheidsgecodeerde CMR scantechniek voor de bepaling van bloedstroomsnelheden en -volumina in de aorta en longslagader werd als een nieuwe methode voorgesteld om perifere vaatweerstanden te berekenen.

Vervolgens wordt in hoofdstuk drie uitgelegd dat zelfs geringe links-rechts-shuntvolumina met MR stroomsnelheidsmetingen correct aangetoond kunnen worden (Qp : Qs < 1.5). Tevens werd de grootte van een atriaal septumdefect (ASD) voor de eerste keer middels een meetballon onder MR-doorlichting gemeten. Hiermee kan de grootte van een ASD ook bij kleine shuntvolumina goed bepaald worden. Tenslotte werd het defect met een kathetergebaseerd toestel interventioneel gesloten onder MR-doorlichting.

Concluderend hebben we aangetoond dat linker en rechter hartkatheterisaties onder MR-doorlichting mogelijk zijn en met name bij kinderen met aangeboren hartafwijkingen de potentie hebben om de gewone röntgendoorlichting te vervangen om de stralingsexpositie te voorkomen. Hemodynamische katheterisatiedata kan met anatomisch en functioneel CMR gecombineerd worden om de evaluatie van complex congenitaal hartlijden te verbeteren. Er zijn nu vele studies in dier en mens verricht die aantonen dat MR-doorlichting en hartkatheterisaties goed mogelijk zijn (proof-of-concept studies). De introductie van MR-doorlichting in de dagelijkse praktijk hangt echter sterk af van de ontwikkeling en de beschikbaarheid van MR-compatibel katheter- en voerdraadmateriaal. Ook het scannerdesign moet voor interventionele procedures verder verbeterd worden, zoals open MR-magneten, kortere magneten of grotere tunnelbuizen in de magneten. Op dit moment wordt CMR-doorlichting samen met interventies alleen toegepast bij kinderen met aangeboren hartaandoeningen en in de elektrofysiologie waar het om het tonen van afwijkende weefselstructuur en nauwkeurig afbeelden van complexe anatomische details gaat.

Met name bij de diagnostiek van patiënten met aangeboren hartafwijkingen is CMR niet meer weg te denken. Hier is de verbinding van anatomische beelden met hemodynamische data op basis van metingen van bloedstroomsnelheden en -volumina (flow) al langer dagelijkse praktijk. Bij volwassenen worden bloedstroomsnelheidsmetingen bijvoorbeeld bij de nauwkeurige bepaling van kleplekkages verricht. Als de snelheidsmetingen sneller en in real-time zouden kunnen gebeuren, zijn nog veel meer hemodynamische metingen mogelijk, bijvoorbeeld ook tijdens bepaalde manoeuvres zoals diepe inspiratie of tijdens verhoging van de hartslag. Het volgende hoofdstuk is een voorbeeld van hoe dit uitgevoerd zou kunnen worden, echter moeten de sequenties nog verbeterd worden: in hoofdstuk vier wordt een real-time CMR-methode voor de meting van bloedstroomsnelheden in verschillende slagaders gepresenteerd. ECG-triggering was 
niet nodig en de scantijden werden van twee minuten naar zes seconden verkort. In combinatie met het interactieve plannen van scans maakte de real-time meetmethode het mogelijk om al tijdens het scannen de scanvlakken snel aan te passen. De maximale snelheden correleerden in alle slagaders met de standaard techniek, bij de metingen van de volume flow was dit alleen in de allergrootste vaten het geval. De snelle evaluatie van flow tijdens fysiologische manoeuvres of toedienen van stressmedicatie is met real-time flow sequenties dus in principe mogelijk.

Het opsporen van regionale wandbewegingsstoornissen als uiting van myocardiaal zuurstoftekort tijdens dobutamine stress met MR real-time beeldvorming wordt in hoofdstuk vijf beschreven en vergeleken met een standaardtechniek. De sensitiviteit en de specificiteit van de twee technieken waren vergelijkbaar goed. De beeldkwaliteit van de real-time techniek was vergelijkbaar met de standaard techniek wat betreft de korte as opnames van het hart, maar inferieur voor de andere hartassen. Ondertussen zijn de standaardsequenties vervangen door balanced steady state free precession sequenties. Op dit moment zijn verschillende ontwikkelingen bij de real-time sequenties te zien die tot duidelijke verbetering van de beeldkwaliteit leiden.

Concluderend worden de real-time beeldvormingstechnieken nu overal in het begin van elk CMR-onderzoek bij de planning van de scans toegepast, maar nog niet als hoofdsequenties in verband met de minder goede beeldkwaliteit. Echter kunnen we ons de lange scantijden en ademcommando's bij de standard sequenties niet meer langer permitteren en real-time beeldvorming kan hierbij helpen. De beeldkwaliteit kan en moet nog beter en er wordt hier momenteel dan ook veel onderzoek naar gedaan. Verder moeten ook de uitwerkprogramma's aangepast worden omdat ze op dit moment de ECG-synchronisatie voor de berekening van onder andere de volumina nog nodig hebben. Röntgendoorlichting is de standaard techniek voor coronair- en klepinterventies, maar electrofysiologisch onderzoek met interventies onder MR-doorlichting is al om de hoek.

Het tweede deel van dit proefschrift bevat hoofdstukken over de waarde van CMR bij de diagnostiek van ischemische hartziekten (zuurstoftekort en infarcten). Op dit moment gaat het bij patiënten met een acuut hartinfarct erom met een dotterprocedure zo snel mogelijk de afgesloten kransslagader te heropenen. Dit lukt binnen Nederland al goed en door het verder verkorten van de tijd tussen begin van het infarct en het dotteren is waarschijnlijk geen verder overlevingsvoordeel te behalen. Het is dus belangrijk om nieuwe strategieën te bedenken die de gevolgen van een hartinfarct beperken en de beeldvorming van infarcten met CMR is hierbij heel belangrijk. Dus wordt in het tweede deel van het proefschrift de detectie en groottemeting van hartinfarcten met CMR beschreven (hoofdstukken zes tot en met acht). Het begint met het opsporen en meten door middel van een necrose-specifiek contrastmiddel in een diermodel van normale en gehypertrofieerde harten. Gehypertrofieerde linker ventrikels toonden duidelijk grotere infarcten dan normale harten. Als tijdens het infarct een kaliumkanaalopener acuut toegediend werd, waren de infarcten in gehypertrofieerde harten weer 
van vergelijkbare grootte als in de normale harten. Chronische toediening over een lange periode vóór het infarct voorkwam het ontwikkelen van hypertrofie en de infarctgrootte was dan ook weer hetzelfde als in de controlegroep. De resultaten van de CMRmetingen waren hetzelfde als de histologische infarct- en massabepalingen. CMR zal dus een belangrijke rol bij de evaluatie van nieuwe behandelstrategieën voor patiënten met hartinfarct kunnen spelen. Ook hoofdstuk negen onderstreept de waarde van CMR bij de diagnostiek van hartinfarcten. CMR werd met echocardiografie vergeleken bij het opsporen van acute en oude infarcten. Het bleek dat echocardiografie $20 \%$ van de acute en $40 \%$ van de oude infarcten mist. De gemiste infarcten waren in vergelijking met de gedetecteerde infarcten meestal kleiner en betroffen niet de volledige dikte van de hartspier.

Verder is MR perfusie een belangrijke techniek om zuurstoftekort in de hartspier aan te tonen. Hoofdstuk tien beschrijft patiënten met pijn op de borst en zuurstoftekort in de hartspier bij inspanning die niet op vernauwingen van de kransslagaders berust. Waarschijnlijk berust het zuurstoftekort bij deze patiënten met het cardiale syndroom $\mathrm{X}$ op veranderingen van de haarvaatjes, de zogenoemde microvasculatuur, en de prognose van deze patiënten is niet beter dan van patiënten met belangrijke vernauwingen van de krasslagaders. Tot nu toe is het niet mogelijk om de microvasculatuur direct in beeld te brengen. We hebben met een nieuwe methode de veranderingen van de microvasculatuur onder de tong met behulp van een camera bij deze patiënten bestudeerd en aansluitend de bloeddoorstroming van de hartspier met stress MR gemeten. Patiënten met syndroom $X$ hadden een microvasculaire dysfunctie bij de camerametingen en daarnaast een duidelijk mindere toename van de bloeddoorstroming van het binnenste gedeelte van de hartspier bij stress.

Het derde deel van dit proefschrift beschrijft de waarde van CMR in niet op zuurstoftekort berustende hartziekten. Hierbij gaat het om drie verschillende unieke aspecten van beeldvorming met CMR: het opsporen van littekenvorming in het hart, functiebepaling van de rechter hartkamer en het in kleine groepen van patiënten (zoals bij patiënten met spierziekten) nauwkeurig opsporen van hartafwijkingen. Hoofdstuk elf laat de resultaten zien van onderzoek naar focale (spier-vervangende) en interstitiële (reactieve) bindweefsel- of littekenvorming in de hartspier van patiënten met gedilateerde cardiomyopathie. Focale bindweefselvorming werd met CMR bepaald en interstitiële in biopten na hartbiopsie. Patiënten met focale bindweefselvorming hebben een slechtere prognose dan patiënten zonder bindweefselvorming. Een van onze hypotheses was dat focale bindweefselvorming pas bij een grote hoeveelheid aan interstitiële bindweefselvorming op te sporen is. Dit was niet het geval. Er was geen correlatie tussen focale en interstitiële bindweefselvorming. De aanwezigheid van focale bindweefselvorming was echter wel gerelateerd aan ontstekingscelactiviteit.

Alle beeldvormingsmodaliteiten met uitzondering van MR struikelen over de nauwkeurige meting van de functie en grootte van de rechter hartkamer. De rechter kamerfunctie is sterk bepalend voor het inspanningsvermogen en de prognose van patiënten 
met hartfalen. De rechter ventrikel is duidelijk minder vaak onderzocht dan de linker ventrikel in de wetenschappelijke literatuur en de dagelijkse praktijk en wordt dan ook wel 'de vergeten hartkamer' genoemd. De grootte en functie van de rechter hartkamer gemeten met CMR bij patiënten met gedilateerde cardiomyopathie worden in hoofdstuk twaalf vergeleken met de grootte en functie bij patiënten met hartinfarcten. De rechter ventrikel functie was beïnvloed door het knijp- en ontspanningsvermogen van de linker ventrikel en door de bloeddruk in de longslagader. De rechter ventrikel functie was bij de patiënten met gedilateerde cardiomyopathie duidelijk slechter dan bij de hartinfarctpatiënten.

Door de nauwkeurigheid van CMR bij de volume- en ejectiefractie bepalingen van rechter en linker kamer, spiermassaberekening, opsporen van littekens en infarcten is het aangetoond dat hartafwijkingen met CMR goed aangetoond kunnen worden in kleine groepen van patiënten. Hoofdstuk dertien is een beschrijving van de CMR bevindingen bij patiënten met de spierziekte myotone dystrofie type I. Patiënten met deze zeldzame aandoening hebben vaker geleidingsstoornissen en soms ook structurele veranderingen van het hart met hartspierzwakte. Met CMR waren bij $44 \%$ van de patienten afwijkingen aan het hart te zien. Een normaal ECG sluit hartafwijkingen bij patiënten met myotone dystrofie niet uit.

In de discussie aan het eind van het proefschrift (hoofdstuk veertien) worden de hoofdboodschappen samengevat en in de context van de wetenschappelijke literatuur besproken. CMR heeft zich tot een in de dagelijkse praktijk met uitstekende diagnostische waarde toegepaste techniek voor beeldvorming van hart en vaten ontwikkeld. Vijf belangrijke punten worden geïdentificeerd die voor de toekomstige ontwikkeling van CMR bepalend zijn.

1) Een CMR-onderzoek moet in de toekomst nog sneller worden en de beelden moeten vaker met de real-time techniek gemaakt worden. Steeds kortere onderzoekstijden zijn voor patiënten minder belastend.

2) De MR apparaten moeten kleiner worden: kortere magneten, grotere tunnelbuizen in de magneten en open MR-magneten. Ondanks dat CMR een veilig onderzoek is zijn vele patiënten nog bang voor het grote apparaat.

3) Op het gebied van CMR moet men meer van anderen gaan leren. Het is verder belangrijk om nieuwe ontwikkelingen op het gebied van scansequenties sneller met elkaar te delen, scanprotocollen nog meer te standaardiseren en het scannen makkelijker te maken.

4) Reeds beschikbare technieken moeten verbeterd en ook beter toegepast worden. 3D beeldvorming bestaat al en wordt nog niet standaard toegepast. Methodes om de diastolische functie van de hartkamers te meten, klepgebreken op te sporen of de kransslagaders beter in beeld te brengen bestaan ook al, maar eisen op dit moment nog te veel tijd met betrekking tot het maken en analyseren van de beelden.

5) Het is belangrijk om nog beter te onderbouwen dat CMR cruciaal is voor de diagnosestelling, therapie en prognose van patiënten met hartaandoeningen. Beeldvor- 
ming speelt een belangrijke rol in het diagnostische en therapeutische proces en wordt steeds vaker toegepast in een omgeving met gelimiteerde financiële resources. Meer onderzoek is vereist met betrekking tot kosteneffectiviteit, diagnostische waarde en invloed op prognose en kwaliteit van leven van patiënten met hartziekten.

Op het gebied van hartbeeldvorming met magnetische resonantie is de afgelopen twee decennia al veel bereikt. Er zijn vijf grote uitdagingen voor de toekomst en nog vele malen meer kansen. De toekomst is veelbelovend. 


\section{Valorization}

Since the first publication of a cross sectional magnetic resonance image of the human chest in 1977, cardiovascular magnetic resonance imaging (CMR) has emerged as a reliable imaging technique and is currently performed on a routine basis for many patients with heart disease. The burden of cardiac disease in the Netherlands in 2012 was 20555 cardiac deaths and 202945 hospital admissions. More than 1 million patients suffer from cardiovascular disease. CMR is an important tool to detect cardiac disease and it is performed to support the treating physicians in finding the correct diagnosis and evaluate therapy. The research presented in this thesis is therefore of value for patients with heart disease and their caring physicians. Since the thesis describes many different aspects of magnetic resonance imaging of cardiac function, a point-by-point evaluation of valorization would result in a repetition of the discussion-chapter. Thus, to underscore the importance of research for daily practice, real-time imaging is chosen from this thesis as an example of a successful implementation of research into practice. With real-time imaging, patient comfort is improved since it is fast and does not require repeated breath-holds and ECG-gating. Images can already be displayed during the process of scanning. The price of improved patient comfort usually is less image quality. However, it could be shown that real-time imaging offers sufficient image quality for several clinical applications. Thus, it has been introduced into daily practice: Real-time imaging is performed in the beginning of every CMR exam for the purpose of interactive scan planning. It saves time and makes the process of planning the correct heart axes easier. Real-time imaging sequences are also currently employed to replace standard breath-hold, ECG-triggered cine sequences in case of suboptimal image quality due to an irregular heart rhythm or the patient is not able to hold his breath. 



\section{Dankwoord}



Dit proefschrift bevat al veel inhoud. Ik zal zeker ook de dankbetuiging kort en krachtig houden: mijn allereerste dank gaat uit naar mijn patiënten. Zij zijn met hun ervaringen een bron aan inspiratie. Mijn dank gaat verder uit naar de promotoren professor H.J.G.M. Crijns en professor J.E. Wildberger voor hun inspirerende rol, de begeleiding en het mogelijk maken van de promotie. De leden van de beoordelingscommissie wil ik graag danken voor hun geduld en inspanningen. Ik mocht genieten van ontmoetingen met vele mensen die open en steunend voor mij waren. Mijn dank is groot! Afdelingshoofden, opleiders, onderzoekers, collegae, assistenten, laboranten, verpleegkundigen, secretaressen en medewerkers uit Heidelberg (universiteit en German Cancer Research Center), Ulm en Tübingen (universitaire ziekenhuizen), Berlijn (German Heart Institute met Charité Campus Virchow), San Francisco (University of California) en Maastricht (iedereen!) zijn van harte bedankt. Dank aan de beeldvormingsploegen van cardiologie en radiologie.

Tot slot: ondertussen ben ik al meer dan 15 jaar bezig met beeldvorming van het hart met behulp van magnetische resonantie. De European Association of Cardiovascular Imaging heeft mij vervolgens erkend als grootvader op het gebied van MR: het lijkt me dan ook logisch om in de introductie een kort overzicht van de geschiedenis van beeldvorming van het hart met MR te tonen. Mijn droombaan was het werken in de landbouw. Helaas had ik geen eigen grond en ben vervolgens alleen maar cardioloog geworden. Dit bleek een verstandige keuze omdat zowel voor de landbouw als ook voor de cardiologie een grote portie gezond boerenverstand vereist is. In principe komt de cardiologie op de volgende eenvoudige formules neer:

"Cardiologie $=$ vullingsstatus + hemodynamiek + ischemie + ritme + inflammatie + psychologie"

"Niet-invasieve cardiologie = patiëntenzorg + beeldvorming"

"Invasieve cardiologie = patiëntenzorg + ballon opblazen of lijntjes trekken tijdens beeldvorming"

Tot daadwerkelijk slot: dank aan Maastricht en de rest van Nederland. Ik realiseer me dat de volgende zinnen met uiterste voorzichtigheid te genieten zijn. Het gaat om Nederland versus Duitsland (ook al gaat het niet over voetbal). Ik ben ervan overtuigd dat de Nederlanders een "California"-kant hebben: cool, laagdrempelig en positief denkend. Omdat het mij nog steeds een beetje ontbreekt aan coolness en aan dingen niet te serieus te nemen, leer ik iedere dag van jullie.

Tot echt slot: werk is werk en privé is privé. Liefste Carmen, je bent mijn allergrootste schat. Louise, Sophie en Elias, ik ben zoo00000000000000 trots op jullie. Ouders, familie: dank.

Tot concluderend slot: onze baan, de patiënten, het onderzoek, het is niet altijd allemaal makkelijk. En als het wel altijd makkelijk was, dan was het niets voor cardiologen! 

Curriculum vitae 

Simon Michael Schalla was born in 1967 in Kiel, Germany. After graduation from Gymnasium Altenholz-Stift in 1986, he served his period of social work as a paramedic at the ambulance service of the Red Cross Kiel until 1988. He subsequently studied medicine at the University of Heidelberg Medical School (1988-1995) and Harvard Medical School, Boston, USA (1994). From 1992 to 1993 he had a stipend to follow the research program on experimental radiotherapy with fast neutrons at the German Cancer Research Center in Heidelberg (Prof. dr. W.J. Lorenz, Prof. dr. G. van Kaick). He obtained his medical degree in 1995. Postgraduate medical training followed from 1995 to 1996 at the University Hospital UIm (Prof. dr. G. Adler), from January to September 1997 at the University Hospital Tübingen (Prof. dr. M. Gregor) and from October 1997 to November 2003 at the German Heart Institute Berlin and Charite, Humboldt University, Berlin (Prof. dr. E. Fleck, Prof. dr. E. Nagel). In 2001 he registered as internist in Germany. From October 2001 to April 2003 he was a fellow of the German Cardiac Society at the University of California San Francisco, Cardiac MR imaging (Prof. dr. C.B. Higgins, Prof. dr. M. Saeed). Since December 2003 he works at the Maastricht University Medical Center, Department of Cardiology (Prof. dr. H.J.G.M. Crijns), and since 2014 also at the Department of Radiology (Prof. dr. J. Wildberger). He registered as internist and cardiologist in the Netherlands. He is involved in clinical reading, teaching and research in cardiovascular MR imaging since 1997 (German Heart Institute Berlin, University of California San Francisco, Maastricht University Medical Center). 

List of publications 

Schalla S, Jaarsma C, Bekkers SC, Waltenberger J, Dennert R, Crijns HJ, Wildberger J, Heymans S, Brunner-La Rocca HP. Non-invasive Assessment of Right Ventricular Dysfunction in Dilated Cardiomyopathy and Ischemic Heart Disease. Neth Heart J. 2015 Apr;23(4):232-240.

Jaarsma C, Smulders MW, Nelemans PJ, Bekkers SC, Bucerius J, Leiner T, Crijns HJ, Wildberger J, Schalla S. Excellent prognosis after a negative test result -A meta-analysis of the prognostic value of currently performed non-invasive cardiac imaging modalities in patients with suspected or known coronary artery disease. Submitted.

Jaarsma C, van Haare J, Vink H, Bekkers SC, van Rooijen BD, Backes WH, Wildberger J, Crijns HJ, van Teeffelen J, Schalla S. Non-invasive assessment of microvascular dysfunction in patients with cardiac syndrome X. Submitted.

Hazebroek MR, Kemna MJ, Schalla S, Sanders-van Wijk S, Gerretsen SC, Dennert R, Kuznetsova T, Staessen JA, Brunner-La Rocca HP, van Paassen P, Cohen Tervaert JW, Heymans $\mathrm{S}$. Prevalence and prognostic relevance of cardiac involvement in ANCAassociated vasculitis. Submitted.

El Aidi H, Adams A, Moons KG, Den Ruijter HM, Mali WP, Doevendans PA, Nagel E, Schalla S, Bots ML, Leiner T. Cardiac Magnetic Resonance Imaging Findings and the Risk of Cardiovascular Events in Patients With Recent Myocardial Infarction or Suspected or Known Coronary Artery Disease. J Am Coll Cardiol. 2014 Mar 25;63(11):1031-1045.

Smulders MW, Kietselaer BL, Das M, Wildberger JE, Crijns HJ, Veenstra LF, Brunner-La Rocca HP, van Dieijen-Visser MP, Mingels AM, Dagnelie PC, Post MJ, Gorgels AP, van Asselt AD, Vogel G, Schalla S, Kim RJ, Bekkers SC. The role of cardiovascular magnetic resonance imaging and computed tomography angiography in suspected non-STelevation myocardial infarction patients: design and rationale of the CARdiovascular Magnetic rEsoNance imaging and computed Tomography Angiography (CARMENTA) trial. Am Heart J. 2013 Dec;166(6):968-75.

Jaarsma C, Bekkers SC, Haidari Z, Smulders MW, Nelemans PJ, Gorgels AP, Crijns HJ, Wildberger JE, Schalla S. Comparison of different electrocardiographic scoring systems for detection of any previous myocardial infarction as assessed with cardiovascular magnetic resonance imaging. Am J Cardiol. 2013 Oct 15;112(8):1069-74.

Jaarsma C, Nagel E, Schalla S. A Critical Review of Different Imaging Methods for the Assessment of Myocardial Ischemia. Curr Cardiovasc Imaging Rep. 2013 Apr 1;6(2):117127. 
Jaarsma C, Schalla S, Cheriex EC, Smulders MW, van Dongen I, Nelemans PJ, Gorgels AP, Wildberger JE, Crijns HJ, Bekkers SC. Incremental value of cardiovascular magnetic resonance over echocardiography in the detection of acute and chronic myocardial infarction. J Cardiovasc Magn Reson. 2013 Jan 16;15:5.

Hermans MC, Faber CG, Bekkers SC, de Die-Smulders CE, Gerrits MM, Merkies IS, Snoep G, Pinto YM, Schalla S. Structural and functional cardiac changes in myotonic dystrophy type 1: a cardiovascular magnetic resonance study. J Cardiovasc Magn Reson. 2012 Jul 24;14:48.

van der Weg K, Bekkers SC, Winkens B, Lemmert ME, Schalla S, Crijns HJ, Waltenberger J, Gorgels AP. Evaluation of the electrocardiogram in identifying and quantifying lateral involvement in nonanterior wall infarction using cardiovascular magnetic resonance imaging. J Electrocardiol. 2012 Sep;45(5):478-84.

Jaarsma C, Leiner T, Bekkers SC, Crijns HJ, Wildberger JE, Nagel E, Nelemans PJ, Schalla $\mathrm{S}$. Diagnostic performance of noninvasive myocardial perfusion imaging using singlephoton emission computed tomography, cardiac magnetic resonance, and positron emission tomography imaging for the detection of obstructive coronary artery disease: a meta-analysis. J Am Coll Cardiol. 2012 May 8;59(19):1719-28.

Bekkers SC, Lemmert ME, Passos VL, Mihl C, Schalla S, Wildberger JE, Waltenberger J, Gorgels AP. The relationships between cardiovascular magnetic resonance imaging variables of acute myocardial infarction and both left ventricular dysfunction and immediate postreperfusion ST segment recovery. J Electrocardiol. 2011 SepOct;44(5):561-7

van Rijsingen IA, Bekkers SC, Schalla S, Hermans-van Ast JF, Snoep G, Alzand BS, Arens $\mathrm{YH}$, van den Wijngaard A, Crijns HJ, Pinto YM. Exercise related ventricular arrhythmias are related to cardiac fibrosis in hypertrophic cardiomyopathy mutation carriers. Neth Heart J. 2011 Apr;19(4):168-174.

Gerretsen SC, Kooi ME, Kessels AG, Schalla S, Katoh M, van der Geest RJ, Manning WJ, Waltenberger J, van Engelshoven JM, Botnar RM, Leiner T. Visualization of coronary wall atherosclerosis in asymptomatic subjects and patients with coronary artery disease using magnetic resonance imaging. PLoS One. 2010 Sep 29;5(9). pii: e12998

Schalla S, Walenkamp G, Opdenakker L, Maessen J. Never-changing mediastinal nodules. Eur J Cardiothorac Surg. 2011 May;39(5):796. 
Verjans J, Wolters S, Laufer W, Schellings M, Lax M, Lovhaug D, Boersma H, Kemerink G, Schalla S, Gordon P, Teule J, Narula J, Hofstra L. Early molecular imaging of interstitial changes in patients after myocardial infarction: comparison with delayed contrastenhanced magnetic resonance imaging. J Nucl Cardiol. 2010 Dec;17(6):1065-72.

Jaarsma C, Nijs J, Gommers S, Bekkers SC, Schalla S. Images in cardiovascular medicine: Retrograde flow in the descending thoracic aorta: magnetic resonance imaging of an apicoaortic conduit. Circulation. 2010 Jul 13;122(2):e12

Dennert R, Velthuis S, Westermann D, Donker D, Schalla S, van Suylen RJ, Heymans S. Parvovirus-B19-associated fulminant myocarditis successfully treated with immunosuppressive and antiviral therapy. Antivir Ther. 2010;15(4):681-5.

Bekkers SC, Smulders MW, Passos VL, Leiner T, Waltenberger J, Gorgels AP, Schalla S. Clinical implications of microvascular obstruction and intramyocardial haemorrhage in acute myocardial infarction using cardiovascular magnetic resonance imaging. Eur Radiol. 2010 Nov;20(11):2572-8.

Dennert R, Velthuis S, Schalla S, Eurlings L, van Suylen RJ, van Paassen P, Tervaert JW, Wolffs P, Goossens VJ, Bruggeman C, Waltenberger J, Crijns HJ, Heymans S. Intravenous immunoglobulin therapy for patients with idiopathic cardiomyopathy and endomyocardial biopsy-proven high PVB19 viral load. Antivir Ther. 2010;15(2):193-201.

Schalla S, Bekkers SC, Dennert R, van Suylen RJ, Waltenberger J, Leiner T, Wildberger J, Crijns HJ, Heymans S. Replacement and reactive myocardial fibrosis in idiopathic dilated cardiomyopathy: comparison of magnetic resonance imaging with right ventricular biopsy. Eur J Heart Fail. 2010 Mar;12(3):227-31.

Dennert RM, van Paassen P, Schalla S, Kuznetsova T, Alzand BS, Staessen JA, Velthuis S, Crijns HJ, Tervaert JW, Heymans S. Cardiac involvement in Churg-Strauss syndrome. Arthritis Rheum. 2010 Feb;62(2):627-34.

van Rijsingen IA, Hermans-van Ast JF, Arens YH, Schalla S, de Die-Smulders CE, van den Wijngaard A, Pinto YM. Hypertrophic cardiomyopathy family with double-heterozygous mutations; does disease severity suggest doubleheterozygosity? Neth Heart J. 2009 Dec;17(12):458-63.

Dennert R, Schalla S, Heymans S. Cardiomyocyte disintegration during Anderson-Fabry's disease. Eur Heart J. 2010 Apr;31(8):917. 
Delnoij T, van Suylen RJ, Cleutjens JP, Schalla S, Bekkers SC. In vivo histology by cardiovascular magnetic resonance imaging. Eur Heart J. 2009 Oct;30(20):2492.

Bekkers SC, Backes WH, Kim RJ, Snoep G, Gorgels AP, Passos VL, Waltenberger J, Crijns $\mathrm{HJ}$, Schalla S. Detection and characteristics of microvascular obstruction in reperfused acute myocardial infarction using an optimized protocol for contrast-enhanced cardiovascular magnetic resonance imaging. Eur Radiol. 2009 Dec;19(12):2904-12.

Dennert R, Schalla S, Suylen RJ, Eurlings L, Heymans S. Giant cell myocarditis triggered by a parvovirus B19 infection. Int J Cardiol. 2009 May 1;134(1):115-6.

Bekkers SC, Lemmert ME, Schalla S, Snoep R, Crijns HJ, Gorgels AP. Multivessel myocardial infarction. Neth Heart J. 2007 Dec;15(12):424-5

Gerretsen SC, Kooi ME, Schalla S, Delhaas T, Snoep G, Van Engelshoven JM, Leiner T. Magnetic resonance imaging of the coronary arteries. Cardiovasc J Afr. 2007 JulAug;18(4):248-59.

Herskind C, Schalla S, Hahn EW, Höver KH, Wenz F. Influence of different dose rates on cell recovery and RBE at different spatial positions during protracted conformal radiotherapy. Radiat Prot Dosimetry. 2006;122(1-4):498-505.

Meijers BK, Schalla S, Eerens F, Van Suylen RJ, Broers B, Cheriex EM, Smedema JP. Protein-losing enteropathy in association with constrictive pericarditis. Int J Cardiovasc Imaging. 2006 Jun-Aug;22(3-4):389-92.

Schalla S, Bär F, Mochtar B, Snoep G, Bekkers SC. Left ventricular pseudoaneurysm. Eur Heart J. 2006 Apr;27(7):807.

Schalla S, Higgins CB, Saeed M. Long-term oral treatment with nicorandil prevents the progression of left ventricular hypertrophy and preserves viability. J Cardiovasc Pharmacol. 2005 Apr;45(4):333-40.

Schalla S, Saeed M, Higgins CB, Weber O, Martin A, Moore P. Balloon sizing and transcatheter closure of acute atrial septal defects guided by magnetic resonance fluoroscopy: assessment and validation in a large animal model. J Magn Reson Imaging. 2005 Mar;21(3):204-11. 
Paetsch I, Föll D, Langreck H, Herkommer B, Klein C, Schalla S, Fleck E, Nagel E. Myocardial perfusion imaging using OMNISCAN: a dose finding study for visual assessment of stress-induced regional perfusion abnormalities. J Cardiovasc Magn Reson. 2004;6(4):803-9.

Schalla S, Higgins CB, Chujo M, Saeed M. Effect of potassium-channel opener therapy on reperfused infarction in hypertrophied hearts: demonstration of preconditioning by using functional and contrast-enhanced magnetic resonance imaging. J Cardiovasc Pharmacol Ther. 2004 Sep;9(3):193-202.

Saeed M, Lee R, Martin A, Weber O, Krombach GA, Schalla S, Lee M, Saloner D, Higgins $\mathrm{CB}$. Transendocardial delivery of extracellular myocardial markers by using combination X-ray/MR fluoroscopic guidance. Radiology. 2004 Jun;231(3):689-96.

Schalla S, Wendland MF, Higgins CB, Ebert W, Saeed M. Accentuation of high susceptibility of hypertrophied myocardium to ischemia: complementary assessment of Gadophrin-enhancement and left ventricular function with MRI. Magn Reson Med. 2004 Mar;51(3):552-8.

Weber OM, Schalla S, Martin AJ, Saeed M, Lee R, Shunk KA, Moore P, Higgins CB. Interventional cardiac magnetic resonance imaging. Semin Roentgenol. 2003 Oct;38(4):3527.

Schalla S, Saeed M, Higgins CB, Martin A, Weber O, Moore P. Magnetic resonance-guided cardiac catheterization in a swine model of atrial septal defect. Circulation. 2003 Oct 14;108(15):1865-70.

Klein C, Schalla S, Schnackenburg B, Bornstedt A, Hoffmann V, Fleck E, Nagel E. Improvement of image quality of non-invasive coronary artery imaging with magnetic resonance by the use of the intravascular contrast agent Clariscan (NC100150 injection) in patients with coronary artery disease. J Magn Reson Imaging. 2003 Jun;17(6):656-62.

Nagel E, Thouet T, Klein C, Schalla S, Bornstedt A, Schnackenburg B, Hug J, Wellnhofer E, Fleck $\mathrm{E}$. Noninvasive determination of coronary blood flow velocity with cardiovascular magnetic resonance in patients after stent deployment. Circulation. 2003 Apr 8;107(13):1738-43.

Schalla S, Higgins CB, Saeed M. Contrast agents for cardiovascular magnetic resonance imaging. Current status and future directions. Drugs R D. 2002;3(5):285-302. 
Schalla S, Klein C, Paetsch I, Lehmkuhl H, Bornstedt A, Schnackenburg B, Fleck E, Nagel $E$. Real-time MR image acquisition during high-dose dobutamine hydrochloride stress for detecting left ventricular wall-motion abnormalities in patients with coronary arterial disease. Radiology. 2002 Sep;224(3):845-51.

Nagel E, Schneider U, Schalla S, Ibrahim T, Schnackenburg B, Bornstedt A, Klein C, Lehmkuhl HB, Fleck E. Magnetic resonance real-time imaging for the evaluation of left ventricular function. J Cardiovasc Magn Reson. 2000;2(1):7-14.

Klein C, Schalla S, Schnackenburg B, Bornstedt A, Fleck E, Nagel E. Magnetic resonance flow measurements in real-time: comparison with a standard gradient-echo technique. J Magn Reson Imaging. 2001 Sep;14(3):306-10.

Bornstedt A, Nagel E, Schalla S, Schnackenburg B, Klein C, Fleck E. Multi-slice dynamic imaging: complete functional cardiac MR examination within 15 seconds. J Magn Reson Imaging. 2001 Sep;14(3):300-5.

Klein C, Nagel E, Schnackenburg B, Bornstedt A, Schalla S, Hoffmann V, Lehning A, Fleck E. The intravascular contrast agent Clariscan (NC 100150 injection) for 3D MR coronary angiography in patients with coronary artery disease. MAGMA. 2000 Nov;11(1-2):65-7.

Schalla S, Nagel E, Lehmkuhl H, Klein C, Bornstedt A, Schnackenburg B, Schneider U, Fleck $\mathrm{E}$. Comparison of magnetic resonance real-time imaging of left ventricular function with conventional magnetic resonance imaging and echocardiography. Am J Cardiol. 2001 Jan 1;87(1):95-9.

Nagel E, Hug J, Bünger S, Bornstedt A, Schnackenburg B, Wellnhofer E, Klein C, Thouet T, Schalla S, Fleck E. Coronary flow measurements for evaluation of patients after stent implantation. MAGMA. 1998 Sep;6(2-3):184-5.

Schalla S, Herskind C, Höver KH, Lorenz WJ, Hahn EW. Changes in RBE of 14-MeV $(d+T)$ neutrons for $V 79$ cells irradiated in air and in a phantom: is RBE enhanced near the surface? Strahlenther Onkol. 1998 Apr;174(4):204-11. 


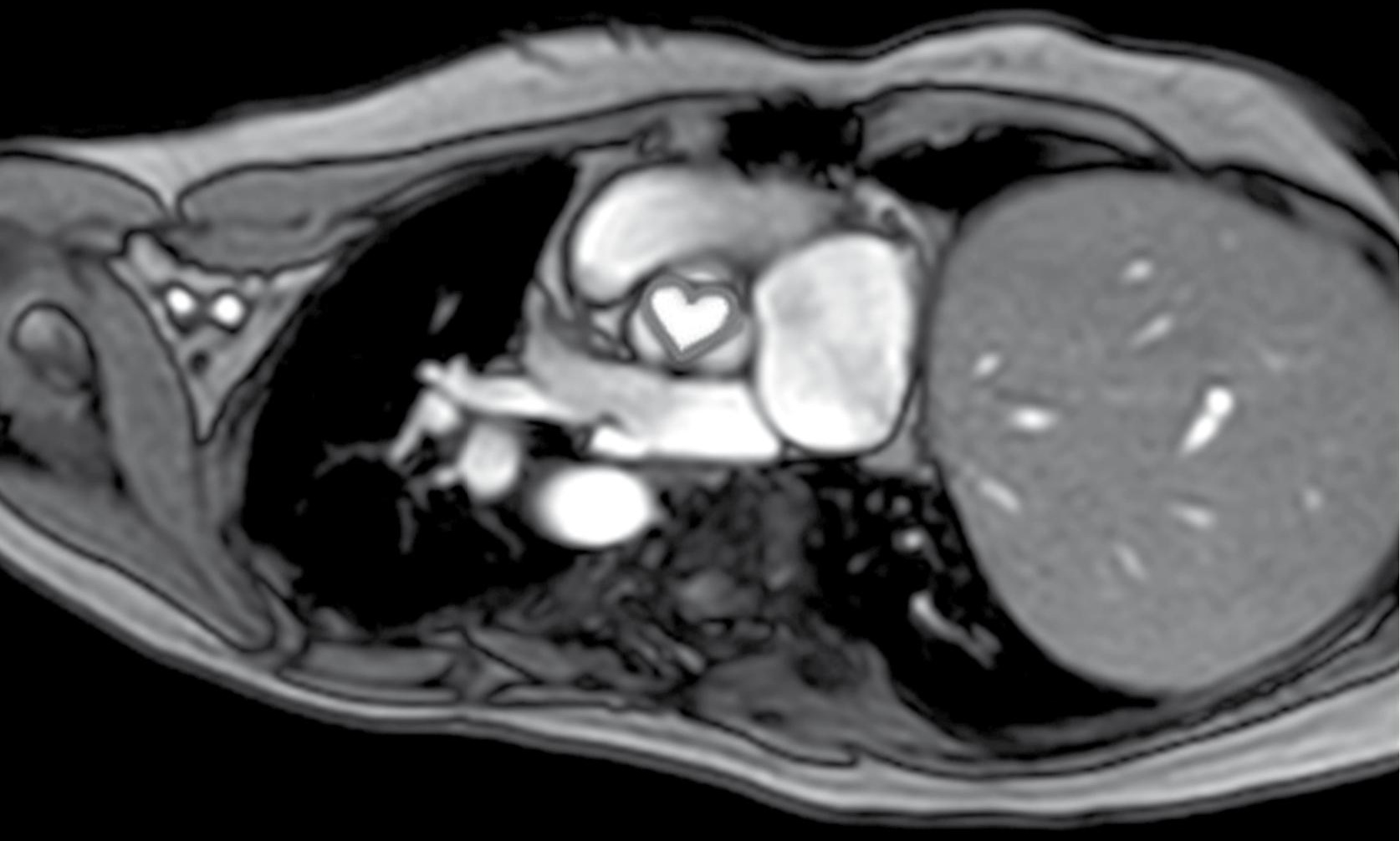

